\title{
NATIONAL WATER QUALITY LABORATORY 1994 SERVICES CATALOG
}

By Patricia J. Timme

U.S. GEOLOGICAL SURVEY

Open-File Report 94-304 


\section{U.S. DEPARTMENT OF THE INTERIOR \\ BRUCE BABBITT, Secretary \\ U.S. GEOLOGICAL SURVEY \\ Gordon P. Eaton, Director}

For additional information write to:

Chief, National Water Quality Laboratory

U.S. Geological Survey

Box 25046, Mail Stop 407

Federal Center

Denver, CO 80225
Copies of this report can be purchased from:

U.S. Geological Survey

Open-File Reports--ESIC

Box 25425

Federal Center

Denver, CO 80225-0425 


\section{CONTENTS}

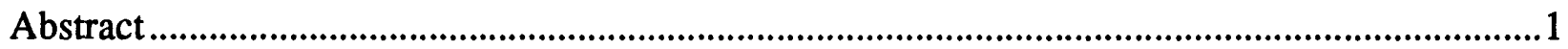

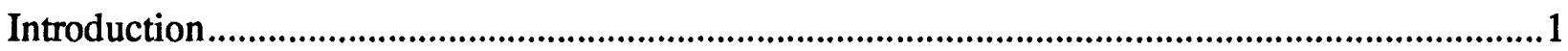

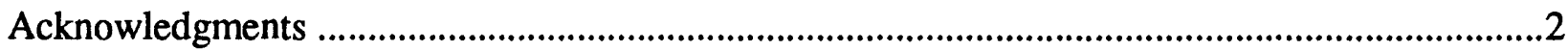

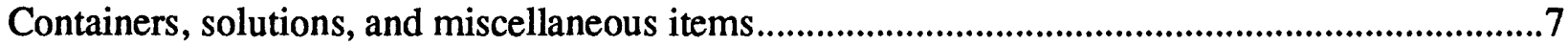

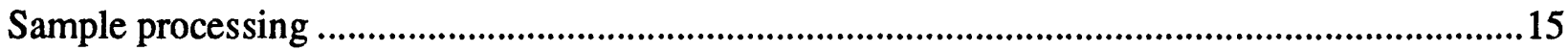

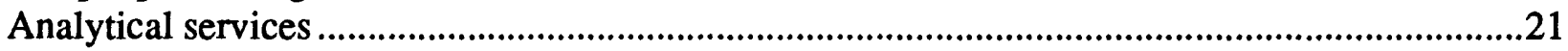

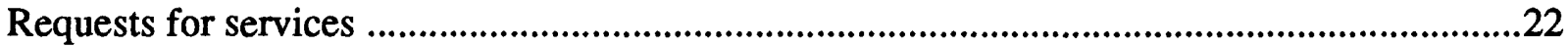

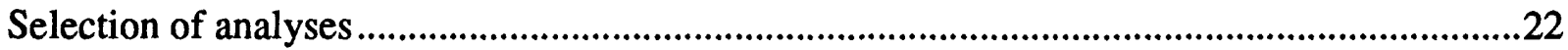

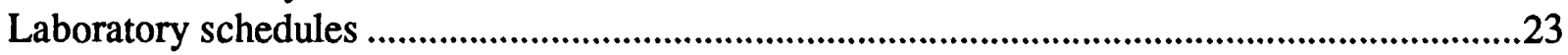

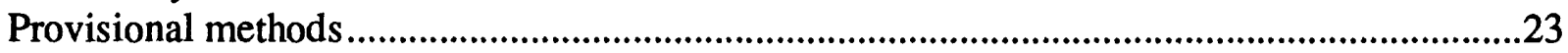

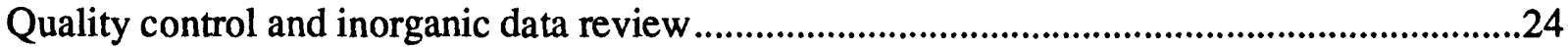

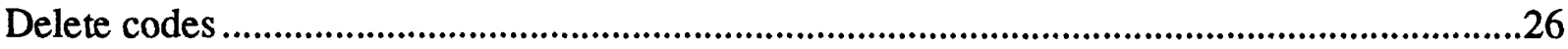

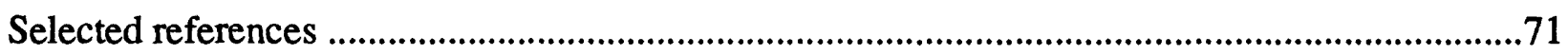

Appendix A--Description of schedules, parameters, and network program....................................73

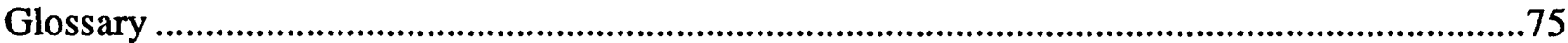

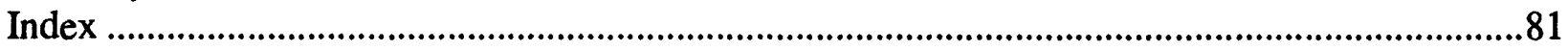

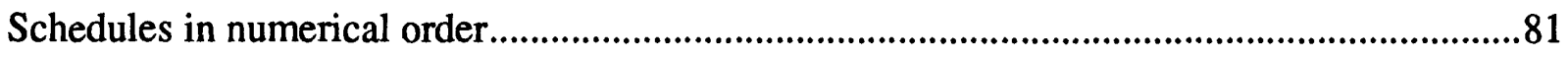

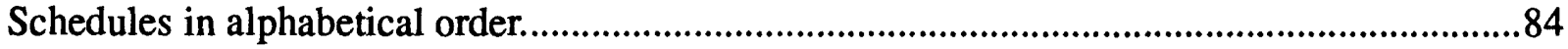

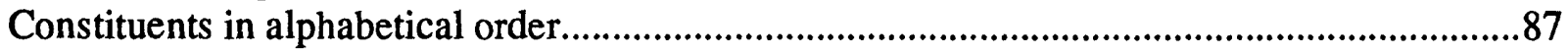

\section{TABLES}

Table 1. National Water Quality Laboratory contacts .....................................................................

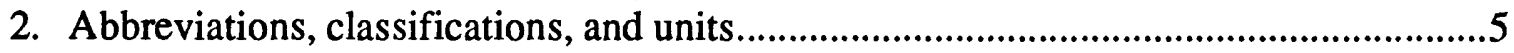

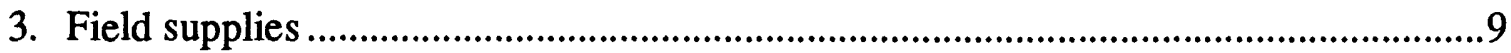

4. Sample designations, containers, and treatments.............................................................17

5. Biological determinations listed by laboratory schedule …………….............................27

6. Inorganic determinations for sediment listed alphabetically ...........................................29

7. Inorganic determinations for water listed alphabetically .................................................31

8. Inorganic determinations for sediment listed by laboratory schedule ............................37

9. Inorganic determinations for water listed by selected laboratory schedule ....................39

10. Inorganic determinations for tissue listed by laboratory schedule .................................41

11. Gross organic determinations listed alphabetically ......................................................43

12. Organic determinations for sediment listed by laboratory schedule.............................45

13. Organic determinations for water listed by laboratory schedule ...............................49

14. Organic determinations for tissue listed by laboratory schedule ..................................63

15. Radiochemical determinations listed by laboratory code and schedule ........................65

16. Stable isotope ratios listed by laboratory code and schedule.........................................69 


\section{CONVERSION FACTORS}

\begin{tabular}{lll}
\multicolumn{1}{c}{ Multiply } & By & To obtain \\
micrometer $(\mu \mathrm{m})$ & $3.94 \times 10^{-5}$ & inch \\
millimeter $(\mathrm{mm})$ & 0.03937 & inch \\
liter $(\mathrm{L})$ & 0.265 & gallon \\
microliter $(\mu \mathrm{L})$ & $2.64 \times 10^{-7}$ & gallon \\
milliliter $(\mathrm{mL})$ & $2.64 \times 10^{-4}$ & gallon \\
gram $(\mathrm{g})$ & 0.03527 & ounce, avoirdupois \\
milligram $(\mathrm{mg})$ & $3.53 \times 10^{-5}$ & ounce
\end{tabular}

Temperature in degrees Celsius $\left({ }^{\circ} \mathrm{C}\right)$ can be converted to degrees Fahrenheit $\left({ }^{\circ} \mathrm{F}\right)$ by using the following equation:

$$
{ }^{\circ} \mathrm{F}=9 / 5\left({ }^{\circ} \mathrm{C}\right)+32
$$




\begin{abstract}
This Services Catalog is intended for the internal use of U.S. Geological Survey personnel and contains information about field supplies and analytical services available from the National Water Quality Laboratory to Survey members of the Water Resources Division. To assist personnel in the selection of analytical services, this catalog lists sample volume, applicable concentration range, detection level, precision of analysis, and preservation requirements for samples.
\end{abstract}

\title{
INTRODUCTION
}

Three Water Resources DivisionTechnical Memoranda, 92.35--Use of Laboratories, 92.36--Use of Laboratories by National Water Quality Programs, and 92.37--Laboratory Methods, stated that all Water Resources Division (WRD) programs having national impact would use the services provided by the National Water Quality Laboratory (NWQL), whether in its own Laboratory, through contracted services, or by interagency agreements. Thus, all Districts and National Water-Quality Assessment (NAWQA) study units need to be familiar with the contents of this catalog.

This catalog is distributed annually but is continually updated to reflect new parameters, schedules, and analytical details. New methods of chemical analysis are constantly being researched, approved, and added at the NWQL. Parameters are deleted when they become obsolete. District owners of schedules might change their schedule contents, leaving users from other Districts with unwanted analytical work. To keep informed of these changes, readers of the catalog are urged to use a program on the NWQL partition of DCOLKA--Schedules, Parameters and Network (SPN). SPN contains the latest version of the information shown in this catalog, as well as historical information from the two previous fiscal years. SPN is a menu-driven search and display program, showing information about laboratory codes and schedule contents. However, no textual information is available in SPN.

The Services Catalog consists of three sections: the first section lists containers, solutions, and other materials that are available through the NWQL; the second section describes the field processing of samples submitted for analysis; and the third section includes available analytical services. In addition, a Glossary and an Index are provided.

Laboratory codes and schedules are grouped by parameters that are measured together in the NWQL. In cases where more than one analytical method is offered for a single element or compound, different laboratory codes are given.

For information about the methods used for the analytical services offered in this catalog, refer to the section entitled "Selected References."

For more detailed information or discussions, the reader is advised to contact the NWQL. Laboratory contacts are listed in table 1; abbreviations, classifications, and units for all tables are listed in table 2 in place of headnotes. This catalog supersedes the 1993 National Water Quality Laboratory Services Catalog (C.A. Watterson and A.T. Kashuba, U.S. Geological Survey, written commun., 1993, NWQL Technical Memorandum 93.04). 


\section{ACKNOWLEDGMENTS}

Thanks are extended to the authors and editors of all previous editions of the National Water Quality Laboratory Services Catalog. Over the years, many people have devoted their time and knowledge toward making each succeeding catalog more comprehensive. Appreciation is extended to Steve Glodt for compiling the National Water-Quality Assessment Program (NAWQA) data for inclusion in the 1994 catalog. 
Table 1.--National Water Quality Laboratory contacts

\begin{tabular}{ll}
\hline \multicolumn{1}{c}{ Contact } & \multicolumn{1}{c}{ Title (EDOC address) } \\
\hline \hline NWQL management staff & \\
Pete Rogerson & Chief, Branch of Analytical Services (ROGERSON) \\
Bob Williams & Assistant Branch Chief (BOBWIL) \\
Al Driscoll & Acting Chief, Quality Management Program (DRISCOLL) \\
Merle Shockey & Chief, Inorganic Program (MSHOCKEY) \\
Mark Burkhardt & Acting Chief, Organic Program (MRBURK) \\
Mark Sandstrom & Chief, Methods Research and Development Program \\
& (SANDSTRO) \\
Debbie Treseder & Chief, Administrative Services Unit (TRESEDER) \\
Carlos Arozarena & Safety Officer, NWQL and Central Region (CARLOS) \\
Linda Pratt & Chief, Laboratory Operations Program (LKPRATT)
\end{tabular}

\section{Contacts for specific questions}

Department of Defense Environmental Contamination (DODEC)

Dorothy Walker (DMWALKER)

Computer Services Unit updates,

DENADP

communications, and reloads

Bottles/ampules from NWQL

Will Lanier (WDLANIER or DENSUPPL)

Field quality-assurance/quality-control

Bruce Darnel (BRDARNEL)

materials (blank water and spikes)

Mark Sandstrom

Inorganic production and program activities

Merle Shockey (DENINORG)

Inorganic quality-control activities

Merle Shockey or DENQC

Laboratory catalog

Linda Pratt

Pat Timme (PJTIMME)

Laboratory Automation Data System

Sandy Turner (STURNER)

(LADS)

Log-in

Peggy O'Mara-Lopez (PGOMARA or DENLOG)

Methods development and special

Mark Sandstrom activities 
Table 1.--National Water Quality Laboratory contacts--Continued

\begin{tabular}{ll}
\hline \multicolumn{1}{c}{ Contact } & \multicolumn{1}{c}{ Title (EDOC address) } \\
\hline \hline Contacts for specific questions & \\
$\begin{array}{l}\text { National Water-Quality Assessment } \\
\text { Program }\end{array}$ & $\begin{array}{l}\text { Steve Glodt (SRGLODT) (NAWQADAT) } \\
\text { Kim Pirkey (KDPIRKEY) }\end{array}$ \\
New schedules/parameter codes & Steve Glodt (SRGLODT) \\
$\begin{array}{l}\text { Organic production and program } \\
\text { activities }\end{array}$ & Mark Burkhardt (MRBURK or DENORG) \\
$\begin{array}{l}\text { Priority and custom/ } \\
\text { specific constituent }\end{array}$ & Merle Shockey--Inorganic \\
$\begin{array}{l}\text { Quality-control reanalysis requests } \\
\text { for districts }\end{array}$ & Mark Burkhardt--Organic \\
Radiochemical contracts & DENQC \\
Custom analysis & Ann Mullin (AHMULLIN) \\
General assistance & Mark Sandstrom \\
\hline
\end{tabular}




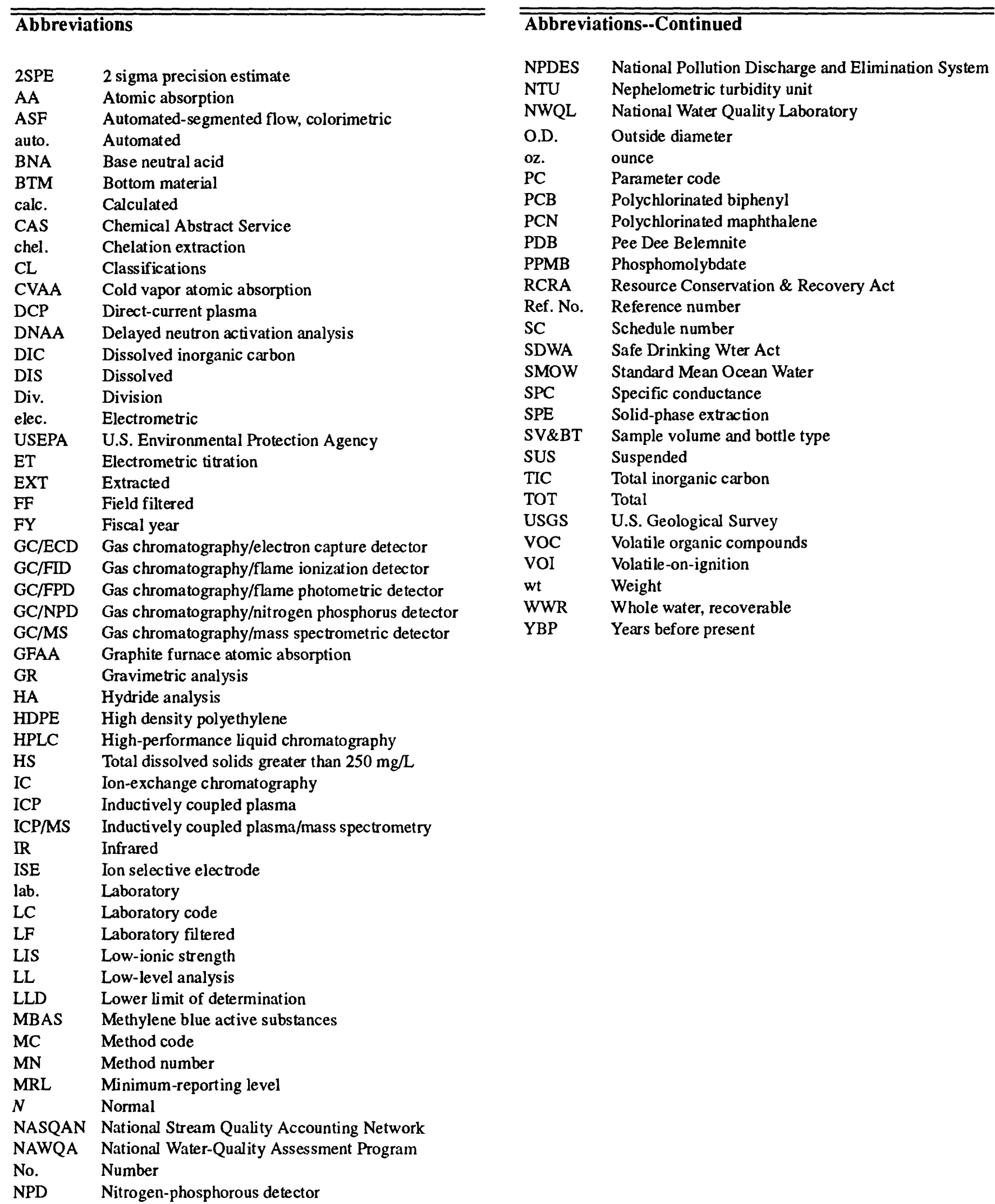


Table 2.--Abbreviations, classifications, and units--Continued

\begin{tabular}{|c|c|}
\hline$\overline{\text { Signs a }}$ & $\overline{\text { symbols }}$ \\
\hline -- & Information not available \\
\hline @ & At \\
\hline \pm & Plus or minus \\
\hline$>$ & Greater than \\
\hline$<$ & Less than \\
\hline$\geq$ & Greater than or equal to \\
\hline $\bar{s}$ & Less than or equal to \\
\hline Units & leasurement \\
\hline$\%$ & percent \\
\hline $\operatorname{deg} C$ & degree Celsius \\
\hline $\mathbf{g}$ & gram \\
\hline gal & gallon \\
\hline $\mathrm{g} / \mathrm{kg}$ & gram per kilogram \\
\hline $\mathrm{g} / \mathrm{m}^{2}$ & gram per square meter \\
\hline $\mathrm{g} / \mathrm{mL}$ & gram per milliliter \\
\hline in. & inch \\
\hline $\mathrm{L}$ & liter \\
\hline $\mathbf{m g}$ & milligram \\
\hline $\mathrm{mg} / \mathrm{kg}$ & milligram per kilogram \\
\hline $\mathrm{mg} / \mathrm{L}$ & milligram per liter \\
\hline $\mathrm{mg} / \mathrm{m}^{2}$ & milligram per square meter \\
\hline $\mathrm{mL}$ & milliliter \\
\hline $\mathrm{mm}$ & millimeter \\
\hline $\mathrm{pt}$ & pint \\
\hline $\mathrm{pCi} / \mathrm{g}$ & picocurie per gram \\
\hline $\mathrm{pCi} / \mathrm{L}$ & picocurie per liter \\
\hline$\mu \mathrm{g} / \mathrm{g}$ & microgram per gram \\
\hline$\mu \mathrm{g} / \mathrm{kg}$ & microgram per kilogram \\
\hline$\mu \mathrm{g} / \mathrm{L}$ & microgram per liter \\
\hline$\mu \mathrm{L}$ & microliter \\
\hline$\mu \mathrm{m}$ & micrometer \\
\hline umol & micromole \\
\hline$\mu \mathrm{S} / \mathrm{cm}$ & $\begin{array}{l}\text { microsiemens per centimeter } \\
\text { at } 25 \text { degrees Celsius }\end{array}$ \\
\hline
\end{tabular}




\section{CONTAINERS, SOLUTIONS, AND MISCELLANEOUS ITEMS}

Various containers, solutions, and supplies used in collecting, processing, and preserving samples for subsequent chemical analysis in the NWQL are available from both the NWQL and the Quality Water Service Unit (QWSU) in Ocala, Fla. Orders may be placed with the NWQL (DENSUPPL) or QWSU (OCALAMAN). These supplies need to be used to ensure the integrity of the sample when they are submitted for analysis to the NWQL. Considerable effort goes into ensuring the quality of the supplies though various quality-control (QC) steps in monitoring the contractors' products.

Many WRD customers wish to use these supplies for samples that will not be analyzed by the NWQL. The cost of supplies is not included in the price of the analysis since it would raise the overall cost for those requesting analyses from the Laboratory. Supply prices are based on the cost of purchase, cleaning, and quality assurance, and are billed upon ordering. Due to the high cost of Teflon ${ }^{1}$ containers, they remain the property of the NWQL and are loaned to the requester. Field supplies available from the NWQL and QWSU are listed in table 3.

To order containers and supplies from the NWQL or the QWSU, send an EDOC message to DENSUPPL or OCALAMAN containing the following information:

1. List each item ordered exactly as described in table 3.

2. State the number of units ordered (for example, 5 packs of 25).

3. Give the account number to be charged.

4. Give the name and shipping address.

If this information is not complete, the order will be delayed while the information is gathered by return EDOC or telephone call. The order will be filled as quickly as possible. However, because of circumstances beyond the control of the NWQL and QWSU, some items may be temporarily out of stock, and partial orders may have to be shipped.

'Use of trade and brand names in this report is for identification purposes only and does not constitute endorsement by the U.S.

Geological Survey. 

Table 3.--Field supplies

[Obtain from: D, National Water Quality Laboratory, Denver, Colo.; O, Quality Water Service Unit, Ocala, Fla.]

Containers, solutions, and miscellaneous items Obtain Bottle type/sample from designation $^{1}$

Unit supplied

Ampule, $\mathrm{H}_{3} \mathrm{PO}_{4} / \mathrm{CuSO}_{4}$

Ampule, $\mathrm{HgCl}_{2}-\mathrm{NaCl}, 0.5 \mathrm{~mL}$

O LCO052

25 /pack

(13 mg $\mathrm{HgCl}_{2}, 100 \mathrm{mg} \mathrm{NaCl}$ )

Ampule, nitric acid, $1 \mathrm{~mL}$

O FC, RC

$24 /$ pack

Ampule, nitric acid, $2 \mathrm{~mL}$

Ampule, nitric acid, ultrapure, $1 \mathrm{~mL}$

O FA, RA, RAE, RAH

24 /pack

Ampule/vial, nitric acid, ultrapure, $1 \mathrm{~mL}$ packaged in $3 \mathrm{~mL}$ Teflon vial ${ }^{2}$

Ampule/vial, nitric acid, ultrapure, $1 \mathrm{~mL}$ packaged in $3 \mathrm{~mL}$ Teflon vial ${ }^{2}$

Ampule, nitric acid/potassium

dichromate, $10 \mathrm{~mL}$

O FA, RA, FAR 25 /pack

O FAB, RAB 25 /pack

O FAB, RAB 24 /pack

O FAB, RAB 5 /pack

O FAM, RAM $25 /$ pack

Ampule, sodium hydroxide, $5 N, 5 \mathrm{~mL}$

Ampule, sulfuric acid, $1 \mathrm{~mL}$

Ampule, sulfuric acid, $2 \mathrm{~mL}$

O LCO880, LCO023 $24 /$ pack

O LCO076 25/pack

O LCO127 $25 /$ pack

Analytical service request forms (ASRs)

O All codes

1 each

Ascorbic acid (vitamin C), $5 \mathrm{~g}$

O

1 each

Ascorbic acid (VOC), $5 \mathrm{~g}$,

D

1 each

includes measuring scoop

Bag, bubble, 1 L 6 in. $x 9$ in.

Bag, mesh, 14 in. $x 16$ in.

$\mathrm{O}$

$\mathrm{O}$

0

1 each

Bag, mesh, 16 in. $\times 24$ in.

1 each

1 each

Blank water, inorganic

O

$1 \mathrm{gal}$

Blank water, pesticide

D

4 /case

Blank water, pesticide

D

$1 \mathrm{gal}$

Blank water, VOC

D

$1 \mathrm{gal}$

Blank water, VOC

D

4 /case

Bottle, glass, amber, $1 \mathrm{~L}$, 33-mm neck, baked

D GCC, LC0052, 12 /case

Bottle, glass, amber, $125 \mathrm{~mL}$, LC0127

22-mm neck, raw (Poly-Seal caps

D LC1574, LC0489 24 /case need to be ordered separately) 
Table 3.--Field supplies--Continued

\begin{tabular}{|c|c|c|c|c|}
\hline Containers, solutions, and miscella & eous items & $\begin{array}{c}\text { Obtain } \\
\text { from }\end{array}$ & $\begin{array}{c}\text { Bottle type/sample } \\
\text { designation }^{1}\end{array}$ & Unit supplied \\
\hline $\begin{array}{l}\text { Bottle, glass, amber, } 125 \mathrm{~mL} \text {, } \\
22 \text {-mm neck, baked, with caps }\end{array}$ & & D & $\begin{array}{l}\text { LC0019, LCO076, } \\
\text { LC0113, LC0114, } \\
\text { LC0306, SC1379 }\end{array}$ & $24 /$ case \\
\hline $\begin{array}{l}\text { Bottle, glass, amber, } 1 \mathrm{~L}, 33-\mathrm{mm} \\
\text { neck, raw (Poly-Seal caps } \\
\text { need to be ordered separately) }\end{array}$ & . & D & $\begin{array}{l}\text { LC1043, LC1565 } \\
\text { LC0440, LC0298, } \\
\text { LC1717, LC1718 }\end{array}$ & 12 /case \\
\hline $\begin{array}{l}\text { Bottle, glass, clear, } 1 \mathrm{~L} \text {, } \\
\text { 53-mm neck, baked }\end{array}$ & & D & Fits auto sampler & $12 /$ case \\
\hline $\begin{array}{l}\text { Bottle, glass, clear, } 1 \mathrm{~L} \text {, } \\
89 \text {-mm neck, baked }\end{array}$ & & D & LC1199 & 12 /case \\
\hline $\begin{array}{l}\text { Bottle, glass, clear, } 250 \mathrm{~mL} \text {, } \\
24-\mathrm{mm} \text { neck, acid-rinsed }\end{array}$ & & 0 & FAM, RAM & 24 /case \\
\hline $\begin{array}{l}\text { Bottle, glass, clear, } 250 \mathrm{~mL} \text {, } \\
\text { 24-mm neck, baked }\end{array}$ & & D & -- & $24 /$ case \\
\hline $\begin{array}{l}\text { Bottle, glass, clear, } 500 \mathrm{~mL} \text {, } \\
89-\mathrm{mm} \text { neck, baked }\end{array}$ & & D & BGC & $12 /$ case \\
\hline $\begin{array}{l}\text { Bottle, glass, clear, } 60 \mathrm{~mL} \text {, } \\
20 \text {-mm neck, raw (Poly-Seal } \\
\text { cap included) }\end{array}$ & & 0 & LC1574, LC0489 & $24 /$ case \\
\hline $\begin{array}{l}\text { Bottle, polyethylene, brown, } \\
125 \mathrm{~mL}, 28 \text {-mm neck, raw (caps } \\
\text { need to be ordered separately) }\end{array}$ & & 0 & FC, RC & $100 /$ repack \\
\hline $\begin{array}{l}\text { Bottle, polyethylene, brown, } \\
125 \mathrm{~mL}, 28 \text {-mm neck, raw (caps } \\
\text { need to be ordered separately) }\end{array}$ & & 0 & $\mathrm{FC}, \mathrm{RC}$ & $660 /$ case \\
\hline $\begin{array}{l}\text { Bottle, polyethylene, natural, } \\
1 \mathrm{~L}, 28 \text {-mm neck, acid rinsed } \\
\text { with clear cap }\end{array}$ & & $\mathrm{O}$ & FAR, RUR & $144 /$ case \\
\hline $\begin{array}{l}\text { Bottle, polyethylene, natural, } \\
1 \mathrm{~L}, 28 \text {-mm neck, raw }\end{array}$ & -- & 0 & FU, RU & $144 /$ case \\
\hline $\begin{array}{l}\text { Bottle, polyethylene, natural, } \\
1 \mathrm{~L}, 28-\mathrm{mm} \text { neck, raw (tritium) } \\
\text { (Poly-Seal cap included) }\end{array}$ & & $\mathrm{o}$ & LC1043, LC1565 & each \\
\hline $\begin{array}{l}\text { Bottle, polyethylene, natural, } \\
125 \mathrm{~mL}, 28 \text {-mm neck, } \\
\text { acid-rinsed with clear cap }\end{array}$ & & 0 & -- & 660 /case \\
\hline
\end{tabular}


Table 3.--Field supplies--Continued

\begin{tabular}{|c|c|c|c|}
\hline Containers, solutions, and miscellaneous items & $\begin{array}{c}\text { Obtain } \\
\text { from }\end{array}$ & $\begin{array}{c}\text { Bottle type/sample } \\
\text { designation }^{1}\end{array}$ & Unit supplied \\
\hline $\begin{array}{l}\text { Bottle, polyethylene, natural, } \\
125 \mathrm{~mL}, 28-\mathrm{mm} \text { neck, raw (caps } \\
\text { need to be ordered separately) }\end{array}$ & 0 & -- & 100 /repack \\
\hline $\begin{array}{l}\text { Bottle, polyethylene, natural } \\
125 \mathrm{~mL}, 28 \text {-mm neck, raw (black } \\
\text { plastic caps need to be ordered } \\
\text { separately) }\end{array}$ & $\mathrm{O}$ & $\begin{array}{l}\text { LC0050, LC0452 } \\
\text { (LC0452 must have } \\
\text { Poly-Seal cap) }\end{array}$ & 660 /case \\
\hline $\begin{array}{l}\text { Bottle, polyethylene, natural, } \\
125 \mathrm{~mL}, 28-\mathrm{mm} \text { neck, raw (black } \\
\text { plastic caps need to be ordered } \\
\text { separately) }\end{array}$ & 0 & $\begin{array}{l}\text { LC0050, LC0452 } \\
\text { (LC0452 must have } \\
\text { Poly-Seal cap) }\end{array}$ & $660 /$ case \\
\hline $\begin{array}{l}\text { Bottle, polyethylene, natural, } 250 \mathrm{~mL} \text {, } \\
28 \text {-mm neck, acid-rinsed with clear cap }\end{array}$ & $\mathrm{O}$ & FA, RA, RAE, RAH & $\begin{array}{l}15 \text { /case } \\
\text { (with } \\
\text { Ziploc } \\
\text { bag) }\end{array}$ \\
\hline $\begin{array}{l}\text { Bottle, polyethylene, natural, } \\
250 \mathrm{~mL}, 28 \text {-mm neck, acid- } \\
\text { rinsed with clear cap }\end{array}$ & 0 & & $\begin{array}{l}225 \text { /case } \\
\text { (15 per } \\
\text { Ziploc } \\
\text { bag) }\end{array}$ \\
\hline $\begin{array}{l}\text { Bottle, polyethylene, natural, } \\
250 \mathrm{~mL}, 28-\mathrm{mm} \text { neck, raw (caps } \\
\text { need to be ordered separately) }\end{array}$ & $\mathrm{O}$ & $\begin{array}{l}\text { FU, RCB, RU, LC0023 } \\
\text { LC0089, LC0880, } \\
\text { LC0460 (LC0460- } \\
\text { must have Poly-Seal } \\
\text { cap) }\end{array}$ & $320 /$ case \\
\hline $\begin{array}{l}\text { Bottle, polyethylene, natural, } \\
500 \mathrm{~mL}, 28-\mathrm{mm} \text { neck, acid- } \\
\text { rinsed with clear cap }\end{array}$ & $\mathrm{O}$ & $\mathrm{FA}, \mathrm{RA}$ & 228 /case \\
\hline $\begin{array}{l}\text { Bottle, polyethylene, natural, } \\
500 \mathrm{~mL}, 28 \text {-mm neck, raw (caps } \\
\text { need to be ordered separately) }\end{array}$ & 0 & $\begin{array}{l}\text { FU, RU, } \\
\text { LC0169, LC0624 }\end{array}$ & $228 /$ case \\
\hline $\begin{array}{l}\text { Bottle, polypropylene, natural, } \\
500 \mathrm{~mL}, 89 \text {-mm neck, acid- } \\
\text { rinsed with clear cap }\end{array}$ & & NAWQA & 1 each \\
\hline $\begin{array}{l}\text { Bottle, polypropylene, natural, } \\
500 \mathrm{~mL}, 89 \text {-mm neck, raw (caps } \\
\text { need to be ordered separately) }\end{array}$ & $\mathrm{o}$ & $\mathrm{CC}, \mathrm{CU}$ & 1 each \\
\hline $\begin{array}{l}\text { Bottle, Teflon, natural, } 250 \mathrm{~mL} \text {, rental, } \\
\text { usage and cleaning fee, acid-rinsed with } \\
\text { cap (bottle is to be returned to laboratory }\end{array}$ & 0 & FAB, RAB & 1 each \\
\hline
\end{tabular}


Table 3.--Field supplies--Continued

\begin{tabular}{|c|c|c|c|}
\hline Containers, solutions, and miscellaneous items & $\begin{array}{c}\text { Obtain } \\
\text { from }\end{array}$ & $\begin{array}{c}\text { Bottle type/sample } \\
\text { designation }^{1}\end{array}$ & Unit supplied \\
\hline Caps, melamine, green, $89 \mathrm{~mm}$ & $\mathrm{O}$ & $\begin{array}{l}\text { Bottle, polyethylene, } \\
\text { wide-mouth sediment }\end{array}$ & $12 /$ case \\
\hline Caps, melamine, green, $89 \mathrm{~mm}$ & $\mathrm{O}$ & $\begin{array}{l}\text { Bottle, polyethylene, } \\
\text { wide-mouth sediment }\end{array}$ & 1 each \\
\hline Caps, metal, size 22 & $\mathrm{O}$ & Bottle, glass $4 \mathrm{oz}$ & 1 each \\
\hline Caps, metal, size 33 & $\mathrm{O}$ & Bottle, glass $32 \mathrm{oz}$ & 1 each \\
\hline Caps, metal, size 53 & $\mathrm{O}$ & $\begin{array}{l}\text { Bottle, glass } 32 \mathrm{oz} \text {, } \\
\text { wide-mouth packer }\end{array}$ & 1 each \\
\hline Caps, metal, size 89 & $\mathrm{O}$ & Bottle, glass $16 \mathrm{oz}$ & 1 each \\
\hline Caps, plastic, black, 28-mm & $\mathrm{O}$ & $\begin{array}{l}\text { Bottle, polyethylene, } \\
\text { raw }\end{array}$ & 1,200 each \\
\hline Caps, plastic, black, 28-mm & $\mathrm{O}$ & $\begin{array}{l}\text { Bottle, polyethylene, } \\
\text { raw }\end{array}$ & 100 /repack \\
\hline Caps, Poly-Seal cone insert, 22-mm & 0 & Bottle, glass, $125 \mathrm{~mL}$ & 1 each \\
\hline Caps, Poly-Seal cone insert, 28-mm & $\mathrm{O}$ & $\begin{array}{l}\text { Bottle, } \\
\text { polyethylene } 4,8,16\end{array}$ & 1 each \\
\hline Caps, Poly-Seal cone insert, 33-mm & $\mathrm{O}$ & $\begin{array}{l}\text { Bottle, } \\
\text { polyethylene } 4,8,16\end{array}$ & 1 each \\
\hline Cartridge, SPE, C-18 & D & SC 2010 & 1 each \\
\hline Cartridge, SPE, Carbopak-B & D & SC 2051 & 1 each \\
\hline Cartridge, SPE, Adapter Kit & D & SC 2051 & 1 each \\
\hline Cartridge, SPE, flow-control valve & D & & 1 each \\
\hline Filters, silver or biological, or both & $\mathrm{O}$ & -- & 1 each \\
\hline $\begin{array}{l}\text { Hydrodhloric acid, concentrated, } 30-\mathrm{ml} \text { Teflon } \\
\text { bottle for NAWQA VOC preservation }\end{array}$ & D & & 1 each \\
\hline $\begin{array}{l}\text { Hydrochloric acid, diluted } 1: 4 \text { with water, } \\
30-\mathrm{ml} \text { Teflon bottle for acrolein/acrlonitril } \\
\text { VOC preservation }\end{array}$ & D & & 1 each \\
\hline Kit, media, agar, fecal coliform & 0 & -- & $15 /$ kit \\
\hline Kit, media, agar, fecal streptococci & $\mathrm{O}$ & -- & $15 / \mathrm{kit}$ \\
\hline Kit, media, agar, total coliform & $\mathrm{O}$ & -- & $15 /$ kit \\
\hline Kit, organic field spiking (mixtures extra) ${ }^{3}$ & $\mathrm{D}$ & & 1 each \\
\hline $\begin{array}{l}\text { Kit, capillary glass bores, } \\
\text { baked replacement, } 100 \mu \mathrm{L}\end{array}$ & D & -- & $30 /$ kit \\
\hline
\end{tabular}




\begin{tabular}{|c|c|c|c|}
\hline Containers, solutions, and miscellaneous items & $\begin{array}{c}\text { Obtain } \\
\text { from }\end{array}$ & $\begin{array}{c}\text { Bottle type/sample } \\
\text { designation }^{1}\end{array}$ & Unit supplied \\
\hline $\begin{array}{l}\text { Kit, periphyton, chlorophyll } \\
\text { and biomass }\end{array}$ & 0 & CHE & $1 \mathrm{kit}$ \\
\hline Kit, phytoplankton, chlorophyll & $\mathrm{O}$ & CHY & 1 kit \\
\hline $\begin{array}{l}\text { Labels, MRM (merchandise return } \\
\text { mail) 1st or 4th class }\end{array}$ & D & & 25 /pack \\
\hline Labels, SMS (sample management system) & D & -- & $5,000 /$ box \\
\hline Packer, foam, universal, $1 \mathrm{~L}$ & $\mathrm{O}$ & -- & 1 each \\
\hline $\begin{array}{l}\text { pH Buffers }(4.0,7.0,10.0), 1 \mathrm{~L} \\
\text { pH Buffers }(4.0,7.0,10.0), 5 \mathrm{gal} \\
\text { pH Buffers (low ionic), } 1 \mathrm{~L}\end{array}$ & $\begin{array}{l}0 \\
\text { O } \\
\text { O }\end{array}$ & $\begin{array}{l}-- \\
-- \\
--\end{array}$ & $\begin{array}{l}1 \text { each } \\
1 \text { each } \\
1 \text { each }\end{array}$ \\
\hline Radon kits & D & -- & 1 each \\
\hline $\begin{array}{l}\text { Sleeve, foam, } 5 \text { in. } \times 8 \text { in. } \\
\text { Sleeve, foam, } 6 \text { in. } \times 10 \text { in. }\end{array}$ & $\begin{array}{l}0 \\
0\end{array}$ & $\begin{array}{l}-- \\
--\end{array}$ & $\begin{array}{l}1 \text { each } \\
1 \text { each }\end{array}$ \\
\hline Solution, field spike, NAWQA VOC & D & SC 2090 & 1 ampule \\
\hline $\begin{array}{l}\text { Solution, field spike, } \\
\text { NAWQA SC 2001/2010 }\end{array}$ & D & SC 2001/2010 & 1 ampule \\
\hline Solution, field spike, NPDES BNA & D & SC $1383 / 1385$ & 1 ampule \\
\hline Solution, field spike, NPDES OCP & D & SC 1608 & 1 ampule \\
\hline Solution, field spike, NPDES VOC & D & SC 1380 & 1 ampule \\
\hline Solution, field spike (2050/2051) & D & SC 2050//2051 & 1 ampule \\
\hline Solution, field spike, triazine $(125 \mathrm{~mL})$ & D & SC 1379 & 1 ampule \\
\hline Solution, sodium thiosulfate, $0.025 \mathrm{~N}$ & 0 & & $1 \mathrm{~L}$ \\
\hline $\begin{array}{l}\text { Solution, specific conductance } \\
(10-20 \mu \mathrm{S} / \mathrm{cm})\end{array}$ & 0 & & $1 \mathrm{~L}$ \\
\hline $\begin{array}{l}\text { Solution, specific conductance } \\
(40-60 \mu \mathrm{S} / \mathrm{cm})\end{array}$ & 0 & & $1 \mathrm{~L}$ \\
\hline $\begin{array}{l}\text { Solution, specific conductance } \\
(80-120 \mu \mathrm{S} / \mathrm{cm})\end{array}$ & 0 & & $1 \mathrm{~L}$ \\
\hline $\begin{array}{l}\text { Solution, specific conductance } \\
(200 / 250 \mu \mathrm{S} / \mathrm{cm})\end{array}$ & 0 & & $1 \mathrm{~L}$ \\
\hline
\end{tabular}


Table 3.--Field supplies--Continued

\begin{tabular}{|c|c|c|c|}
\hline Containers, solutions, and miscellaneous items & $\begin{array}{c}\text { Obtain } \\
\text { from }\end{array}$ & $\begin{array}{c}\text { Bottle type/sample } \\
\text { designation }^{1}\end{array}$ & Unit supplied \\
\hline $\begin{array}{l}\text { Solution, specific conductance } \\
(300-450 \mu \mathrm{S} / \mathrm{cm})\end{array}$ & 0 & & $1 \mathrm{~L}$ \\
\hline $\begin{array}{l}\text { Solution, specific conductance } \\
(500-750) \mu \mathrm{S} / \mathrm{cm})\end{array}$ & 0 & & $1 \mathrm{~L}$ \\
\hline $\begin{array}{l}\text { Solution, specific conductance } \\
(800-1,100 \mu \mathrm{S} / \mathrm{cm})\end{array}$ & 0 & & $1 \mathrm{~L}$ \\
\hline $\begin{array}{l}\text { Solution, specific conductance } \\
(1,800-2,100 \mu \mathrm{S} / \mathrm{cm})\end{array}$ & 0 & & $1 \mathrm{~L}$ \\
\hline $\begin{array}{l}\text { Solution, specific conductance } \\
(2,500-3,000 \mu \mathrm{S} / \mathrm{cm})\end{array}$ & 0 & & $1 \mathrm{~L}$ \\
\hline $\begin{array}{l}\text { Solution, specific conductance } \\
(4,000-5,000 \mu \mathrm{S} / \mathrm{cm})\end{array}$ & 0 & & $1 \mathrm{~L}$ \\
\hline $\begin{array}{l}\text { Solution, specific conductance } \\
(7,500-8,500 \mu \mathrm{S} / \mathrm{cm})\end{array}$ & 0 & & $1 \mathrm{~L}$ \\
\hline $\begin{array}{l}\text { Solution, specific conductance } \\
(10,500-11,500 \mu \mathrm{S} / \mathrm{cm})\end{array}$ & 0 & & $1 \mathrm{~L}$ \\
\hline Solution, sulfuric acid, $0.0164 N$ (approximate) & $\mathrm{O}$ & & $1 \mathrm{~L}$ \\
\hline $\begin{array}{l}\text { Surrogate mixture } \\
\text { Surrogate mixture }\end{array}$ & $\begin{array}{l}\text { D } \\
\text { D }\end{array}$ & $\begin{array}{l}\text { SC } 2010 \\
\text { SC } 2051\end{array}$ & $\begin{array}{l}2 \mathrm{~mL} \\
3 \mathrm{~mL}\end{array}$ \\
\hline Trip blanks (VOC) & D & $\begin{array}{l}8 \text { filled vials } \\
\text { added to a case of } \\
\text { ordered VOC vials }\end{array}$ & $8 /$ case \\
\hline Vial, glass, amber septum, $40 \mathrm{~mL}$ & $\mathrm{O}$ & VOC & $72 /$ box \\
\hline $\begin{array}{l}\text { Water, dilution, buffered, sterile, } \\
\text { bacteriological samples, } 99 \mathrm{~mL}\end{array}$ & 0 & -- & $12 /$ box \\
\hline $\begin{array}{l}\text { Water, rinse, buffered, sterile, } \\
\text { bacteriological samples, } 250 \mathrm{~mL}\end{array}$ & 0 & -- & $8 /$ pack \\
\hline $\begin{array}{l}\text { Wrench set, plastic for } 3 \mathrm{~mL} \text { Teflon vial } \\
\text { containing ultrapure nitric acid }\end{array}$ & 0 & & $1 \mathrm{set}$ \\
\hline Zinc acetate, $25 \mathrm{~g}$ & 0 & LC0089 & $1 \mathrm{jar}$ \\
\hline
\end{tabular}

See table 4.

${ }^{2} \mathrm{~A} \$ 4.00$ credit will be given upon return of each Teflon vial after the preservative is used.

${ }^{3}$ Return of kits will result in credit for account.

${ }^{4}$ Surrogate mixtures have a shelf-life of six months and should not be ordered more than two months prior to intended use. 


\section{SAMPLE PROCESSING}

To ensure the quality of data produced by the NWQL, sampling protocols need to be followed, and samples need to be shipped as expeditiously as possible. In addition, samples for the requested analyses need to be appropriately preserved and stored in suitable containers. To ensure the integrity of the samples, they should be submitted using quality-assured containers and preservatives supplied by the NWQL or QWSU.

Containers available from the NWQL or QWSU, the recommended sample treatment prior to packing, and the preferred sample preservation technique are listed in table 4.

WRD personnel are urged to review Office of Water Quality (OWQ) Technical Memoranda 92.01, "Distilled/Deionized Water for District Operations," 92.02, "Field Preparation of Containers for Aqueous Samples," as well as table 4.

Any bottom-material samples received for inorganic analysis need to be presieved through a $2.0-\mathrm{mm}$ sieve using minimal volume of native water. Unsieved samples will be sieved by the NWQL (using deionized water), and an additional $\$ 55.00$ charge will be made by the Laboratory. Samples will be sieved as time permits; therefore, turnaround time will increase.

All samples submitted to the NWQL for analysis must be accompanied by an Analytical Services Request (ASR) form. To ensure correct processing of samples, all shipments should include ASR forms that refer only to the samples in that shipment. The ASR forms should be filled out completely, and must include information on project chief, current telephone numbers, and the number and type of bottles sent. To expedite data review, inorganic samples should also include information on $\mathrm{pH}$, specific conductance, and, when available, field alkalinity. For more information on shipping to NWQL, WRD personnel are encouraged to review OWQ Memo 92.06, "Report of Committee on Sample Shipping Integrity and Cost."

NOTE: Protocols for a national program supersede these suggestions. For example, inorganic analysis of sediments for NAWQA require on-site sieving of the sample through $63-\mu \mathrm{m}$ mesh. 

Table 4.--Sample designations, containers, and treatments

\begin{tabular}{cccc}
\hline $\begin{array}{c}\text { Sample } \\
\text { desig- } \\
\text { nation }\end{array}$ & $\begin{array}{c}\text { Con- } \\
\text { tainer } \\
\text { size }\end{array}$ & Container type & Treatment and preservation \\
\hline
\end{tabular}

\begin{tabular}{|c|c|c|}
\hline \multicolumn{3}{|c|}{ Biological determinations } \\
\hline $\mathrm{CHE}$ & Glass jar, wide-mouth & $\begin{array}{l}\text { Place strip in jar. Wrap jar in aluminum foil, freeze with dry ice, } \\
\text { and ship expeditiously. }\end{array}$ \\
\hline CHY & Glass vial & $\begin{array}{l}\text { Collect on glass filter. Record volume filtered. Place filter in } \\
\text { vial and wrap vial in aluminum foil. Freeze with dry ice and } \\
\text { ship expeditiously. }\end{array}$ \\
\hline
\end{tabular}

\section{Inorganic determinations: Water, water-sediment and bottom-material samples}

CC 1 pt $\quad$ Polypropylene bottle, wide-mouth

CU 1 pt Polyethylene bottle, wide-mouth

FA 250 or Polyethylene bottle, acid-rinsed

$500 \mathrm{~mL}$

FAB $\quad 250 \mathrm{~mL}$ Teflon bottle, acid-rinsed

FAM $\quad 250 \mathrm{~mL} \quad$ Glass bottle, acid-rinsed

FC $\quad 125 \mathrm{~mL} \quad$ Brown polyethylene bottle, field-rinsed

FU 250 or Polyethylene bottle, field-rinsed $500 \mathrm{~mL}$

RA 250 or Polyethylene bottle, acid-rinsed $500 \mathrm{~mL}$

RAB $250 \mathrm{~mL}$ Teflon bottle, acid-rinsed

RAE $\quad 250 \mathrm{~mL}$ Polyethylene bottle, acid-rinsed

RAH $250 \mathrm{~mL}$ Polyethylene bottle, acid-rinsed

RAM $250 \mathrm{~mL}$ Glass bottle, acid-rinsed

RC $125 \mathrm{~mL}$ Brown polyethylene bottle, field-rinsed

RCB $250 \mathrm{~mL}$ Polyethylene bottle, field-rinsed

RU 250 or Polyethylene bottle, field-rinsed $500 \mathrm{~mL}$

LC0023 $250 \mathrm{~mL}$ Polyethylene bottle, field-rinsed

LC0050 $125 \mathrm{~mL}$ Polyethylene bottle, field-rinsed
Field sieve through $2-\mathrm{mm}$ plastic sieve using native water. Field sieve through $2-\mathrm{mm}$ plastic sieve using native water. Filter through $0.45-\mu \mathrm{m}$ filter; use filtered sample to rinse containers and acidify sample with $\mathrm{HNO}_{3}$ to $\mathrm{pH}<2$; . container size dependent on laboratory schedule.

Filter through $0.45-\mu \mathrm{m}$ filter; use filtered sample to rinse containers and acidify collected sample with ultrapure $\mathrm{HNO}_{3}$ to $\mathrm{pH}<2$.

Filter through $0.45-\mu \mathrm{m}$ filter; use filtered sample to rinse containers and acidify collected sample with 1 ampule of $\mathrm{HNO}_{3} / \mathrm{K}_{2} \mathrm{Cr}_{2} \mathrm{O}_{7}$.

Filter through $0.45-\mu \mathrm{m}$ filter; use filtered sample to rinse containers, add ampule $\mathrm{HgCl}_{2}$ solution to collected sample, chill and maintain at $4 \mathrm{deg} \mathrm{C}$, ship as soon as possible.

Filter through 0.45 - $\mu \mathrm{m}$ filter; using filtered sample to rinse containers; container size dependent on laboratory schedule.

Use unfiltered sample to rinse bottles, then acidify collected sample with $\mathrm{HNO}_{3}$ to $\mathrm{pH}<2$; container size dependent on laboratory schedule.

Use unfiltered sample to rinse bottles, then acidify collected sample with ultrapure $\mathrm{HNO}_{3}$ to $\mathrm{pH}<2$. Use unfiltered sample to rinse bottles, then acidify collected sample with $\mathrm{HNO}_{3}$ to $\mathrm{pH}<2$.

Use unfiltered sample to rinse bottles, then acidify collected sample with $\mathrm{HNO}_{3}$ to $\mathrm{pH}<2$.

Use unfiltered sample to rinse bottles, then acidify collected sample with 1 ampule of $\mathrm{HNO}_{3} / \mathrm{K}_{2} \mathrm{Cr}_{2} \mathrm{O}_{7}$. Use unfiltered sample to rinse bottles, then add 1 ampule $\mathrm{HgCl}_{2}$ solution to collected sample, chill and maintain at $4 \mathrm{deg} C$; ship as soon as possible. Use unfiltered sample to rinse bottles, then chill collected sample and maintain at $4 \mathrm{deg} \mathrm{C}$; ship as soon as possible.

Use unfiltered sample to rinse bottles; container size dependent on laboratory schedule.

Use unfiltered sample to rinse bottles; then add $\mathrm{NaOH}$ to $\mathrm{pH}>12$ to collected sample, chill and maintain at $4 \mathrm{deg}$. Use unfiltered sample to rinse bottles. 
Table 4.--Sample designations, containers, and treatments--Continued

\begin{tabular}{|c|c|c|c|}
\hline $\begin{array}{l}\text { Sample } \\
\text { desig- } \\
\text { nation }\end{array}$ & $\begin{array}{l}\text { Con- } \\
\text { tainer } \\
\text { size }\end{array}$ & Container type & Treatment and preservation \\
\hline \multicolumn{4}{|c|}{ Inorganic determinations: Water, water-sediment and bottom-material samples--Continued } \\
\hline LC0076 & $125 \mathrm{~mL}$ & Glass bottle & $\begin{array}{l}\text { Bottle baked at } 450 \mathrm{deg} \mathrm{C} \text { by laboratory. Add } \mathrm{H}_{2} \mathrm{SO}_{4} \text { to } \mathrm{pH}<2 \text {; } \\
\text { chill and maintain at } 4 \mathrm{deg} \text { C. DO NOT RINSE BOTTLE. }\end{array}$ \\
\hline LC0089 & $250 \mathrm{~mL}$ & Polyethylene bottle, field-rinsed & $\begin{array}{l}\text { Use unfiltered sample to rinse bottles; then add } 0.5 \mathrm{~g} \\
\text { zinc acetate to the collected sample. }\end{array}$ \\
\hline LC0169 & $500 \mathrm{~mL}$ & Polyethylene bottle, field-rinsed & Use unfiltered sample to rinse bottles. \\
\hline LC0880 & $250 \mathrm{~mL}$ & Polyethylene bottle, field-rinsed & $\begin{array}{l}\text { Filter through } 0.45-\mu \mathrm{m} \text { filter; using filtered sample to rinse } \\
\text { bottles; then add } \mathrm{NaOH} \text { to } \mathrm{pH}>12 \text { to collected sample, } \\
\text { chill and maintain at } 4 \text { deg } \mathrm{C} \text {; ship as soon as possible. }\end{array}$ \\
\hline
\end{tabular}

\section{Organic determinations: Water, water-sediment and bottom-material samples}

CC $\quad 1 \mathrm{pt} \quad$ Polyethylene bottle, wide-mouth

BGC $1 \mathrm{~L} \quad$ Glass bottle, wide-mouth

DOC $\quad 125 \mathrm{~mL} \quad$ Glass bottle, amber

GCC $\quad 1 \mathrm{~L} \quad$ Glass bottle, amber

RCB $250 \mathrm{~mL}$ Polyethylene bottle

SOC Petri dish

TOC $\quad 125 \mathrm{~mL} \quad$ Glass bottle, amber

VOC $\quad 40 \mathrm{~mL} \quad$ Glass septum vial, amber

LC0019 $125 \mathrm{~mL} \quad$ Glass bottle, amber
Field sieve through 2-mm plastic sieve using native water. Bottle baked at $450 \mathrm{deg} C$ by laboratory. DO NOT RINSE BOTTLE. Chill and maintain at $4 \mathrm{deg} C$; ship as soon as possible.

Bottle baked at 450 deg $C$ by laboratory. DO NOT RINSE BOTTLE BUT RINSE FILTER WITH ORGANIC FREE WATER. Filter sample using silver filter, chill and maintain at $4 \mathrm{deg} C$; ship as soon as possible. Filter may be retained for $\mathrm{LC} 0305$.

Bottle baked at $450 \mathrm{deg} C$ by laboratory. DO NOT

RINSE BOTTLE. Chill sample and maintain at $4 \mathrm{deg} C$; ship as soon as possible.

Use unfiltered sample to rinse bottles, then chill collected sample and maintain at $4 \mathrm{deg} C$; ship as soon as possible. RINSE FILTER WITH ORGANIC-FREE WATER.

Retain sample on silver filter, chill and maintain at 4 $\operatorname{deg} \mathrm{C}$; ship as soon as possible. Record volume filtered on Analytical Services Request formand on Petri dish Bottle baked at $450 \mathrm{deg} C$ by laboratory. DO NOT RINSE BOTTLE. Chill sample and maintain at 4 $\operatorname{deg} C$; ship as soon as possible.

DO NOT RINSE BOTTLE. Exclude air bubbles by completely filling vial. Protect sample from sunlight, chill and maintain at $4 \mathrm{deg} \mathrm{C}$; ship as soon as possible. For SC 1306 no preservative is required. For SC 1378 each vial is preserved to $\mathrm{pH}<2$ with two drops of $1: 1 \mathrm{HCl}: \mathrm{H}_{2} \mathrm{O}$. NPDES sampling, preserve to $\mathrm{pH}<2$ with two drops of $1: 1 \mathrm{HCl}: \mathrm{H}_{2} \mathrm{O}$ in each vial. If free chlorine is present, add $25 \mathrm{mg}$ of ascorbic acid to each vial in addition to the HCL (for drinking-water or wastewater treatment). Bottle baked at $450 \mathrm{deg} C$ by laboratory. DO NOT RINSE BOTTLE. Chill sample and maintain at $4 \mathrm{deg} C$; ship as soon as possible. 
Table 4.--Sample designations, containers, and treatments--Continued

\begin{tabular}{|c|c|c|c|}
\hline $\begin{array}{l}\text { Sample } \\
\text { desig- } \\
\text { nation }\end{array}$ & $\begin{array}{l}\text { Con- } \\
\text { tainer } \\
\text { size }\end{array}$ & Container type & Treatment and preservation \\
\hline \multicolumn{4}{|c|}{ Organic determinations: Water, water-sediment, and bottom-material samples--Continued } \\
\hline LC0127 & $1 \mathrm{~L}$ & Oil and Grease bottle, amber & $\begin{array}{l}\text { Bottle baked. DO NOT RINSE BOTTLE. Leave small } \\
\text { air space. Add } 2.0 \mathrm{~mL} \mathrm{H}_{2} \mathrm{SO}_{4} \text { to } 1 \mathrm{~L} \text { (to } \mathrm{pH} \text { 2). Chill and } \\
\text { maintain at } 4 \text { deg C; ship as soon as possible. }\end{array}$ \\
\hline SC1379 & $125 \mathrm{~mL}$ & Glass bottle, amber & $\begin{array}{l}\text { Bottle baked at } 450 \text { deg } C \text { by laboratory. DO NOT RINSE } \\
\text { BOTTLE. Field filter through } 0.7-\mu \mathrm{m} \text { disposable in-line } \\
\text { filter. Chill and maintain at } 4 \text { deg C; ship as soon as possible. }\end{array}$ \\
\hline
\end{tabular}

\section{Radiochemical: Water, water-sediment and bottom-material samples}

\begin{tabular}{|c|c|c|c|}
\hline CUR & $1 \mathrm{pt}$ & Polyethylene bottle, wide-mouth & Untreated. \\
\hline FAR & $1 \mathrm{~L}$ & Polyethylene bottle, acid-rinsed & Filter through $0.45-\mu \mathrm{m}$ filter and acidify with $\mathrm{HNO}_{3}$ to $\mathrm{pH}<2$. \\
\hline RAR & $1 \mathrm{~L}$ & Polyethylene bottle, acid-rinsed & Acidify with $\mathrm{HNO}_{3}$ to $\mathrm{pH}<2$. \\
\hline RUR & $1 \mathrm{~L}$ & Polyethylene bottle, acid-rinsed & Untreated. \\
\hline SUR & & Petri dish or centrifuge tube & Field filter or centrifuge until required sediment is achieved. \\
\hline LC0452 & $125 \mathrm{~mL}$ & $\begin{array}{l}\text { Polyethylene bottle; must have } \\
\text { polyethylene seal cap }\end{array}$ & Untreated. \\
\hline LC0460 & $250 \mathrm{~mL}$ & $\begin{array}{l}\text { Polyethylene bottle; must have } \\
\text { polyethylene seal cap }\end{array}$ & Untreated. \\
\hline LC0490 & $50 \mathrm{~mL}$ & Glass bubbler & Contact NWQL. \\
\hline LC0624 & $500 \mathrm{~mL}$ & $\begin{array}{l}\text { Polyethylene bottle; must have } \\
\text { polyethylene seal cap }\end{array}$ & Untreated. \\
\hline LC1043 & $1 \mathrm{~L}$ & $\begin{array}{l}\text { Glass or high density polyethylene bottle; } \\
\text { must have polyethylene seal cap }\end{array}$ & Untreated \\
\hline LC1565 & $1 \mathrm{~L}$ & $\begin{array}{l}\text { Glass or high density polyethylene } \\
\text { bottle; must have polyethylene } \\
\text { seal cap }\end{array}$ & Untreated. \\
\hline
\end{tabular}

Stable isotope: Water, water-sediment and bottom-material samples

\begin{tabular}{|c|c|c|c|}
\hline LC0298 & $1 \mathrm{~L}$ & $\begin{array}{l}\text { Glass bottle, narrow neck, field-rinsed; } \\
\text { must have polyethylene seal cap }\end{array}$ & Contact NWQL. \\
\hline LC1574 & $\begin{array}{l}125 \text { or } \\
60 \mathrm{~mL}\end{array}$ & $\begin{array}{l}\text { Glass or polye thylene bottle, field-rinsed; } \\
\text { must have polyethylene seal cap }\end{array}$ & $\begin{array}{l}\text { Samples can be filtered or unfiltered. Headspace should be } \\
\text { allowed to prevent problems if sample freezes. Seal with } \\
\text { wax or plastic tape. }\end{array}$ \\
\hline LC0440 & $1 \mathrm{~L}$ & $\begin{array}{l}\text { Glass bottle, narrow neck, field-rinsed; } \\
\text { must have polyethylene seal cap }\end{array}$ & Do not filter, add $50 \mathrm{~mL} \mathrm{SrCl}_{2}$, seal with tape. \\
\hline LC0489 & $\begin{array}{l}125 \text { or } \\
60 \mathrm{~mL}\end{array}$ & $\begin{array}{l}\text { Glass or Polyethylene bottle; } \\
\text { must have polyethylene seal cap }\end{array}$ & $\begin{array}{l}\text { Samples can be filtered or unfiltered. Headspace should be } \\
\text { allowed to prevent problems if sample freezes. Seal with } \\
\text { wax or plastic tape. }\end{array}$ \\
\hline LC1199 & $1 \mathrm{~L}$ & $\begin{array}{l}\text { Glass bottle, field-rinsed, Teflon } \\
\text { coated or polyethylene seal cap }\end{array}$ & Contact NWQL. \\
\hline LC1717 & $1 \mathrm{~L}$ & $\begin{array}{l}\text { Glass bottle, narrow neck, field-rinsed; } \\
\text { must have polyethylene seal cap }\end{array}$ & $\begin{array}{l}\text { Preserve with } 1 \mathrm{~mL} / \mathrm{L} \mathrm{HgCl}_{2} \text {; chill and maintain at } \\
4 \mathrm{deg} \mathrm{C} \text {; ship as soon as possible. }\end{array}$ \\
\hline LC1718 & $1 \mathrm{~L}$ & $\begin{array}{l}\text { Glass bottle, narrow neck, field- } \\
\text { rinsed; must have polyethylene } \\
\text { seal cap }\end{array}$ & $\begin{array}{l}\text { Preserve with } 1 \mathrm{~mL} / \mathrm{L} \mathrm{HgCl}_{2} \text {; chill and maintain at } \\
4 \mathrm{deg} \mathrm{C} \text {; ship as soon as possible. }\end{array}$ \\
\hline
\end{tabular}





\section{ANALYTICAL SERVICES}

The Analytical Services section of this catalog is divided into tables 5 through 16 , listing the chemical and biological services offered at the NWQL. Tables 5 through 16, which are summarized as follows, appear at the back of this section. Note: All abbreviations, classifications, and units for these tables are listed in table 2; bottle types are defined in table 4 .

Table 5. Biological determinations listed by laboratory schedule. Determinations are arranged into schedules pertaining to periphyton and phytoplankton in ascending order of schedule number. Chlorophyll a and chlorophyll b cannot be ordered separately.

Table 6. Inorganic determinations for sediment listed alphabetically. Includes those determinations found only in schedules.

Table 7. Inorganic determinations for water listed alphabetically. Includes those determinations found only in schedules.

Table 8. Inorganic determinations for sediment listed by laboratory schedule. Descriptive titles include limitations on the methods.

Table 9. Inorganic determinations for water listed by selected laboratory schedule. Descriptive titles include limitations on the methods.

Table 10. Inorganic determinations for tissue listed by laboratory schedule. Descriptive titles include limitations on the methods.

Table 11. Gross organic determinations listed alphabetically. A list of those organic parameters that can be requested individually. These characteristics also may be combined to form customized District schedules.

Table 12. Organic determinations for sediment listed by laboratory schedule. These schedules may not be broken into smaller schedules, combined with other laboratory codes to form customized District-owned schedules, or added to in any way except by requesting a custom analysis.

Table 13. Organic determinations for water listed by laboratory schedule. These schedules may not be broken into smaller schedules, combined with other laboratory codes into customized District-owned schedules, or added to in any way except by requesting a custom analysis.

Table 14. Organic determinations for tissue listed by laboratory schedule.

Table 15 . Radiochemical determinations listed by laboratory code and schedule. Listed by radiochemical analysis type, and, within each type, determinations are listed numerically by laboratory code or schedule number. Schedule information includes the associated laboratory codes. The types of radiochemical services available are as follows: gamma scans, gross alpha and gross beta, lead, polonium, radium, radon, strontium, thorium, tritium, uranium, carbon-14 dating, and miscellaneous services. 
Table 16. Stable isotope ratios listed by laboratory code and schedule. Ratios may be requested for Carbon-13/Carbon-12, Sulfur-34/Sulfur-32, Deuterium, Oxygen-18/Oxygen-16, and Nitrogen-15/ Nitrogen-14.

NOTE: Shaded laboratory codes in tables 15 and 16 may not be requested individually. They are only available as a unit in the indicated schedules because of the radiochemical techniques used. Unshaded laboratory codes may be requested individually.

\section{$\underline{\text { Requests for Services }}$}

The services listed in this subsection are available to all WRD personnel requiring chemical or biological analyses of samples. However, not all services are available to the same extent or on the same turnaround time. The WRD has established the NWQL to serve the need for chemical and biological analyses. The NWQL has been designed to support the historical level of requests, plus additional expected needs. Each type of service requires equipment, trained analysts, and associated support (log-in, computer services, and warehousing). As a result, the capacity of the Laboratory is limited. Therefore, each analysis type can become overloaded, and other types with little historical precedent for large numbers of requests can be overloaded whenever more than a few requests are received at one time. Also, the Laboratory reflects WRD activity--it is busier at the end of the water year than at other times, so much so that 2.5 times more work arrives in August than in February.

Sometimes projects need detailed work. Additional analytes, low-detection limits, unusual matrices, or perhaps even a new analytical method characterize these needs. The NWQL welcomes these nonroutine requests, but cannot promise as fast a response as that given routine work listed in this catalog. These requests can be handled by "custom" and "special" analyses. There are also occasional requests for priority handling in emergencies.

Moreover, regulatory samples are handled through the Laboratory. The NWQL's main assignment is analysis of samples from hydrologic investigations. This hydrologic work at the Laboratory is mainly at concentration levels far lower than those found during investigations for regulatory purposes. To avoid contamination of the laboratory environment, instrumentation, and staff, many regulatory samples are handled off-site by a contractor. Interested WRD personnel are invited to contact the NWQL to use this additional service.

\section{Selection of Analyses}

Analyses are requested by laboratory codes or schedules. The particular laboratory codes are to be chosen primarily on the basis of the minimum-reporting level desired, but precision should not be overlooked. However, Laboratory personnel might choose another code under several circumstances.

For example, if minimum-reporting levels are comparable, substitution of one laboratory code for another used on more productive equipment might be chosen to favor higher laboratory efficiency. Some codes represent methods that avoid interferences caused by the customer's particular chemical matrix. In other cases, upper ranges of the substituted code may be more in keeping with the customer's matrix. In a few cases, the method requested may have been developed for a higher purity water than the sample proves to be, and more appropriate methods might be substituted. In all cases, the laboratory code actually used will be shown in the results. 
If a procedure with a high detection level is requested and the concentration of the constituent being determined is less than the detection level, a method with a lower detection limit (and usually greater cost) will not be substituted without the customer's specific authorization. The Laboratory will assume responsibility for meeting any constraint implied by the customer's choice of laboratory code or schedule, including the cost of the analysis.

\section{Laboratory Schedules}

A schedule is a collection of laboratory codes (parameters), which are associated because of one of two reasons: (1) convenience to the customer (for example, asking for the major cations schedule is more convenient than asking for the list of all 20 parameters), and (2) laboratory instrumentation (the instrument used to determine the seven Aroclors in SC 1364 is automated to produce only those seven determinations as a unit). Many more schedules exist for inorganic analyses, some created for the laboratory's convenience, others created for programs that are specific to individual Districts. They can be found by perusing the electronic version of the catalog by using the SPN program.

Schedules may be custom-made for individual use. There are restrictions for admissible inclusions of laboratory codes. Schedules should contain laboratory codes of only one type. Present types are as follows:

$\begin{array}{ll}\text { WI } & \text { Water, inorganic } \\ \text { WL } & \text { Water, low-level } \\ \text { WN } & \text { Water, major nutrient } \\ \text { WO } & \text { Water, organic } \\ \text { WR } & \text { Radiochemical or stable isotope ratios } \\ \text { BI } & \text { Bottom material, inorganic } \\ \text { BO } & \text { Bottom material, organic } \\ \text { BR } & \text { Bottom material, radiochemical } \\ \text { BL } & \text { Biological } \\ \text { BG } & \text { Geologic Division } \\ \text { TO } & \text { Tissue, organic } \\ \text { TI } & \text { Tissue, inorganic }\end{array}$

Call the Laboratory or EDOC DENADP for assistance and schedule setup in the NWQL computer systems.

Calculated parameters should not be requested separately unless listed in this Services Catalog, since all calculated values that can be produced from the data (except for those listed in the catalog) are calculated during data retrieval and can be listed in the analytical report.

\section{Provisional Methods}

This catalog of analytical services contains a few new methods developed for the NAWQA program. These methods currently produce provisional data and should be treated as such. These provisional data may or may not be comparable to data produced by other methods. For information concerning data comparability, please contact the Organic Chemistry Program Chief at the NWQL. Provisional 
data are defined as data produced by a method which currently does NOT have a citable reference (TWRI or OFR). Technical memoranda are not citable in reports. Current provisional methods are listed as follows:

Schedule 2001--Pesticides in filtered water, SPE by lab

Schedule 2010--Pesticides in filtered water, SPE by field

Schedule 2050--Pesticides in filtered water, SPE by lab

Schedule 2101--Organochlorine pesticides in biological samples (tissue)

Schedule 2501--Organochlorine pesticides in bed sediments

Schedule 2502--Base/neutral acid extractable compounds in bed sediments

\section{Quality Control and Inorganic Data Review}

Inorganic data review ensures the release of quality data by the NWQL. Once all of the inorganic analyses for a given subsample are completed, they are evaluated by a quality-control (QC) program that resides in the Laboratory Information Management System. The algorithms built into this program check and compare the data.

The following constituents are checked: USEPA Primary Drinking-Water Regulations, cation and anion balance, specific conductance (SPC)/100 (partial cation or anion sum) ratios, residue on evaporation $\left(\mathrm{ROE}\right.$ at $\left.180^{\circ} \mathrm{C}\right) / \mathrm{SPC}$ ratio, $\mathrm{pH}$, less than zero values, and fields with delete codes. The following constituents are compared: onsite and laboratory $\mathrm{pH}$, onsite and laboratory specific conductance, and dissolved and total analyte concentration. If the results of any one of these checks or comparisons, or both, fall outside the prescribed limits for any given analysis, the sample is not approved by the QC program. The results are then passed to the data review chemist with a flag identifying the violation. Subsamples that are approved by the QC program are released to the District.

The data review chemist reviews the unapproved subsamples and makes decisions as mandated by two standard operating procedures (SOP)--the Data Review SOP and the Bottle Mix-up SOP. A historical data base is also used by the data review chemist and the District reanalysis program as an aide to pinpoint questionable analyses.

The Data Review SOP provides a format for evaluating unapproved inorganic samples, samples for reanalysis, and samples that can be released to the District with comments. Comments clarify questions arising from the data. The Data Review SOP also mandates when to refer to the Bottle Mix-up SOP.

The Bottle Mix-up SOP assists the data review chemist in determining if subsample bottles have been mixed-up. If a bottle mix-up is suspected, all bottles of the subsample are physically checked. Routine subsamples are visually checked by the data review chemist. Chain-of-custody subsamples are checked by the bottle warehouse custodian and the data review chemist. When a mix-up is identified, the bottles are relogged in most cases. In cases where information on the bottles does not match information on the Analytical Services Request form, appropriate action is taken to correct discrepancies.

The historical data base is an on-going collection of all the data for each District station. Listed under each station identification (ID) number are all the parameters that were analyzed for that corresponding station ID, the number of analyses for each corresponding parameter, the average of all the values for each parameter, the standard deviation of all the parameter values, and the high and low values of 
each parameter. The District reanalysis program uses the historical data base in the same manner to justify the validity of District reanalysis requests.

In summary, inorganic subsamples that are brought into compliance with the NWQL's QC protocol are released to the District. A thorough effort is made to bring the subsamples that do not comply into agreement with the QC protocol before they are released.

The prescribed limits for evaluating sample analysis are listed as follows:

Constituent

USEPA Primary DrinkingWater Regulations

Cation and anion balance

SPC/100 (cation or anion partial sum)

$\mathrm{ROE}$ at $180^{\circ} \mathrm{C} / \mathrm{SPC}$

$\mathrm{pH}$

Less than 0 values

Fields containing delete codes

Difference between lab and onsite $\mathrm{pH}$

Difference between lab and onsite SPC

Dissolved and total analytes

Filtered and whole water recoverable analytes

\section{Prescribed limit}

Inorganic analysis of all identified drinking-water samples, with the exception of NPDES samples exceeding the USEPA Primary Drinking-Water Regulations, are always reanalyzed (U.S. Environmental Protection Agency, 1993, p. 596).

Refer to Friedman and Erdmann (1982, fig. 15, p. 104).

Must fall between 0.92 and 1.24.

Must fall between 0.55 and 0.86 .

Must fall between 4.00 and 9.00 .

Any negative value will fail the check.

All subsamples with fields containing delete codes fail the check. For further explanation of delete codes, refer to the Laboratory Delete Code list below.

Must be less than $1 \mathrm{pH}$.

This difference must be less than 10 percent.

Dissolved analytes must be less than or equal to the total analytes of the same element or compound

Filtered analytes must be less than or equal to whole water recoverable analytes of the same element or compound. 


\section{Delete Codes}

"A" The samples were analyzed by an alternate method

"B" The sample was broken or spilled in shipment

"D" A delete was requested by the District

"F" An improper filter was used

"I" The required sample type was not received

"M" The results will be sent by a separate memo

"O" There was an insufficient amount of water to complete the analysis

"P" The sample was discarded because of improper preservation

"R" The sample was ruined during analysis

" $U$ " The sample data were unable to be determined because of interference

"W" The sample was warm when it was received. It was therefore discarded

"X" Lab code 586 was reported as 0

"Z" Lab code 588 was reported as 0 
Table 5.--Biological determinations listed by laboratory schedule

\begin{tabular}{|c|c|c|c|c|c|c|c|}
\hline $\begin{array}{l}\text { Schedule } \\
\text { number }\end{array}$ & $\begin{array}{l}\text { Lab. } \\
\text { code }\end{array}$ & $\begin{array}{l}\text { Para- } \\
\text { meter } \\
\text { code }\end{array}$ & MC & Parameter name & $\begin{array}{l}\text { Unit of } \\
\text { measure- } \\
\text { ment }\end{array}$ & $\begin{array}{l}\text { Sample volume } \\
\text { and bottle } \\
\text { designation (see } \\
\text { table 4) }\end{array}$ & MRL \\
\hline \multicolumn{8}{|l|}{ Periphyton } \\
\hline \multirow[t]{3}{*}{671} & & & & Biomass, gravimetric & & 1 filter $\mathrm{CHE}$ & \\
\hline & 611 & 00572 & & Biomass, ash weight & $\mathrm{g} / \mathrm{m}^{2}$ & & 0.001 \\
\hline & 603 & 00573 & & Biomass, dry weight & $\mathrm{g} / \mathrm{m}^{2}$ & & .001 \\
\hline \multirow[t]{3}{*}{1507} & & & & $\begin{array}{l}\text { Chlorophyll, } \\
\text { chromatography } \\
\text { and fluorometry }\end{array}$ & & 1 filter $\mathrm{CHE}$ & \\
\hline & 588 & 70957 & $A$ & Chlorophyll a & $\mathrm{mg} / \mathrm{m}^{2}$ & & .1 \\
\hline & 589 & 70958 & $A$ & Chlorophyll b & $\mathrm{mg} / \mathrm{m}^{2}$ & & .1 \\
\hline \multirow[t]{5}{*}{1708} & & & & $\begin{array}{l}\text { Combined biomass } \\
\text { and chlorophyll }\end{array}$ & & 2 filters $\mathrm{CHE}$ & \\
\hline & 611 & 00572 & $A$ & Biomass, ash weight & $\mathrm{g} / \mathrm{m}^{2}$ & & .001 \\
\hline & 603 & 00573 & $A$ & Biomass, dry weight & $\mathrm{g} / \mathrm{m}^{2}$ & & .001 \\
\hline & 588 & 70957 & A & Chlorophyll a & $\mathrm{mg} / \mathrm{m}^{2}$ & & .1 \\
\hline & 589 & 70958 & A & Chlorophyll b & $\mathrm{mg} / \mathrm{m}^{2}$ & & .1 \\
\hline \multicolumn{8}{|c|}{ Phytoplankton } \\
\hline \multirow[t]{3}{*}{666} & & & & Biomass, gravimetric & & 1 filter $\mathrm{CHY}$ & \\
\hline & 621 & 81353 & $A$ & Biomass, ash weight & $\mathrm{mg} / \mathrm{L}$ & & .1 \\
\hline & 620 & 81354 & A & Biomass, dry weight & $\mathrm{mg} / \mathrm{L}$ & & .1 \\
\hline \multirow[t]{3}{*}{1508} & & & & $\begin{array}{l}\text { Chlorophyll, } \\
\text { chromatography } \\
\text { and fluorometry }\end{array}$ & & 1 filter $\mathrm{CHY}$ & \\
\hline & 586 & 70953 & $A$ & Chlorophyll a & $\mu \mathrm{g} / \mathrm{L}$ & & .1 \\
\hline & 587 & 70954 & A & Chlorophyll b & $\mu \mathrm{g} / \mathrm{L}$ & & .1 \\
\hline \multirow[t]{5}{*}{1509} & & & & $\begin{array}{l}\text { Combined biomass } \\
\text { and chlorophyll }\end{array}$ & & 2 filters $\mathrm{CHY}$ & \\
\hline & 621 & 81353 & $A$ & Biomass, ash weight & $\mathrm{mg} / \mathrm{L}$ & & .1 \\
\hline & 620 & 81354 & A & Biomass, dry weight & $\mathrm{mg} / \mathrm{L}$ & & .1 \\
\hline & 586 & 70953 & A & Chlorophyll a & $\mu \mathrm{g} / \mathrm{L}$ & & .1 \\
\hline & 587 & 70954 & A & Chlorophyll b & $\mu \mathrm{g} / \mathrm{L}$ & & .1 \\
\hline
\end{tabular}



Table 6.--Inorganic determinations for sediment listed alphabetically

\begin{tabular}{|c|c|c|c|c|c|c|c|}
\hline $\begin{array}{l}\text { Lab. } \\
\text { code }\end{array}$ & $\begin{array}{l}\text { Parameter } \\
\text { code }\end{array}$ & Name, phase and method & Units & $\begin{array}{l}\text { Volume } \\
\text { needed }\end{array}$ & $\begin{array}{l}\text { Sample } \\
\text { designa- } \\
\text { tion }^{1}\end{array}$ & MRL & $\begin{array}{c}\text { Call } \\
\text { in } \\
\mathrm{LC}^{2}\end{array}$ \\
\hline 1282 & $01108 \mathrm{C}$ & Aluminum, BTM, DCP & $\mu \mathrm{g} / \mathrm{g}$ & $10 \mathrm{~g}$ & $\mathrm{CU}$ & 10 & 647,1184 \\
\hline 534 & $01098 \mathrm{~A}$ & Antimony, BTM, AA, hydride, & $\mu \mathrm{g} / \mathrm{g}$ & $10 \mathrm{~g}$ & $\mathrm{CU}$ & 1 & 1184 \\
\hline 597 & $01003 \mathrm{C}$ & Arsenic, BTM, AA, hydride & $\mu \mathrm{g} / \mathrm{g}$ & $10 \mathrm{~g}$ & $\mathrm{CU}$ & 1 & 1184 \\
\hline 521 & $01008 \mathrm{~A}$ & Barium, BTM, AA & $\mu \mathrm{g} / \mathrm{g}$ & $10 \mathrm{~g}$ & $\mathrm{CU}$ & 10 & 647,1184 \\
\hline 522 & 01013 A & Beryllium, BTM, AA & $\mu \mathrm{g} / \mathrm{g}$ & $10 \mathrm{~g}$ & $\mathrm{CU}$ & 1 & 647,1184 \\
\hline 1285 & $01023 \mathrm{C}$ & Boron, BTM, DCP & $\mu \mathrm{g} / \mathrm{g}$ & $10 \mathrm{~g}$ & $\mathrm{CU}$ & 10 & 647,1184 \\
\hline 502 & $01028 \mathrm{~B}$ & Cadmium, BTM, AA & $\mu \mathrm{g} / \mathrm{g}$ & $10 \mathrm{~g}$ & $\mathrm{CU}$ & 1 & 647,1184 \\
\hline 696 & 00917 A & Calcium, BTM, AA & $\mathrm{mg} / \mathrm{kg}$ & $10 \mathrm{~g}$ & $\mathrm{CU}$ & 10 & 647,1184 \\
\hline 505 & 01029 B & Chromium, BTM, AA & $\mu \mathrm{g} / \mathrm{g}$ & $10 \mathrm{~g}$ & $\mathrm{CU}$ & 1 & 647,1184 \\
\hline 506 & $01038 \mathrm{~B}$ & Cobalt, BTM, AA & $\mu \mathrm{g} / \mathrm{g}$ & $10 \mathrm{~g}$ & $\mathrm{CU}$ & 5 & 647,1184 \\
\hline 507 & $01043 \mathrm{~B}$ & Copper, BTM, AA & $\mu \mathrm{g} / \mathrm{g}$ & $10 \mathrm{~g}$ & $\mathrm{CU}$ & 1 & 647,1184 \\
\hline 1235 & $00721 \mathrm{~B}$ & Cyanide, BTM, barbituric acid, ASF & $\mathrm{mg} / \mathrm{kg}$ & $10 \mathrm{~g}$ & $\mathrm{CC}$ & .5 & 1242 \\
\hline 1242 & $99480 \mathrm{~A}$ & Digestion for cyanide, BTM & $\cdots$ & $10 \mathrm{~g}$ & $\mathrm{CC}$ & - & \\
\hline 647 & LC0654 A & Digestion for trace metals, BTM & -- & $10 \mathrm{~g}$ & $\mathrm{CU}$ & - & \\
\hline 190 & $01170 \mathrm{~B}$ & Iron, BTM, AA & $\mu \mathrm{g} / \mathrm{g}$ & $10 \mathrm{~g}$ & $\mathrm{CU}$ & 1 & 647,1184 \\
\hline 510 & $01052 \mathrm{~B}$ & Lead, BTM, AA & $\mu \mathrm{g} / \mathrm{g}$ & $10 \mathrm{~g}$ & $\mathrm{CU}$ & 10 & \\
\hline 541 & $01133 \mathrm{~A}$ & Lithium, BTM, AA & $\mu \mathrm{g} / \mathrm{g}$ & $10 \mathrm{~g}$ & $\mathrm{CU}$ & 1 & 647,1184 \\
\hline 697 & $00924 \mathrm{~A}$ & Magnesium, BTM, AA & $\mathrm{mg} / \mathrm{kg}$ & $10 \mathrm{~g}$ & $\mathrm{CU}$ & 10 & 647,1184 \\
\hline 512 & $01053 \mathrm{~A}$ & Manganese, BTM, AA & $\mu \mathrm{g} / \mathrm{g}$ & $10 \mathrm{~g}$ & $\mathrm{CU}$ & .1 & 647,1184 \\
\hline 511 & $71921 \mathrm{~A}$ & Mercury, BTM, CVAA & $\mu \mathrm{g} / \mathrm{g}$ & $10 \mathrm{~g}$ & $\mathrm{CU}$ & .01 & 1184 \\
\hline 523 & $01063 \mathrm{~A}$ & Molybdenum, BTM, AA & $\mu \mathrm{g} / \mathrm{g}$ & $10 \mathrm{~g}$ & $\mathrm{CU}$ & .1 & 647,1184 \\
\hline 524 & $00611 \mathrm{~A}$ & $\begin{array}{l}\mathrm{N} \text {, Ammonia as N, BTM, colorimetric, } \\
\text { salicylate-hypochlorite, ASF }\end{array}$ & $\mathrm{mg} / \mathrm{kg}$ & $10 \mathrm{~g}$ & $\mathrm{CC}$ & .2 & \\
\hline 1211 & $00626 \mathrm{C}$ & $\begin{array}{l}\mathrm{N}, \text { Ammonia plus organic nitrogen as } \mathrm{N} \text {, } \\
\text { BTM, colorimetric, salicylate- } \\
\text { hypochlorite, ASF }\end{array}$ & $\mathrm{mg} / \mathrm{kg}$ & $10 \mathrm{~g}$ & $\mathrm{CC}$ & 20 & \\
\hline 301 & $00608 \mathrm{~B}$ & $\begin{array}{l}\mathrm{N}, \text { Anmmonia as N, DIS, colorimetric, } \\
\text { salicylate-hypochlorite, ASF }\end{array}$ & $\mathrm{mg} / \mathrm{L}$ & $125 \mathrm{~mL}$ & FC & .01 & \\
\hline 513 & $00633 \mathrm{~A}$ & $\begin{array}{l}\mathrm{N}, \text { Nitrite plus nitrate as } \mathrm{N}, \mathrm{BTM} \text {, } \\
\text { cadmium reduction, diazotization, ASF }\end{array}$ & $\mathrm{mg} / \mathrm{kg}$ & $10 \mathrm{~g}$ & $\mathrm{CC}$ & 2 & \\
\hline 519 & $01068 \mathrm{~B}$ & Nickel, BTM, AA & $\mu \mathrm{g} / \mathrm{g}$ & $10 \mathrm{~g}$ & $\mathrm{CU}$ & 10 & 647,1184 \\
\hline 532 & 00339 A & $\begin{array}{l}\text { Oxygen demand, chemical, } \\
\text { BTM, calc. on dry weight }\end{array}$ & nig/kg & $10 \mathrm{~g}$ & $\mathrm{CC}$ & & 904 \\
\hline 515 & $00668 \mathrm{~B}$ & P, Phosphorus as P, BTM, PPMB, ASF & $\mathrm{mg} / \mathrm{kg}$ & $10 \mathrm{~g}$ & $\mathrm{CC}$ & 40 & \\
\hline 698 & 00938 A & Potassium, BTM, AA & $\mathrm{mg} / \mathrm{kg}$ & $10 \mathrm{~g}$ & $\mathrm{CU}$ & 10 & 647 \\
\hline 1184 & $01184 \mathrm{~A}$ & Preparation for BTM & - & $10 \mathrm{~g}$ & $\mathrm{CU}$ & & \\
\hline
\end{tabular}


Table 6.--Inorganic determinations for sediment listed alphabetically--Continued

\begin{tabular}{|c|c|c|c|c|c|c|c|}
\hline $\begin{array}{l}\text { Lab. } \\
\text { code }\end{array}$ & $\begin{array}{l}\text { Parameter } \\
\text { code }\end{array}$ & Name, phase and method & Units & $\begin{array}{l}\text { Volume } \\
\text { needed }\end{array}$ & $\begin{array}{c}\text { Sample } \\
\text { designa- } \\
\text { tion }^{1}\end{array}$ & MRL & $\begin{array}{c}\text { Call } \\
\text { in } \\
L^{2}\end{array}$ \\
\hline$\overline{517}$ & $01148 \mathrm{~A}$ & Selenium, BTM, hydride & $\overline{\mu g / g}$ & $\overline{10 \mathrm{~g}}$ & $\overline{\mathrm{CU}}$ & $\overline{1}$ & $\overline{1184}$ \\
\hline 699 & $00934 \mathrm{~A}$ & Sodium, BTM, AA & mg/kg & $10 \mathrm{~g}$ & $\mathrm{CU}$ & 10 & 647 \\
\hline 516 & 00496 A & Solids, VOI, BTM, GR & $\mathrm{mg} / \mathrm{kg}$ & $10 \mathrm{~g}$ & $\mathrm{CC}$ & 1 & 647 \\
\hline 530 & $01083 \mathrm{~A}$ & Strontium, BTM, AA & $\mu g / g$ & $10 \mathrm{~g}$ & $\mathrm{CU}$ & 1 & 647,1184 \\
\hline 518 & $01093 \mathrm{~A}$ & Zinc, BTM, AA & $\mu g / g$ & $10 \mathrm{~g}$ & $\mathrm{CU}$ & 1 & 647,1184 \\
\hline
\end{tabular}

${ }^{1}$ See table 4.

${ }^{2}$ LC 647 (bottom material digestion) may be called in automatically, in addition to lab code 1184 , for those bottom material analyses requiring a specific digestion prior to metals analysis.

LC 1184 (bottom material preparation) is required prior to any analyses requested for bottom material samples. It is called in automatically by those analyses. Only one bottom material preparation charge will be added since all bottom materials go through the same preparation step. 
Table 7.--Inorganic determinations for water listed alphabetically

\begin{tabular}{|c|c|c|c|c|c|c|c|}
\hline $\begin{array}{l}\text { Lab. } \\
\text { code }\end{array}$ & $\begin{array}{c}\text { Parameter } \\
\text { code }\end{array}$ & Name, phase, and method & Units & $\begin{array}{r}\text { Volume } \\
\text { needed } \\
(\mathrm{mL})\end{array}$ & $\begin{array}{c}\text { Sample } \\
\text { designa- } \\
\text { tion }^{1}\end{array}$ & MRL & $\begin{array}{l}\text { Call } \\
\text { in } \\
\mathrm{LC}^{2}\end{array}$ \\
\hline 1266 & $71825 \mathrm{~B}$ & $\begin{array}{l}\text { Acidity as } \mathrm{H} \text {, lab., LIS, WWR, } \\
\text { ET, 2nd derivative }\end{array}$ & $\mathrm{mg} / \mathrm{L}$ & 75 & RU & 0.01 & \\
\hline 1 & $71825 \mathrm{~A}$ & Acidity as $\mathrm{H}$, lab., WWR, ET & $\mathrm{mg} / \mathrm{L}$ & 50 & RU & 1 & \\
\hline 1270 & $90410 \mathrm{~B}$ & $\begin{array}{l}\text { Alkalinity as } \mathrm{CaCO}_{3} \text {, lab., LIS, WWR, } \\
\mathrm{ET}, \text { 2nd derivative }\end{array}$ & $\mathrm{mg} / \mathrm{L}$ & 75 & RU & .5 & \\
\hline 70 & $90410 \mathrm{~A}$ & Alkalinity as $\mathrm{CaCO}_{3}$, lab., WWR, ET & $\mathrm{mg} / \mathrm{L}$ & 100 & RU & 1 & \\
\hline 1284 & $01106 \mathrm{E}$ & Aluminum, DIS, DCP & $\mu \mathrm{g} / \mathrm{L}$ & 100 & FA & 10 & \\
\hline 1283 & $01105 \mathrm{C}$ & Aluminum, WWR, DCP & $\mu \mathrm{g} / \mathrm{L}$ & 100 & RA & 10 & 1735 \\
\hline 77 & $01095 \mathrm{~A}$ & Antimony, DIS, AA, hydride & $\mu g / L$ & 50 & FA & 1 & \\
\hline 80 & $01097 \mathrm{~A}$ & Antimony, WWR, AA, hydride & $\mu \mathrm{g} / \mathrm{L}$ & 50 & RAH & 1 & \\
\hline 112 & $01000 \mathrm{~B}$ & Arsenic, DIS, AA, hydride & $\mu \mathrm{g} / \mathrm{L}$ & 50 & FA & 1 & \\
\hline 118 & $01002 \mathrm{~B}$ & Arsenic, WWR, AA, hydride & $\mu \mathrm{g} / \mathrm{L}$ & 50 & RAH & 1 & \\
\hline 1584 & $01002 \mathrm{C}$ & $\begin{array}{l}\text { Arsenic, WWR, GFAA, USEPA } \\
\text { (drinking water only) }\end{array}$ & $\mu \mathrm{g} / \mathrm{L}$ & 250 & RAH & 1 & 1586 \\
\hline 7 & $01005 \mathrm{~B}$ & Barium, DIS, AA & $\mu \mathrm{g} / \mathrm{L}$ & 50 & FA & 100 & \\
\hline 641 & $01005 \mathrm{C}$ & Barium, DIS, ICP & $\mu \mathrm{g} / \mathrm{L}$ & 100 & FA & 1 & \\
\hline 234 & $01007 \mathrm{~A}$ & Barium, WWR, AA & $\mu \mathrm{g} / \mathrm{L}$ & 50 & RA & 100 & 1735 \\
\hline 170 & $01010 \mathrm{~A}$ & Beryllium, DIS, AA & $\mu \mathrm{g} / \mathrm{L}$ & 25 & FA & 10 & \\
\hline 655 & $01010 \mathrm{~B}$ & Beryllium, DIS, ICP & $\mu g /$ & 100 & FA & .5 & \\
\hline 236 & $01012 \mathrm{~A}$ & Beryllium, WWR, AA & $\mu \mathrm{g} / \mathrm{L}$ & 50 & RA & 10 & 1735 \\
\hline 1183 & $01020 \mathrm{~B}$ & Boron, DIS, DCP & $\mu \mathrm{g} / \mathrm{L}$ & 50 & FU & 10 & \\
\hline 1286 & $01022 \mathrm{~B}$ & Boron, WWR, DCP & $\mu \mathrm{g} / \mathrm{L}$ & 250 & RA & 10 & 1735 \\
\hline 1246 & $71870 \mathrm{E}$ & Bromide, DIS, fluorescein, ASF & $\mathrm{mg} / \mathrm{L}$ & 250 & FU & .01 & \\
\hline 1258 & $71870 \mathrm{~F}$ & Bromide, LIS, DIS, IC & $\mathrm{mg} / \mathrm{L}$ & 50 & $\mathrm{FU}$ & .01 & \\
\hline 126 & $01025 \mathrm{~A}$ & Cadmium, DIS, AA & $\mu \mathrm{g} / \mathrm{L}$ & 25 & FA & 10 & \\
\hline 1554 & $01025 \mathrm{~F}$ & Cadmium, DIS, GFAA & $\mu \mathrm{g} / \mathrm{L}$ & 50 & FA & 1 & \\
\hline 673 & $01025 \mathrm{D}$ & Cadmium, DIS, ICP & $\mu \mathrm{g} / \mathrm{L}$ & 100 & FA & 1 & \\
\hline 1250 & $01025 \mathrm{E}$ & Cadmium, LL, DIS, GFAA & $\mu \mathrm{g} / \mathrm{L}$ & 250 & FAB & .1 & \\
\hline 131 & $01027 \mathrm{~A}$ & Cadmium, WWR, AA & $\mu \mathrm{g} / \mathrm{L}$ & 50 & RA & 10 & 1735 \\
\hline 1555 & $01027 \mathrm{~F}$ & Cadmium, WWR, GFAA & $\mu \mathrm{g} / \mathrm{L}$ & 50 & RA & 1 & 1735 \\
\hline 12 & $0915 \mathrm{C}$ & Calcium, DIS, AA & $\mathrm{mg} / \mathrm{L}$ & 50 & FA & .1 & \\
\hline 659 & $0915 \mathrm{D}$ & Calcium, DIS, ICP & $\mathrm{mg} / \mathrm{L}$ & 100 & FA & .02 & \\
\hline 831 & $0915 \mathrm{~B}$ & Calcium, LIS, DIS, AA & $\mathrm{mg} / \mathrm{L}$ & 50 & FA & .01 & \\
\hline 1273 & $0915 \mathrm{E}$ & Calcium, LIS, DIS, ICP & $\mathrm{mg} / \mathrm{L}$ & 100 & FA & .02 & \\
\hline 324 & $0916 \mathrm{~A}$ & Calcium, WWR, AA, USEPA & $\mathrm{mg} / \mathrm{L}$ & 50 & RAE & 1 & 124 \\
\hline 244 & $0916 \mathrm{~B}$ & Calcium, WWR, AA, USGS & $\mathrm{mg} / \mathrm{L}$ & 50 & RA & 1 & 1735 \\
\hline 1571 & $0940 \mathrm{~J}$ & Chloride, DIS, IC & $\mathrm{mg} / \mathrm{L}$ & 50 & FU & 1 & \\
\hline 1259 & 0940 I & Chloride, LIS, DIS, IC & $\mathrm{mg} / \mathrm{L}$ & 50 & FU & .01 & \\
\hline
\end{tabular}


Table 7.--Inorganic determinations for water listed alphabetically--Continued

\begin{tabular}{|c|c|c|c|c|c|c|c|}
\hline $\begin{array}{l}\text { Lab. } \\
\text { code }\end{array}$ & $\begin{array}{l}\text { Parameter } \\
\text { code }\end{array}$ & Name, phase, and method & Units & $\begin{array}{r}\text { Volume } \\
\text { needed } \\
(\mathrm{mL})\end{array}$ & $\begin{array}{c}\text { Sample } \\
\text { designa- } \\
\text { tion }{ }^{1}\end{array}$ & MRL & $\begin{array}{l}\text { Call } \\
\text { in } \\
\mathrm{LC}^{2}\end{array}$ \\
\hline 722 & $01030 \mathrm{E}$ & Chromium, DIS, ICP & $\mu \mathrm{g} / \mathrm{L}$ & 100 & FA & 5 & \\
\hline 16 & $01032 \mathrm{~A}$ & Chromium, hexavalent, DIS, chel., AA & $\mu \mathrm{g} / \mathrm{L}$ & 250 & FA & 1 & \\
\hline 1251 & $01030 \mathrm{D}$ & Chromium, LL, DIS, GFAA & $\mu \mathrm{g} / \mathrm{L}$ & 250 & FAB & .5 & \\
\hline 1936 & $01030 \mathrm{I}$ & Chromium, DIS, GFAA & $\mu \mathrm{g} / \mathrm{L}$ & 50 & FA & 1 & \\
\hline 1937 & $01034 \mathrm{E}$ & Chromium, WWR, GFAA & $\mu \mathrm{g} / \mathrm{L}$ & 50 & RA & 1 & 1735 \\
\hline 148 & $01035 \mathrm{~A}$ & Cobalt, DIS, AA & $\mu \mathrm{g} / \mathrm{L}$ & 25 & FA & 50 & \\
\hline 1556 & $01035 \mathrm{~F}$ & Cobalt, DIS, GFAA & $\mu g / L$ & 50 & FA & 1 & \\
\hline 644 & $01035 \mathrm{C}$ & Cobalt, DIS, ICP & $\mu \mathrm{g} / \mathrm{L}$ & 100 & FA & 3 & \\
\hline 1252 & $01035 \mathrm{E}$ & Cobalt, LL, DIS, GFAA & $\mu \mathrm{g} / \mathrm{L}$ & 250 & FAB & .5 & \\
\hline 149 & 01037 A & Cobalt, WWR, AA & $\mu g / L$ & 50 & RA & 50 & 1735 \\
\hline 1557 & $01037 \mathrm{~F}$ & Cobalt, WWR, GFAA & $\mu g / L$ & 50 & RA & 1 & 1735 \\
\hline 20 & $00080 \mathrm{~A}$ & Color reported in Pt-Co units & Units & 100 & $\mathrm{RCB}$ & 1 & \\
\hline 151 & $01040 \mathrm{~A}$ & Copper, DIS,AA & $\mu \mathrm{g} / \mathrm{L}$ & 25 & FA & 10 & \\
\hline 1558 & $01040 \mathrm{~F}$ & Copper, DIS, GFAA & $\mu \mathrm{g} / \mathrm{L}$ & 50 & FA & 1 & \\
\hline 657 & $01040 \mathrm{C}$ & Copper, DIS, ICP & $\mu g / L$ & 100 & FA & 10 & \\
\hline 1253 & $01040 \mathrm{E}$ & Copper, LL, DIS, GFAA & $\mu \mathrm{g} / \mathrm{L}$ & 250 & FAB & .5 & \\
\hline 156 & 01042 A & Copper, WWR, AA & $\mu \mathrm{g} / \mathrm{L}$ & 50 & RA & 10 & 1735 \\
\hline 1559 & $01042 \mathrm{~F}$ & Copper, WWR, GFAA & $\mu \mathrm{g} / \mathrm{L}$ & 50 & RA & 1 & 1735 \\
\hline 880 & $00723 \mathrm{~A}$ & Cyanide, DIS, barbituric acid, ASF & $\mathbf{m g} / \mathrm{L}$ & 100 & LCO880 & .01 & \\
\hline 23 & $00720 \mathrm{~A}$ & Cyanide, WWR, barbituric acid, ASF & $\mathrm{mg} / \mathrm{L}$ & 50 & LCO023 & .01 & \\
\hline 24 & $71820 \mathrm{~A}$ & Density@20 degrees C, filtered, GR & $\mathrm{g} / \mathrm{mL}$ & 100 & FU & .99 & \\
\hline 1586 & $\cdots \mathbf{B}$ & Digestion for As and Se, USEPA & - & 250 & RAH & - & \\
\hline 124 & 99447 A & Digestion for trace metals, USEPA & $\cdots$ & 250 & RAE & -- & \\
\hline 1735 & $99870 \mathrm{~B}$ & Digestion for trace metals, USGS & $\cdots$ & 250 & RA & -- & \\
\hline 31 & $00950 \mathrm{~B}$ & Fluoride, DIS, ISE & $\mathrm{mg} / \mathrm{L}$ & 50 & FU & .1 & \\
\hline 1260 & $00950 \mathrm{D}$ & Fluoride, LIS, DIS, IC & $\mathrm{mg} / \mathrm{L}$ & 50 & FU & .01 & \\
\hline 1202 & $71865 \mathrm{D}$ & $\begin{array}{l}\text { Iodide, DIS, ceric-arsenious } \\
\text { oxidation, ASF }\end{array}$ & $\mathrm{mg} / \mathrm{L}$ & 50 & FU & .001 & \\
\hline 172 & $01046 \mathrm{C}$ & Iron, DIS, AA & $\mu \mathrm{g} / \mathrm{L}$ & 25 & FA & 10 & \\
\hline 645 & $01046 \mathrm{D}$ & Iron, DIS, ICP & $\mu \mathrm{g} / \mathrm{L}$ & 100 & FA & 3 & \\
\hline 1271 & $01046 \mathrm{E}$ & Iron, LIS, DIS, ICP & $\mu \mathrm{g} / \mathrm{L}$ & 50 & FA & 3 & \\
\hline 189 & $01045 \mathrm{~B}$ & Iron, WWR, AA & $\mu g / L$ & 50 & RA & 10 & 1735 \\
\hline 191 & 01049 A & Lead, DIS, AA & $\mu \mathrm{g} / \mathrm{L}$ & 25 & FA & 100 & \\
\hline 1560 & $01049 \mathrm{~F}$ & Lead, DIS, GFAA & $\mu \mathrm{g} / \mathrm{L}$ & 50 & $\mathrm{FA}$ & 1 & \\
\hline 646 & $01049 \mathrm{C}$ & Lead, DIS, ICP & $\mu \mathrm{g} / \mathrm{L}$ & 100 & FA & 10 & \\
\hline 1254 & $01049 \mathrm{E}$ & Lead, LL, DIS, GFAA & $\mu \mathrm{g} / \mathrm{L}$ & 250 & FAB & .5 & \\
\hline 192 & $01051 \mathrm{~A}$ & Lead, WWR, AA & $\mu \mathrm{g} / \mathrm{L}$ & 50 & RA & 100 & 1735 \\
\hline 1561 & $01051 \mathrm{~F}$ & Lead, WWR, GFAA & $\mu \mathrm{g} / \mathrm{L}$ & 50 & RA & 1 & 1735 \\
\hline 39 & $01130 \mathrm{~A}$ & Lithium, DIS, AA & $\mu g / L$ & 25 & FA & 10 & \\
\hline 664 & $01130 \mathrm{~B}$ & Lithium, DIS, ICP & $\mu g / L$ & 100 & FA & 4 & \\
\hline
\end{tabular}


Table 7.--Inorganic determinations for water listed alphabetically--Continued

\begin{tabular}{|c|c|c|c|c|c|c|c|}
\hline $\begin{array}{l}\text { Lab. } \\
\text { code }\end{array}$ & $\begin{array}{l}\text { Parameter } \\
\text { code }\end{array}$ & Name, phase, and method & Units & $\begin{array}{r}\text { Volume } \\
\text { needed } \\
(\mathrm{mL})\end{array}$ & $\begin{array}{l}\text { Sample } \\
\text { designa- } \\
\text { tion }^{1}\end{array}$ & MRL & $\begin{array}{l}\text { Call } \\
\text { in } \\
\mathrm{LC}^{2}\end{array}$ \\
\hline 277 & $01132 \mathrm{~A}$ & Lithium, WWR, AA & $\mu \mathrm{g} / \mathrm{L}$ & 50 & RA & 10 & 1735 \\
\hline 40 & 00925 B & Magnesium, DIS, AA & $\mathrm{mg} / \mathrm{L}$ & 50 & FA & .1 & \\
\hline 663 & $00925 \mathrm{C}$ & Magnesium, DIS, ICP & $\mathrm{mg} / \mathrm{L}$ & 100 & FA & .01 & \\
\hline 832 & $00925 \mathrm{~A}$ & Magnesium, LIS, DIS, AA & $\mathrm{mg} / \mathrm{L}$ & 50 & FA & .01 & \\
\hline 1274 & $00925 \mathrm{D}$ & Magnesium, LIS, DIS, ICP & $\mathrm{mg} / \mathrm{L}$ & 100 & FA & .01 & \\
\hline 325 & 00927 A & Magnesium, WWR, USEPA, AA & $\mathrm{mg} / \mathrm{L}$ & 50 & RAE & .1 & 124 \\
\hline 261 & $00927 \mathrm{~B}$ & Magnesium, WWR, USGS, AA & $\mathrm{mg} / \mathrm{L}$ & 50 & RA & .1 & 1735 \\
\hline 42 & $01056 \mathrm{~A}$ & Manganese, DIS, AA & $\mu g / L$ & 25 & FA & 10 & \\
\hline 648 & $01056 \mathrm{C}$ & Manganese, DIS, ICP & $\mu \mathrm{g} / \mathrm{L}$ & 100 & FA & 1 & \\
\hline 1272 & $01056 \mathrm{E}$ & Manganese, LIS,DIS, ICP & $\mu g / L$ & 100 & FA & 1 & \\
\hline 1255 & $01056 \mathrm{D}$ & Manganese, LL,DIS, GFAA & $\mu \mathrm{g} / \mathrm{L}$ & 250 & FAB & .2 & \\
\hline 41 & $01055 \mathrm{~A}$ & Manganese, WWR, AA & $\mu \mathrm{g} / \mathrm{L}$ & 50 & RA & 10 & 1735 \\
\hline 226 & $71890 \mathrm{~B}$ & Mercury, DIS, CVAA, auto. & $\mu \mathrm{g} / \mathrm{L}$ & 200 & FAM & .1 & \\
\hline 227 & $71900 \mathrm{~B}$ & Mercury, WWR, CVAA & $\mu g / L$ & 200 & RAM & .1 & \\
\hline 904 & 00495 B & Moisture content by weight & $\%$ & $1 \mathrm{~g}$ & $\mathrm{CU}$ & & \\
\hline 649 & $01060 \mathrm{~A}$ & Molybdenum, DIS, ICP & $\mu g / L$ & 100 & FA & 10 & \\
\hline 110 & $01060 \mathrm{~B}$ & Molybdenum, DIS, chel., AA & $\mu \mathrm{g} / \mathrm{L}$ & 250 & FA & 1 & \\
\hline 265 & 01062 A & Molybdenum, WWR, AA & $\mu \mathrm{g} / \mathrm{L}$ & 250 & RA & 1 & 1735 \\
\hline 830 & 00608 A & $\begin{array}{l}\mathrm{N} \text {, Ammonia as N, LL, DIS, colorimetric, } \\
\text { salicylate-hypochlorite, ASF }\end{array}$ & $\mathrm{mg} / \mathrm{L}$ & 125 & FC & .002 & \\
\hline 1278 & $00608 \mathrm{C}$ & $\begin{array}{l}\mathrm{N}, \text { Ammonia as N, LL, DIS, colorimetric, } \\
\text { salicylate-hypochlorite, ASF, unpreserved }\end{array}$ & $\mathrm{mg} / \mathrm{L}$ & 20 & FU & .002 & \\
\hline 1687 & $00623 \mathrm{C}$ & $\begin{array}{l}\mathrm{N}, \text { Ammonia plus organicnitrogen as } \mathrm{N} \text {, } \\
\text { DIS, colorimetric, salicylate- } \\
\text { hypochlorite, ASF }\end{array}$ & $\mathrm{mg} / \mathrm{L}$ & 125 & FC & .2 & \\
\hline 1688 & $00625 \mathrm{C}$ & $\begin{array}{l}\mathrm{N}, \text { Ammonia plus organic nitrogen as } \mathrm{N} \text {, } \\
\mathrm{WWR} \text {, colorimetric, salicylate- } \\
\text { hypochlorite, ASF }\end{array}$ & $\mathrm{mg} / \mathrm{L}$ & 125 & RC & .2 & \\
\hline 1261 & $00618 \mathrm{D}$ & $\mathrm{N}$, Nitrate as N, LL, DIS, IC & $\mathrm{mg} / \mathrm{L}$ & 250 & FU & .01 & \\
\hline 160 & $00613 \mathrm{~B}$ & $\mathrm{~N}$, Nitrite as N, DIS, diazotization, ASF & $\mathrm{mg} / \mathrm{L}$ & 125 & FC & .01 & \\
\hline 827 & 00613 A & $\begin{array}{l}\mathrm{N}, \text { Nitrite as } \mathrm{N}, \mathrm{LL}, \mathrm{DIS} \text {, diazotization, } \\
\text { ASF }\end{array}$ & $\mathrm{mg} / \mathrm{L}$ & 125 & FC & .001 & \\
\hline 1578 & $00631 \mathrm{C}$ & $\begin{array}{l}\mathrm{N}, \text { Nitrite plus Nitrate as } \mathrm{N}, \mathrm{DIS} \text {, } \\
\text { cadmium reduction, diazotization, } \\
\text { ASF, unpreserved }\end{array}$ & $\mathrm{mg} / \mathrm{L}$ & 25 & FU & .05 & \\
\hline 228 & $00631 \mathrm{~B}$ & $\begin{array}{l}\mathrm{N}, \text { Nitrite plus Nitrate as } \mathrm{N}, \mathrm{DIS}, \\
\text { cadmium reduction, diazotization, ASF }\end{array}$ & $\mathrm{mg} / \mathrm{L}$ & 125 & FC & .05 & \\
\hline 826 & 00631 A & $\begin{array}{l}\mathrm{N}, \text { Nitrite plus Nitrate as } \mathrm{N}, \mathrm{LL}, \mathrm{DIS} \text {, } \\
\text { cadmium reduction, diazotization, ASF }\end{array}$ & $\mathrm{mg} / \mathrm{L}$ & 125 & $\mathrm{FC}$ & .005 & \\
\hline 1570 & $00602 \mathrm{~B}$ & $\mathrm{~N}$, Total nitrogen as $\mathrm{N}, \mathrm{DIS}$, Antek & $\mathrm{mg} / \mathrm{L}$ & 50 & FC & .1 & \\
\hline 197 & $01065 \mathrm{~A}$ & Nickel, DIS, AA & $\mu \mathrm{g} / \mathrm{L}$ & 25 & FA & 100 & \\
\hline 1562 & $01065 \mathrm{~F}$ & Nickel, DIS, GFAA & $\mu \mathrm{g} / \mathrm{L}$ & 50 & FA & 1 & \\
\hline
\end{tabular}


Table 7.--Inorganic determinations for water listed alphabetically--Continued

\begin{tabular}{|c|c|c|c|c|c|c|c|}
\hline $\begin{array}{l}\text { Lab. } \\
\text { code }\end{array}$ & $\begin{array}{l}\text { Parameter } \\
\text { code }\end{array}$ & Name, phase, and method & Units & $\begin{array}{r}\text { Volume } \\
\text { needed } \\
(\mathrm{mL})\end{array}$ & $\begin{array}{c}\text { Sample } \\
\text { designa- } \\
\text { tion }^{1}\end{array}$ & MRI & $\begin{array}{l}\text { Call } \\
\text { in } \\
\mathrm{LC}^{2}\end{array}$ \\
\hline 721 & $01065 \mathrm{E}$ & Nickel, DIS, ICP & $\mu \mathrm{g} / \mathrm{L}$ & 100 & FA & 10 & \\
\hline 1256 & $01065 \mathrm{D}$ & Nickel, LL, DIS, GFAA & $\mu \mathrm{g} / \mathrm{L}$ & 250 & FAB & 1 & \\
\hline 1563 & $01067 \mathrm{~F}$ & Nickel, WWR, GFAA & $\mu \mathrm{g} / \mathrm{L}$ & 50 & RA & 1 & 1735 \\
\hline 198 & $01067 \mathrm{~A}$ & Nickel, WWR, AA & $\mu \mathrm{g} / \mathrm{L}$ & 50 & RA & 100 & 1735 \\
\hline 76 & 00340 B & Oxygen demand, chemical, water & $\mathrm{mg} / \mathrm{L}$ & 100 & LC0076 & 10 & \\
\hline 162 & $00671 \mathrm{~B}$ & $\begin{array}{l}\text { P, Phosphate as P, ortho, } \\
\text { DIS, PPMB, ASF }\end{array}$ & $\mathrm{mg} / \mathrm{L}$ & 125 & FC & .01 & \\
\hline 1262 & $00671 \mathrm{G}$ & $\mathrm{P}$, Phosphate as $\mathrm{P}$, ortho, LIS, IC & $\mathrm{mg} / \mathrm{L}$ & 50 & FU & .01 & \\
\hline 828 & 00671 A & $\begin{array}{l}\text { P, Phosphate as P, ortho, LL, } \\
\text { DIS, PPMB, ASF }\end{array}$ & $\mathrm{mg} / \mathrm{L}$ & 125 & FC & .001 & \\
\hline 1277 & $00671 \mathrm{D}$ & $\begin{array}{l}\text { P, Phosphate as } \mathrm{P} \text {, ortho, } \mathrm{LL} \text {, } \\
\text { DIS, PPMB, ASF, unpreserved }\end{array}$ & $\mathrm{mg} / \mathrm{L}$ & 20 & FU & .001 & \\
\hline 279 & 00677 A & $\begin{array}{l}\text { P, Phosphate, ortho plus } \\
\text { hydrolizable as P, DIS, PPMB, ASF }\end{array}$ & $\mathrm{mg} / \mathrm{L}$ & 125 & FC & .01 & \\
\hline 282 & $00678 \mathrm{~A}$ & $\begin{array}{l}\text { P, Phosphate, ortho plus } \\
\text { hydrolizable as WWR, PPMB, ASF }\end{array}$ & $\mathrm{mg} / \mathrm{L}$ & 125 & RC & .01 & \\
\hline 1685 & $00666 \mathrm{C}$ & P, Phosphorus as P, DIS, PPMB, ASF & $\mathrm{mg} / \mathrm{L}$ & 125 & FC & .01 & \\
\hline 829 & 00666 A & P, Phosphorus as P, LL, DIS, PPMB, ASF & $\mathrm{mg} / \mathrm{L}$ & 125 & FC & .001 & \\
\hline 837 & 00665 A & $\mathrm{P}, \mathrm{Phosphorus}$ as $\mathrm{P}, \mathrm{LL}, \mathrm{WWR}, \mathrm{PPMB}, \mathrm{ASF}$ & $\mathrm{mg} / \mathrm{L}$ & 125 & RC & .001 & \\
\hline 1686 & $00665 \mathrm{C}$ & P, Phosphorus as P, WWR, PPMB, ASF & $\mathrm{mg} / \mathrm{L}$ & 125 & RC & .01 & \\
\hline 68 & $00403 \mathrm{~A}$ & pH, lab., elec. & Units & 50 & RU & .1 & \\
\hline 1268 & 00403 B & pH, lab., LIS, elec. & Units & 75 & RU & .1 & \\
\hline 54 & 00935 B & Potassium, DIS, AA & $\mathrm{mg} / \mathrm{L}$ & 50 & FA & .1 & \\
\hline 833 & $00935 \mathrm{~A}$ & Potassium, LL, DIS, AA & $\mathrm{mg} / \mathrm{L}$ & 50 & FA & .01 & \\
\hline 327 & 00937 A & Potassium, WWR, USEPA, AA & $\mathrm{mg} / \mathrm{L}$ & 50 & RAE & .1 & 124 \\
\hline 321 & 00937 B & Potassium, WWR, USGS, AA & $\mathrm{mg} / \mathrm{L}$ & 50 & RA & .1 & 1735 \\
\hline 87 & $01145 \mathrm{~A}$ & Selenium, DIS, hydride, auto. & $\mu \mathrm{g} / \mathrm{L}$ & 50 & FA & 1 & \\
\hline 1585 & $01147 \mathrm{~B}$ & $\begin{array}{l}\text { Selenium, WWR, GFAA, USEPA } \\
\text { (drinking water only) }\end{array}$ & $\mu g / L$ & 250 & RAH & 1 & 1586 \\
\hline 286 & $01147 \mathrm{~A}$ & Selenium, WWR, hydride, auto. & $\mu g / L$ & 50 & RAH & 1 & \\
\hline 667 & $00955 \mathrm{D}$ & Silica as $\mathrm{SiO}_{2}$, DIS, ICP & $\mathrm{mg} / \mathrm{L}$ & 100 & FA & .01 & \\
\hline 56 & $00955 \mathrm{C}$ & $\begin{array}{l}\text { Silica as } \mathrm{SiO}_{2}, \mathrm{DIS} \text {, molybdate } \\
\text { blue, } \mathrm{ASF}\end{array}$ & $\mathrm{mg} / \mathrm{L}$ & 25 & FU & .1 & \\
\hline 1275 & $00955 \mathrm{E}$ & Silica as $\mathrm{SiO}_{2}$, LIS, DIS, ICP & $\mathrm{mg} / \mathrm{L}$ & 100 & FA & .01 & \\
\hline 1552 & $01075 \mathrm{~F}$ & Silver, DIS, GFAA & $\mu g / L$ & 50 & FA & 1 & \\
\hline 723 & $01075 \mathrm{C}$ & Silver, DIS, ICP & $\mu g / L$ & 100 & FA & 1 & \\
\hline 1553 & $01077 \mathrm{~F}$ & Silver, WWR, GFAA & $\mu g / L$ & 50 & RA & 1 & 1735 \\
\hline 1276 & $00930 \mathrm{D}$ & Sodium, DIS, LIS, ICP & $\mathrm{mg} / \mathrm{L}$ & 100 & FA & .2 & \\
\hline 59 & $00930 \mathrm{~B}$ & Sodium, DIS, AA & $\mathrm{mg} / \mathrm{L}$ & so & FA & .1 & \\
\hline 675 & $00930 \mathrm{C}$ & Sodium, DIS, ICP & $\mathrm{mg} / \mathrm{L}$ & 100 & FA & .2 & \\
\hline
\end{tabular}


Table 7.--Inorganic determinations for water listed alphabetically--Continued

\begin{tabular}{|c|c|c|c|c|c|c|c|}
\hline $\begin{array}{l}\text { Lab. } \\
\text { code }\end{array}$ & $\begin{array}{l}\text { Parameter } \\
\text { code }\end{array}$ & Name, phase, and method & Units & $\begin{array}{r}\text { Volume } \\
\text { needed } \\
(\mathrm{mL})\end{array}$ & $\begin{array}{c}\text { Sample } \\
\text { designa- } \\
\text { tion }^{1}\end{array}$ & MRL & $\begin{array}{l}\text { Call } \\
\text { in } \\
\text { LC }^{2}\end{array}$ \\
\hline 834 & $00930 \mathrm{~A}$ & Sodium, LIS, DIS, AA & $\mathrm{mg} / \mathrm{L}$ & 50 & FA & 0.01 & \\
\hline 326 & 00929 A & Sodium, WWR, AA, USEPA & $\mathrm{mg} / \mathrm{L}$ & 50 & RAE & .1 & 124 \\
\hline 320 & $00929 \mathrm{~B}$ & Sodium, WWR, AA, USGS & $\mathrm{mg} / \mathrm{L}$ & 50 & RA & .1 & 1735 \\
\hline 159 & $00515 \mathrm{~B}$ & Solids, ROE@105 deg C, DIS, GR & $\mathrm{mg} / \mathrm{L}$ & 250 & FU & 1 & \\
\hline 169 & $00530 \mathrm{~B}$ & Solids, ROE@105 deg C, SUS, GR & $\mathrm{mg} / \mathrm{L}$ & 250 & LCO169 & 1 & \\
\hline 165 & $00500 \mathrm{~A}$ & Solids, ROE@105 deg C, total, GR & $\mathrm{mg} / \mathrm{L}$ & 250 & RU & 1 & \\
\hline 27 & $70300 \mathrm{~A}$ & Solids, ROE@180 deg C, DIS, GR & $\mathrm{mg} / \mathrm{L}$ & 500 & FU & 1 & \\
\hline 229 & $00520 \mathrm{~A}$ & Solids, VOI, DIS, GR & $\mathrm{mg} / \mathrm{L}$ & 250 & FU & 1 & \\
\hline 49 & 00535 A & Solids, VOI, SUS, GR & $\mathrm{mg} / \mathrm{L}$ & 500 & LC0169 & 1 & \\
\hline 85 & 00505 A & Solids, VOI, total, GR & $\mathrm{mg} / \mathrm{L}$ & 250 & RU & 1 & \\
\hline 69 & $90095 \mathrm{~A}$ & Specific conductance, lab., elec. & $\mu \mathrm{S} / \mathrm{cm}$ & 25 & RU & 1 & \\
\hline 1269 & $90095 \mathrm{~B}$ & Specific conductance, lab., LIS, elec. & $\mu \mathrm{S} / \mathrm{cm}$ & 50 & RU & .5 & \\
\hline 62 & $01080 \mathrm{~A}$ & Strontium, DIS, AA & $\mu \mathrm{g} / \mathrm{L}$ & 25 & FA & 10 & \\
\hline 652 & $01080 \mathrm{~B}$ & Strontium, DIS, ICP & $\mu g / L$ & 100 & FA & .5 & \\
\hline 290 & $01082 \mathrm{~A}$ & Strontium, WWR, AA & $\mu g / L$ & 50 & RA & 10 & 1735 \\
\hline 1572 & $00945 \mathrm{G}$ & Sulfate, DIS, IC & $\mathrm{mg} / \mathrm{L}$ & 50 & FU & .1 & \\
\hline 1263 & $00945 \mathrm{E}$ & Sulfate, LIS, DIS, IC & $\mathrm{mg} / \mathrm{L}$ & 50 & FU & .01 & \\
\hline 89 & 00745 A & Sulfide, WWR iodometric & $\mathrm{mg} / \mathrm{L}$ & 250 & LC0089 & .05 & \\
\hline 492 & 01057 A & Thallium, DIS, GFAA & $\mu \mathrm{g} / \mathrm{L}$ & 50 & FAB & .5 & \\
\hline 50 & $00076 \mathrm{~A}$ & Turbidity as NTU, nephelometric & Units & 100 & LC0050 & .1 & \\
\hline 1210 & $01085 \mathrm{D}$ & $\begin{array}{l}\text { Vanadium, DIS, catalytic } \\
\text { oxidation, ASF }\end{array}$ & $\mu \mathrm{g} / \mathrm{L}$ & 50 & FU & 1 & \\
\hline 653 & $01085 \mathrm{~B}$ & Vanadium, DIS, ICP & $\mu g / L$ & 100 & $\mathrm{FA}$ & 6 & \\
\hline 67 & $01090 \mathrm{~A}$ & Zinc, DIS, AA & $\mu g / L$ & 25 & FA & 10 & \\
\hline 1257 & $01090 \mathrm{D}$ & Zinc, DIS, GFAA & $\mu \mathrm{g} / \mathrm{L}$ & 250 & FAB & .5 & \\
\hline 671 & $01090 \mathrm{~B}$ & Zinc, DIS, ICP & $\mu g / L$ & 100 & FA & 3 & \\
\hline 296 & $01092 \mathrm{~A}$ & Zinc, WWR, AA & $\mu g / L$ & 50 & RA & 10 & 1735 \\
\hline
\end{tabular}

${ }^{1}$ See table 4.

${ }^{2} \mathrm{LC} 1735$, in-bottle digestion, is automatically called in for those samples requiring digestion prior to analysis for whole-water recoverable parameters.

LC 124, USEPA digestion, is automatically called in as a sample preparation for lab codes 324 (Ca total USEPA), 325 (Mg total USEPA), 326 (Na total USEPA), and 327 ( $\mathrm{K}$ total USEPA). Only one digestion charge will be added, since all four labcodes use the same digested sample.

LC 1586, a specific digestion, is required prior to analysis of As or Se by GFAA for USEPA drinking water. This lab code is called in automatically by LC 1584 (As, USEPA total GFAA) and LC 1585 (Se, USEPA total GFAA). 

Table 8.--Inorganic determinations for sediment listed by laboratory schedule

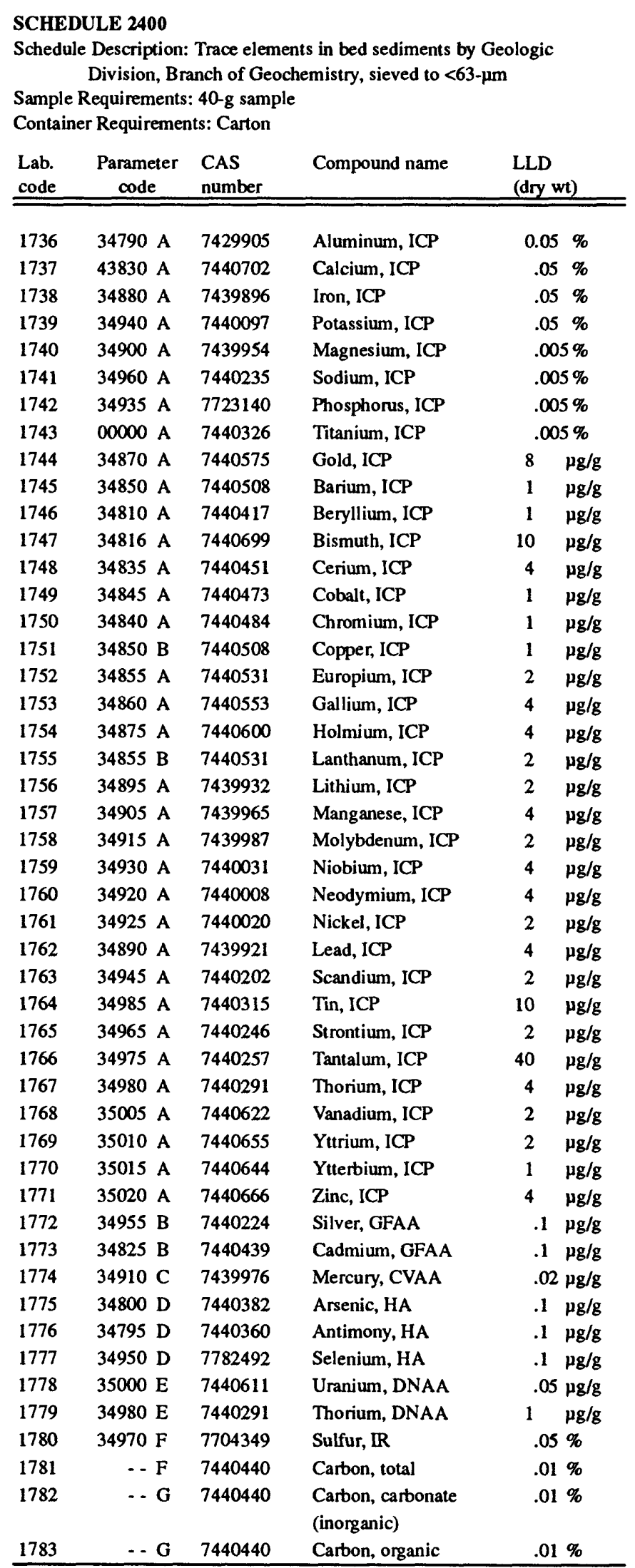





\section{SCHEDULE 146}

Schedule Description: Major cations plus iron and manganese, inductively coupled plasma, abbreviated list. Speific conductance needs to be less than $6,000 \mu \mathrm{S} / \mathrm{cm}$

Sample Requirements: 250 FA; 250 RU

Container Requirements: 250 -mL polyethylene bottle, acidrinsed; $250-\mathrm{mL}$ polyethylene bottle, field-rinsed

\begin{tabular}{|c|c|c|c|c|}
\hline $\begin{array}{l}\text { Para- } \\
\text { meter }\end{array}$ & & & $\begin{array}{l}\text { MRL } \\
\text { SPC }\end{array}$ & $\begin{array}{c}\text { MRL } \\
\text { SPC }\end{array}$ \\
\hline code & Name, phase, and method & Units & $\leq 2,000$ & $\geq 2.000$ \\
\hline $915 \mathrm{D}$ & Calcium, DIS, ICP & $\mathrm{mg} / \mathrm{L}$ & 0.02 & 0.06 \\
\hline $1046 \mathrm{D}$ & Iron, DIS, ICP & $\mu \mathrm{g} / \mathrm{L}$ & 3 & 9 \\
\hline $925 \mathrm{C}$ & Magnesium, DIS, ICP & $\mathrm{mg} / \mathrm{L}$ & .01 & .03 \\
\hline $1056 \mathrm{C}$ & Manganese, DIS, ICP & $\mu \mathrm{g} / \mathrm{L}$ & 1 & 3 \\
\hline $403 \mathrm{~A}$ & pH lab. & Units & .1 & .1 \\
\hline $955 \mathrm{D}$ & Silica as $\mathrm{SiO}_{2}$, DIS, ICP & $\mathrm{mg} / \mathrm{L}$ & .01 & .03 \\
\hline $930 \mathrm{C}$ & Sodium, DIS, ICP & $\mathrm{mg} / \mathrm{L}$ & .2 & .6 \\
\hline $095 \mathrm{~A}$ & Specific conductance, & / & 1 & \\
\hline
\end{tabular}

\section{SCHEDULE 1043}

Schedule Description: Major cations plus trace metals, inductively coupled plasma. Specific conductance needs to be less than $6,000 \mu \mathrm{S} / \mathrm{cm}$

Sample Requirements: 250 FA; 250 RU

Container Requirements: 250 -mL polyethylene bottle, acidrinsed; $250-\mathrm{mL}$ polyethylene bottle, field-rinsed

\begin{tabular}{|c|c|c|c|c|}
\hline $\begin{array}{l}\text { Para- } \\
\text { meter }\end{array}$ & & & $\begin{array}{c}\text { MRL } \\
\text { SPC }\end{array}$ & $\begin{array}{c}\text { MRL } \\
\text { SPC }\end{array}$ \\
\hline ede & Name, phase, and methed & Units & $\leq 2.000$ & $\geq 2.000$ \\
\hline 005 & C Barium, DIS, ICP & $\mu \mathrm{g} / \mathrm{L}$ & 1 & 3 \\
\hline 1010 & B Beryllium, DIS, ICP & $\mu g / L$ & .5 & 1.5 \\
\hline 1025 & D Cadmium, DIS, ICP & $\mu \mathrm{g} / \mathrm{L}$ & 1 & 3 \\
\hline 915 & D Calcium, DIS, ICP & $\mathrm{mg} / \mathrm{L}$ & .02 & .06 \\
\hline 1030 & E Chromium, DIS, ICP & $\mu \mathrm{g} / \mathrm{L}$ & 5 & 15 \\
\hline 1035 & C Colbalt, DIS, ICP & $\mu \mathrm{g} / \mathrm{L}$ & 3 & 9 \\
\hline 1040 & C Copper, DIS, ICP & $\mu \mathrm{g} / \mathrm{L}$ & 10 & 30 \\
\hline 1046 & D Iron, DIS, ICP & $\mu \mathrm{g} / \mathrm{L}$ & 3 & 9 \\
\hline 1049 & C Lead, DIS, ICP & $\mu g / L$ & 10 & 30 \\
\hline 1130 & B Lithium, DIS, ICP & $\mu g / L$ & 4 & 12 \\
\hline 925 & C Magnesium, DIS, ICP & $\mathrm{mg} / \mathrm{L}$ & .01 & .03 \\
\hline 1056 & C Manganese, DIS, ICP & $\mu g / L$ & 1 & 3 \\
\hline 1060 & A Molybdenum, DIS, ICP & $\mu g / L$ & 10 & 30 \\
\hline 1065 & E Nickel, DIS, ICP & $\mu \mathrm{g} / \mathrm{L}$ & 10 & 30 \\
\hline 403 & A pH lab., elec. & Units & .1 & .1 \\
\hline 9551 & D Silica as $\mathrm{SiO}_{2}$, DIS, ICP & $\mathrm{mg} / \mathrm{L}$ & .01 & .03 \\
\hline 1075 & C Silver, DIS, ICP & $\mu \mathrm{g} / \mathrm{L}$ & 1 & 3 \\
\hline 930 & C Sodium, DIS, ICP & $\mathrm{mg} / \mathrm{l}$ & .2 & .6 \\
\hline & A Specific conductanc & $\mu \mathrm{S} / \mathrm{cn}$ & 1 & \\
\hline 1080 & B Strontium, DIS, ICP & $\mu \mathrm{g} / \mathrm{L}$ & .5 & 1.5 \\
\hline 1085 & B Vanadium, DIS, ICP & $\mu \mathrm{g} / \mathrm{L}$ & 6 & 18 \\
\hline 1090 & B Zinc, DIS, ICP & $\mu \mathrm{g} / \mathrm{L}$ & 3 & 9 \\
\hline
\end{tabular}

\section{SCHEDULE 2701}

Schedule Description: Major inorganics in surface water for NAWQA Sample Requirements: $250 \mathrm{~mL}$, filtered acidified (FA) with $\mathrm{HNO}_{3}$ to $\mathrm{pH}<2$ $250 \mathrm{~mL}$, unfiltered nonacidified $(R U)$ $500 \mathrm{~mL}$, filtered nonacidified (FU)

Container Requirements: $250 \mathrm{~mL}$ polyethylene bottle, acid-rinsed $500-\mathrm{mL}$ polyethelene bottle, field-rinsed

\begin{tabular}{|c|c|c|c|c|}
\hline $\begin{array}{l}\text { Lab } \\
\text { code }\end{array}$ & $\begin{array}{c}\text { Para- } \\
\text { meter } \\
\text { code }\end{array}$ & $\begin{array}{r}\text { CAS } \\
\text { number }\end{array}$ & Compound name & MRL \\
\hline 15 & $00940 \mathrm{E}$ & & Chloride, DIS & $0.1 \mathrm{mg} / \mathrm{L}$ \\
\hline 27 & $70300 \mathrm{~A}$ & & ROE DIS @ $180^{\circ} \mathrm{C}$ & $1.0 \mathrm{mg} / \mathrm{L}$ \\
\hline 54 & $00935 \mathrm{~A}$ & & Potassium, DIS & $.1 \mathrm{mg} / \mathrm{L}$ \\
\hline 68 & $00403 \mathrm{~A}$ & & pH, Laboratory & .1 \\
\hline 69 & $90095 \mathrm{~A}$ & & Specific Conductance, lab. & $1.0 \mu \mathrm{S} / \mathrm{cm}$ \\
\hline 70 & $90410 \mathrm{~A}$ & & Alkal inity, as $\mathrm{CaCO}_{3}$, lab. & $1.0 \mathrm{mg} / \mathrm{L}$ \\
\hline 645 & $01046 \mathrm{D}$ & & Iron, DIS & $3.0 \mu \mathrm{g} / \mathrm{L}$ \\
\hline 648 & $01056 \mathrm{C}$ & & Manganese, DIS & $1.0 \mu \mathrm{g} / \mathrm{L}$ \\
\hline 659 & $00915 \mathrm{D}$ & & Calcium, DIS & $.02 \mathrm{mg} / \mathrm{L}$ \\
\hline 663 & $00925 \mathrm{C}$ & & Magnesium, DIS & $.01 \mathrm{mg} / \mathrm{L}$ \\
\hline 667 & $00955 \mathrm{D}$ & & Silica, DIS & $.01 \mathrm{mg} / \mathrm{L}$ \\
\hline 675 & $00930 \mathrm{C}$ & & Sodium, DIS & $.2 \mathrm{mg} / \mathrm{L}$ \\
\hline 1572 & $00945 \mathrm{G}$ & & Sulfate, DIS & $.1 \mathrm{mg} / \mathrm{L}$ \\
\hline 1573 & $00950 \mathrm{E}$ & & Fluoride, DIS & $.1 \mathrm{mg} / \mathrm{L}$ \\
\hline
\end{tabular}

\section{SCHEDULE 2702}

Schedule Description: Nutrients in surface water for NAWQA Sample Requirements: $125 \mathrm{~mL}$ water passed through $0.45-\mu \mathrm{m}$ filter, chilled (FC) @ 4 deg. $\mathrm{C}$ (packed in ice), w/ $/ \mathrm{HgCl}_{2}$ ampule added, $125 \mathrm{~mL}$ unfiltered chilled (RC)@ 4 deg. C (packed in ice) $\mathrm{w} / \mathrm{HgCl}_{2}$ ampule added

Container Requirements: $125-\mathrm{mL}$ brown polyethylene bottle, field-rinsed

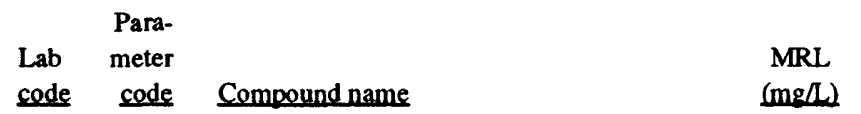

16000613 B Nitrogen, nitrite, as N, DIS 0.01

16200671 B Phosphorus, orthophosphate, as P, DIS $\quad .01$

22800631 B Nitrogen, nitrate+nitrite, as N, DIS $\quad .05$

30100608 B Nitrogen, ammonia, as N, DIS

$168500666 \mathrm{C}$ Phosphorus, as P, DIS .01

$168700623 \mathrm{C}$ Nitrogen, ammoniatorganic, as N, DIS $\quad .20$

$168800625 \mathrm{C}$ Nitrogen, ammonia+organic, as N, total $\quad .20$

$168600665 \mathrm{C}$ Phosphorus, as $\mathrm{P}$, total .01 


\section{SCHEDULE 2703}

Schedule Description: Trace elements in ground water filtered through a 0.45- $\mathrm{um}$ filter for NAWQA (Protocol Memo 7-9-93, Ver 1.3)

Sample Requirements: $250 \mathrm{~mL}$ unfiltered ground water, nonacidified (RU) $250 \mathrm{~mL}$, filtered $(0.45-\mu \mathrm{m})$, acidified with nitric acid to $\mathrm{pH}$ of $<2$ (FA)

Container Requirements: $250-\mathrm{mL}$ polyethylene bottle, field-rinsed, 250-mL polyethylene bottle, acid-rinsed

\begin{tabular}{|c|c|c|c|c|c|}
\hline $\begin{array}{l}\text { Lab- } \\
\text { code }\end{array}$ & $\begin{array}{l}\text { Para- } \\
\text { meter } \\
\text { code }\end{array}$ & $\begin{array}{r}\text { CAS } \\
\text { number }\end{array}$ & Compound name & & MRL \\
\hline 0068 & $00403 \mathrm{~A}$ & & pH, laboratory & 0.1 & \\
\hline 0069 & $90095 \mathrm{~A}$ & & Specific conductance, lab. & 1 & $\mu \mathrm{S} / \mathrm{cm}$ \\
\hline 0112 & $01000 \mathrm{~B}$ & 7440382 & Arsenic, DIS, & 1 & $\mu \mathrm{g} / \mathrm{L}$ \\
\hline 1784 & $01106 \mathrm{G}$ & 7429905 & Aluminum, DIS, ICP/MS & 1 & $\mu \mathrm{g} / \mathrm{L}$ \\
\hline 1785 & $01095 \mathrm{G}$ & 7440360 & Antimony, DIS, ICPMS & 1 & $\mu g / L$ \\
\hline 1786 & $01005 \mathrm{G}$ & 7440393 & Barium, DIS, ICPMS & 1 & $\mu g / L$ \\
\hline 1787 & $01010 \mathrm{G}$ & 7440417 & Beryllium, DIS, ICP/MS & 1 & $\mu g / L$ \\
\hline 1788 & $01025 \mathrm{G}$ & 7440439 & Cadmium, DIS, ICP/MS & 1 & $\mu g / L$ \\
\hline 1789 & $01030 \mathrm{G}$ & 7440473 & Chromium, DIS, ICP/MS & 1 & $\mu g / L$ \\
\hline 1790 & $01035 \mathrm{G}$ & 7440484 & Cobalt, DIS, ICP/MS & 1 & $\mu g / L$ \\
\hline 1791 & $01040 \mathrm{G}$ & 7440508 & Copper, DIS, ICPMS & 1 & $\mu g / L$ \\
\hline 1792 & $01049 \mathrm{G}$ & 7439921 & Lead, DIS, ICPMS & 1 & $\mu g / L$ \\
\hline 1793 & $01056 \mathrm{G}$ & 7439965 & Manganese, DIS, ICP/MS & 1 & $\mu \mathrm{g} / \mathrm{L}$ \\
\hline 1794 & $01060 \mathrm{G}$ & 7439987 & Molybdenum, DIS, ICP/MS & 1 & $\mu \mathrm{g} / \mathrm{L}$ \\
\hline 1795 & $01065 \mathrm{G}$ & 7440020 & Nickel, DIS, ICP/MS & 1 & $\mu g / L$ \\
\hline 0087 & $01145 \mathrm{~A}$ & 7782492 & Selenium, DIS & 1 & $\mu g / L$ \\
\hline 1796 & $01075 \mathrm{G}$ & 7440224 & Silver, DIS, ICP/MS & 1 & $\mu \mathrm{g} / \mathrm{L}$ \\
\hline 1797 & $22703 \mathrm{G}$ & 7440611 & Uranium, DIS, ICP/MS & 1 & $\mu \mathrm{g} / \mathrm{L}$ \\
\hline 1798 & $01090 \mathrm{G}$ & 7440666 & Zinc, DIS, ICP/MS & 1 & $\mu g / L$ \\
\hline
\end{tabular}

\section{SCHEDULE 2750}

Schedule Description: Major inorganics in ground water for NAWQA

Sample Requirements: $250 \mathrm{~mL}$, filtered acidified (FA) $w / \mathrm{HNO}_{3}$ to $\mathrm{pH}<2$ $250 \mathrm{~mL}$, unfiltered nonacidified (RU)

$500 \mathrm{~mL}$, filtered nonacidified (FU)

Container Requirements: 250 and $500-\mathrm{mL}$ polyethylene bottles, field-rinsed $250-\mathrm{mL}$ polyethylene bottle, acid-rinsed

$\begin{array}{rc}\begin{array}{c}\text { Lab- } \\ \text { code }\end{array} & \begin{array}{c}\text { Para- } \\ \text { meter } \\ \text { code }\end{array} \\ 15 & 00940 \mathrm{E} \\ 27 & 70300 \mathrm{~A} \\ 54 & 00935 \mathrm{~A} \\ 68 & 00403 \mathrm{~A} \\ 69 & 90095 \mathrm{~A} \\ 70 & 90410 \mathrm{~A} \\ 645 & 01046 \mathrm{D} \\ 648 & 01056 \mathrm{C} \\ 659 & 00915 \mathrm{D} \\ 663 & 00925 \mathrm{C} \\ 667 & 00955 \mathrm{D} \\ 675 & 00930 \mathrm{C} \\ 1246 & 71870 \mathrm{E} \\ 1572 & 00945 \mathrm{G} \\ 1573 & 00950 \mathrm{E}\end{array}$

\section{SCHEDULE 2752}

Schedule Description: Nutrients in ground water for NAWQA Sample Requirements: $125 \mathrm{~mL}$ water passed through $0.45-\mu \mathrm{m}$ filter, chilled, (FC) @ 4 deg. $\mathrm{C}$ (packed in ice), w/ $/ \mathrm{HgCl}_{2}$ ampule added Container Requirements: $125-\mathrm{mL}$ brown polyethylene bottle, field-rinsed

$\begin{array}{rllr}\begin{array}{c}\text { Lab- } \\ \text { code }\end{array} & \begin{array}{c}\text { Para- } \\ \text { meter } \\ \text { code }\end{array} & \begin{array}{r}\text { MRL } \\ \text { (ma } \Omega \text { Compound name }\end{array} \\ 160 & 00613 \text { B Nitrogen, nitrite, as N, DIS } & 0.01 \\ 162 & 00671 \text { B Phosphons, orthophosphate, as P, DIS } & .01 \\ 228 & 00631 \text { B Nitrogen, nitrate+nitrite, as N, DIS } & .05 \\ 301 & 00608 \text { B Nitrogen, ammonia, as N, DIS } & .01 \\ 1685 & 00666 \text { C Phosphonus, as P, DIS } & .01 \\ 1687 & 00623 \text { C Nitrogen, ammonia+organic, as N, DIS } & .20\end{array}$


Table 10.--Inorganic determinations for tissue listed by laboratory schedule

\section{SCHEDULE 2200}

Schedule Description: Trace elements in tissue samples (fish, liver, or corbicula)

Sample Requirements: $20 \mathrm{~g}$ of sample, frozen, shipped @ $4 \mathrm{deg} . \mathrm{C}$

(packed in ice)

Container Requirements: Ziploc bag

\begin{tabular}{|c|c|c|c|c|c|}
\hline \multirow{2}{*}{$\begin{array}{l}\begin{array}{c}\text { Lab. } \\
\text { code }\end{array} \\
6000\end{array}$} & \multicolumn{2}{|c|}{$\begin{array}{l}\text { Parameter } \\
\text { code }\end{array}$} & $\begin{array}{c}\text { CAS } \\
\text { number }\end{array}$ & Compound name & $\begin{array}{r}\text { MRL } \\
(\mu g / g) \\
\text { dry }\end{array}$ \\
\hline & -- & A & - & Aluminum, ICP & 1.0 \\
\hline 6001 & $\cdots$ & A & - & Barium, ICP & .1 \\
\hline 6003 & $\cdots$ & A & - & Boron, ICP & .2 \\
\hline 6005 & $\cdots$ & A & - & Chromium, ICP & .5 \\
\hline 6007 & $\cdots$ & A & - & Copper, ICP & .5 \\
\hline 6008 & - & $\mathbf{A}$ & - & Iron, ICP & 1 \\
\hline 6010 & - & $\mathbf{A}$ & - & Manganese, ICP & .1 \\
\hline 6014 & -- & A & - & Strontium, ICP & .1 \\
\hline 6016 & $\cdots$ & $\mathbf{A}$ & - & Zinc, ICP & .5 \\
\hline 6018 & $\cdots$ & B & - - & Antimony, ICP/MS & .1 \\
\hline 6019 & $\ldots$ & B & - & Arsenic, ICP/MS & .1 \\
\hline 6021 & $\cdots$ & B & - & Beryllium, ICP/MS & .1 \\
\hline 6023 & - & B & - & Cadmium, ICP/MS & .1 \\
\hline 6025 & - & B & - & Cobalt, ICP/MS & .1 \\
\hline 6028 & - & B & - & Lead, ICP/MS & .1 \\
\hline 6030 & $\cdots$ & B & - & Molybdenum, ICP/MS & .1 \\
\hline 6031 & - & B & - - & Nickel, ICP/MS & .1 \\
\hline 6032 & -- & B & $\cdots$ & Selenium, ICP/MS & .1 \\
\hline 6033 & -- & B & $\cdots$ & Silver, ICPMS & .1 \\
\hline 6036 & - & B & -- & Uranium, ICP/MS & .1 \\
\hline 6037 & - & B & - & Vanadium, ICP/MS & .1 \\
\hline 6046 & $\cdots$ & $\mathbf{A}$ & - & Mercury, CVAA & .1 \\
\hline 6047 & $\cdots$ & $\mathbf{A}$ & $\cdots$ & Water, percent & 0 \\
\hline
\end{tabular}


Table 11.--Gross organic determinations listed alphabetically

\begin{tabular}{|c|c|c|c|c|c|c|}
\hline $\begin{array}{l}\text { Lab. } \\
\text { code }\end{array}$ & $\begin{array}{l}\text { Parameter } \\
\text { code }\end{array}$ & Name and phase & Units & $\begin{array}{l}\text { Volume } \\
\text { needed }\end{array}$ & $\begin{array}{c}\text { Sample } \\
\text { designa- } \\
\text { tion }\end{array}$ & MRL \\
\hline \multicolumn{7}{|c|}{ Sediment } \\
\hline 503 & $686 \mathrm{C}$ & Carbon, inorganic, BTM, modified Van Slyke & $\mathrm{g} / \mathrm{kg}$ & $10 \mathrm{~g}$ & $\mathrm{CC}$ & 0.1 \\
\hline 133 & $693 \mathrm{~A}$ & $\begin{array}{l}\text { Carbon, total, inorganic + organic, BTM, } \\
\text { induction furnace }\end{array}$ & $\mathrm{g} / \mathrm{kg}$ & $10 \mathrm{~g}$ & $\mathrm{CC}$ & .1 \\
\hline 531 & $557 \mathrm{~A}$ & Oil \& grease, BTM, extraction gravimetric & $\mathrm{mg} / \mathrm{kg}$ & $10 \mathrm{~g}$ & $\mathrm{CC}$ & 1,000 \\
\hline \multicolumn{7}{|l|}{ Water } \\
\hline 306 & $691 \mathrm{~A}$ & Carbon, inorganic, DIS & $\mathrm{mg} / \mathrm{L}$ & $100 \mathrm{~mL}$ & DIC & .1 \\
\hline 19 & $685 \mathrm{~A}$ & Carbon, inorganic, total & $\mathrm{mg} / \mathrm{L}$ & $100 \mathrm{~mL}$ & TIC & .1 \\
\hline 113 & $681 \mathrm{~A}$ & Carbon, organic, DIS & $\mathrm{mg} / \mathrm{L}$ & $100 \mathrm{~mL}$ & DOC & .1 \\
\hline 305 & $689 \mathrm{~A}$ & Carbon, organic, SUS, wet oxidation & $\mathrm{mg} / \mathrm{L}$ & 1 Filter & SOC & .1 \\
\hline 114 & $680 \mathrm{~A}$ & Carbon, organic, total, wet oxidation & $\mathrm{mg} / \mathrm{L}$ & $100 \mathrm{~mL}$ & TOC & .1 \\
\hline 96 & $38260 \mathrm{~A}$ & MBAS, WWR, colorimetric & $\mathrm{mg} / \mathrm{L}$ & $250 \mathrm{~mL}$ & $\mathrm{RCB}$ & .01 \\
\hline 127 & $556 \mathrm{~A}$ & Oil \& grease, total, extraction gravimetric & $\mathrm{mg} / \mathrm{L}$ & $1 \mathrm{~L}$ & $\mathrm{LC} 0127$ & 1 \\
\hline 52 & $32730 \mathrm{~A}$ & Phenols, total, colorimetric & $\mu \mathrm{g} / \mathrm{L}$ & $1 \mathrm{~L}$ & $\mathrm{LC} 0052$ & 1 \\
\hline 138 & 32240 A & Tannin \& lignin, recoverable, colorimetric & $\mathrm{mg} / \mathrm{L}$ & $100 \mathrm{~mL}$ & RCB & .1 \\
\hline
\end{tabular}

${ }^{1}$ See table 4. 



\section{SCHEDULE 80}

Schedule Description: Organochlorine herbicides, recoverable from bed material analyzed by GC/ECD

Sample Requirements: 200 g, BGC, chilled \& maintained @ 4 deg. C Container Requirement: 1-L, amber, 33-mL neck, pesticide glass bottle, cleaned and burned from NWQL

\begin{tabular}{lccrl}
$\begin{array}{l}\text { Lab. } \\
\text { code }\end{array}$ & $\begin{array}{c}\text { Parameter } \\
\text { code }\end{array}$ & \multicolumn{1}{c}{$\begin{array}{l}\text { CAS } \\
\text { number }\end{array}$} & Compoun \\
& & & \\
0375 & $39731 \mathrm{~A}$ & 94757 & $2,4-\mathrm{D}$ \\
0376 & $39741 \mathrm{~A}$ & 93765 & $2,4,5-\mathrm{T}$ \\
0377 & $39761 \mathrm{~A}$ & 93721 & Silvex \\
0403 & $34609 \mathrm{~A}$ & 105679 & $2,4-\mathrm{DP}$ \\
0750 & $38930 \mathrm{~A}$ & 918021 & Picloram \\
0751 & $38931 \mathrm{~A}$ & 918009 & Dicamb
\end{tabular}

\section{SCHEDULE 1305}

Schedule Description: Chlorophenoxy acid herbicides, recoverable from bottom material, analyzed by GC/ECD

Sample Requirements: 200 g, BGC, chilled \& maintained @ 4 deg. C Container Requirement: 1-L, amber, 33-mL neck, pesticide glass bottle, cleaned and burned from NWQL

$\begin{array}{lcclr}\begin{array}{l}\text { Lab. } \\ \text { code }\end{array} & \begin{array}{c}\text { Parameter } \\ \text { code }\end{array} & \begin{array}{l}\text { CAS } \\ \text { number }\end{array} & \text { Compound name } & \begin{array}{r}\text { MRL } \\ (\mathrm{\mu g} / \mathrm{kg})\end{array} \\ 0375 & 39731 \text { A } & 94757 & 2,4-\mathrm{D} & \\ 0376 & 39741 \text { A } & 93765 & 2,4,5-\mathrm{T} & 0.1 \\ 0377 & 39761 \mathrm{~A} & 93721 \text { Silvex } & .1 \\ 0403 & 34609 \text { A } & 105679 & 2,4-\mathrm{DP} & .1 \\ & & & & .1\end{array}$

\section{SCHEDULE 1320}

Schedule Description: Organophosphate pesticides, recoverable from bottom material, analyzed by GC/FPD

Sample Requirements: 200 g, BGC, chilled \& maintained @ 4 deg. C Container Requirement: 1-L, amber, 33-mL neck, pesticide glass bottle, cleaned and burned from NWQL

$\begin{array}{lcrll}\begin{array}{l}\text { Lab. } \\ \text { code }\end{array} & \begin{array}{c}\text { Parameter } \\ \text { code }\end{array} & \begin{array}{c}\text { CAS } \\ \text { number }\end{array} & \text { Compound name } \\ & & & & \\ 0385 & 39571 \text { A } & 333415 & \text { Diazinon } \\ 0386 & 39399 \text { A } & 563122 & \text { Ethion } \\ 0387 & 39531 \text { A } & 121755 & \text { Malathion } \\ 0388 & 39601 \text { A } & 298000 & \text { Methylparathion } \\ 0390 & 39541 \text { A } & 56382 & \text { Parathion } \\ 0391 & 39787 \text { A } & 786196 & \text { Trithion }\end{array}$

\section{MRL} $(\mathrm{\mu g} / \mathrm{kg})$

0.1
.1
.1
.1
.1
.1

\section{SCHEDULE 1325}

Schedule Description: Organochlorine pesticides with PCB's and PCN's, recoverable from bottom material, analyzed by GC/ECD Sample Requirements: $200 \mathrm{~g}$, BGC, chilled \& maintained at $4 \mathrm{deg}$. C Container Requirement: 1-L, amber, 33-mL neck, pesticide glass bottle, cleaned and burned from NWQL

\begin{tabular}{lcrrl}
$\begin{array}{l}\text { Lab. } \\
\text { code }\end{array}$ & $\begin{array}{c}\text { Parameter } \\
\text { code }\end{array}$ & \multicolumn{1}{c}{ CAS } \\
number & Compound name \\
0342 & 81886 A & 72560 & Perthane \\
0346 & 39389 A & 15297 & Endosulfan \\
0361 & 39333 A & 309002 & Aldrin \\
0362 & 39351 A & 57749 & Chlordane \\
0363 & 39363 A & 72548 & DDD \\
0364 & 39368 A & 72559 & DDE \\
0365 & 39373 A & 50293 & DDT \\
0366 & 39383 A & 60571 & Dieldrin \\
0367 & 39393 A & 72208 & Endrin \\
0368 & 39413 A & 76448 & Heptachlor \\
0369 & 39423 A & 1024573 & Haptachlor epoxide \\
0370 & 39343 A & 58899 & Lindane \\
0371 & 39403 A & 8001352 & Toxaphene \\
0394 & 39519 A & 12767792 & PCB's, gross \\
0395 & 39251 A & 25104556 & PCN's, gross \\
0401 & 39481 A & 72435 & Methoxychlor \\
0545 & 39758 A & 2385855 & Mirex
\end{tabular}

MRL (1)g/kg)

\section{SCHEDULE 1335}

Schedule Description: Organochlorine and organophosphate pestiides, with PLB's and PLN's recoverable from bottom material

Sample Requirements: $200 \mathrm{~g}$, BGC, chilled \& maintained @ 4 deg. C

Container Requirement: 1-L, amber, 33-mL neck, pesticide glass bottle, cleaned and burned from NWQL

\begin{tabular}{|c|c|c|c|}
\hline $\begin{array}{l}\text { Lab. } \\
\text { code }\end{array}$ & $\begin{array}{l}\text { Parameter } \\
\text { code }\end{array}$ & $\begin{array}{l}\text { CAS } \\
\text { number }\end{array}$ & Compound name \\
\hline 0342 & $81886 \mathrm{~A}$ & 72560 & Perthane \\
\hline 0346 & 39389 A & 115297 & Endosulfan \\
\hline 0361 & $39333 \mathrm{~A}$ & 309002 & Aldrin \\
\hline 0362 & $39351 \mathrm{~A}$ & 57749 & Chlordane \\
\hline 0363 & $39363 \mathrm{~A}$ & 72548 & DDD \\
\hline 0364 & $39368 \mathrm{~A}$ & 72559 & DDE \\
\hline 0365 & $39373 \mathrm{~A}$ & 50293 & DDT \\
\hline 0366 & $39383 \mathrm{~A}$ & 60571 & Dieldrin \\
\hline 0367 & $39393 \mathrm{~A}$ & 72208 & Endrin \\
\hline 0368 & 39413 A & 76448 & Heptachlor \\
\hline 0369 & 39423 A & 1024573 & Heptachlor epoxide \\
\hline 0370 & 39343 A & 58899 & Lindane \\
\hline 0371 & $39403 \mathrm{~A}$ & 8001352 & Toxaphene \\
\hline 0385 & 39571 A & 333415 & Diazinon \\
\hline 0386 & 39399 A & 563122 & Ethion \\
\hline 0387 & $39531 \mathrm{~A}$ & 121755 & Malathion \\
\hline 0388 & 39601 A & 298000 & Methylparathion \\
\hline 0390 & $39541 \mathrm{~A}$ & 56382 & Parathion \\
\hline 0391 & $39787 \mathrm{~A}$ & 786196 & Trithion \\
\hline 0394 & 39519 A & 12767792 & PCB's, gross \\
\hline 0395 & $39251 \mathrm{~A}$ & 25104556 & PCN's, gross \\
\hline 0401 & $39481 \mathrm{~A}$ & 72435 & Methoxychlor \\
\hline 0545 & $39758 \mathrm{~A}$ & 2385855 & Mirex \\
\hline
\end{tabular}

MRL (119/Ka) 


\section{SCHEDULE 1382}

Schedule Description: Organic compound profile, recoverable from bottom material, as determined from a methylene chloride extraction and analyzed by GC/FID

Sample Requirements: $200 \mathrm{~g}$, BGC, chilled \& maintained @ 4 deg. C Container Requirement: 1-L, amber, 33-mL neck, pesticide glass bottle, cleaned and burned from NWQL

$\begin{array}{lcccc}\begin{array}{l}\text { Lab. } \\ \text { code }\end{array} & \begin{array}{c}\text { Parameter } \\ \text { code }\end{array} & \begin{array}{l}\text { CAS } \\ \text { number }\end{array} & \begin{array}{c}\text { MRL } \\ \text { (ug/kg) }\end{array} \\ 1236 & 99475 \text { A } & \text { BTM GC/FID profile } & \text { N/A }\end{array}$

\section{SCHEDULE 1384}

Schedule Description: Organic compounds, acid and base/neutral, recoverable from bottom material using methylene chloride and GCMS technology

Sample Requirements: 200 g。 BGC, chilled \& maintained @ 4 deg. C

Container Requirement: 1-L, amber, 33-mL neck, pesticide glass bottle, cleaned and burned from NWQL

\begin{tabular}{|c|c|c|c|c|}
\hline $\begin{array}{l}\text { Lab. } \\
\text { code }\end{array}$ & $\begin{array}{c}\text { Parameter } \\
\text { code }\end{array}$ & $\begin{array}{l}\text { CAS } \\
\text { number }\end{array}$ & Compound name & $\begin{array}{c}\text { MRL } \\
(\mathbf{L} \mathrm{g} / \mathrm{kg})\end{array}$ \\
\hline 1044 & $34455 \mathrm{~A}$ & 59507 & 3-Chloro-3-methylphenol & 600 \\
\hline 1045 & 34589 A & 95578 & 2-Chlorophenol & 200 \\
\hline 1046 & 34604 A & 120832 & 2,4-Dichlorophenol & 200 \\
\hline 1047 & 34609 B & 105679 & 2,4-Dimethylphenol & 200 \\
\hline 1048 & $34660 \mathrm{~A}$ & 534521 & 2-Methyl-4,6-dinitrophenol & 600 \\
\hline 1049 & 34619 A & 51285 & 2,4-Dinitrophenol & 600 \\
\hline 1050 & 34594 A & 88755 & 2-Nitrophenol & 200 \\
\hline 1051 & $34649 \mathrm{~A}$ & 100027 & 4-Nitrophenol & 600 \\
\hline 1052 & $39061 \mathrm{~A}$ & 87865 & Pentachlorophenol & 600 \\
\hline 1053 & 34695 A & 108952 & Phenol & 200 \\
\hline 1054 & 34624 A & 88062 & 2,4,6-Trichlorophenol & 600 \\
\hline 1112 & 34208 A & 83329 & Acenaphthene & 200 \\
\hline 1113 & $34203 \mathrm{~A}$ & 208968 & Acenaphthylene & 200 \\
\hline 1114 & $34223 \mathrm{~A}$ & 120127 & Anthracene & 200 \\
\hline 1116 & 34529 A & 56553 & Benzo[a]anthracene & 400 \\
\hline 1117 & $34233 \mathrm{~A}$ & 205992 & Benzo[b]fluoranthene & 400 \\
\hline 1118 & $34245 \mathrm{~A}$ & 207089 & Benzo[k]fluoranthene & 400 \\
\hline 1119 & $34250 \mathrm{~A}$ & 50328 & Benzo[a]pyrene & 400 \\
\hline 1120 & 34524 A & 191242 & Benzo[g,h,i]perylene & 400 \\
\hline 1121 & 34295 A & 85687 & Butyl benzyl phthalate & 200 \\
\hline 1122 & $34281 \mathrm{~A}$ & 111911 & Bis(2-chloroethoxy)methane & 200 \\
\hline 1123 & $34276 \mathrm{~A}$ & 111444 & Bis(2-chloroethyl)ether & 200 \\
\hline 1124 & 34286 A & 708601 & Bis(2-chloroisopropyl)ether & 200 \\
\hline 1125 & 34639 A & 101553 & 4-Bromophenylphenylether & 200 \\
\hline 1126 & 34584 A & 91587 & 2-Chloronaphthalene & 200 \\
\hline 1127 & 34644 A & 7005723 & 4-Chlorophenylphenylether & 200 \\
\hline 1128 & $34323 \mathrm{~A}$ & 218019 & Chrysene & 400 \\
\hline 1129 & 34559 A & 53703 & 1,2,5,6-Dibenz $[\mathrm{a}, \mathrm{h}]$ anthracene & 400 \\
\hline 1130 & $39112 \mathrm{~A}$ & 84742 & Di-n-butyl phthalate & 200 \\
\hline 1140 & 34539 A & 95501 & 1,2-Dichlorobenzene & 200 \\
\hline 1141 & 34569 A & 541731 & 1,3-Dichlorobenzene & 200 \\
\hline 1142 & 34574 A & 106467 & 1,4-Dichlorobenzene & 200 \\
\hline 1144 & 34339 A & 84662 & Diethyl phthalate & 200 \\
\hline 1145 & 34344 A & 131113 & Dimethyl phthalate & 200 \\
\hline 1146 & 34614 A & 121142 & 2,4-Dinitrotoluene & 200 \\
\hline 1147 & 34629 A & 606202 & 2,6-Dinitrotoluene & 200 \\
\hline 1148 & 34599 A & 117840 & Di-n-octyl phthalate & 400 \\
\hline 1149 & $39102 \mathrm{~A}$ & 117817 & Bis(2-ethylhexyl)pht & 200 \\
\hline 1150 & 34384 A & 86737 & Fluorene & 200 \\
\hline
\end{tabular}

MRL
SCHEDULE 1384--Continued

\begin{tabular}{|c|c|c|c|c|}
\hline $\begin{array}{l}\text { Lab. } \\
\text { code }\end{array}$ & $\begin{array}{c}\text { Parameter } \\
\text { code }\end{array}$ & $\begin{array}{l}\text { CAS } \\
\text { number }\end{array}$ & Compound name & $\begin{array}{l}\text { MRL } \\
\text { (lug/kg }\end{array}$ \\
\hline 1151 & 34379 A & 206440 & Fluoranthene & 200 \\
\hline 1152 & $39701 \mathrm{~A}$ & 118741 & Hexachlorobenzene & 200 \\
\hline 1153 & $39705 \mathrm{~A}$ & 87683 & Hexachlorobutadiene & 200 \\
\hline 1154 & 34389 A & 77474 & Hexachlorocyclopentadiene & 200 \\
\hline 1155 & 34399 A & 67721 & Hexachloroethane & 200 \\
\hline 1156 & 34406 A & 193395 & Indeno (1,2,3-cd) pyrene & 400 \\
\hline 1157 & 34411 A & 78591 & Isophorone & 200 \\
\hline 1158 & 34445 A & 91203 & Naphthalene & 200 \\
\hline 1159 & 34450 A & 98953 & Nitrobenzene & 200 \\
\hline 1160 & 34441 A & 62759 & N-Nitrosodimethylamine & 200 \\
\hline 1161 & 34436 A & 86306 & N-Nitrosodiphenylamine & 200 \\
\hline 1162 & 34431 A & 621647 & N-Nitrodi-n-propylamine & 200 \\
\hline 1163 & 34464 A & 85018 & Phenanthrene & 200 \\
\hline 1164 & 34472 A & 129000 & Pyrene & 200 \\
\hline 1166 & 34554 A & 120821 & 1,2,4-Trichlorobenzene & 200 \\
\hline
\end{tabular}

MRL 200 200 200 200 200 200 200 200 200 


\section{SCHEDULE 1386}

Schedule Description: Organic compounds, acid and base/neutral, recoverable from bottom material using methylene chloride and GC/MS technology with unknowns tentatively identified

Sample Requirements: $200 \mathrm{~g}$, BGC, chilled \& maintained @ $4 \mathrm{deg} . \mathrm{C}$ Container Requirement: 1-L, amber, 33-mL neck, pesticide glass bottle, cleaned and burned from NWQL

\begin{tabular}{|c|c|c|c|c|}
\hline ab. & $\begin{array}{l}\text { Parameter } \\
\text { code }\end{array}$ & $\begin{array}{l}\text { CAS } \\
\text { number }\end{array}$ & Compound name & 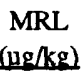 \\
\hline 044 & $34455 \mathrm{~A}$ & 59507 & 4-Chloro-3-methylphenol & 600 \\
\hline 1045 & 34589 A & 95578 & 2-Chlorophenol & 200 \\
\hline 1046 & 34604 A & 120832 & 2,4-Dichlorophenol & 200 \\
\hline 1047 & 34609 B & 105679 & 2,4-Dimethylphenol & 200 \\
\hline 1048 & $34660 \mathrm{~A}$ & 534521 & 2-Methyl-4,6-dinitrophenol & 600 \\
\hline 1049 & 34619 A & 51285 & 2,4-Dinitrophenol & 600 \\
\hline 1050 & 34594 A & 88755 & 2-Nitrophenol & 200 \\
\hline 1051 & 34649 A & 100027 & 4-Nitrophenol & 600 \\
\hline 1052 & $39061 \mathrm{~A}$ & 87865 & Pentachlorophenol & 600 \\
\hline 1053 & $34695 \mathrm{~A}$ & 108952 & Phenol & 200 \\
\hline 1054 & 34624 A & 88062 & 2,4,6-Trichlorophenol & 600 \\
\hline 1112 & $34208 \mathrm{~A}$ & 83329 & Acenaphthene & 200 \\
\hline 1113 & $34203 \mathrm{~A}$ & 208968 & Acenaphthylene & 200 \\
\hline 1114 & $34223 \mathrm{~A}$ & 120127 & Anthracene & 200 \\
\hline 1116 & $34529 \mathrm{~A}$ & 56553 & Benzo[a]anthracene & 400 \\
\hline 1117 & 34233 A & 205992 & Benzo[b]fluoranthene & 400 \\
\hline 1118 & 34245 A & 207089 & Benzo[k]fluoranthene & 400 \\
\hline 1119 & $34250 \mathrm{~A}$ & 50328 & Benzo[a]pyrene & 400 \\
\hline 1120 & $34524 \mathrm{~A}$ & 191242 & Benzo[g,h,i]perylene & 400 \\
\hline 1121 & 34295 A & 85687 & Butyl benzyl phthalate & 200 \\
\hline 1122 & $34281 \mathrm{~A}$ & 111911 & Bis(2-chloroethoxy)methane & 200 \\
\hline 1123 & $34276 \mathrm{~A}$ & 111444 & Bis(2-chloroethyl)ether & 200 \\
\hline 1124 & $34286 \mathrm{~A}$ & 108601 & Bis(2-chloroisopropyl)ether & 200 \\
\hline 1125 & 34639 A & 101553 & 4-Bromophenylphenylether & 200 \\
\hline 1126 & $34584 \mathrm{~A}$ & 91587 & 2-Chloronaphthalene & 200 \\
\hline 1127 & 34644 A & 7005723 & 4-Chlorophenylphenylether & 200 \\
\hline 1128 & $34323 \mathrm{~A}$ & 218019 & Chrysene & 400 \\
\hline 1129 & $34559 \mathrm{~A}$ & 53703 & 1,2,5,6-Dibenz[a,h]anthracene & 400 \\
\hline 1130 & $39112 \mathrm{~A}$ & 84742 & Di-n-butyl phthalate & 200 \\
\hline 1140 & 34539 A & 95501 & 1,2-Dichlorobenzene & 200 \\
\hline 1141 & $34569 \mathrm{~A}$ & 541731 & 1,3-Dichlorobenzene & 200 \\
\hline 1142 & $34574 \mathrm{~A}$ & 106467 & 1,4-Dichlorobenzene & 200 \\
\hline 1144 & 34339 A & 84662 & Diethyl phthalate & 200 \\
\hline 1145 & 34344 A & 131113 & Dimethyl phthalate & 200 \\
\hline 1146 & $34614 \mathrm{~A}$ & 121142 & 2,4-Dinitrotoluene & 200 \\
\hline 1147 & 34629 A & 606202 & 2,6-Dinitrotoluene & 200 \\
\hline 1148 & 34599 A & 117840 & Di-n-octyl-phthalte & 400 \\
\hline 1149 & $39102 \mathrm{~A}$ & 117817 & ylhexyl)pht & 200 \\
\hline 1150 & 34384 A & 86737 & Fluorene & 200 \\
\hline 1151 & 34379 A & 206440 & Fluoranthene & 200 \\
\hline 1152 & $39701 \mathrm{~A}$ & 118741 & Hexachlorobenzene & 200 \\
\hline 1153 & 39705 A & 87683 & Hexachlorobutadiene & 200 \\
\hline 1154 & 34389 A & 77474 & Hexachlorocyclopentadiene & 200 \\
\hline 1155 & 34399 A & 67721 & Hexachloroethane & 200 \\
\hline 1156 & 34406 A & 193395 & Indeno $(1,2,3-c d)$ pyrene & 400 \\
\hline 1157 & 34411 A & 78591 & Isophorone & 200 \\
\hline 1158 & 34445 A & 91203 & Naphthalene & 200 \\
\hline 1159 & 34450 A & 98953 & Nitrobenzene & 200 \\
\hline 1160 & 34441 A & 62759 & N-Nitrosodimet & 200 \\
\hline 1161 & 34436 A & 86306 & N-Nitrosodiphenylamine & 200 \\
\hline 1162 & 34431 A & 621647 & N-Nitrosodi-n-propylamine & 200 \\
\hline 1163 & 34464 A & 85018 & Phenanthrene & 200 \\
\hline 1164 & 34472 A & 129000 & Pyrene & 200 \\
\hline 1166 & 34554 A & 120821 & 1,2,4-Trichlorobenzene & 200 \\
\hline
\end{tabular}

\section{SCHEDULE 2501}

Schedule Description: Chlorinated organic compounds in bed sediments analyzed by the NWQL using gas chromatography

Sample Requirements: $500 \mathrm{~g}$ of bed sediment chilled @ $4 \mathrm{deg} . \mathrm{C}$ (packed in ice), fill container no more than two-thirds full to minimize breakage

Container Requirements: $500-\mathrm{mL}$ wide-mouth glass container

Lab. Para meter

CAS MRI

code code number Compound name (ng/kg)

$500100000 \mathrm{~B} \quad$-- $\quad$ Aldrin

500200000 B $\quad$-. $\quad$ Chlordane,cis.

$500300000 \mathrm{~B}$

$505400000 \mathrm{~B}$

$503600000 \mathrm{~B}$

$500800000 \mathrm{~B}$

$500900000 \mathrm{~A}$

$501000000 \mathrm{~B}$

$501100000 \mathrm{~B}$

501200000

$501300000 \mathrm{~B}$

$501400000 \mathrm{~B}$

$501500000 \mathrm{~B}$

$501800000 \mathrm{~B}$

$502600000 \mathrm{~B}$

$502700000 \mathrm{~B}$

$502200000 \mathrm{~B}$

$502000000 \mathrm{~B}$

$502100000 \mathrm{~B}$

$500600000 \mathrm{~B}$

$503700000 \mathrm{~B}$

$504200000 \mathrm{~B}$

$504400000 \mathrm{~B}$

$502300000 \mathrm{~B}$

$504100000 \mathrm{~B}$

$503900000 \mathrm{~B}$

$5038 \quad 00000 \mathrm{~B}$

$502400000 \mathrm{~B}$

$503300000 \mathrm{~B}$

$505500000 \mathrm{~B}$

$505600000 \mathrm{~B}$

$502500000 \mathrm{~B}$

$503200000 \mathrm{~B}$

$503400000 \mathrm{~B}$

$5048 \quad 00000 \mathrm{~B}$

$505300000 \mathrm{~B}$

$505700000 \mathrm{~B}$

$5058 \quad 00000 \mathrm{~B}$
-. Chlordane,trans-

-- Chloroneb

-. DCPA(Dacthal)

-- DDD,o,p'-

-- DDD,p,p'-

-- DDE,o,p'-

- $\quad$ DDE,p,p'-

- DDT,o,p'-

-- DDT,p,p'-

-. Dieldrin

-. Endosulfan I

-- Endrin

-- $\mathrm{HCH}$, alpha-

-. $\mathrm{HCH}$, beta-

-- $\quad \mathrm{HCH}$, gamma-(Lindane)

-. Heptachlor

-. Heptachlor epoxide

-- Hexachlorobenzene

-. Isodrin

- Methoxychlor,o,p'-

-. Methoxychlor,p,p'-

-. $\quad$ Mirex

-. Nonachlor, cis-

-. Nonachlor, trans-

-. Oxychlordane

-. PCB's, total

-. Pentachloroanisole

- Permethrin, cis-

-. Permethrin, trans-

- Toxaphene

-- $\quad \mathrm{HCH}$,alpha,d6-surrogate\%

-- Biphenyl, 3,5-dichloro surrogate\%

-. Octachlorobiphenyl surrogate \%

-. Set number

-- Sample weight

-- Analytical reference number 
SCHEDULE 2502

Schedule Description: Base-Neutral-Acid (BNA) semivolatile organic compounds in bed sediment analyzed by NWQL using Gas Chromatography/Mass Spectrometry (GC/MS).

Sample Requirements: $500 \mathrm{~g}$ of bed sediment, chilled @ $4 \mathrm{deg} . \mathrm{C}$ (packed in ice)

Container Requirements: $500-\mathrm{mL}$ wide-mouth glass container

Lab. Parameter CAS
code code num CAS

Compound name

MRL

$521200000 \mathrm{~B}$

$521100000 \mathrm{~B}$

$527600000 \mathrm{~B}$

$524500000 \mathrm{~B}$

$524400000 \mathrm{~B}$

$521300000 \mathrm{~B}$

$527900000 \mathrm{~B}$

$521700000 \mathrm{~B}$

523200000 B

528300000 B

$520100000 \mathrm{~B}$

$5234 \quad 00000 \mathrm{~B}$

$522200000 \mathrm{~B}$

$523300000 \mathrm{~B}$

$527200000 \mathrm{~B}$

524700000 B

522800000 B

$5226 \quad 00000 \mathrm{~B}$

522900000 B

$5278 \quad 00000 \mathrm{~B}$

$522500000 \mathrm{~B}$

$525400000 \mathrm{~B}$

527500000 B

$523100000 \mathrm{~B}$

$520800000 \mathrm{~B}$

$520900000 \mathrm{~B}$

521500000 B

$521600000 \mathrm{~B}$

$524000000 \mathrm{~B}$

$5218 \quad 00000 \mathrm{~B}$

$5220 \quad 00000 \mathrm{~B}$

527300000 B

521000000 B

$5230 \quad 00000$ B

524200000 B

$521400000 \mathrm{~B}$

524600000 B

520700000 B

$526700000 \mathrm{~B}$

$526600000 \mathrm{~B}$

$526500000 \mathrm{~B}$

526400000 B

527000000 B

$527400000 \mathrm{~B}$

$521900000 \mathrm{~B}$

$5248 \quad 00000$ B

528200000 B

$528100000 \mathrm{~B}$

527700000 B

$524900000 \mathrm{~B}$

\section{-- Acenaphthylene}

-. Acenaphthene

-- Acridine

-- Amine, n-Nitroso-Di-n-Propyl-

-. Amine, n-Nitroso-Diphenyl-

-- Anthracene

-- Anthracene, 2-Methyl-

-- Benzo[a]anthracene

-- Dibenzo[a,h]anthracene

-- Anthraquinone

.. Benzene, 1,2,4-Trichloro-

-. Benzene, 1,2-Dichloro-

-- Benzene, 1,3-Dichloro-

-- Benzene, 1,4-Dichloro-

- Azobenzene

-- Benzene, Nitro-

-- Benzene, pentachloro-

-- Benzene, Pentachloronitro-

-- Cyclobutadiene, Hexachloro- M-Del

-- 9H-Carbazole

-- Chrysene

-- p-Cresol

-- Dibenzothiophene

-- Ethane, hexachloro-

-- Ether, 4-Bromophenylphenyl-

-- Ether, 4-Chlorophenylphenyl-

-- Ether, bis(2-Chloroethyl)-

- Ether, bis(2-Chloroisopropyl)-

-. Fluoranthene 50

-- Benzo[b]fluoranthene 50

-. Benzo[k]fluoranthene 50

-. 9H-Fluorene, 1-Methyl- 50

-. 9H-Fluorene 50

-- Pentadiene, hexachloro-cyclo M-Del

-- Isophorone 50

-- Methane, bis(2-Chloroethoxy)- 50

- Naphthalene 50

-. Naphthalene, 2-Chloro- 50

-- Naphthalene, 1,2-Dimethyl- 50

-- Naphthalene, 1,6-Dimethyl- $\quad 50$

-- Naphthalene, 2,6-Dimethyl- 50

- Naphthalene, 2-Ethyl- 50

-- Naphthalene, 2,3,6-Trimethyl- 50

-. Pentachloroanisole 50

-- Benzo[g,h,i]perylene- $\quad 50$

-- Phenanthrene 50

-- Phenanthrene, 1-Methyl- 50

-- Phenanthrene, 4,5-Methylene- 50

-- Phenanthridine 50

-- Phenol 50
SCHEDULE 2502-.Continued

Lab. Parameter CAS

code code number compound name $\quad(u g / K g)$

526800000 B $\quad$-- $\quad$ Phenol, 2,4-Dinitro- M-Del

527100000 B -- Phenol, 2-Methyl-4,6-Dinitro- M-Del

526300000 B $\quad$-- $\quad$ Phenol, 2,3,5,6-Tetramethyl- M-Del

525500000 B $\quad$-- $\quad$ Phenol, 2-Nitro- M-Del

526900000 B $\quad$-. $\quad$ Phenol, 4-Nitro- M-Del

520400000 B -- Phenol, 2,4,6-Trichloro- M-Del

525800000 B $\quad$-- $\quad$ Phenol, 3,5-Dimethyl- 50

525900000 B $\quad$-- $\quad$ Phenol, 2,4,6-Trimethyl- M-Del

526200000 B -- Phenol, 4-Chloro-3-Methyl- 50

525700000 B $\quad$-- $\quad$ Phenol, 2,4-Dichloro- M-Del

525600000 B $\quad$-- $\quad$ Phenol, C8-Alkyl- $\quad 50$

522700000 B $\quad$-. $\quad$ Phenol, Pentachloro- M-Del

522300000 B -- $\quad$ Phthalate, bis(2-Ethylhexyl)- 50

522400000 B $\quad$-- $\quad$ Phthalate, Butylbenzyl- 50

523500000 B $\quad--\quad$ Phthalate, Di-n-Butyl- 50

523900000 B $\quad--\quad$ Phthalate, Di-n-Octyl- 50

523700000 B $\quad$-- $\quad$ Phthalate, Diethyl- $\quad 50$

523800000 B $\quad--\quad$ Phthalate, Dimethyl- $\quad 50$

525200000 B $\quad$-. $\quad$ Pyrene 50

528400000 B -- $\quad$ Pyrene, 1-Methyl- 50

522100000 B $\quad$-- $\quad$ Benzo[a]pyrene $\quad 50$

$524100000 \mathrm{~B} \quad--\quad$ Indeno[1,2,3-cd]pyrene $\quad 50$

$528500000 \mathrm{~B} \quad--\quad 2,2-$ Biquinoline $\quad 50$

526000000 B $\quad$-- $\quad$ Quinoline 50

$528000000 \mathrm{~B} \quad--\quad$ Benzo[c]quinoline $\quad 50$

$526100000 \mathrm{~B} \quad$-. $\quad$ Isoquinoline $\quad 50$

$520300000 \mathrm{~B} \quad--\quad$ Toluene, 2,4-Dinitro- $\quad 50$

520500000 B $\quad$-- $\quad$ Toluene, 2,6-Dinitro- 50

528600000 B $\quad$-- $\quad$ Terphenyl, d14-surrogate \%

528700000 B $\quad$-- $\quad$ Benzene, Nitro, d5-, surrogate \%

528800000 B $\quad$-- $\quad$ Biphenyl, 2-Fluoro- surrogate \%

529000000 B $\quad$-- $\quad$ Set number 


\section{SCHEDULE 79}

Schedule Description: Organochlorine herbicides recoverable from whole water by $\mathrm{GC} / \mathrm{ECD}$

Sample Requirements: $800-1,000 \mathrm{~mL}, \mathrm{GCC}$, unfiltered, chilled \& maintained@ 4 deg. C

Container Requirement: 1-L amber, Boston-round pesticide glass bottle, cleaned and burned from NWQL

$\begin{array}{llrlr}\begin{array}{l}\text { Lab. } \\ \text { code }\end{array} & \begin{array}{c}\text { Parameter } \\ \text { code }\end{array} & \begin{array}{l}\text { CAS } \\ \text { number }\end{array} & \text { Compound name } & \begin{array}{r}\text { MRL } \\ \text { (ug/L) }\end{array} \\ 0372 & 39730 \mathrm{~B} & 94757 & 2,4-\mathrm{D} & 0.01 \\ 0373 & 39740 \mathrm{~B} & 93765 & 2,4,5-\mathrm{T} & .01 \\ 0374 & 39760 \mathrm{~B} & 93721 & \text { Silvex } & .01 \\ 0402 & 82183 \mathrm{~A} & 120365 & 2,4-\mathrm{DP} & .01 \\ 0748 & 39720 \mathrm{~A} & 1918021 & \text { Picloram } & .01 \\ 0749 & 82052 \mathrm{~A} & 1918009 & \text { Dicamba } & .01 \\ 1803 & 99859 \mathrm{~A} & & \text { Sample volume, mL, SC 79 } & \\ 1836 & 00000 \mathrm{~A} & & \text { Set number SC 79 } & \end{array}$

\section{SCHEDULE 1301}

Schedule Description: Chlorophenoxy acid herbicides, dissolved, recoverable from whole-water, analyzed by GC/ECD

Sample Requirements: 800-1,000 mL, GCC, unfiltered, chilled \& maintained@4 deg. C

Container Requirement: 1-L amber, Boston-round pesticide glass bottle, cleaned and burned from NWQL

$\begin{array}{llrlr}\begin{array}{l}\text { Lab. } \\ \text { code }\end{array} & \begin{array}{c}\text { Parameter } \\ \text { code }\end{array} & \begin{array}{l}\text { CAS } \\ \text { number }\end{array} & \text { Compound name } & \begin{array}{r}\text { MRL } \\ \text { (Mg/L) }\end{array} \\ & & & & \\ 0477 & 39732 \mathrm{~A} & 94757 & 2,4-\mathrm{D} & 0.01 \\ 0478 & 39742 \mathrm{~A} & 93765 & 2,4,5-\mathrm{t} & .01 \\ 0479 & 39762 \mathrm{~A} & 93721 & \text { Silvex } & .01 \\ 0487 & 82356 \mathrm{~A} & 120365 & 2,4-\mathrm{DP} & .01 \\ 1816 & 00000 \mathrm{~A} & & \text { Sample volume, mL, SC 1301 } \\ 1864 & 00000 \mathrm{~A} & & \text { Set number SC 1301 } & \end{array}$

\section{SCHEDULE 1304}

Schedule Description: Chlorophenoxy acid herbicides recoverable from whole-water, analyed by $G C / E C D$

Sample Requirements: 800-1,000 mL, GCC, unfiltered, chilled \& maintained@ 4 deg. C

Container Requirement: 1-L amber, Boston-round pesticide glass bottle, cleaned and burned from NWQL

$\begin{array}{lcrlr}\begin{array}{l}\text { Lab. } \\ \text { code }\end{array} & \begin{array}{c}\text { Parameter } \\ \text { code }\end{array} & \begin{array}{l}\text { CAS } \\ \text { number }\end{array} & \text { Compound name } & \begin{array}{r}\text { MRL } \\ \text { (Lg/L) }\end{array} \\ & & & & \\ 0372 & 39730 \mathrm{~B} & 94757 & \text { 2,4-D } & 0.01 \\ 0373 & 39740 \mathrm{~B} & 93765 & 2,4,5-\mathrm{T} & .01 \\ 0374 & 39760 \mathrm{~B} & 93721 & \text { Silvex } & .01 \\ 0402 & 82183 \mathrm{~A} & 120365 & \text { 2,4-DP } & .01 \\ 1800 & 00000 \mathrm{~A} & & \text { Sample volume, } \mathrm{mL}, \text { SC } 1304 & \\ 1839 & 00000 \mathrm{~A} & & \text { Set number SC } 1304 & \end{array}$

\section{SCHEDULE 1306}

Schedule Description: DBCP \& EDB recoverable from whole water using hexane, analyzed by GC/ECD

Sample Requirements: $3 \times 40-\mathrm{mL}$ volatile organic vials (GCV), unfiltered, filled to top and capped without bubbles, put into "poly" sleeves or wrapped in bubble wrap, chilled \& maintained @ 4 deg. $\mathrm{C}$

Container Requirement: $3 \times 40-\mathrm{mL}$ VOA vials, with Teflon-lined caps, quality-control, checked and obtained from NWQL

\begin{tabular}{|c|c|c|c|c|}
\hline $\begin{array}{l}\text { Lab. } \\
\text { code }\end{array}$ & $\begin{array}{c}\text { Parameter } \\
\text { code }\end{array}$ & $\begin{array}{l}\text { CAS } \\
\text { number }\end{array}$ & Compound name & $\begin{array}{l}\text { MRL } \\
(\lg / \mathrm{L})\end{array}$ \\
\hline & $82625 \mathrm{D}$ & 96128 & 1,2-Dibromo-3-chloropropane & e 0.03 \\
\hline 77 & $77651 \mathrm{D}$ & 106934 & 1,2-Dibromoethane & .04 \\
\hline
\end{tabular}

\section{SCHEDULE 1307}

Schedule Description: Regulatory volatile compounds, recoverable from whole-water, using the purge and trap tecqhnique and GC MS

Sample Requirements: $3 \times 40-\mathrm{mL}$ volatile organic vials (GCV), unfiltered, filled to top and capped without bubbles, put into "poly" sleeves or wrapped in bubble wrap, chilled \& maintained @ 4 deg. C

Container Requirement: $3 \times 40-\mathrm{mL}$ VOA vials, with Teflon-lined caps, quality-control, checked and obtained from NWQL

Lab. Parameter CAS MRL

code code number Compound name (1ng/L)

$\begin{array}{llrll}1287 & 34030 \text { B } & 71432 & \text { Benzene } & 0.2 \\ 1288 & 32104 \text { B } & 75252 & \text { Bromoform } & .2 \\ 1289 & 32102 \text { B } & 56235 & \text { Carbontetrachloride } & .2 \\ 1290 & 34301 \text { B } & 108907 & \text { Chlorobenzene } & .2 \\ 1291 & 32105 \text { B } & 124481 & \text { Chlorodibromomethane } & .2 \\ 1294 & 32106 \text { B } & 7663 & \text { Chloroform } & .2 \\ 1295 & 32101 \text { B } & 75274 & \text { Dichlorobromomethane } & .2 \\ 1296 & 34668 \text { B } & 75718 & \text { Dichlorodifluoromethane } & .2 \\ 1297 & 34496 \text { B } & 75343 & \text { 1,1-Dichloroethane } & .2 \\ 1298 & 32103 \text { B } & 107062 & \text { 1,2-Dichloroethane } & .2 \\ 1299 & 34501 \text { B } & 75354 & \text { 1,1-Dichlorethylene } & .2 \\ 1300 & 34546 \text { B } & 56605 & \text { 1,2-Transdichloroethene } & .2 \\ 1301 & 34541 \text { B } & 78875 & \text { 1,2-Dichloropropane } & .2 \\ 1303 & 34371 \text { B } & 100414 & \text { Ethylbenzene } & .2 \\ 1305 & 34423 \text { B } & 75092 & \text { Methylenechloride } & .2 \\ 1307 & 34475 \text { B } & 127184 & \text { Tetrachloroethylene } & .2 \\ 1308 & 34010 \text { B } & 108883 & \text { Toluene } & .2 \\ 1309 & 34506 \text { B } & 71556 & \text { 1,1,1-Trichloroethane } & .2 \\ 1311 & 39180 \text { B } & 79016 & \text { Trichloroethylene } & .2 \\ 1312 & 34488 \text { B } & 75694 & \text { Trichlorofluoromethane } & .2 \\ 1313 & 39175 \text { B } & 75014 & \text { Vinyl chloride } & .2 \\ 1314 & 34536 \text { B } & 95501 & \text { 1,2-Dichlorobenzene } & .2 \\ 1315 & 34566 \text { B } & 541731 & \text { 1,3-Dichlorobenzene } & .2 \\ 1316 & 34571 \text { B } & 106467 & \text { 1,4-Dichlorobenzene } & .2 \\ 1328 & 77128 \text { B } & 100425 & \text { Styrene } & .2 \\ 1330 & 81551 \text { B } & 1330207 & \text { Xylene } & .2 \\ 1656 & 77093 \text { A } & 156592 & \text { Cis-1,2-dichloroethene } & .2 \\ 1681 & 77652 \text { A } & 76131 & \text { Trichlorotrifluoroethane } & .5\end{array}$




\section{SCHEDULE 1316}

Schedule Description:Organophosphate pesticides, dissolved, recoverable from filtered water, analyzed by GC/FPD

Sample Requirements: $800-1,000 \mathrm{~mL}, \mathrm{GCC}$, collected unfiltered, but filtered in the lab using precleaned glass-fiber filter, chilled \& maintained@4 deg. C

Container Requirement: 1-L amber, Boston-round pesticide glass botthe, cleaned and burned from NWQL

$\begin{array}{llrlr}\begin{array}{l}\text { Lab. } \\ \text { code }\end{array} & \begin{array}{c}\text { Parameter } \\ \text { code }\end{array} & \begin{array}{l}\text { CAS } \\ \text { number }\end{array} & \begin{array}{r}\text { Compound name } \\ \text { (ug } / \mathrm{L} \text {. }\end{array} \\ 0423 & 39572 \mathrm{~A} & 333415 & \text { Diazinon } & 0.01 \\ 0424 & 82346 \mathrm{~A} & 563122 & \text { Ethion } & .01 \\ 0425 & 39532 \mathrm{~A} & 121755 & \text { Malathion } & .01 \\ 0426 & 39602 \mathrm{~A} & 298000 & \text { Methylparathion } & .01 \\ 0427 & 39542 \mathrm{~A} & 56382 & \text { Parathion } & .01 \\ 0428 & 82342 \mathrm{~A} & 786196 & \text { Trithion } & .01 \\ 1811 & 99867 \mathrm{~A} & & \text { Sample volume, mL, SC 1316 } & 1 \\ 1866 & 00000 \mathrm{~A} & & \text { Set number SC 1316 } & \end{array}$

\section{SCHEDULE 1317}

Schedule Description: Organophosphate pesticides, suspended, recoverable from suspended material, analyzed by GC/FPD

Sample Requirements: 800-1,000 mL, GCC, collected unfiltered, but filtered in the lab using precleaned glass-fiber filter, chilled \& maintained @ 4 deg. C

Container Requirement: 1-L amber, Boston-round pesticide glass bottle, cleaned and burned from NWQL

$\begin{array}{lcrlr}\begin{array}{l}\text { Lab. } \\ \text { code }\end{array} & \begin{array}{c}\text { Parameter } \\ \text { code }\end{array} & \begin{array}{c}\text { CAS } \\ \text { number }\end{array} & \begin{array}{r}\text { MRL } \\ \text { Compound name }\end{array} \\ 0417 & 39573 \text { A } & 333415 \text { Diazinon } \\ 0418 & 82347 \text { A } & 563122 & \text { Ethion } & 0.01 \\ 0419 & 39533 \text { A } & 121755 \text { Malathion } & .01 \\ 0420 & 39603 \text { A } & 298000 & \text { Methylparathion } & .01 \\ 0421 & 39543 \text { A } & 56382 & \text { Parathion } & .01 \\ 0422 & 82343 \text { A } & 786196 & \text { Trithion } & .01 \\ 0485 & 82345 \text { A } & 953173 \text { Methyltrithion } & .01 \\ & & & .01\end{array}$

\section{SCHEDULE 1319}

Schedule Description: Organophosp hate pesticides (total recoverable), where recoverable from whole water, analyzed by GC/FPD

Sample Requirements: 800-1,000 mL, GCC, unfiltered, chilled \& maintained @ 4 deg. C

Container Requirement: 1-L amber, Boston-round pesticide glass bottle, cleaned and burned from NWQL

\begin{tabular}{|c|c|c|c|c|}
\hline $\begin{array}{l}\text { Lab. } \\
\text { code }\end{array}$ & $\begin{array}{c}\text { Parameter } \\
\text { code }\end{array}$ & $\begin{array}{l}\text { CAS } \\
\text { number }\end{array}$ & Compound name & $\begin{array}{l}\text { MRL } \\
(1 \mathrm{~g} / \mathrm{L})\end{array}$ \\
\hline 0378 & $39570 \mathrm{~B}$ & 333415 & Diazinon & 0.01 \\
\hline 0379 & $39398 \mathrm{~B}$ & 563122 & Ethion & .01 \\
\hline 0380 & $39530 \mathrm{~B}$ & 121755 & Malathion & .01 \\
\hline 0381 & $39600 \mathrm{~B}$ & 298000 & Methylparathion & .01 \\
\hline 0383 & $39540 \mathrm{~B}$ & 56382 & Parathion & .01 \\
\hline 0384 & 39786 B & 786196 & Trithion & .01 \\
\hline 0592 & 39011 A & 298044 & Di-Syston (disulfoton) & .01 \\
\hline 0593 & $39023 \mathrm{~A}$ & 298022 & Phorate & .01 \\
\hline 0753 & $38932 \mathrm{~A}$ & 2921882 & Chlorpyrifos & .01 \\
\hline 0802 & $39040 \mathrm{~A}$ & 78488 & DEF & .01 \\
\hline 1336 & $82614 \mathrm{C}$ & 944229 & Fonofos & .01 \\
\hline 1812 & $99868 \mathrm{~A}$ & & Sample volume, mL, SC 1319 & 1 \\
\hline 1837 & $00000 \mathrm{~A}$ & & Set number SC 1319 & \\
\hline
\end{tabular}

01

01

01

.01

\section{SCHEDULE 1321}

Schedule Description: Organochlorine pesticides with PCB's and PCN's, dissolved, recoverable from filtered water, analyzed by GC/ECD

Sample Requirements: $800-1,000 \mathrm{~mL}, \mathrm{GCC}$, collected unfiltered, but filtered in the lab using precleaned glass-fiber filter, chilled \& maintained@4 deg. C)

Container Requirement: 1-L amber, Boston-round pesticide glass bottle, cleaned and burned from NWQL

\begin{tabular}{|c|c|c|c|c|}
\hline $\begin{array}{l}\text { Lab. } \\
\text { code }\end{array}$ & $\begin{array}{c}\text { Parameter } \\
\text { code }\end{array}$ & $\begin{array}{l}\text { CAS } \\
\text { number }\end{array}$ & Compound name & $\begin{array}{l}\text { MRL } \\
(\operatorname{lng} / L)\end{array}$ \\
\hline 0344 & 82348 A & 72560 & Perthane & 0.1 \\
\hline 0345 & $82354 \mathrm{~A}$ & 115297 & Endosulfan & .01 \\
\hline 0463 & $39331 \mathrm{~A}$ & 309002 & Aldrin & .01 \\
\hline 0464 & 39352 A & 57749 & Chlordane & .1 \\
\hline 0465 & $39361 \mathrm{~A}$ & 72548 & DDD & .01 \\
\hline 0466 & 39366 A & 72559 & DDE & .01 \\
\hline 0467 & 39371 A & 50293 & DDT & .01 \\
\hline 0468 & 39381 A & 60571 & Dieldrin & .01 \\
\hline 0469 & 39391 A & 72208 & Endrin & .01 \\
\hline 0470 & 39411 A & 76448 & Heptachlor & .01 \\
\hline 0471 & $39421 \mathrm{~A}$ & 1024573 & Heptachlor epoxide & .01 \\
\hline 0472 & 39341 A & 58899 & Lindane & .01 \\
\hline 0473 & $39401 \mathrm{~A}$ & 8001352 & Toxaphene & 1 \\
\hline 0474 & $39517 \mathrm{~A}$ & 12767792 & PCB's, gross & .1 \\
\hline 0475 & $82360 \mathrm{~A}$ & 25104556 & PCN's, gross & .1 \\
\hline 0476 & $82350 \mathrm{~A}$ & 72435 & Methoxychlor & .01 \\
\hline 0542 & $39756 \mathrm{~A}$ & 2385855 & Mirex & .01 \\
\hline 1810 & $99863 \mathrm{~A}$ & & Sample volume, mL, SC 1321 & 11 \\
\hline 1868 & $00000 \mathrm{~A}$ & & Set number SC 1321 & \\
\hline
\end{tabular}

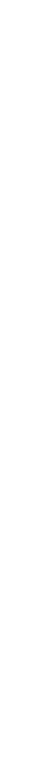




\section{SCHEDULE 1324}

Schedule Description: Onganochlorine pesticides with PCB's and PCN's, total recoverable, and recoverable from whole water, analyzed by GC/ECD

Sample Requirements: 800-1,000 mL, GCC, unfiltered, chilled \& maintained @ 4 deg. C

Container Requirement: 1-L amber, Boston-round pesticide glass bottle, cleaned and burned from NWQL

\begin{tabular}{|c|c|c|c|c|}
\hline $\begin{array}{l}\text { Lab. } \\
\text { code }\end{array}$ & $\begin{array}{c}\text { Parameter } \\
\text { code }\end{array}$ & $\begin{array}{l}\text { CAS } \\
\text { number }\end{array}$ & Compound name & $\begin{array}{l}\text { MRL } \\
(\mathrm{ug} / \mathrm{L})\end{array}$ \\
\hline 0348 & $39034 \mathrm{~A}$ & 72560 & Perthane & 0.1 \\
\hline 0349 & $39388 \mathrm{C}$ & 115297 & Endosulfan I & .01 \\
\hline 0350 & $39330 \mathrm{C}$ & 309002 & Aldrin & .01 \\
\hline 0351 & $39350 \mathrm{~B}$ & 57749 & Chlordane & .1 \\
\hline 0352 & $39360 \mathrm{C}$ & 72548 & DDD & .01 \\
\hline 0353 & $39365 \mathrm{C}$ & 72559 & $\mathrm{DDE}$ & .01 \\
\hline 0354 & $39370 \mathrm{C}$ & 50293 & DDT & .01 \\
\hline 0355 & $39380 \mathrm{C}$ & 60571 & Dieldrin & .01 \\
\hline 0356 & $39390 \mathrm{C}$ & 72208 & Endrin & .01 \\
\hline 0357 & $39410 \mathrm{C}$ & 76448 & Heptachlor & .01 \\
\hline 0358 & $39420 \mathrm{C}$ & 1024573 & Heptachlor epoxide & .01 \\
\hline 0359 & $39340 \mathrm{C}$ & 58899 & Lindane & .01 \\
\hline 0360 & $39400 \mathrm{~B}$ & 8001352 & Toxaphene & 1 \\
\hline 0392 & $39516 \mathrm{~B}$ & 12767792 & PCB's, gross & .1 \\
\hline 0393 & $39250 \mathrm{~B}$ & 25104556 & PCN's, gross & .1 \\
\hline 0400 & $39480 \mathrm{~B}$ & 72435 & Methoxychlor & .01 \\
\hline 0544 & $39755 \mathrm{~B}$ & 2385855 & Mirex & .01 \\
\hline 1808 & $99865 \mathrm{~A}$ & & $\begin{array}{l}\text { Sample volume, } \mathrm{mL} \text {, } \\
\text { SC } 1324\end{array}$ & 1 \\
\hline 1840 & $00000 \mathrm{~A}$ & & Set number SC 1324 & \\
\hline
\end{tabular}

\section{SCHEDULE 1331}

Schedule Description: Organochlorine pesticides with PCB's and PCN's, dissolved, recoverable from filtered water, analyzed by GC/ECD

Sample Requirements: 800-1,000 mL, GCC, collected unfiltered, but filtered in the lab using precleaned glass-fiber filter, chilled \& maintained@4 deg.C

Container Requirement: 1-L amber, Boston-round pesticide glass bottle, cleaned and burned from NWQL

\begin{tabular}{|c|c|c|c|c|}
\hline $\begin{array}{l}\text { Lab. } \\
\text { code }\end{array}$ & $\begin{array}{c}\text { Parameter } \\
\text { code }\end{array}$ & $\begin{array}{l}\text { CAS } \\
\text { number }\end{array}$ & Compound name & $\begin{array}{l}\mathrm{MRL} \\
(\mu \mathrm{g} / \mathrm{L})\end{array}$ \\
\hline 0344 & $82348 \mathrm{~A}$ & 72560 & Perthane & 0.1 \\
\hline 0345 & 82354 A & 115297 & Endosulfan & .01 \\
\hline 0423 & 39572 A & 333415 & Diazinon & 0 \\
\hline 0424 & $82346 \mathrm{~A}$ & 563122 & Ethion & .01 \\
\hline 0425 & 39532 A & 121755 & Malathion & 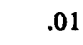 \\
\hline 0426 & 39602 A & 298000 & Methylparathion & 01 \\
\hline 0427 & 39542 A & 56382 & Parathion & 01 \\
\hline 0428 & $82342 \mathrm{~A}$ & 786196 & Trithion & 1 \\
\hline 0463 & 39331 A & 309002 & Aldrin & 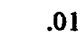 \\
\hline 0464 & $39352 \mathrm{~A}$ & 57749 & Chlordane & .1 \\
\hline 0465 & $39361 \mathrm{~A}$ & 72548 & DDD & \\
\hline 0466 & $39366 \mathrm{~A}$ & 72559 & DDE & \\
\hline 0467 & 39371 A & 50293 & DDT & .01 \\
\hline 0468 & 39381 A & 60571 & Dieldrin & 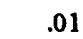 \\
\hline 0469 & 39391 A & 72208 & Endrin & \\
\hline 0470 & 39411 A & 76448 & Heptachlor & \\
\hline 0471 & $39421 \mathrm{~A}$ & 1024573 & Heptachlor epoxide & \\
\hline 0472 & $39341 \mathrm{~A}$ & 58899 & Lindane & \\
\hline 0473 & 39401 A & 8001352 & Toxaphene & 1 \\
\hline 0474 & 39517 A & 12767792 & PCB's, gross & .1 \\
\hline 0475 & $82360 \mathrm{~A}$ & 25104556 & PCN's, gross & .1 \\
\hline 0476 & $82350 \mathrm{~A}$ & 72435 & Methoxychlor & \\
\hline 0542 & 39756 A & 2385855 & Mirex & 0 \\
\hline
\end{tabular}




\section{SCHEDULE 1334}

Schedule Description: Organochlorine and organophosphate pesticides, recoverable from whole water

Sample Requirements: 800-1,000 mL, GCC, unfiltered, chilled \& maintained @ 4deg. C

Container Requirement: 2x1-L amber, Boston-round pesticide glass bottle, cleaned and burned from NWQL

\begin{tabular}{|c|c|c|c|}
\hline $\begin{array}{l}\text { Lab. } \\
\text { code }\end{array}$ & $\begin{array}{c}\text { Parameter } \\
\text { code }\end{array}$ & $\begin{array}{l}\text { CAS } \\
\text { number }\end{array}$ & Compound name \\
\hline 0348 & 39034 A & 72560 & Perthane \\
\hline 0349 & $39388 \mathrm{C}$ & 115297 & Endosulfan I \\
\hline 0350 & $39330 \mathrm{C}$ & 309002 & Aldrin \\
\hline 0351 & $39350 \mathrm{~B}$ & 57749 & Chlordane \\
\hline 0352 & $39360 \mathrm{C}$ & 72548 & DDD \\
\hline 0353 & $39365 \mathrm{C}$ & 72559 & DDE \\
\hline 0354 & $39370 \mathrm{C}$ & 50293 & DDT \\
\hline 0355 & $39380 \mathrm{C}$ & 60571 & Dieldrin \\
\hline 0356 & $39390 \mathrm{C}$ & 72208 & Endrin \\
\hline 0357 & $39410 \mathrm{C}$ & 76448 & Heptachlor \\
\hline 0358 & $39420 \mathrm{C}$ & 1024573 & Heptachlor epoxide \\
\hline 0359 & $39340 \mathrm{C}$ & 58899 & Lindane \\
\hline 0360 & $39400 \mathrm{~B}$ & 8001352 & Toxaphene \\
\hline 0378 & $39570 \mathrm{~B}$ & 333415 & Diazinon \\
\hline 0379 & $39398 \mathrm{~B}$ & 563122 & Ethion \\
\hline 0380 & $39530 \mathrm{~B}$ & 121755 & Malathion \\
\hline 0381 & $39600 \mathrm{~B}$ & 298000 & Methylparathion \\
\hline 0383 & $39540 \mathrm{~B}$ & 56382 & Parathion \\
\hline 0384 & $39786 \mathrm{~B}$ & 786196 & Trithion \\
\hline 0392 & $39516 \mathrm{~B}$ & 12767792 & PCB's, gross \\
\hline 0393 & $39250 \mathrm{~B}$ & 25104556 & PCN's, gross \\
\hline 0400 & $39480 \mathrm{~B}$ & 72435 & Methoxychlor \\
\hline 0544 & $39755 \mathrm{~B}$ & 2385855 & Mirex \\
\hline 0592 & 39011 A & 298044 & Di-syston (disulfoton) \\
\hline 0593 & 39023 A & 298022 & Phorate \\
\hline 0753 & 38932 A & 2921882 & Chlorpyrifos \\
\hline 0802 & $39040 \mathrm{~A}$ & 78488 & DEF \\
\hline 1336 & $82614 \mathrm{C}$ & 944229 & Fonofos \\
\hline
\end{tabular}

MRL $(\operatorname{Lg} / \mathrm{L})$

0.10

.01

.01

.10

.01

.01

.01

.01

.01

.01

.01

.01

1

.01

.01

.01

.01

.01

.01

.10

.10

.01

.01

.01

.01

.01

.01

.01

\section{SCHEDULE 1359}

Schedule Description: Carbamate pesticides, recoverable, whole water Sample Requirements: 800-1,000 mL, GCC, unfiltered, chilled \& maintained@4 deg. C

Container Requirement: 1-L amber, Boston-round pesticide glass bottle, cleaned and burned from NWQL

\begin{tabular}{|c|c|c|c|c|}
\hline $\begin{array}{l}\text { Lab. } \\
\text { code }\end{array}$ & $\begin{array}{l}\text { Parameter } \\
\text { code }\end{array}$ & $\begin{array}{l}\text { CAS } \\
\text { number }\end{array}$ & Compound name & $\begin{array}{c}\text { MRL } \\
\text { (ug/h) }\end{array}$ \\
\hline 0636 & $39750 \mathrm{~A}$ & 63252 & Carbaryl & -- \\
\hline 0637 & $39052 \mathrm{~A}$ & 122429 & Propham & -- \\
\hline 0638 & 39051 A & 16752775 & Methomyl & -- \\
\hline 1335 & $82613 \mathrm{C}$ & 23135220 & Oxamyl & - \\
\hline 1337 & $82615 \mathrm{C}$ & 1563662 & Carbofuran & -- \\
\hline 1338 & $82619 \mathrm{C}$ & 116063 & Aldicarb & -- \\
\hline 1343 & $82586 \mathrm{C}$ & 1646873 & Aldicarb Sulfoxide & - \\
\hline 1344 & $82587 \mathrm{C}$ & 1646884 & Aldicarb Sulfone & -- \\
\hline 1351 & 77441 A & 90153 & 1-Naphthol & -- \\
\hline 1353 & 82584 A & 16655826 & Hydroxycarbofuran & - \\
\hline 1448 & 30296 A & 114261 & Propoxur & -- \\
\hline 1449 & $30282 \mathrm{~A}$ & 2032657 & Methiocarb & -- \\
\hline 1813 & 99869 A & & Sample volume, $\mathrm{mL}$ & 9 \\
\hline
\end{tabular}

\section{SCHEDULE 1361}

Schedule Description: Individual Aroclor PCB's, dissolved, recoverable from filtered water

Sample Requirements: 800-1,000 mL, GCC, collected unfiltered, but filtered in the lab using precleaned glass-fiber filter, chilled \& maintained@4 deg.C

Container Requirement: 1-L amber, Boston-round pesticide glass bottle, cleaned and burned from NWQL

$\begin{array}{lcllr}\begin{array}{l}\text { Lab. } \\ \text { code }\end{array} & \begin{array}{c}\text { Parameter } \\ \text { code }\end{array} & \begin{array}{c}\text { CAS } \\ \text { number }\end{array} & \begin{array}{r}\text { Compound name } \\ \text { (ug/L) }\end{array} \\ & & & & \\ 0763 & 39509 \text { A } & 11096825 & \text { Aroclor } 1260 & 0.1 \\ 0767 & 39505 \text { A } & 11097691 & \text { Aroclor } 1254 & .1 \\ 0771 & 39501 \text { A } & 12672296 & \text { Aroclor } 1248 & .1 \\ 0775 & 34457 \text { A } & 53469219 & \text { Aroclor 1242 } & .1 \\ 0779 & 34665 \text { A } & 11141165 & \text { Aroclor 1232 } & .1 \\ 0783 & 34662 \text { A } & 11104282 & \text { Aroclor 1221 } & .1 \\ 0787 & 34672 \text { A } & 12674112 & \text { Aroclor 1016 } & .1 \\ 1802 & 00000 \text { A } & \text { Sample volume, mL, SC 1361 } & 1\end{array}$

\section{SCHEDULE 1364}

Schedule Description: Individual Aroclor PCB's, total recoverable, whole water

Sample Requirements: 800-1,000 mL, GCC, unfiltered, chilled \& maintained @4 deg. C

Container Requirement: 1-L amber, Boston-round pesticide glass bottle, cleaned and burned from NWQL

$\begin{array}{llllr}\begin{array}{l}\text { Lab. } \\ \text { code }\end{array} \begin{array}{llll}\text { Parameter } \\ \text { code }\end{array} & \begin{array}{l}\text { CAS } \\ \text { number }\end{array} & \begin{array}{r}\text { MRL } \\ (\mu \mathrm{g} / \mathrm{L} \text { ) }\end{array} \\ 0809 & 34671 \text { B } & 12674112 & \text { Aroclor 1016 } & \\ 0810 & 39488 \text { B } & 11104282 & \text { Aroclor 1221 } & 0.1 \\ 0811 & 39492 \text { B } & 11141165 & \text { Aroclor 1232 } & .1 \\ 0812 & 39496 \text { B } & 53469219 & \text { Aroclor 1242 } & .1 \\ 0813 & 39500 \text { B } & 12672296 & \text { Aroclor 1248 } & .1 \\ 0814 & 39504 \text { B } & 11097691 & \text { Aroclor 1254 } & .1 \\ 0815 & 39508 \text { B } & 11096825 & \text { Aroclor 1260 } & .1 \\ 1801 & 00000 \text { A } & & \text { Sample volume, mL, SC 1364 } & 1 \\ 1871 & 00000 \text { A } & & \text { Set number SC 1364 } & .1\end{array}$

\section{SCHEDULE 1378}

Schedule Description: BETX compounds, recoverable from whole water

Sample Requirements: $3 \times 40-\mathrm{mL}$ volatile organic vials (GCV), unfiltered, filled to top and capped without bubbles, put into "poly" sleeves or wrapped in bubble wrap, chilled \& maintained @ 4 deg. C

Container Requirement: $3 \times 40-\mathrm{mL}$ VOA vials, with Teflon-lined caps, quality-control, checked and obtained from NWQL

\begin{tabular}{llrlr}
$\begin{array}{l}\text { Lab. Parameter } \\
\text { code }\end{array}$ code & \multicolumn{1}{c}{$\begin{array}{c}\text { CAS } \\
\text { number }\end{array}$} & $\begin{array}{r}\text { MRL } \\
\text { Compound name }\end{array}$ \\
1287 & 34030 B & 71432 Benzene & B \\
1303 & 34371 B & 100414 Ethylbenzene & 0.2 \\
1308 & 34010 B & 108883 Toluene & .2 \\
1330 & 81551 B & 1330207 Xylenes (O,M,P) & .2 \\
1709 & 77135 B & 95476 Xylene ortho & .2 \\
1712 & 85795 B & 108383 Xylene meta and para & .2 \\
& & &
\end{tabular}


SCHEDULE 1379

Schedule Description: Organonitrogen pesticides, total recoverable, using SPE technology

Sample Requirements: $125 \mathrm{~mL}, \mathrm{GCC}$, filtered

Container Requirement: 1x125-mL amber, glass bottle from NWQL

Lab. Parameter CAS

code code number Compound name (ug/L)

158746342 A 15972608 Alachlor $\quad 0.05$

158838401 A 834128 Ametryn

158939632 A 1912249 Atrazine $\quad .05$

159004041 A 21725462 Cyanazine $\quad .20$

159104040 A 6190654 De-sethylatrazine $\quad .05$

159204038 A 1007289 De-sisopropylatrazine $\quad .05$

159339415 A 51218452 Metolachlor

159482630 A 21087649 Metribuzin

159538535 A 139402 Propazine 05

159604035 A 122349 Simazine

159704037 A 1610180 Prometon

159804036 A 7287196 Prometryn 05

$180499860 \mathrm{~A} \quad$ Sample volume, $\mathrm{mL}, \mathrm{SC} 13791$

$\begin{array}{llll}184100000 \mathrm{~A} & \text { Set number SC } 1379 & 0\end{array}$

SCHEDULE 1380

Schedule Description: VOC, total recoverable, using purge and trap and GC/MS

Sample Requirements: $3 \times 40-\mathrm{mL}$ volatile organic vials (GCV), unfi1tered, filled to top and capped without bubbles, put into "poly" sleeves or wrapped in bubble wrap, chilled \& maintained @ 4 deg. $\mathrm{C}$

Container Requirement: $3 \times 40-\mathrm{mL}$ VOA vials, with Teflon-lined caps, quality-control, checked and obtained from NWQL

\begin{tabular}{|c|c|c|c|c|}
\hline $\begin{array}{l}\text { Lab. } \\
\text { code }\end{array}$ & $\begin{array}{c}\text { Parameter } \\
\text { code }\end{array}$ & $\begin{array}{l}\text { CAS } \\
\text { number }\end{array}$ & Compound name & $\begin{array}{l}\text { MRL } \\
(\mathrm{LQ} / \mathrm{L})\end{array}$ \\
\hline 1009 & $30217 \mathrm{~B}$ & 74953 & Dibromomethane & 0.2 \\
\hline 1287 & $34030 \mathrm{~B}$ & 71432 & Benzene & .2 \\
\hline 1288 & $32104 \mathrm{~B}$ & 75252 & Bromoform & .2 \\
\hline 1289 & $32102 \mathrm{~B}$ & 56235 & Carbontetrachloride & 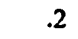 \\
\hline 1290 & $34301 \mathrm{~B}$ & 108907 & Chlorobenzene & .2 \\
\hline 1291 & $32105 \mathrm{~B}$ & 124481 & Dibromochloromethane & .2 \\
\hline 1292 & $34311 \mathrm{~B}$ & 75003 & Chloroethane & .2 \\
\hline 1294 & $32106 \mathrm{~B}$ & 67663 & Chloroform & $?$ \\
\hline 1295 & $32101 \mathrm{~B}$ & 75274 & Bromodichloromethane & .2 \\
\hline 1296 & $34668 \mathrm{~B}$ & 75718 & Dichlorodifluoromethane & .2 \\
\hline 1297 & 34496 B & 75343 & 1,1-Dichloroethane & .2 \\
\hline 1298 & $32103 \mathrm{~B}$ & 107062 & 1,2-Dichloroethane & .2 \\
\hline 1299 & $34501 \mathrm{~B}$ & 75354 & 1,1-Dichloroethene & .2 \\
\hline 1300 & $34546 \mathrm{~B}$ & 156605 & Trans 1,2-dichloroethene & .2 \\
\hline 1301 & $34541 \mathrm{~B}$ & 78875 & 1,2-Dichloropropane & .2 \\
\hline 1303 & $34371 \mathrm{~B}$ & 100414 & Ethylbenzene & .2 \\
\hline 1304 & $34413 \mathrm{~B}$ & 74839 & Bromomethane & .2 \\
\hline 1305 & 34423 B & 75092 & Methylene chloride & .2 \\
\hline 1306 & $34516 \mathrm{~B}$ & 79345 & 1,1,2,2-Tetrachloroethane & .2 \\
\hline 1307 & 34475 B & 127184 & Tetrachloroethene & .2 \\
\hline 1308 & $34010 \mathrm{~B}$ & 108883 & Toluene & .2 \\
\hline 1309 & 34506 B & 71556 & 1,1,1-Trichloroethane & .2 \\
\hline 1310 & $34511 \mathrm{~B}$ & 79005 & 1,1,2-Trichloroethane & .2 \\
\hline 1311 & $39180 \mathrm{~B}$ & 79016 & Trichloroethene & .2 \\
\hline 1312 & 34488 B & 75694 & Trichlorofluoromethane & .2 \\
\hline 1313 & $39175 \mathrm{~B}$ & 75014 & Vinyl chloride & .2 \\
\hline 1315 & $34566 \mathrm{~B}$ & 541731 & 1,3-Dichlorobenzene & 0.2 \\
\hline 1316 & $34571 \mathrm{~B}$ & 106467 & 1,4-Dichlorobenzene & .2 \\
\hline
\end{tabular}

SCHEDULE 1380--Continued

Lab. Parameter CAS MRL

code code number Compound name (ug/L)

131777651 B 1069341,2-Dibromoethane.2

$\begin{array}{lllll}1318 & 34418 \text { B } & 74873 & \text { Chloromethane } & .2\end{array}$

$\begin{array}{lllll}1320 & 34536 \mathrm{C} & 95501 & 1,2-D i c h l o r o b e n z e n e\end{array}$

132634704 B 10061015 Cis-1,3-dichloropropene $\quad .2$

132734699 B 10061026 Trans 1,3-dichloropropene $\quad .2$

$\begin{array}{llll}1328 & 77128 \text { B } & 100425 & \text { Styrene }\end{array}$

$\begin{array}{llllll}1330 & 81551 \text { B } & 1330207 & \text { Xylenes (O,M,P) }\end{array}$

135482625 B 96128 1,2-Dibromo-3-chloropropane 1

147877168 B 563586 1,1-Dichloropropene $\quad .2$

147977170 B 594207 2,2-Dichloropropane $\quad .2$

$\begin{array}{llllll}1480 & 77173 \text { B } & 142289 & \text { 1,3-Dichloropropane } & .2\end{array}$

$\begin{array}{lllll}1481 & 77275 & \mathrm{~B} & 95498 & \text { 2-Chlorotoluene }\end{array}$

148277277 B 106434 4-Chlorotoluene

$\begin{array}{llllll}1483 & 77443 \text { B } & 96184 & 1,2,3-T r i c h l o r o p r o p a n e & .2\end{array}$

148477562 B 630206 1,1,1,2-Tetrachloroethane $\quad .2$

$\begin{array}{lllll}1650 & 34210 \mathrm{~A} & 107028 \text { Acrolein } & 20\end{array}$

165134215 A 107131 Acrylonitrile 20

165278032 A 1634044 Methyltertbutylether 1

165477297 A 74975 Bromochloromethane

165677093 A 156592 Cis-1,2-dichloroethene 2

$\begin{array}{llll}1658 & 34576 \mathrm{C} & 110758 & \text { 2-Chloroethylvinylether } \quad 1\end{array}$

$\begin{array}{lllll}1659 & 77223 & \text { A } & 98828 & \text { Isopropylbenzene }\end{array}$

$\begin{array}{lllll}1661 & 77224 & \text { A } & 103651 & \text { n-Propylbenzene }\end{array}$

166377353 A 98066 Tert-butylbenzene $\quad .2$

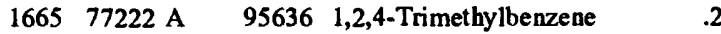

$166777350 \mathrm{~A} \quad 135988$ Sec-butylbenzene

166977356 A 99876 p-Isopropyltoluene

$\begin{array}{llll}1671 & 77342 \mathrm{~A} & 104518 & \mathrm{n} \text {-Butylbenzene }\end{array}$

$\begin{array}{lllll}1673 & 34551 \mathrm{C} & 120821 & 1,2,4-T r i c h l o r o b e n z e n e & .2\end{array}$

$167539702 \mathrm{C} \quad 87683$ Hexachlorobutadiene $\quad .2$

$167734696 \mathrm{C} \quad 91203$ Naphthalene

$167977613 \mathrm{~A} \quad 87616$ 1,2,3-Trichlorobenzene $\quad .2$

$\begin{array}{lllll}1681 & 77652 \mathrm{~A} \quad 76131 & 1,1,2 \text {-Trichloro 1,2,2-trifluoro } .5\end{array}$

$\begin{array}{lllll}1683 & 77226 \text { A } & 108678 & 1,3,5-T r i m e t h y l b e n z e n e & .2\end{array}$

169881555 A 108861 Bromobenzene

\section{SCHEDULE 1381}

Schedule Description: Organic compound profile, total recoverable, methylene chloride extraction, analyzed by GC/FID

Sample Requirements: 800-1,000 mL, GCC, unfiltered, chilled \& maintained @ 4 deg. C

Container Requirement: 1-L amber, Boston-round pesticide glass bottle, cleaned and burned from NWQL

$\begin{array}{lccc}\begin{array}{l}\text { Lab. Parameter } \\ \text { code } \\ \text { code }\end{array} & \begin{array}{l}\text { CAS } \\ \text { number }\end{array} & \begin{array}{c}\text { MRL } \\ \text { Compound name }\end{array} \\ 124099478 \mathrm{~A} & & \mathrm{H}_{2} \mathrm{O} \text { GC/FID profile }\end{array}$


SCHEDULE 1383

Schedule Description: Organic compounds, acid and base/neutral, total recoverable, whole water, methylene chloride and GC/MS

Sample Requirements: $800-1,000 \mathrm{~mL}, \mathrm{GCC}$, unfiltered, chilled \& maintained@ 4 deg. C

Container Requirement: 1-L amber, Boston-round pesticide glass bottle, cleaned and burned from NWQL

\begin{tabular}{|c|c|c|c|c|}
\hline $\begin{array}{l}\text { Lab. } \\
\text { code }\end{array}$ & $\begin{array}{c}\text { Parameter } \\
\text { code }\end{array}$ & $\begin{array}{l}\text { CAS } \\
\text { number }\end{array}$ & Compoun & 0 \\
\hline 055 & 34452 A & 59507 & 4-Chloro-3-methylphenol & \\
\hline 1056 & 34586 A & 95578 & 2-Chloro & \\
\hline 1057 & $34601 \mathrm{~A}$ & 120832 & 2,4-Dichlorophenol & \\
\hline 1058 & $34621 \mathrm{~A}$ & 88062 & 2,4,6-Trichlorophenol & \\
\hline 1059 & 34606 A & 105679 & 2,4-Dimethylphenol & \\
\hline 060 & 34657 A & 534521 & 4,6-Dinitro-2-methylphenol & \\
\hline 1061 & $34616 \mathrm{~A}$ & 51285 & 2,4-Dinitrophenol & \\
\hline 1062 & 34591 A & 88755 & 2-Nitrophenol & \\
\hline 1063 & 34646 A & 100027 & 4-Nitrophenol & 10 \\
\hline 1064 & 39032 A & 87865 & Pentachl & \\
\hline 1065 & 34694 A & 108952 & Phenol & \\
\hline 1066 & $34205 \mathrm{~A}$ & 83329 & Acenaph & \\
\hline 1067 & $34200 \mathrm{~A}$ & 208968 & Acenaph & \\
\hline 1068 & $34220 \mathrm{~A}$ & 120127 & Anthr & \\
\hline 1069 & $39120 \mathrm{~A}$ & 92875 & Benzid & \\
\hline 1070 & $34526 \mathrm{~A}$ & 56553 & Benzo & \\
\hline 1071 & $34230 \mathrm{~A}$ & 205992 & Benz & \\
\hline 1072 & 34242 A & 207089 & Benzo & \\
\hline 1073 & 34247 A & 50328 & Benzola & \\
\hline 1074 & 34521 A & 191242 & Benzo[g,h & 0 \\
\hline 1075 & 34292 A & 85687 & Buty & \\
\hline 1076 & $34278 \mathrm{~A}$ & 111911 & Bis & \\
\hline 1077 & 34273 A & 111444 & Bis & \\
\hline 1078 & 34283 A & 108601 & Bis & \\
\hline 1079 & $34636 \mathrm{~A}$ & 101553 & 4-Br & \\
\hline 1080 & 34581 A & 91587 & $2-\mathrm{Cl}$ & \\
\hline 1081 & $34641 \mathrm{~B}$ & 7005723 & 4-Ch & \\
\hline 1082 & $34320 \mathrm{~A}$ & 218019 & $\mathrm{Chr}$ & \\
\hline 1083 & 34556 A & 53703 & 1,2 & \\
\hline 1084 & $39110 \mathrm{~A}$ & 84742 & $\mathrm{Di}-\mathrm{r}$ & \\
\hline 1085 & 34536 A & 95501 & & \\
\hline 1086 & 34566 A & 541731 & & \\
\hline 1087 & 34571 A & 106467 & & \\
\hline 1088 & $34631 \mathrm{~A}$ & 91941 & & \\
\hline 1089 & 34336 A & 84662 & & \\
\hline 1090 & 34341 A & 131113 & $\mathrm{Di}$ & \\
\hline 1091 & 34611 A & 121142 & $2,4-I$ & \\
\hline 1092 & 34626 A & 606202 & $2,6-I$ & \\
\hline 1093 & $34596 \mathrm{~A}$ & 117840 & $\mathrm{Di}-\mathrm{n}$ & \\
\hline 1094 & $39100 \mathrm{~A}$ & 117817 & Bis & \\
\hline 1095 & 34381 A & 86737 & Fluc & \\
\hline 1096 & 34376 A & 206440 & Fluo & \\
\hline 1097 & $39700 \mathrm{~A}$ & 118741 & Hex & \\
\hline 1098 & 34391 A & 87683 & & \\
\hline 1099 & 34386 A & 77474 & Hexa & \\
\hline 1101 & 34403 A & 193395 & 2,3-cd) pyrene & 10 \\
\hline 1102 & 34408 A & 78591 & Isop & \\
\hline 1103 & 34696 A & 91203 & Nap & \\
\hline 1104 & 34447 A & 98953 & Nitr & \\
\hline 110 & 34438 A & 627 & $\mathrm{n}-\mathrm{N}$ & \\
\hline 1106 & 34433 A & 86306 & n-Nitrosodiphenylamine & \\
\hline 1107 & 34428 A & 621647 & n-Nitrosodi-n-propylamin & \\
\hline 1108 & 34461 A & 85018 & Phenanthrene & \\
\hline
\end{tabular}

20

20 30 10

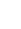
(1)

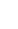
10

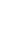
5

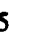

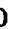

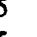

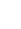

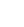

5

5

SCHEDULE 1383-Continued

Lab. Parameter CA

code code number compound name

MRL

110934469 A 129000 Pyrene

$111134551 \mathrm{~A}$

120821 1,2,4-Trichlorobenzene

(1) $/ \mathrm{h})$

169782626 A

$181499855 \mathrm{~A}$ Sample volume 1383/1385 
SCHEDULE 1385

Schedule Description: Organic compounds, acid and base/neutral, total recoverable, whole water, methylene chloride and GC/MS, with unknowns tentatively identified

Sample Requirements: $800-1,000 \mathrm{~mL}$, GCC, unfiltered, chilled \& maintained@ 4 deg. C

Container Requirement: 1- $\mathrm{L}$ amber, Boston-round pesticide glass bottle, cleaned and burned from NWQL

\begin{tabular}{|c|c|c|c|c|}
\hline $\begin{array}{l}\text { Lab. } 1 \\
\text { code }\end{array}$ & $\begin{array}{c}\text { Parameter } \\
\text { code }\end{array}$ & $\begin{array}{l}\text { CAS } \\
\text { number }\end{array}$ & Compound name & $\begin{array}{l}\text { MRL } \\
(\mathrm{u} / \mathrm{L})\end{array}$ \\
\hline 1055 & 34452 A & 59507 & 4-Chloro-3-methylphenol & 30 \\
\hline 1056 & 34586 A & 95578 & 2-Chlorophenol & 5 \\
\hline 1057 & 34601 A & 120832 & 2,4-Dichlorophenol & 5 \\
\hline 1058 & 34621 A & 88062 & 2,4,6-Trichlorophenol & 20 \\
\hline 1059 & $34606 \mathrm{~A}$ & 105679 & 2,4-Dimethylphenol & 5 \\
\hline 1060 & 34657 A & 534521 & 4,6-Dinitro-2-methylphenol & 30 \\
\hline 1061 & 34616 A & 51285 & 2,4-Dinitrophenol & 20 \\
\hline 1062 & 34591 A & 88755 & 2-Nitrophenol & 5 \\
\hline 1063 & 34646 A & 100027 & 4-Nitrophenol & 30 \\
\hline 1064 & 39032 A & 87865 & Pentachlorophenol & 30 \\
\hline 1065 & 34694 A & 108952 & Phenol & 5 \\
\hline 1066 & $34205 \mathrm{~A}$ & 83329 & Acenaphthene & 5 \\
\hline 1067 & $34200 \mathrm{~A}$ & 208968 & Acenaphthylene & 5 \\
\hline 1068 & 34220 A & 120127 & Anthracene & 5 \\
\hline 1069 & $39120 \mathrm{~A}$ & 92875 & Benzidine & 40 \\
\hline 1070 & 34526 A & 56553 & Benzo[a]anthracene & 10 \\
\hline 1071 & $34230 \mathrm{~A}$ & 205992 & Benzo[b]fluoranthene & 10 \\
\hline 1072 & $34242 \mathrm{~A}$ & 207089 & Benzo[k]fluoranthene & 10 \\
\hline 1073 & 34247 A & 50328 & Benzo[a]pyrene & 10 \\
\hline 1074 & $34521 \mathrm{~A}$ & 191242 & Benzo[g,h,i]perylene & 10 \\
\hline 1075 & 34292 A & 85687 & Butyl benzyl phthalate & 5 \\
\hline 1076 & $34278 \mathrm{~A}$ & 111911 & Bis (2-chloroethoxy) methane & 5 \\
\hline 1077 & 34273 A & 111444 & Bis (2-chlorethyl) ether & 5 \\
\hline 1078 & 34283 A & 108601 & Bis (2-chlorisopropyl) ether & 5 \\
\hline 1079 & 34636 A & 101553 & 4-Bromophenylphenyether & 5 \\
\hline 1080 & 34581 A & 91587 & 2-Chloronaphthalene & 5 \\
\hline 1081 & 34641 B & 7005723 & 4-Chlorophenylphenyether & 5 \\
\hline 1082 & $34320 \mathrm{~A}$ & 218019 & Chrysene & 10 \\
\hline 1083 & 34556 A & 53703 & 1,2,5,6-Dibenz [a,h]anthracene & 10 \\
\hline 1084 & $39110 \mathrm{~A}$ & 84742 & Di-n-butyl phthalate & 5 \\
\hline 1085 & $34536 \mathrm{~A}$ & 95501 & 1,2-Dichlorobenzene & 5 \\
\hline 1086 & 34566 A & 541731 & 1,3-Dichlorobenzene & 5 \\
\hline 1087 & 34571 A & 106467 & 1,4-Dichlorobenzene & 5 \\
\hline 1088 & 34631 A & 91941 & 3,3-Dichlorobenzidine & 20 \\
\hline 1089 & 34336 A & 84662 & Diethyl phthalate & 5 \\
\hline 1090 & 34341 A & 131113 & Dimethyl phthalate & 5 \\
\hline 1091 & 34611 A & 121142 & 2,4-Dinitrotoluene & 5 \\
\hline 1092 & 34626 A & 606202 & 2,6-Dinitrotoluene & 5 \\
\hline 1093 & 34596 A & 117840 & Di-n-octyl phthalate & 10 \\
\hline 1094 & $39100 \mathrm{~A}$ & 117817 & Bis (2-ethlyhexyl) phthalate & 5 \\
\hline 1095 & 34381 A & 86737 & Fluorene & 5 \\
\hline 1096 & 34376 A & 206440 & Fluoranthene & 5 \\
\hline 1097 & $39700 \mathrm{~A}$ & 118741 & Hexachlorobenzene & 5 \\
\hline 1098 & 34391 A & 87683 & Hexachlorobutadiene & 5 \\
\hline 1099 & 34386 A & 77474 & Hexachlorocyclopentadiene & 5 \\
\hline 1100 & 34396 A & 67721 & Hexachloroethane & 5 \\
\hline 1101 & 34403 A & 193395 & Indeno $(1,2,3-c d)$ pyrene & 10 \\
\hline 1102 & 34408 A & 78591 & Isophorone & 5 \\
\hline 1103 & 34696 A & 91203 & Naphthalene & 5 \\
\hline 1104 & 34447 A & 98953 & Nitrobenzene & 5 \\
\hline 1105 & 34438 A & 62759 & n-Nitrosodimethlyamine & 5 \\
\hline 1106 & 34433 A & 86306 & n-Nitrosodiphenylamine & 5 \\
\hline
\end{tabular}

\begin{tabular}{|c|c|c|c|c|}
\hline $\begin{array}{l}\text { Lab. } \\
\text { code }\end{array}$ & $\begin{array}{l}\text { DULE 13 } \\
\text { Parameter } \\
\text { code }\end{array}$ & $\begin{array}{l}\text { CAS } \\
\text { number }\end{array}$ & Compound name & $\begin{array}{l}\text { MRL } \\
\text { (Ug/L) }\end{array}$ \\
\hline 1107 & 34428 A & 621647 & n-Nitrosodi-n-propylamine & 5 \\
\hline 1108 & 34461 A & 85018 & Phenanthrene & 5 \\
\hline 1109 & 34469 A & 129000 & Pyrene & 5 \\
\hline 1111 & 34551 A & 120821 & 1,2,4-Trichlorobenzene & 5 \\
\hline 1697 & $82626 \mathrm{~A}$ & 122667 & 1 2-Diphenylhydrazine & 5 \\
\hline 1815 & $99858 \mathrm{~A}$ & & Sample volume, mL, SC 1385 & 51 \\
\hline
\end{tabular}

SCHEDULE 1387

Schedule Description: Limited VOC's, using purge and trap with GC/ MS

Sample Requirements: $3 \times 40-\mathrm{mL}$ volatile organic vials (GCV), unfiltered, filled to top and capped without bubbles, put into "poly" sleeves or wrapped in bubble wrap, chilled \& maintained @ 4 deg. $\mathrm{C}$

Container Requirement: $3 \times 40-\mathrm{mL}$ VOA vials, with Teflon-lined caps, quality-control, checked and obtained from NWQL

\begin{tabular}{lcrlr}
$\begin{array}{l}\text { Lab. } \\
\text { code }\end{array}$ & $\begin{array}{c}\text { Parameter } \\
\text { code }\end{array}$ & $\begin{array}{c}\text { CAS } \\
\text { number }\end{array}$ & $\begin{array}{r}\text { MRL } \\
\text { Compound name }\end{array}$ \\
\hline & & & & \\
1287 & $34030 \mathrm{~B}$ & 71432 & Benzene & 0.2 \\
1288 & $32104 \mathrm{~B}$ & 75252 & Bromoform & .2 \\
1291 & $32105 \mathrm{~B}$ & 124481 & Chlorodibromomethane & .2 \\
1294 & $32106 \mathrm{~B}$ & 67663 & Chloroform & .2 \\
1295 & $32101 \mathrm{~B}$ & 75274 & Dichlorobromomethane & .2 \\
1303 & $34371 \mathrm{~B}$ & 100414 & Ethylbenzene & .2 \\
1305 & $34423 \mathrm{~B}$ & 75092 & Methylenechloride & .2 \\
1308 & $34010 \mathrm{~B}$ & 108883 & Toluene & .2 \\
1311 & $39180 \mathrm{~B}$ & 79016 & Trichloroethylene & .2 \\
1330 & $81551 \mathrm{~B}$ & 1330207 & Xylene & .2
\end{tabular}




\section{SCHEDULE 1389}

Schedule Description: Organonitrogen compounds, total recoverable, whole water, analyzed by GC/NPD

Sample Requirements: $800-1,000 \mathrm{~mL}$, GCC, unfiltered, chilled \& maintained@ 4 deg. C

Container Requirement: 1-L amber, Boston-round pesticide glass bottle, cleaned and burned from NWQL

\begin{tabular}{|c|c|c|c|}
\hline $\begin{array}{l}\text { Lab. } \\
\text { code }\end{array}$ & $\begin{array}{c}\text { Parameter } \\
\text { code }\end{array}$ & $\begin{array}{l}\text { CAS } \\
\text { number }\end{array}$ & Compound name \\
\hline 631 & 39057 A & 7287196 & Prometryne \\
\hline 717 & $39630 \mathrm{~A}$ & 1912249 & Atrazine \\
\hline 0718 & $39056 \mathrm{~A}$ & 1610180 & Prometone \\
\hline 0719 & $39055 \mathrm{~A}$ & 122349 & Simazine \\
\hline 0720 & 39054 A & 1014706 & Simetryn \\
\hline 0844 & $39024 \mathrm{~A}$ & 139402 & Propazine \\
\hline 0846 & 81757 A & 21725462 & Cyanazine \\
\hline 0848 & $82184 \mathrm{~A}$ & 834128 & Ametryn \\
\hline 1331 & $77825 \mathrm{C}$ & 15972608 & Alachlor \\
\hline 1332 & $39030 \mathrm{C}$ & 1582098 & Trifluralin \\
\hline 1333 & $82611 \mathrm{C}$ & 21087649 & Metribuzin \\
\hline 1334 & $82612 \mathrm{C}$ & 51218452 & Metolachlor \\
\hline 1462 & $30311 \mathrm{~A}$ & 5902512 & Terbacil \\
\hline 1463 & 30234 A & 314409 & Bromacil \\
\hline 1464 & $30245 \mathrm{~A}$ & 5234684 & Carboxin \\
\hline 1465 & $30255 \mathrm{~A}$ & 957517 & Diphenamide \\
\hline 1466 & $30264 \mathrm{~A}$ & 51235042 & Hexazinone \\
\hline 1467 & $30324 \mathrm{~A}$ & 1929777 & Vernolate \\
\hline 1468 & $30235 \mathrm{~A}$ & 23184669 & Butachlor \\
\hline 1469 & $30254 \mathrm{~A}$ & 1134232 & Cycloate \\
\hline 1470 & $30236 \mathrm{~A}$ & 2008415 & Butylate \\
\hline 1471 & 30295 A & 1918167 & Propachlor \\
\hline 1612 & $75981 \mathrm{~A}$ & 6190654 & De-ethylatrazine \\
\hline 1613 & $75980 \mathrm{~A}$ & 1007289 & De-isopropylatrazine \\
\hline 1805 & $99861 \mathrm{~A}$ & & Sample volume, mL, SC 1389 \\
\hline 1865 & $00000 \mathrm{~A}$ & & Set number SC 1389 \\
\hline
\end{tabular}

SCHEDULE 1390

Schedule Description: VOC's total recoverable, using purge and trap and $\mathrm{GC} / \mathrm{MS}$

Sample Requirements: $3 \times 40-\mathrm{mL}$ volatile organic vials (GCV), unfiltered, filled to top and capped without bubbles, put into "poly" sleeves or wrapped in bubble wrap, chilled \& maintained @ 4 deg. C

Container Requirement: $3 \times 40-\mathrm{mL}$ VOA vials, with Teflon-lined caps, quality-control, checked and obtained from NWQL

Lab. Parameter

code code

$101134030 \mathrm{~A}$

$101232104 \mathrm{~A}$

$101332102 \mathrm{~A}$

$101434301 \mathrm{~A}$

$101532105 \mathrm{~A}$

$101634311 \mathrm{~A}$

101734576 A

$101832106 \mathrm{~A}$

101932101 A

102034668 A

102134496 A

$102232103 \mathrm{~A}$

\section{CAS}

number Compound name

71432 Benzene

75252 Bromoform

56235 Carbontetrachloride

108907 Chlorobenzene

124481 Dibromochloromethane

75003 Chloroethane

110758 2-Chloroethylvinylether

67663 Chloroform

75274 Bromodichloromethane

75718 Dichlorodifluoromethane

75343 1,1-Dichloroethane

107062 1,2-Dichloroethane
MRL

(ng/L)
SCHEDULE 1390..Continued

$\begin{array}{lll}\text { Lab. Parameter } & \text { CAS } & \text { MRL } \\ \text { code code } & \text { number Compound name } & \text { (ug/L) }\end{array}$

102334501 A 75354 1,1-Dichloroethene 3

102434546 A 15660512 Trans 1,2-dichloroethene 3

102534541 A 78875 1,2-Dichloropropane 3

102734371 A 100414 Ethylbenzene 3

102834413 A 74839 Bromomethane 3

102934423 A 75092 Methylenechloride 3

103034516 A 79345 1,1,2,2-Tetrachloroethane 3

$\begin{array}{llll}1031 & 34475 \text { A } & 127184 & \text { Tetrachloroethene }\end{array}$

103234010 A 108883 Toluene 3

103334506 A 71556 1,1,1-Trichloroethane 3

103434511 A 79005 1,1,2-Trichloroethane 3

103539180 A 79016 Trichloroethene 3

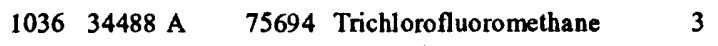

$\begin{array}{lll}1037 & 39175 \text { A } & 75014 \\ 1281 & \text { Vinylchloride } & 1\end{array}$

128134418 A 74873 Chloromethane 3

$131977651 \mathrm{C} \quad 106934$ 1,2-Dibromoethane 3

$132034536 \mathrm{C} \quad 95501$ 1,2-Dichlorobenzene 3

$132134566 \mathrm{C} \quad 541731$ 1,3-Dichlorobenzene 3

$132234571 \mathrm{C} \quad 106467$ 1,4-Dichlorobenzene 3

132334704 A 10061015 Cis-1,3-dichloropropene 3

132434699 A 10061026 Trans 1,3-dichloropropene 3

132577128 A 100425 Styrene 3

132981551 A 1330207 Xylenes (O,M,P) 3

134982625 A 96128 1,2-Dibromo-3-chloropropane 3

148581555 B 108861 Bromobenzene 3

$\begin{array}{llll}165034210 \text { A } 107028 \text { Acrolein } & 20\end{array}$

$\begin{array}{llll}1651 & 34215 \text { A } & 107131 \text { Acrylonitrile } & 20\end{array}$

165378032 B 1634044 Methyltertbutylether 5

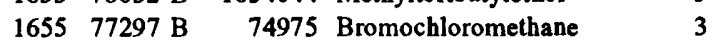

165777093 B 156592 Cis-1,2-dichloroethene 3

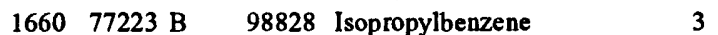

$\begin{array}{lllll}1662 & 77224 & \text { B } & 103651 & \text { n-Propylbenzene }\end{array}$

166477353 B 98066 Tert-butylbenzene 3

166677222 B 9563612 1,2,4-Trimethylbenzene 3

$\begin{array}{lllll}1668 & 77350 \text { B } & 135988 & \text { Sec-butylbenzene } & 3\end{array}$

167077356 B 99876 p-Isopropyltoluene 3

167277342 B 104518 n-Butylbenzene 3

167434551 B 12082112 1,2,4-Trichlorobenzene 3

167639702 B 87683 Hexachlorobutadiene 3

$\begin{array}{llll}1678 & 34696 \text { B } & 91203 & \text { Naphthalene }\end{array}$

$\begin{array}{llllll}1680 & 77613 & \text { B } & 8761612 & 1,2,3-T r i c h l o r o b e n z e n e & 3\end{array}$

168277652 B 76131 Trichlorotrifluoroethane 3

$\begin{array}{lllll}1684 & 77226 & B & 108678 & 1,3,5-T r i m e t h y l b e n z e n e\end{array} 3$

$\begin{array}{lllll}1689 & 77168 \text { A } 563586 & \text { 1,1-Dichloropropene } 3\end{array}$

169030217 A 74953 Dibromomethane 3

$\begin{array}{llll}1691 & 77170 \text { A } 59420722 & \text { 2,2-Dichloropropane } & 3\end{array}$

169277173 A 14228913 1,3-Dichloropropane 3

169377562 A 630206 1,1,1,2-Tetrachloroethane 3

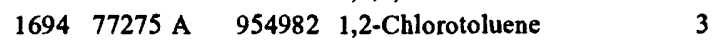

169577443 A 96184123 1,2,3-Trichloropropane 3

169677277 A 106434 1,4-Chlorotoluene 3 


\section{SCHEDULE 1392}

Schedule Description: VOC, total recoverable, using purge and trap and GC/MS with unknowns tentatively identified

Sample Requirements: $3 \times 40-\mathrm{mL}$ volatile organic vials (GCV), unfiltered filled to top and capped without bubbles, put into "poly" sleeves or wrapped in bubble wrap, chilled \& maintained @ 4 deg. C

Container Requirement: 3x40-mL VOA vials, with Teflon-lined caps, quality-control, checked and obtained from NWQL

\begin{tabular}{|c|c|c|c|c|}
\hline $\begin{array}{l}\text { Lab. } \\
\text { code }\end{array}$ & $\begin{array}{c}\text { Parameter } \\
\text { code }\end{array}$ & $\begin{array}{l}\text { CAS } \\
\text { number }\end{array}$ & Compound name & $\begin{array}{l}\text { MRL } \\
(1 \mathrm{~g} / \mathrm{L})\end{array}$ \\
\hline 1009 & 30217 B & 74953 & Dibromomethane & 0 \\
\hline 1287 & $34030 \mathrm{~B}$ & 71432 & Benzene & 2 \\
\hline 1288 & $32104 \mathrm{~B}$ & 75252 & Bromoform & 2 \\
\hline 1289 & $32102 \mathrm{~B}$ & 56235 & Carbontetrachloride & 2 \\
\hline 1290 & $34301 \mathrm{~B}$ & 108907 & Chlorobenzene & 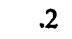 \\
\hline 1291 & $32105 \mathrm{~B}$ & 124481 & Dibromochloromethane & 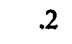 \\
\hline 1292 & $34311 \mathrm{~B}$ & 75003 & Chloroethane & 2 \\
\hline 1294 & $32106 \mathrm{~B}$ & 67663 & Chloroform & .2 \\
\hline 1295 & $32101 \mathrm{~B}$ & 75274 & Bromodichloromethane & .2 \\
\hline 1296 & $34668 \mathrm{~B}$ & 75718 & Dichlorodifluoromethane & .2 \\
\hline 1297 & 34496 B & 75343 & 1,1-Dichloroethane & 2 \\
\hline 1298 & $32103 \mathrm{~B}$ & 107062 & 1,2-Dichloroethane & .2 \\
\hline 1299 & $34501 \mathrm{~B}$ & 75354 & 1,1-Dichlorethene & 2 \\
\hline 1300 & $34546 \mathrm{~B}$ & 15660512 & Trans 1,2-dichloroethene & .2 \\
\hline 1301 & $34541 \mathrm{~B}$ & 78875 & 1,2-Dichloropropane & .2 \\
\hline 1303 & $34371 \mathrm{~B}$ & 100414 & Ethylbenzene & .2 \\
\hline 1304 & 34413 B & 74839 & Bromomethane & .2 \\
\hline 1305 & 34423 B & 75092 & Methylenechloride & .2 \\
\hline 1306 & $34516 \mathrm{~B}$ & 79345 & 1,1,2,2-Tetrchloroethane & .2 \\
\hline 1307 & 34475 B & 127184 & Tetrachloroethene & .2 \\
\hline 1308 & $34010 \mathrm{~B}$ & 108883 & Toluene & .2 \\
\hline 1309 & $34506 \mathrm{~B}$ & 71556 & 1,1,1-Trichloroethane & .2 \\
\hline 1310 & $34511 \mathrm{~B}$ & 79005 & 1,1,2-Trichloroethane & .2 \\
\hline 1311 & $39180 \mathrm{~B}$ & 79016 & Trichloroethene & .2 \\
\hline 1312 & 34488 B & 75694 & Trichlorofluoromethane & .2 \\
\hline 1313 & $39175 \mathrm{~B}$ & 75014 & Vinylchloride & .2 \\
\hline 1315 & $34566 \mathrm{~B}$ & 541731 & 1,3-Dichlorobenzene & .2 \\
\hline 1316 & $34571 \mathrm{~B}$ & 106467 & 1,4-Dichlorobenzene & .2 \\
\hline 1317 & $77651 \mathrm{~B}$ & 106934 & 1,2-Dibromoethane & .2 \\
\hline 1318 & 34418 B & 74873 & Chloromethane & .2 \\
\hline 1320 & $34536 \mathrm{C}$ & 95501 & 1,2-Dichlorobenzene & .2 \\
\hline 1326 & $34704 \mathrm{~B}$ & 10061015 & Cis-1,3-Dichloropropene & .2 \\
\hline 1327 & 34699 B & 10061026 & Trans-1,3-Dichloropropene & .2 \\
\hline 1328 & $77128 \mathrm{~B}$ & 100425 & Styrene & .2 \\
\hline 1330 & $81551 \mathrm{~B}$ & 1330207 & Xylenes $(\mathrm{O}, \mathrm{M}, \mathrm{P})$ & .2 \\
\hline 1354 & $82625 \mathrm{~B}$ & 96128 & 1,2-Dibromo-3-chloropropane & 1 \\
\hline 1478 & $77168 \mathrm{~B}$ & 56358611 & 1,1-Dichloropropene & .2 \\
\hline 1479 & $77170 \mathrm{~B}$ & 59420722 & 2,2-Dichloropropane & .2 \\
\hline 1480 & $77173 \mathrm{~B}$ & 14228913 & 1,3-Dichloropropane & .2 \\
\hline 1481 & $77275 \mathrm{~B}$ & 9549812 & 2-Chlorotoluene & .2 \\
\hline 1482 & $77277 \mathrm{~B}$ & 10643414 & 4Chlorotoluene & .2 \\
\hline 1483 & $77443 \mathrm{~B}$ & 96184123 & 1,2,3-Trichloropropane & .2 \\
\hline 1484 & $77562 \mathrm{~B}$ & 630206 & 1,1,1,2-Tetrachloroethane & .2 \\
\hline 1650 & 34210 A & 107028 & Acrolein & 20 \\
\hline 1651 & 34215 A & 107131 & Acrylonitrile & 20 \\
\hline 1652 & $78032 \mathrm{~A}$ & 1634044 & Methyltertbutylether & 1 \\
\hline 1654 & 77297 A & 74975 & Bromochloromethane & .2 \\
\hline 1656 & 77093 A & 156592 & Cis-1,2-dichloroethene & .2 \\
\hline 1658 & $34576 \mathrm{C}$ & 1107582 & Chloroethylvinylether & 1 \\
\hline 1659 & 77223 A & 98828 & Isopropylbenzene & .2 \\
\hline 1661 & $77224 \mathrm{~A}$ & 103651 & n-Propylbenzene & .2 \\
\hline
\end{tabular}

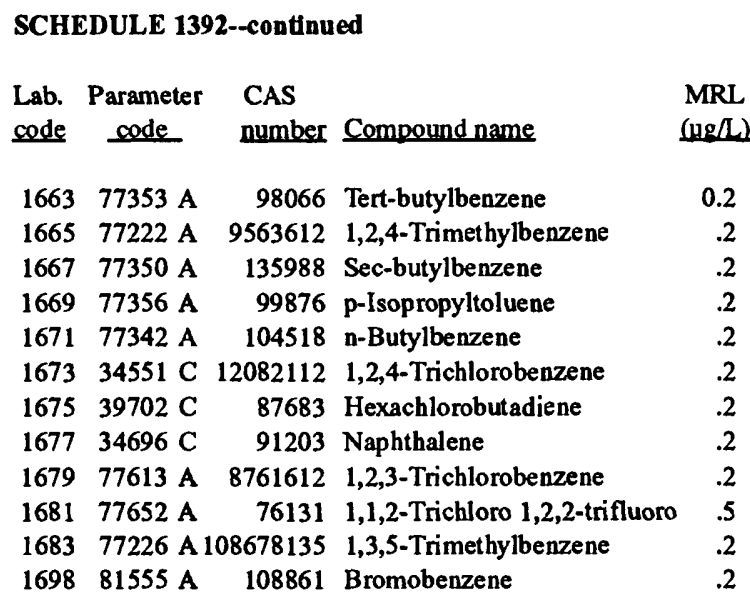

\section{SCHEDULE 1393}

Schedule Description: Acid organic compounds, total recoverable from whole water, using methylene chloride and GCMS

Sample Requirements: 800-1,000 mL, GCC, unfiltered, chilled \& maintained @4 deg. C

Container Requirement: 1-L amber, Boston-round pesticide glass bottle, cleaned and burned from NWQL

$\begin{array}{lcclr}\begin{array}{l}\text { Lab. } \\ \text { code }\end{array} & \begin{array}{c}\text { Parameter } \\ \text { code }\end{array} & \begin{array}{c}\text { CAS } \\ \text { number }\end{array} & \begin{array}{r}\text { Compound name } \\ \text { (ug/L) }\end{array} \\ & & & & \\ 1055 & 34452 \text { A } & 59507 & \text { Chloro-methylphenol } & 30 \\ 1056 & 34586 \text { A } & 95578 & \text { 2-Chlorophenol } & 5 \\ 1057 & 34601 \text { A } & 120832 & \text { 2,4-Dichlorophenol } & 5 \\ 1058 & 34621 \text { A } & 88062 & \text { 2,4,6-Trichlorophenol } & 20 \\ 1059 & 34606 \text { A } & 105679 & \text { 2,4-Dimethylphenol } & 5 \\ 1060 & 34657 \text { A } & 534521 & \text { 1,6-Dinitro-2-methylphenol } & 30 \\ 1061 & 34616 \text { A } & 51285 & \text { 2,4-Dinitrophenol } & 20 \\ 1062 & 34591 \text { A } & 88755 & \text { 2-Nitrophenol } & 5 \\ 1063 & 34646 \text { A } & 100027 & \text { 4Nitrophenol } & 30 \\ 1064 & 39032 \text { A } & 87865 & \text { Pentachlorophenol } & 30 \\ 1065 & 34694 \text { A } & 108952 & \text { Phenol } & 5\end{array}$




\section{SCHEDULE 1394}

Schedule Description: Base/neutral organic compounds, total recoverable from whole water, using methylene chloride and GC/MS

Sample Requirements: 800-1,000 mL, GCC, unfiltered, chilled \& maintained @ 4 deg. C

Container Requirement: 1-L amber, Boston-round pesticide glass bottle, cleaned and burned from NWQL

Lab. Parameter

code code

CAS

number Compound name

MRL

$106634205 \mathrm{~A}$

83329 Acenaphthene

$106734200 \mathrm{~A}$

$106834220 \mathrm{~A}$

$107034526 \mathrm{~A}$

$107134230 \mathrm{~A}$

$107234242 \mathrm{~A}$

107334247 A

$107434521 \mathrm{~A}$

$107534292 \mathrm{~A}$

$107634278 \mathrm{~A}$

$107734273 \mathrm{~A}$

$107834283 \mathrm{~A}$

107934636 A

$108034581 \mathrm{~A}$

$108134641 \mathrm{~B}$

$108234320 \mathrm{~A}$

108334556 A

$108439110 \mathrm{~A}$

$108534536 \mathrm{~A}$

$108634566 \mathrm{~A}$

108734571 A

108934336 A

109034341 A

$109134611 \mathrm{~A}$

109234626 A

109334596 A

$109439100 \mathrm{~A}$

$109534381 \mathrm{~A}$

109634376 A

$109739700 \mathrm{~A}$

$109834391 \mathrm{~A}$

109934386 A

110034396 A

$110134403 \mathrm{~A}$

110234408 A

$110334696 \mathrm{~A}$

110434447 A

$110534438 \mathrm{~A}$

110634433 A

110734428 A

110834461 A

110934469 A

111134551 A
208968 Acenaphthylene

120127 Anthracene

56553 Benzo[a]anthracene

205992 Benzo[b]fluoranthene

207089 Benzo[k]fluoranthene

50328 Benzo[a]pyrene

191242 Benzo[g,h,i] perylene

85687 Butyl benzyl phthalate

111911 Bis (2-chloroethoxy) methane

111444 Bis (2-chlorethy) ether 5

108601 Bis (2-chlorisopropyl) ether

101553 4-Bromophenylphenyether

91587 2-Chloronaphthalene

7005723 4-Chlorophenylphenyether

218019 Chrysene

53703 1,2,5,6-Dibenz[a,h]anthracene 10

84742 Di-n-butyl phthalate 5

95501 1,2-Dichlorobenzene 5

541731 1,3-Dichlorobenzene 5

106467 1,4-Dichlorobenzene 5

84662 Diethyl phthalate 5

131113 Dimethyl phthalate 5

121142 2,4-Dinitrotoluene 5

606202 2,6-Dinitrotoluene 5

117817 Bis (2-ethlyhexyl) phthalate

86737 Fluorene

206440 Fluoranthene

118741 Hexachlorobenzene

87683 Hexachlorobutadiene

77474 Hexachlorocyclopentadiene

67721 Hexachloroethane

193395 Indeno(1,2,3-cd) pyrene 10

78591 Isophorone

91203 Naphthalene

98953 Nitrobenzene

62759 n-Nitrosodimethlyamine

86306 n-Nitrosodiphenylamine

621647 n-Nitrosodi-n-propylamine

85018 Phenanthrene

129000 Pyrene

120821 1,2,4-Trichlorobenzene
117840 Di-n-octyl phthalate 10
SCHEDULE 1398

Schedule Description: Organochloride pesticides with PCB's and PCN's, total recoverable from whole water

Sample Requirements: 800-1,000 mL, GCC, unfiltered, chilled \& maintained @ 4 deg. C

Container Requirement: 1-L amber, Boston-round pesticide glass bottle, cleaned and burned from NWQL

\begin{tabular}{|c|c|c|c|c|}
\hline $\begin{array}{l}\text { Lab. } \\
\text { code }\end{array}$ & $\begin{array}{c}\text { Parameter } \\
\text { code }\end{array}$ & $\begin{array}{l}\text { CAS } \\
\text { number }\end{array}$ & Compound name & $\begin{array}{l}\text { MRL } \\
(\text { ng/M) }\end{array}$ \\
\hline 348 & $39034 \mathrm{~A}$ & 72560 & Perthane & 0.1 \\
\hline 0351 & $39350 \mathrm{~B}$ & 57749 & Chlordane & .1 \\
\hline 0360 & $39400 \mathrm{~B}$ & 8001352 & Toxaphene & 1 \\
\hline 0392 & 39516 B & 12767792 & PCB's, gross & .1 \\
\hline 0393 & $39250 \mathrm{~B}$ & 25104556 & PCN's, gross & .1 \\
\hline 0400 & $39480 \mathrm{~B}$ & 72435 & Methoxychlor & .01 \\
\hline 0544 & 39755 B & 2385855 & Mirex & .01 \\
\hline 0737 & $39388 \mathrm{~B}$ & 115297 & Endosulfan I & .001 \\
\hline 0738 & $39330 \mathrm{~B}$ & 309002 & Aldrin & .001 \\
\hline 0739 & 39360 B & 72548 & DDD & .001 \\
\hline 0740 & 39365 B & 72559 & DDE & .001 \\
\hline 0741 & $39370 \mathrm{~B}$ & 50293 & DDT & .001 \\
\hline 0742 & $39380 \mathrm{~B}$ & 60571 & Dieldrin & .001 \\
\hline 0743 & $39390 \mathrm{~B}$ & 72208 & Endrin & .001 \\
\hline 0744 & $39410 \mathrm{~B}$ & 76448 & Heptachlor & .001 \\
\hline 0745 & $39420 \mathrm{~B}$ & 1024573 & Haptachlor epoxide & .001 \\
\hline 0746 & 39340 B & 58899 & Lindane & .001 \\
\hline 1807 & 99864 A & & Sample volume, mL, SC 1398 & 1 \\
\hline 1869 & $00000 \mathrm{~A}$ & & Set number SC 1398 & \\
\hline
\end{tabular}

\section{SCHEDULE 1399}

Schedule Description: Organochlorine and organophosphate pesticides with PCB's and PCN's, total recoverable from whole water

Sample Requirements: 800-1,000 mL, GCC, unfiltered, chilled \& maintained @ 4 deg. C

Container Requirement: 1-L amber, Boston-round pesticide glass bottle, cleaned and burned from NWQL

\begin{tabular}{|c|c|c|c|c|}
\hline $\begin{array}{l}\text { Lab. } \\
\text { code }\end{array}$ & $\begin{array}{c}\text { Parameter } \\
\text { code }\end{array}$ & $\begin{array}{l}\text { CAS } \\
\text { number }\end{array}$ & Compound name & $\begin{array}{c}\text { MRL } \\
(1 \mathrm{~g} / \mathrm{L})\end{array}$ \\
\hline 0348 & $39034 \mathrm{~A}$ & 72560 & Perthane & 0.1 \\
\hline 0351 & $39350 \mathrm{~B}$ & 57749 & Chlordane & .1 \\
\hline 0360 & $39400 \mathrm{~B}$ & 8001352 & Toxaphene & 1 \\
\hline 0378 & $39570 \mathrm{~B}$ & 333415 & Diazinon & .01 \\
\hline 0379 & 39398 B & 563122 & Ethion & .01 \\
\hline 0380 & $39530 \mathrm{~B}$ & 121755 & Malathion & .01 \\
\hline 0381 & $39600 \mathrm{~B}$ & 298000 & Methylparathion & .01 \\
\hline 0383 & $39540 \mathrm{~B}$ & 56382 & Parathion & .01 \\
\hline 0384 & $39786 \mathrm{~B}$ & 786196 & Trithion & .01 \\
\hline 0392 & $39516 \mathrm{~B}$ & 12767792 & PCB's, gross & .1 \\
\hline 0393 & $39250 \mathrm{~B}$ & 25104556 & PCN's, gross & .1 \\
\hline 0400 & $39480 \mathrm{~B}$ & 72435 & Methoxychlor & .01 \\
\hline 0544 & 39755 B & 2385855 & Mirex & .01 \\
\hline 0737 & $39388 \mathrm{~B}$ & 115297 & Endosulfan I & .001 \\
\hline 0738 & $39330 \mathrm{~B}$ & 309002 & Aldrin & .001 \\
\hline 0739 & $39360 \mathrm{~B}$ & 72548 & DDD & .001 \\
\hline 0740 & $39365 \mathrm{~B}$ & 72559 & DDE & .001 \\
\hline 0741 & $39370 \mathrm{~B}$ & 50293 & DDT & .001 \\
\hline 0742 & $39380 \mathrm{~B}$ & 60571 & Dieldrin & .001 \\
\hline 0743 & $39390 \mathrm{~B}$ & 72208 & Endrin & .001 \\
\hline 0744 & $39410 \mathrm{~B}$ & 76448 & Heptachlor & .001 \\
\hline 0745 & $39420 \mathrm{~B}$ & 1024573 & Heptachlor epoxide & .001 \\
\hline 0746 & 340 & 58899 & Lindane & .001 \\
\hline
\end{tabular}




\section{SCHEDULE 1401}

Schedule Description: Acrolein, acrylonitrile, and 2-chloroethylvinylether total recoverable, from whole water using purge and trap and GC/MS

Sample Requirements: $3 \times 40-\mathrm{mL}$ volatile organic vials (GCV), unfiltered filled to top and capped without bubbles, put into "poly" sleeves or wrapped in bubble wrap, chilled \& maintained @ 4 deg. $\mathrm{C} ; \mathrm{pH}$ adjusted between 4 to 5 with 1:4 $\mathrm{HCl}$ :water

Container Requirement: $3 \times 40-\mathrm{mL}$ VOA vials, with Teflon-lined caps, quality-control, checked and obtained from NWQL

$\begin{array}{lcclc}\begin{array}{l}\text { Lab. } \\ \text { code }\end{array} & \begin{array}{c}\text { Parameter } \\ \text { code }\end{array} & \begin{array}{c}\text { CAS } \\ \text { number }\end{array} & \begin{array}{c}\text { Compound name } \\ \text { (ng/L) }\end{array} \\ & & & \\ 1650 & 34210 \mathrm{~A} & 107028 & \text { Acrolein } & 20 \\ 1651 & 34215 \mathrm{~A} & 107131 & \text { Acrylonitrile } & 20 \\ 1658 & 34576 \mathrm{C} & 1107582 & \text { 2-Chloroethylvinylether } & 1\end{array}$

\section{SCHEDULE 1474}

Schedule Description: Onganochlorine and organophosphate pesticides with PCB's and PCN's, total recoverable from whole water, analyzed by $G C / E C D$

Sample Requirements: 1,650-2,000 mL, GCC, collected unfiltered chilled \& maintained @ 4 deg. C

Container Requirement: 2-L amber, Boston-round pesticide glass bottle, cleaned and burned from NWQL

\begin{tabular}{|c|c|c|c|}
\hline $\begin{array}{l}\text { Lab. } \\
\text { code }\end{array}$ & $\begin{array}{c}\text { Parameter } \\
\text { code }\end{array}$ & $\begin{array}{l}\text { CAS } \\
\text { number }\end{array}$ & Compound name \\
\hline 348 & $39034 \mathrm{~A}$ & 72560 & Perthane \\
\hline 0349 & $39388 \mathrm{C}$ & 115297 & Endosulfan I \\
\hline 0350 & $39330 \mathrm{C}$ & 309002 & Aldrin \\
\hline 0351 & $39350 \mathrm{~B}$ & 57749 & Chlordane \\
\hline 0352 & $39360 \mathrm{C}$ & 72548 & DDD \\
\hline 0353 & $39365 \mathrm{C}$ & 72559 & DDE \\
\hline 0354 & $39370 \mathrm{C}$ & 50293 & DDT \\
\hline 0355 & $39380 \mathrm{C}$ & 60571 & Dieldrin \\
\hline 0356 & $39390 \mathrm{C}$ & 72208 & Endrin \\
\hline 0357 & $39410 \mathrm{C}$ & 76448 & Heptachlor \\
\hline 0358 & $39420 \mathrm{C}$ & 1024573 & Heptachlor epoxide \\
\hline 0359 & $39340 \mathrm{C}$ & 58899 & Lindane \\
\hline 0360 & $39400 \mathrm{~B}$ & 8001352 & Toxaphene \\
\hline 0372 & $39730 \mathrm{~B}$ & 94757 & $2,4-D$ \\
\hline 0373 & $39740 \mathrm{~B}$ & 93765 & $2,4,5-T$ \\
\hline 0374 & $39760 \mathrm{~B}$ & 93721 & Silvex \\
\hline 0378 & $39570 \mathrm{~B}$ & 333415 & Diazinon \\
\hline 0379 & $39398 \mathrm{~B}$ & 563122 & Ethion \\
\hline 0380 & $39530 \mathrm{~B}$ & 121755 & Malathion \\
\hline 0381 & $39600 \mathrm{~B}$ & 298000 & Methylparathion \\
\hline 0383 & $39540 \mathrm{~B}$ & 56382 & Parathion \\
\hline 0384 & 39786 B & 786196 & Trithion \\
\hline 0392 & $39516 \mathrm{~B}$ & 12767792 & Gross PCB \\
\hline 0393 & $39250 \mathrm{~B}$ & 25104556 & Gross PCN \\
\hline 0400 & $39480 \mathrm{~B}$ & 72435 & Methoxychlor \\
\hline 0402 & $82183 \mathrm{~A}$ & 120365 & 2,4-DP \\
\hline 0544 & 39755 B & 2385855 & Mirex \\
\hline 0592 & $39011 \mathrm{~A}$ & 298044 & Di-Syston (disulfoton) \\
\hline 0593 & $39023 \mathrm{~A}$ & 298022 & Phorate \\
\hline 0753 & 38932 A & 2921882 & Chlorpyrifos \\
\hline 0802 & $39040 \mathrm{~A}$ & 78488 & DEF \\
\hline 13 & 82614 & 944229 & Fonofos \\
\hline
\end{tabular}

$\begin{array}{ccc}\text { MRL } & 1642 & 39496 \mathrm{D} \\ \text { (1)/L) } & 1643 & 39500 \mathrm{D} \\ & 1644 & 39504 \mathrm{D} \\ 0.1 & 1645 & 39508 \mathrm{D}\end{array}$

.01

.01

.1

.01

.01

.01

.01

.01

.01

.01

.01

1

.01

.01

.01

.01

.01

.01

.01

.01

.01

.1

.1

.01

.01

.01

.01

.01

.01

.01

.01

\section{SCHEDULE 1608}

Schedule Description: Organochlorine pesticides with individual Aroclor PCB's (USEPA-608), total recoverable from whole water

Sample Requirements: 800-1,000 mL, GCC, unfiltered, chilled \& maintained@4 deg.C

Container Requirement: 1-L amber, Boston-round pesticide glass bottle, cleaned and burned from NWQL

\begin{tabular}{|c|c|c|c|c|}
\hline $\begin{array}{l}\text { Lab. } \\
\text { code }\end{array}$ & $\begin{array}{c}\text { Parameter } \\
\text { code }\end{array}$ & $\begin{array}{l}\text { CAS } \\
\text { number }\end{array}$ & Compound name & $\begin{array}{l}\text { MRL } \\
(\lg / \text { L) }\end{array}$ \\
\hline 1619 & $39337 \mathrm{D}$ & 319846 & Alpha-BHC & 0.03 \\
\hline 1620 & $39338 \mathrm{D}$ & 319857 & Beta-BHC & .03 \\
\hline 1621 & $39340 \mathrm{D}$ & 58899 & Gamma, BHC (lindane) & .03 \\
\hline 1622 & $34259 \mathrm{D}$ & 319868 & Delta-BHC & .09 \\
\hline 1623 & $39410 \mathrm{D}$ & 76448 & Heptachlor & .03 \\
\hline 1624 & $39330 \mathrm{D}$ & 309002 & Aldrin & .04 \\
\hline 1625 & $39420 \mathrm{D}$ & 1024573 & Heptachlor expoxide & .8 \\
\hline 1626 & $39065 \mathrm{D}$ & 5103742 & Chlordane-trans & .1 \\
\hline 1627 & $34361 \mathrm{D}$ & 959988 & Endosulfan I (alpha) & .1 \\
\hline 1628 & $39062 \mathrm{D}$ & 5103719 & Chlordane-cis & .1 \\
\hline 1629 & $39380 \mathrm{D}$ & 60571 & Dieldrin & .02 \\
\hline 1630 & $39320 \mathrm{D}$ & 725594 & 4,4' DDE & .04 \\
\hline 1641 & $34671 \mathrm{D}$ & & Aroclor 1016 & .1 \\
\hline 1639 & $39488 \mathrm{D}$ & & Aroclor 1221 & .1 \\
\hline 1640 & $39492 \mathrm{D}$ & & Aroclor 1232 & .1 \\
\hline 1642 & $39496 \mathrm{D}$ & & Aroclor 1242 & .1 \\
\hline 1643 & $39500 \mathrm{D}$ & & Aroclor 1248 & .1 \\
\hline 1644 & $39504 \mathrm{D}$ & & Aroclor 1254 & .1 \\
\hline 1645 & $39508 \mathrm{D}$ & & Aroclor 1260 & .1 \\
\hline
\end{tabular}


SCHEDULE 2001

Schedule Description: Pesticides in filtered water extracted by NWQL on C-18 SPE cartridge and analyzed by GC/MS

Sample Requirements: $1 \mathrm{~L}$ of water filtered through $0.7-\mu \mathrm{m}$ glass fiber depth filter, chilled at $4 \mathrm{deg}$. $\mathrm{C}$ (packed in ice)

Container Requirements: 1-L baked amber glass bottle, GCC, from NWQL

\begin{tabular}{|c|c|c|c|c|}
\hline $\begin{array}{l}\text { Lab. } \\
\text { code }\end{array}$ & $\begin{array}{c}\text { Parameter } \\
\text { code }\end{array}$ & $\begin{array}{l}\text { CAS } \\
\text { number }\end{array}$ & Compound name & $\begin{array}{l}\text { MRL } \\
(\operatorname{lng} / \mathrm{L})\end{array}$ \\
\hline & $46342 \mathrm{D}$ & 15972608 & Alachlor & 0.009 \\
\hline 002 & $04040 \mathrm{D}$ & 6190654 & Atrazine,Desethyl- & .007 \\
\hline 03 & $39632 \mathrm{D}$ & 1912249 & Atrazine & .017 \\
\hline 004 & 82686 D & 86500 & Azinphos,Methyl- & .038 \\
\hline 4005 & 82673 D & 1861401 & Benfluralin & .013 \\
\hline 4006 & $04028 \mathrm{D}$ & 2008415 & Butylate & .008 \\
\hline 4007 & 82680 D & 63252 & Carbaryl(Sevin) & .046 \\
\hline 4008 & 82674 D & 1563662 & Carbofuran & .013 \\
\hline 4009 & $38933 \mathrm{D}$ & 2921882 & Chlorpyrifos & .005 \\
\hline 4010 & $04041 \mathrm{D}$ & 21725462 & Cyanazine & .013 \\
\hline 4011 & $82682 \mathrm{D}$ & 1861321 & DCPA(Dacthal) & .004 \\
\hline 4012 & $34653 \mathrm{D}$ & 72559 & DDE,p,p'- & .010 \\
\hline 4013 & $39572 \mathrm{D}$ & 333415 & Diazinon & .008 \\
\hline 4015 & $39381 \mathrm{D}$ & 60571 & Dieldrin & .008 \\
\hline 4016 & $82660 \mathrm{D}$ & 579668 & Diethylaniline & .006 \\
\hline 4017 & 82662 D & 60515 & Dimethoate & .024 \\
\hline 4018 & $82677 \mathrm{D}$ & 298044 & Disulfoton & .028 \\
\hline 4019 & $82668 \mathrm{D}$ & 759944 & EPTC(Eptam) & .005 \\
\hline 4020 & 82663 D & 55283686 & Ethalfluralin & .013 \\
\hline 4021 & $82672 \mathrm{D}$ & 13194484 & Ethoprop & .012 \\
\hline 4022 & $04095 \mathrm{D}$ & 944229 & Fonofos & .008 \\
\hline 4023 & $34253 \mathrm{D}$ & 319846 & HCH, alpha- & .007 \\
\hline 4025 & $39341 \mathrm{D}$ & 58899 & $\mathrm{HCH}$, gamma-(Lindane) & .011 \\
\hline 4026 & 82666 D & 330552 & Linuron & .039 \\
\hline 4027 & $39532 \mathrm{D}$ & 21755 & Malathion & .010 \\
\hline 4029 & $39415 \mathrm{D}$ & 51218452 & Metolachlor & .009 \\
\hline 4030 & $82630 \mathrm{D}$ & 21087649 & Metribuzin & .012 \\
\hline 4031 & $82671 \mathrm{D}$ & 2212671 & Molinate & .007 \\
\hline 4032 & $82684 \mathrm{D}$ & 15299997 & Napropamide & .010 \\
\hline 4033 & $39542 \mathrm{D}$ & 56382 & Parathion,Ethyl- & .022 \\
\hline 4028 & $82667 \mathrm{D}$ & 298000 & Parathion,Methyl- & .035 \\
\hline 4034 & $82669 \mathrm{D}$ & 1114712 & Pebulate & .009 \\
\hline 4035 & $82683 \mathrm{D}$ & 40487421 & Pendimethalin & .018 \\
\hline 4036 & $82687 \mathrm{D}$ & 52645531 & Permethrin,cis- & .019 \\
\hline 4037 & $82664 \mathrm{D}$ & 298022 & Phorate & .011 \\
\hline 4038 & $82676 \mathrm{D}$ & 23950585 & Pronamide & .009 \\
\hline 4039 & $04037 \mathrm{D}$ & 1610180 & Prometon & .008 \\
\hline 4040 & $04024 \mathrm{D}$ & 1918167 & Propachlor & .015 \\
\hline 4041 & 82679 D & 709988 & Propanil & .016 \\
\hline 4042 & 82685 D & 2312358 & Propargite & .006 \\
\hline 4043 & $04035 \mathrm{D}$ & 122349 & Simazine & .008 \\
\hline 4044 & $82681 \mathrm{D}$ & 28249776 & Thiobencarb & .008 \\
\hline 4045 & 82670 D & 34014181 & Tebuthiuron & .015 \\
\hline 4046 & $82665 \mathrm{D}$ & 5902512 & Terbacil & .030 \\
\hline 4047 & 82675 D & 13071799 & Terbufos & .012 \\
\hline 4049 & 82678 D & 2303175 & Triallate & .008 \\
\hline 4050 & 82661 D & 1582098 & Trifluralin & .012 \\
\hline 4014 & $91063 \mathrm{D}$ & & Diazinon, d10-surrogate \% & \\
\hline 4024 & $91065 \mathrm{D}$ & & $\mathrm{HCH}$, alpha, d6-surrogate $\%$ & \\
\hline 4048 & $91064 \mathrm{D}$ & 5915413 & Terbuthylazine surrogate \% & \\
\hline 4051 & $99856 \mathrm{D}$ & & Sample volume in $\mathrm{mL}$ & \\
\hline 4052 & $00000 \mathrm{~A}$ & & Set number SC 2001 & \\
\hline
\end{tabular}

SCHEDULE 2010

Schedule Description: Pesticides in filtered water field extracted on C-18 SPE cartridge and analyzed by GC/MS

Sample Requirements: 1 C-18 SPE cartridge through which approximately $1 \mathrm{~L}$ of water filtered through a $0.7-\mu \mathrm{m}$ glass fiber depth filter was processed, annotate actual volume on cartridge form, chilled@ 4 deg. C (packed in ice)

Container Requirements: $40-\mathrm{mL}$ amber glass septum cap vial

Lab. Parameter CAS MRI

code code number Compound name (ug/L)

420146342 E 15972608 Alachlor 0.009

420204040 E 6190654 Atrazine,Desethyl- 007

$420339632 \mathrm{E} 1912249$ Atrazine 017

$\begin{array}{llll}4204 & 82686 \mathrm{E} \quad 86500 & \text { Azinphos,Methyl- } & .038\end{array}$

$420582673 \mathrm{E} 1861401$ Benfluralin

$420604028 \mathrm{E} 2008415$ Butylate 008

$\begin{array}{llll}4207 & 82680 \mathrm{E} & 63252 & \text { Carbaryl(Sevin) }\end{array}$

420882674 E 1563662 Carbofuran

$420938933 \mathrm{E} 2921882$ Chlorpyrifos

$421004041 \mathrm{E} 21725462$ Cyanazine 013

$421182682 \mathrm{E} \quad 1861321$ DCPA(Dathal)

421234653 E 72559 DDE,p,p'-

$\begin{array}{llll}4213 & 39572 \mathrm{E} & 333415 & \text { Diazinon }\end{array}$

$421539381 \mathrm{E} \quad 60571$ Dieldrin $\quad .008$

$\begin{array}{llrl}4216 & 82660 \mathrm{E} \quad 579668 & \text { Diethylaniline } & .006\end{array}$

$\begin{array}{llll}4217 & 82662 \mathrm{E} & 60515 & \text { Dimethoate }\end{array}$

$\begin{array}{llll}4218 & 82677 \mathrm{E} & 298044 & \text { Disulfoton }\end{array}$

421982668 E 759944 EPTC(Eptam)

422082663 E 55283686 Ethalfluralin .013

422182672 E 13194484 Ethoprop $\quad .012$

$\begin{array}{llll}4222 & 04095 \mathrm{E} \quad 944229 \text { Fonofos } & .008\end{array}$

422334253 E 319846 HCH,alpha- $\quad 007$

$422539341 \mathrm{E} \quad 58899 \mathrm{HCH}$,gamma-(Lindane) $\quad .011$

$\begin{array}{llll}4226 & 82666 \mathrm{E} & 330552 & \text { Linuron }\end{array}$

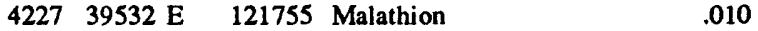

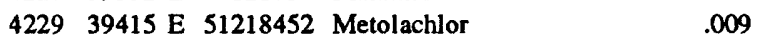

$\begin{array}{lll}4230 & 82630 \text { E } 21087649 \text { Metribuzin } & 012\end{array}$

$423182671 \mathrm{E} \quad 2212671$ Molinate

$\begin{array}{llll}4232 & 82684 \text { E } 15299997 & \text { Napropamide } & .010\end{array}$

$4233 \quad 39542$ E 56382 Parathion, Ethyl- 022

422882667 E 298000 Parathion, Methyl- 035

$423482669 \mathrm{E} 1114712$ Pebulate 009

423582683 E 40487421 Pendimethalin 018

423682687 E 52645531 Permethrin,cis- $\quad 019$

423782664 E 298022 Phorate 011

423804037 E 23950585 Pronamide .009

$423982676 \mathrm{E} 1610180$ Prometon 008

424004024 E 1918167 Propachlor $\quad .015$

$\begin{array}{llll}4241 & 82679 \mathrm{E} & 709988 & \text { Propanil }\end{array}$

$\begin{array}{lll}4242 & 82685 \text { E } 2312358 \text { Propargite } & .006\end{array}$

$\begin{array}{lllll}4243 & 04035 \text { E } 122349 \text { Simazine } & .008\end{array}$

424482670 E 28249776 Thiobencarb

424582665 E 34014181 Tebuthiuron

$\begin{array}{llll}4246 & 82675 \mathrm{E} & 5902512 & \text { Terbacil }\end{array}$

$\begin{array}{llllll}4248 & 82681 \mathrm{E} & 13071799 & \text { Terbufos } & .012\end{array}$

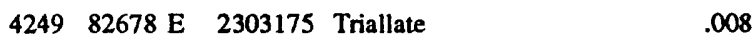

$425082661 \mathrm{E} \quad 1582098$ Trifluralin $\quad 012$

421491063 E Diazinon,d10- surrogate \%

$422491065 \mathrm{E} \quad \mathrm{HCH}, \mathrm{alpha,d6-surrogate} \%$

424791064 E 5915413 Terbuthylazine surrogate \%

$425199857 \mathrm{E} \quad$ Sample volume in $\mathrm{mL}$

4252 Set number SC 2010 


\section{SCHEDULE 2050}

Schedule Description: Pesticides in filtered water extracted by NWQL on Carbopak B SPE cartridge and analyzed by HPLC

Sample Requirements: $1 \mathrm{~L}$ of water filtered through $0.7-\mu \mathrm{m}$ glass fiber depth filter, chilled at $4 \mathrm{deg}$. $\mathrm{C}$ (packed in ice)

Container Requirements: 1- $L$ baked amber glass bottle, GCC, from NWQL

\begin{tabular}{|c|c|c|c|}
\hline $\begin{array}{l}\text { Lab. } \\
\text { code }\end{array}$ & $\begin{array}{c}\text { Parameter } \\
\text { code }\end{array}$ & $\begin{array}{l}\text { CAS } \\
\text { number }\end{array}$ & Compound name \\
\hline 09 & $39742 \mathrm{~B}$ & 93765 & $2,4,5-T$ \\
\hline 5408 & $39732 \mathrm{~B}$ & 94757 & 2,4-D \\
\hline 5407 & 38746 A & 94826 & 2,4-DB \\
\hline 5410 & $00000 \mathrm{~A}$ & 81335377 & Acifluorfen (Scepter) \\
\hline 5411 & $00000 \mathrm{~A}$ & 116063 & Aldicarb \\
\hline 5413 & $00000 \mathrm{~A}$ & 1646884 & Aldicarb sulfone \\
\hline 5412 & $00000 \mathrm{~A}$ & & Aldicarb sulfoxide \\
\hline 5414 & $38711 \mathrm{~A}$ & 25057890 & Bentazon \\
\hline 5415 & $04029 \mathrm{~A}$ & 314409 & Bromacil \\
\hline 5416 & $00000 \mathrm{~A}$ & 1689992 & Bromoxynil \\
\hline 5417 & $00000 \mathrm{~A}$ & 63252 & Carbaryl(Sevin) \\
\hline 5418 & $00000 \mathrm{~A}$ & 1563662 & Carbofuran \\
\hline 5449 & $00000 \mathrm{~A}$ & & Carbofuran, 3-hydroxy- \\
\hline 5419 & $00000 \mathrm{~A}$ & 133904 & Chloramben (Amiben) \\
\hline 5421 & $00000 \mathrm{~A}$ & 1897456 & Chlorothalonil \\
\hline 5423 & $00000 \mathrm{~A}$ & 57754855 & Clopyralid \\
\hline 5447 & $00000 \mathrm{~A}$ & & Dacthal, Mono-Acid- \\
\hline 5426 & 38442 A & 1918009 & Dicamba \\
\hline 5404 & $00000 \mathrm{~A}$ & 1194656 & Dichlobenil \\
\hline 5401 & $00000 \mathrm{~A}$ & 120365 & Dichlorprop (2,4-DP) \\
\hline 5400 & $00000 \mathrm{~A}$ & 88857 & Dinoseb (DNBP) \\
\hline 5427 & $00000 \mathrm{~A}$ & 330541 & Diuron \\
\hline 5402 & $00000 \mathrm{~A}$ & 534521 & DNOC \\
\hline 5429 & $00000 \mathrm{~A}$ & 66230044 & Esfenvalerate (Asana XL) \\
\hline 5405 & $00000 \mathrm{~A}$ & 101428 & Fenuron \\
\hline 5430 & $38811 \mathrm{~A}$ & 2164172 & Fluometuron \\
\hline 5432 & $38478 \mathrm{~A}$ & 330552 & Linuron \\
\hline 5433 & $38482 \mathrm{~A}$ & 94746 & MCPA \\
\hline 5434 & 38487 A & 94815 & MCPB \\
\hline 5436 & $38501 \mathrm{~A}$ & 2032657 & Methiocarb \\
\hline 5437 & $00000 \mathrm{~A}$ & 16752775 & Methomyl \\
\hline 5438 & $00000 \mathrm{~A}$ & 90153 & 1-Naphthol \\
\hline 5403 & $00000 \mathrm{~A}$ & 555373 & Neburon \\
\hline 5439 & $00000 \mathrm{~A}$ & 27314132 & Norflurazon \\
\hline 5440 & $00000 \mathrm{~A}$ & 19044883 & Oryzalin (Surflan) \\
\hline 5441 & $38866 \mathrm{~A}$ & 23135220 & Oxamyl \\
\hline 5442 & $00000 \mathrm{~A}$ & 1918021 & Picloram \\
\hline 5443 & $00000 \mathrm{~A}$ & 122429 & Propham (IPC) \\
\hline 5450 & 38538 A & 114261 & Propoxur \\
\hline 5444 & $39762 \mathrm{~B}$ & 93721 & Silvex $(2,4,5-T P)$ \\
\hline 5446 & $00000 \mathrm{~A}$ & 55335063 & Triclopyr \\
\hline 5451 & 00000 & & Set number \\
\hline 5452 & & & BDMC surrogate \\
\hline 5453 & & & Toluic acid surrogate \\
\hline
\end{tabular}

MRL $(\operatorname{Lg} / \mathrm{L})$

0.05

.05

.05

.05

.05

.05

.05

.05

.05

.05

.05

.05

.05

.05

.05

.05

.05

.05

.05

.05

.05

.05

.05

.05

.05

.05

.05

.05

.05

.05

.05

.05

.05

.05

.05

.05

.05

.05

.05

.05

.05

\section{SCHEDULE 2051}

Schedule Description: Pesticides in filtered water extracted in field on Carbopak B SPE cartridge and analyzed by HPLC

Sample Requirements: 1 Carbopak B SPE cartridge through which approximately $1 \mathrm{~L}$ of water filtered through a $0.7-\mu \mathrm{m}$ glass fiber depth filter was processed, annotate actual volume on cartridge form, chilled @ 4 deg. C (packed in ice)

Container Requirements: Original plastic container in which cartridge was shipped

Lab. Parameter CAS MRL code code number compound name (ug/L)

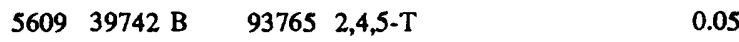

$\begin{array}{lllll}5608 & 39732 & B & 94757 & 2,4-D\end{array}$

$\begin{array}{llll}560738746 \text { A } & 94826 & 2,4-D B & .05\end{array}$

561000000 A 81335377 Acifluorfen (Scepter)

561100000 A 116063 Aldicarb .05

561300000 A 1646884 Aldicarb sulfone

$561200000 \mathrm{~A} \quad$ Aldicarb sulfoxide

561438711 A 25057890 Bentazon

561504029 A 314409 Bromacil .05

561600000 A 1689992 Bromoxynil .05

561700000 A 63252 Carbaryl(Sevin)

561800000 A 1563662 Carbofuran

564900000 A Carbofuran, 3-hydroxy- $\quad .05$

$561900000 \mathrm{~A} \quad 133904$ Chloramben(Amiben) .05

562100000 A 1897456 Chlorothalonil .05

562300000 A 57756855 Clopyralid

564700000 A Dacthal, Mono-Acid-

562638442 A 1918009 Dicamba

560400000 A 1194656 Dichlobenil .05

560100000 A 120365 Dichlorprop (2,4-DP) .05

560000000 A $\quad 88857$ Dinoseb (DNBP)

562700000 A 330561 Diuron

560200000 A 534521 DNOC

562900000 A 66230044 Esfenvalerate (Asana XL) .05

560500000 A 101428 Fenuron

563038811 A 2164172 Fluometuron

$\begin{array}{lll}5632 & 38478 \text { A } 330552 \text { Linuron } & .05\end{array}$

563338482 A 94746 MCPA

563438487 A 94815 MCPB

563638501 A 2032657 Methiocarb

563700000 A 16752775 Methomyl .05

563800000 A 90153 1-Naphthol 05

560300000 A 555373 Neburon $\quad .05$

563900000 A 27314132 Norflurazon $\quad .05$

564000000 A 19044883 Oryzalin (Surflan)

564138866 A 23135220 Oxamyl 05

564200000 A 1918021 Picloram 05

564300000 A 122429 Propham(IPC)

565038538 A 114261 Propoxur 05

$\begin{array}{llll}5644 & 39762 \text { B } 93721 \text { Silvex }(2,4,5-T P) & .05\end{array}$

564600000 A 55335063 Triclopyr

5651 Set number

$5652 \quad$ BDMC surrogate

5653 Toluic acid surrogate 


\section{SCHEDULE 2075}

Schedule Description: Gross organics in surface water for NAWQA

Sample Requirements:100 mL, LC0113 and 1 filter, LC0305, chilled @ 4 deg. C (packed in ice)

Container Requirements: $125-\mathrm{mL}$ amber glass bottle (LC0113), from NWQL. Petri dish (LC0305)

$\begin{array}{rccr}\begin{array}{l}\text { Lab. } \\ \text { code }\end{array} & \begin{array}{c}\text { Parameter } \\ \text { code }\end{array} & \begin{array}{c}\text { CAS } \\ \text { number }\end{array} & \begin{array}{r}\text { Compound name } \\ \text { (ug/L) }\end{array} \\ 113 & 00681 \text { A } & \begin{array}{l}\text { Carbon, organic, dissolved } \\ \text { (DOC) }\end{array} & 0.10 \\ 305 & 00689 \text { A } & \begin{array}{l}\text { Carbon, organic, suspended } \\ \text { (SOC) }\end{array} & .10\end{array}$

\section{SCHEDULE 2085}

Schedule Description: Gross organics in ground water for NAWQA

Sample Requirements: $100 \mathrm{~mL}$, LC0113, chilled @ 4 deg. C (packed in ice)

Container Requirements: $125-\mathrm{mL}$ amber glass bottle (LC0113), from NWQL

$\begin{array}{lccr}\begin{array}{l}\text { Lab. Parameter } \\ \text { code }\end{array} \begin{array}{c}\text { CAS } \\ \text { code }\end{array} & \begin{array}{c}\text { number } \\ \text { Compound name }\end{array} & \begin{array}{r}M R L \\ (\mathrm{ug} / \mathrm{L})\end{array} \\ 113 \text { co681 A } & \begin{array}{c}\text { Carbon, organic, dissolved } \\ \text { (DOC) }\end{array} & 0.10\end{array}$

\section{SCHEDULE 2090}

Schedule Description: Volatile organic compounds at $.2 \mu \mathrm{g} / \mathrm{L}$ by purge and trap GC/MS plus library search of nontarget constituents

Sample Requirements: $3 \times 40-\mathrm{mL}$ vials completely filled to exclude air bubbles, acidified to $\mathrm{pH}<2$ with 2 drops of $1: 1 \mathrm{HCl}$ per vial, and chilled at $4 \mathrm{deg}$. $\mathrm{C}$ (packed in ice). If free chlorine is present, add $25 \mathrm{mg}$ of ascorbic acid to each vial in addition to the $\mathrm{HCl}$.

Container Requirements: $40-\mathrm{mL}$ amber glass septum cap vial (VOC)

\begin{tabular}{|c|c|c|c|c|}
\hline $\begin{array}{l}\text { Lab. } \\
\text { code }\end{array}$ & $\begin{array}{c}\text { Parameter } \\
\text { code }\end{array}$ & $\begin{array}{l}\text { CAS } \\
\text { number }\end{array}$ & Compound name & $\begin{array}{l}\text { MRL } \\
(\mathrm{gg} / \mathrm{L})\end{array}$ \\
\hline 5819 & $34030 \mathrm{C}$ & 71432 & Benzene & \\
\hline 5857 & $77613 \mathrm{C}$ & 87616 & Benzene, 1,2,3-Trichloro- & \\
\hline 5854 & $34551 \mathrm{D}$ & 120821 & Benzene, 1,2,4-Trichloro- & 2 \\
\hline 5846 & $77222 \mathrm{C}$ & 95636 & Benzene, 1,2,4-Trimethyl- & .2 \\
\hline 5852 & $34536 \mathrm{D}$ & 95501 & Benzene, 1,2-Dichloro- & .2 \\
\hline 5860 & $77226 \mathrm{C}$ & 108678 & Benzene, 1,3,5-Trimethyl- & \\
\hline 5849 & $34586 \mathrm{C}$ & 95578 & Benzene, 1,3-Dichloro- & \\
\hline 5850 & $34571 \mathrm{D}$ & 106467 & Benzene, 1,4-Dichloro- & 2 \\
\hline 5844 & $77275 \mathrm{C}$ & 95498 & Benzene, 1-Chloro-2-methyl- & 2 \\
\hline 5858 & $77277 \mathrm{C}$ & 106434 & Benzene, 1-Chloro-4-methyl- & .2 \\
\hline 5839 & $77223 \mathrm{C}$ & 98828 & Benzene, Isopropyl- & .2 \\
\hline 5841 & $81555 \mathrm{C}$ & 108861 & Benzene, Bromo- & .2 \\
\hline 5833 & $34301 \mathrm{C}$ & 108907 & Benzene, Chloro- & .2 \\
\hline 5836 & $81551 \mathrm{C}$ & 1330207 & Benzene, Dimethyl-(Xylene) & .2 \\
\hline 5835 & $34371 \mathrm{C}$ & 100414 & Benzene, Ethyl- & .2 \\
\hline 5848 & $77356 \mathrm{~B}$ & 99036 & Benzene, 1-Methyl-4-isopropyl- & $1-.2$ \\
\hline 5826 & $34010 \mathrm{C}$ & 108883 & Benzene, Methyl-(Toluene) & .2 \\
\hline 5851 & $77342 \mathrm{C}$ & 104518 & Benzene, n-Butyl- & 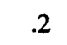 \\
\hline 5843 & $77224 \mathrm{C}$ & 103651 & Benzene, n-Propyl- & .2 \\
\hline 5847 & $77350 \mathrm{C}$ & 135988 & Benzene, sec-Butyl- & .2 \\
\hline 5845 & $77353 \mathrm{C}$ & 98066 & Benzene, tert-Butyl- & .2 \\
\hline 5834 & $77562 \mathrm{C}$ & 630206 & Ethane, 1,1,1,2-Tetrachloro- & .2 \\
\hline 5816 & $34506 \mathrm{C}$ & 71556 & Ethane, 1,1,1-Trichloro- & .2 \\
\hline 5840 & $34516 \mathrm{C}$ & 79345 & Ethane, 1,1,2,2-Tetrachloro- & \\
\hline
\end{tabular}

\section{SCHEDULE 2090--Continued}

Lab. Parameter

code code

$5828 \quad 34511 \mathrm{C}$

$581134496 \mathrm{C}$

$583277651 \mathrm{E}$

$582032103 \mathrm{C}$

$580534311 \mathrm{C}$

$585977652 \mathrm{C}$

$580734501 \mathrm{C}$

$580339175 \mathrm{C}$

$581477093 \mathrm{C}$

$582934475 \mathrm{C}$

$580934546 \mathrm{C}$

$582139180 \mathrm{C}$

$585539702 \mathrm{D}$

$580434413 \mathrm{C}$

$5801377297 \mathrm{C}$

$580234418 \mathrm{C}$

$582430217 \mathrm{C}$

$583132105 \mathrm{C}$

$580834423 \mathrm{C}$

$582332101 \mathrm{C}$

$580134668 \mathrm{C}$

$581732102 \mathrm{C}$

$583832104 \mathrm{C}$

$581532106 \mathrm{C}$

$580634488 \mathrm{C}$

$581078032 \mathrm{C}$

$585634696 \mathrm{D}$

$584277443 \mathrm{C}$

$585382625 \mathrm{C}$

$582234541 \mathrm{C}$

$583077173 \mathrm{C}$

$581277170 \mathrm{C}$

$581877168 \mathrm{C}$

CAS

number Compound name

MRL

(Ug/L)

79005 Ethane, 1,1,2-Trichloro- $\quad 0.2$

75343 Ethane, 1,1-Dichloro .2

106934 Ethane, 1,2-Dibromo-(EDB) $\quad .2$

107062 Ethane, 1,2-Dichloro- $\quad .2$

75003 Ethane, Chloro-

76131 Ethane, Trichlorotrifluoro- $\quad .2$

75354 Ethylene, 1,1-Dichloro $\quad .2$

75014 Ethylene, Chloro(Vinyl Chloride)

156592 Ethylene, cis-1,2-Dichloro- $\quad .2$

127184 Ethylene, Tetrachloro- $\quad .2$

156605 Ethylene, trans-1,2-Dichloro- $\quad .2$

79016 Ethylene, Trichloro- $\quad .2$

87683 Hexachlorobutadiene $\quad .2$

74839 Methane, Bromo $\quad .2$

74975 Methane, Bromochloro- $\quad .2$

74873 Methane, Chloro $\quad .2$

74953 Methane, Dibromo- $\quad .2$

124481 Methane, Dibromochloro- $\quad .2$

75092 Methane, Dichloro- $\quad .2$

75274 Methane, Dichlorobromo- $\quad .2$

75718 Methane, Dichlorodifluoro- $\quad .2$

56235 Methane, Tetrachloro- $\quad .2$

75252 Methane, Tribromo- $\quad .2$

(Bromoform)

67663 Methane, Trichloro- $\quad .2$

(Chlorofom)

75694 Methane, Trichlorofluoro- $\quad .2$

1634044 Propene, 2-methoxy-2-methyl .2

91203 Naphthalene .2

96184 Propane, 1,2,3-Trichloro- $\quad .2$

96128 Propane, 1,2-Dibromo-3- 1 chloro-(DBCP)

78875 Propane, 1,2-Dichloro- $\quad .2$

142289 Propane, 1,3-Dichloro- $\quad .2$

594207 Propane, 2,2-Dichloro- $\quad .2$

582734699 C 10061026 Propene, trans-1,3-Dichloro- $\quad .2$

$583777128 \mathrm{C} \quad 100425$ Styrene 
SCHEDULE 2101

Schedule Description: Organochlorine compounds in biological tissue

Sample Requirements: $500 \mathrm{~g}$ of frozen whole-body biological

sample wrapped in aluminum foil and shipped @ 4 deg. C

(packed in ice)

Container Requirements: Aluminum foil

\begin{tabular}{|c|c|c|c|c|}
\hline $\begin{array}{l}\text { Lab. } \\
\text { code }\end{array}$ & $\begin{array}{l}\text { Parameter } \\
\text { code }\end{array}$ & $\begin{array}{c}\text { CAS } \\
\text { number }\end{array}$ & Compound name & $\begin{array}{c}\text { MRL } \\
(\mu \mathrm{g} / \mathbf{k g})\end{array}$ \\
\hline 7001 & $\overline{00000 \mathrm{~A}}$ & $\overline{--}$ & Chlordane-cis & $\overline{5}$ \\
\hline 7002 & $00000 \mathrm{~A}$ & -- & Chlordane-trans & 5 \\
\hline 7003 & $00000 \mathrm{~A}$ & -- & Dacthal (DCPA) & 5 \\
\hline 7004 & $00000 \mathrm{~A}$ & -- & DDT,o,p' & 5 \\
\hline 7005 & $00000 \mathrm{~A}$ & -- & DDT,p,p' & 5 \\
\hline 7006 & $00000 \mathrm{~A}$ & -- & DDD,p,p' & 5 \\
\hline 7007 & $00000 \mathrm{~A}$ & -- & DDD,o,p' & 5 \\
\hline 7008 & $00000 \mathrm{~A}$ & -- & DDE,o,p' & 5 \\
\hline 7009 & $00000 \mathrm{~A}$ & -- & DDE,p,p' & 5 \\
\hline 7010 & $00000 \mathrm{~A}$ & - & Dieldrin & 5 \\
\hline 7011 & $00000 \mathrm{~A}$ & -- & Endrin & 5 \\
\hline 7012 & $00000 \mathrm{~A}$ & -- & Heptachlor & 5 \\
\hline 7013 & $00000 \mathrm{~A}$ & -- & Heptachlor epoxide & 5 \\
\hline 7014 & $00000 \mathrm{~A}$ & - & Hexachlorobenzene & 5 \\
\hline 7016 & $00000 \mathrm{~A}$ & -- & Alpha-HCH & 5 \\
\hline 7017 & $00000 \mathrm{~A}$ & - & Beta-HCH & 5 \\
\hline 7018 & $00000 \mathrm{~A}$ & -- & Delta-HCH & 5 \\
\hline 7019 & $00000 \mathrm{~A}$ & -- & Gamma-HCH & 5 \\
\hline 7020 & $00000 \mathrm{~A}$ & -- & Methoxychlor,o,p'- & 5 \\
\hline 7021 & $00000 \mathrm{~A}$ & -- & Methoxychlor,p,p'- & 5 \\
\hline 7022 & $00000 \mathrm{~A}$ & -- & Mirex & 5 \\
\hline 7023 & $00000 \mathrm{~A}$ & -- & Nonachlor-cis & 5 \\
\hline 7024 & $00000 \mathrm{~A}$ & -- & Nonachlor-trans & 5 \\
\hline 7025 & $00000 \mathrm{~A}$ & -. & Oxychlordane & 5 \\
\hline 7027 & $00000 \mathrm{~A}$ & -. & Pentachloroanisole & 5 \\
\hline 7028 & $00000 \mathrm{~A}$ & - & Toxaphene & 5 \\
\hline 7029 & $00000 \mathrm{~A}$ & -- & PCB (total) & 5 \\
\hline 7030 & $00000 \mathrm{~A}$ & -- & Aldrin & 5 \\
\hline 7031 & $00000 \mathrm{~A}$ & -- & Lipids \% in tissue & .500 \\
\hline 7032 & $00000 \mathrm{~A}$ & -- & Moisture $\%$ tissue & .500 \\
\hline 7213 & $00000 \mathrm{~A}$ & -- & Hexachlorobutadiene & 5 \\
\hline
\end{tabular}



Table 15.--Radiochemical determinations listed by laboratory code and schedule

\begin{tabular}{|c|c|c|c|c|c|c|c|c|c|c|}
\hline $\mathrm{LC}^{1}$ & $\begin{array}{l}\text { Parameter } \\
\text { code }\end{array}$ & Name, phase, and method & Units & $\begin{array}{l}\text { Reported } \\
\text { as }\end{array}$ & $\begin{array}{l}\text { Volume } \\
\text { needed }\end{array}$ & $\begin{array}{c}\text { Sample } \\
\text { desig- } \\
\text { nation }\end{array}$ & MRL & $\begin{array}{l}\text { 2SPE } \\
\text { LC }\end{array}$ & $\begin{array}{l}\text { 2SPE } \\
\text { PC }\end{array}$ & $\begin{array}{l}\text { 2SPE } \\
\text { MC }\end{array}$ \\
\hline \multicolumn{11}{|c|}{ Gamma scans (2) } \\
\hline 212 & 99451 A & $\begin{array}{l}\text { Gamma scan, BTM, dry wt, gamma } \\
\text { spectroscopy }\end{array}$ & $\mathrm{pCi} / \mathrm{g}$ & -- & $100 \mathrm{~g}$ & CUR & -- & 875 & -- & - \\
\hline 443 & 99452 A & Gamma scan, DIS, gamma spectroscopy & $\mathrm{pCi} / \mathrm{L}$ & -- & $7 \mathrm{~L}$ & FAR & -- & 877 & - & -- \\
\hline 1861 & -- & Gamma scan, SUS, gamma spectroscopy & $\mathrm{pCi} / \mathrm{g}$ & $-\cdot$ & & Filter & -- & 1862 & -- & - \\
\hline \multicolumn{11}{|c|}{ Gross alpha and gross beta } \\
\hline Schedul & e 1131 : Gr & ss alpha and gross beta, bottom material & & & $1 \mathrm{~g}$ & CUR & & & & \\
\hline 1518 & $-\mathbf{A}$ & Gross alpha, BTM, residue procedure & $\mu \mathrm{g} / \mathrm{g}$ & Nat. U & & & 6 & 1519 & 75965 & $\mathbf{A}$ \\
\hline 1520 & $4125 \mathrm{~A}$ & Gross alpha, BTM, residue procedure & $\mathrm{pCi} / \mathrm{g}$ & Th-230 & & & 6 & 1521 & 75955 & $\mathbf{A}$ \\
\hline 1522 & $-\mathbf{A}$ & Gross beta, BTM, residue procedure & $\mathrm{pCi} / \mathrm{g}$ & Cs- 137 & & & 3 & 1523 & -- & \\
\hline 1524 & $4102 \mathrm{~A}$ & Gross beta, BTM, residue procedure & $\mathrm{pCi} / \mathrm{g}$ & $\begin{array}{l}\text { Sr-90/ } \\
\text { Y-90 }\end{array}$ & & & 3 & 1525 & 75966 & $\mathbf{A}$ \\
\hline \multicolumn{5}{|c|}{ Schedule 456 - Gross alpha and gross beta, DIS, field filtered } & $2 \mathrm{~L}$ & FAR & & & & \\
\hline 800 & $80030 \mathrm{~B}$ & Gross alpha, DIS, FF, residue procedure & $\mu g / L$ & Nat. U & & & 3 & 853 & 75986 & B \\
\hline 1397 & $4126 \mathrm{~B}$ & Gross alpha, DIS, FF, residue procedure & $\mathrm{pCi} / \mathrm{L}$ & Th-230 & & & 3 & 1398 & 75987 & B \\
\hline 793 & $80050 \mathrm{~B}$ & Gross beta, DIS, FF, residue procedure & $\mathrm{pCi} / \mathrm{L}$ & $\begin{array}{c}\text { Sr-90/ } \\
\text { Y-90 }\end{array}$ & & & 4 & 855 & 75988 & B \\
\hline 798 & $3515 \mathrm{~B}$ & Gross beta, DIS, FF, residue procedure & $\mathrm{pCi} / \mathrm{L}$ & Cs- 137 & & & 4 & 854 & 75989 & B \\
\hline \multicolumn{4}{|c|}{$\begin{array}{l}\text { Schedule } 458 \text { - Gross alpha and gross beta, field filtered to be } \\
\text { used if solids are higher than } 250 \mathrm{mg} / \mathrm{L}\end{array}$} & & $2 \mathrm{~L}$ & FAR & & & & \\
\hline 1445 & $4126 \mathrm{D}$ & Gross alpha, DIS, FF, HS, co-precipitation & $\mathrm{pCi} / \mathrm{L}$ & Th-230 & & & 3 & 1466 & 75987 & $\mathrm{D}$ \\
\hline 1358 & $80030 \mathrm{D}$ & Gross alpha, DIS, FF, HS, co-precipitation & $\mu g / L$ & Nat. U & & & 3 & 1373 & 75986 & $\mathrm{D}$ \\
\hline 1360 & $3515 \mathrm{D}$ & Gross beta, DIS, FF, HS, co-precipitation & $\mathrm{pCi} / \mathrm{L}$ & Cs-137 & & & 4 & 1375 & 75989 & $\mathrm{D}$ \\
\hline 1359 & $80050 \mathrm{D}$ & Gross beta, DIS, FF, HS, co-precipitation & $\mathrm{pCi} / \mathrm{L}$ & $\begin{array}{l}\text { Sr-90/ } \\
\text { Y-90 }\end{array}$ & & & 4 & 1374 & 75988 & $\mathrm{D}$ \\
\hline \multicolumn{11}{|c|}{ Schedule 165 - Gross alpha and gross beta, suspended } \\
\hline 1852 & -- & Gross alpha, SUS, residue procedure & $\mu \mathrm{g} / \mathrm{g}$ & Nat. U & & Filter & 6 & 1853 & -- & -- \\
\hline 1854 & - & Gross alpha, SUS, residue procedure & $\mathrm{pCi} / \mathrm{g}$ & Th-230 & & Filter & 6 & 1855 & - & -- \\
\hline 1856 & - & Gross beta, SUS, residue procedure & $\mathrm{pCi} / \mathrm{g}$ & Cs-137 & & Filter & 3 & 1857 & -- & -- \\
\hline 1858 & -- & Gross beta, SUS, residue procedure & $\mathrm{pCi} / \mathrm{g}$ & Sr-89/90 & & Filter & 3 & 1859 & -- & -- \\
\hline \multicolumn{11}{|l|}{ Lead } \\
\hline 1182 & $17507 \mathrm{~B}$ & Lead-210, BTM, gamma counting & $\mathrm{pCi} / \mathrm{g}$ & $\mathrm{Pb}-210$ & $100 \mathrm{~g}$ & CUR & .1 & 876 & 75868 & B \\
\hline 1549 & $17507 \mathrm{C}$ & Lead-210, BTM, gamma counting & $\mathrm{pCi} / \mathrm{g}$ & $\mathrm{Pb}-210$ & $100 \mathrm{~g}$ & CUR & 2 & 1550 & 75968 & $\mathbf{C}$ \\
\hline 1503 & $17503 \mathrm{~B}$ & Lead-210, DIS, beta counting & $\mathrm{pCi} / \mathrm{L}$ & $\mathrm{Pb}-210$ & $1 \mathrm{~L}$ & FAR & 1.5 & 1504 & 75995 & B \\
\hline 1547 & 75946 A & Lead-210, SUS, beta counting & $\mathrm{pCi} / \mathrm{g}$ & $\mathrm{Pb}-210$ & $1 \mathrm{~g}$ & SUR & .15 & 1548 & 75949 & A \\
\hline \multicolumn{11}{|c|}{ Polonium } \\
\hline 1545 & 19507 A & Polonium-210, BTM, alpha spectrometry & $\mathrm{pCi} / \mathrm{g}$ & Po-210 & $10 \mathrm{~g}$ & CUR & .1 & 1546 & 4105 & $\mathbf{A}$ \\
\hline 1505 & $19503 \mathrm{~B}$ & Polonium-210, DIS, alpha spectrometry & $\mathrm{pCi} / \mathrm{L}$ & Po-210 & $1 \mathrm{~L}$ & FAR & 1 & 1506 & 75998 & $\mathbf{A}$ \\
\hline 1543 & $75938 \mathrm{~A}$ & Polonium-210, SUS, alpha spectrometry & $\mathrm{pCi} / \mathrm{g}$ & Po-210 & $1 \mathrm{~g}$ & SUR & .1 & 1544 & 75945 & $\mathbf{A}$ \\
\hline
\end{tabular}


Table 15.--Radiochemical determinations listed by laboratory code and schedule --Continued

\begin{tabular}{|c|c|c|c|c|c|c|c|c|c|c|}
\hline$L C^{1}$ & $\begin{array}{l}\text { Parameter } \\
\text { code }\end{array}$ & Name, phase, and method & Units & $\begin{array}{l}\text { Reported } \\
\text { as }\end{array}$ & $\begin{array}{l}\text { Volume } \\
\text { needed }\end{array}$ & $\begin{array}{l}\text { Sample } \\
\text { desig- } \\
\text { nation }^{2}\end{array}$ & MRL & $\begin{array}{l}\text { 2SPE } \\
\text { LC }\end{array}$ & $\begin{array}{l}\text { 2SPE } \\
\text { PC }\end{array}$ & $\begin{array}{l}\text { 2SPE } \\
\text { MC }\end{array}$ \\
\hline \multicolumn{11}{|l|}{ Radium } \\
\hline 1531 & 75944 A & Radium-226, SUS, radon emanation & $\mathrm{pCi} / \mathrm{g}$ & $\mathrm{Ra}-226$ & $1 \mathrm{~g}$ & SUR & 0.1 & 1532 & 75943 & A \\
\hline 794 & $9511 \mathrm{~B}$ & Radium-226, DIS, radon emanation & $\mathrm{pCi} / \mathrm{L}$ & $\mathrm{Ra}-226$ & $1 \mathrm{~L}$ & FAR & .02 & 861 & 76001 & $\mathbf{A}$ \\
\hline 799 & $9510 \mathrm{~B}$ & $\begin{array}{l}\text { Radium-226, DIS, precipitation, planchet } \\
\text { counting }\end{array}$ & $\mathrm{pCi} / \mathrm{L}$ & Ra-226 & $1 \mathrm{~L}$ & FAR & .4 & 864 & 76001 & B \\
\hline 850 & 81366 A & Radium-228, DIS, gamma spectroscopy & $\mathrm{pCi} / \mathrm{L}$ & $\mathrm{Ra}-228$ & $7 \mathrm{~L}$ & FAR & 1 & 866 & 76000 & $\mathbf{A}$ \\
\hline 1364 & $81366 \mathrm{C}$ & $\begin{array}{l}\text { Radium-228, DIS, FF, radiochemical } \\
\text { separation and beta counting }\end{array}$ & $\mathrm{pCi} / \mathrm{L}$ & Ra-228 & $2 \mathrm{~L}$ & FAR & 1 & 1379 & 76000 & C \\
\hline 1533 & 75937 A & Radium-228, SUS, beta counting & $\mathrm{pCi} / \mathrm{g}$ & $\mathbf{R a}-228$ & $1 \mathrm{~g}$ & SUR & .1 & 1534 & 75948 & A \\
\hline \multicolumn{4}{|c|}{ Schedule 1136 - Rad hum, bottom material } & $100 \mathrm{~g}$ & CUR & & & & & \\
\hline 1528 & $9507 \mathrm{~B}$ & Radium-226, BTM, gamma counting & $\mathrm{pCi} / \mathrm{g}$ & $\mathrm{Ra}-226$ & & & .4 & 1529 & 4107 & $\mathbf{B}$ \\
\hline 1526 & 75977 A & Radium-228, BTM, gamma counting & $\mathrm{pCi} / \mathrm{g}$ & Ra-228 & & & .8 & 1527 & 4106 & A \\
\hline \multicolumn{11}{|l|}{ Rad on } \\
\hline 490 & $82305 \mathrm{~A}$ & Radon-222, DIS, radon emanation & $\mathrm{pCi} / \mathrm{L}$ & Rn-222 & $50 \mathrm{~mL}$ & Bubbler & .2 & 865 & 76002 & A \\
\hline 1369 & 82303 B & Radon-222, DIS, liquid scintillation & $\mathrm{pCi} / \mathrm{L}$ & $R n-222$ & & Call lab. & 80 & 1384 & 76002 & B \\
\hline \multicolumn{11}{|c|}{ Strontium } \\
\hline 795 & $13503 \mathrm{~B}$ & $\begin{array}{l}\text { Strontium- } 90, \text { DIS, chemical separation and } \\
\text { beta counting }\end{array}$ & $\mathrm{pCi} / \mathrm{L}$ & Sr-90 & $1 \mathrm{~L}$ & FAR & .5 & 873 & 76003 & B \\
\hline \multicolumn{11}{|c|}{ Thorium } \\
\hline \multicolumn{5}{|c|}{ Schedule 1141 - Thorium, bottom material } & $10 \mathrm{~g}$ & CUR & & & & \\
\hline 1537 & 26507 A & Thorium-230, BTM, alpha spectrometry & $\mathrm{pCi} / \mathrm{g}$ & Th-230 & & & .1 & 1538 & 4108 & A \\
\hline 1535 & 26631 A & Thorium-232, BTM, alpha spectrometry & $\mathrm{pCi} / \mathrm{g}$ & Th-232 & & & .1 & 1536 & 4110 & A \\
\hline \multicolumn{5}{|c|}{ Schedule 1139 - Thorium, dissolved } & $1 \mathrm{~L}$ & FAR & & & & \\
\hline 142 & $26503 \mathrm{~B}$ & Thorium-230, DIS, alpha spectrometry & $\mathrm{pCi} / \mathrm{L}$ & Th-230 & & & 1 & 1473 & 75997 & B \\
\hline 1501 & 75976 A & Thorium-232, DIS, alpha spectrometry & $\mathrm{pCi} / \mathrm{L}$ & Th-232 & & & 1 & 1502 & 75999 & A \\
\hline \multicolumn{5}{|c|}{ Schedule 1140 - Thorium, su spended } & $1 \mathrm{~g}$ & SUR & & & & \\
\hline 1541 & 75939 A & Thorium-230, SUS, alpha spectrometry & $\mathrm{pCi} / \mathrm{g}$ & Th-230 & & & .1 & 1542 & 75952 & A \\
\hline 1539 & $75953 \mathrm{~A}$ & Thorium-232, SUS, alpha spectrometry & $\mathrm{pCi} / \mathrm{g}$ & Th-232 & & & .1 & 1540 & 75936 & $\mathbf{A}$ \\
\hline \multicolumn{11}{|l|}{ Tritium } \\
\hline 452 & $7000 \mathrm{D}$ & Tritium,WWR, liquid scintillation & $\mathrm{pCi} / \mathrm{L}$ & $\mathrm{H}-3$ & $25 \mathrm{~mL}$ & LC0452 & 26 & 879 & 75985 & D \\
\hline 460 & $7000 \mathrm{~A}$ & $\begin{array}{l}\text { Tritium, WWR, electrolytic enrichment, } \\
\text { liquid scintillation }\end{array}$ & $\mathrm{pCi} / \mathrm{L}$ & H-3 & $250 \mathrm{~mL}$ & LC0460 & 5.7 & 882 & 75985 & A \\
\hline 1043 & $7000 \mathrm{~B}$ & $\begin{array}{l}\text { Tritium, WWR, electrolytic enrichment, } \\
\text { gas counting }\end{array}$ & $\mathrm{pCi} / \mathrm{L}$ & $\mathrm{H}-\mathbf{3}$ & $1 \mathrm{~L}$ & LC1043 & .3 & 883 & 75985 & B \\
\hline 624 & $7000 \mathrm{E}$ & $\begin{array}{l}\text { Tritium, WWR, electrolytic enrichment, } \\
\text { gas counting }\end{array}$ & $\mathrm{pCi} / \mathrm{L}$ & $\mathrm{H}-3$ & $500 \mathrm{~mL}$ & LC0624 & 2.5 & 1000 & 75985 & $\mathrm{E}$ \\
\hline 1565 & $7000 \mathrm{~F}$ & $\begin{array}{l}\text { Tritium, WWR, electrolytic enrichment, } \\
\text { liquid scintillation }\end{array}$ & $\mathrm{pCi} / \mathrm{L}$ & H-3 & $1 \mathrm{~L}$ & LC1565 & 1 & 1566 & 75985 & $\mathbf{F}$ \\
\hline \multicolumn{11}{|c|}{ Uranium } \\
\hline 1006 & $22703 \mathrm{H}$ & Uranium, DIS, FF, fluorometry & $\mu g / L$ & $\mathbf{U}$ & $1 \mathrm{~L}$ & FAR & 1 & 1007 & $75900 \mathrm{H}$ & $\mathrm{H}$ \\
\hline 1386 & $22703 \mathrm{E}$ & Uranium, DIS, FF, laser phosphorescence & $\mu g / L$ & $\mathbf{U}$ & $1 \mathrm{~L}$ & FAR & .01 & 1390 & $75990 \mathrm{E}$ & $\mathrm{E}$ \\
\hline 1385 & $22703 \mathrm{C}$ & Uranium, DIS, FF, laser phosphorescence & $\mu \mathrm{g} / \mathrm{L}$ & $\mathbf{U}$ & $1 \mathrm{~L}$ & FAR & .4 & 1389 & $75990 \mathrm{C}$ & C \\
\hline
\end{tabular}


Table 15.--Radiochemical determinations listed by laboratory code and schedule --Continued

\begin{tabular}{|c|c|c|c|c|c|c|c|c|c|c|}
\hline$L C^{1}$ & $\begin{array}{l}\text { Parameter } \\
\text { code }\end{array}$ & Name, phase, and method & Units & $\begin{array}{c}\text { Reported } \\
\text { as }\end{array}$ & $\begin{array}{l}\text { Volume } \\
\text { needed }\end{array}$ & $\begin{array}{l}\text { Sample } \\
\text { desig- } \\
\text { nation }{ }^{2}\end{array}$ & MRL & $\begin{array}{c}\text { 2SPE } \\
\text { LC }\end{array}$ & $\begin{array}{l}\text { 2SPE } \\
\text { PC }\end{array}$ & $\begin{array}{l}2 \mathrm{SPE} \\
\text { MC }\end{array}$ \\
\hline \multicolumn{5}{|c|}{ Schedule 1138-Uranium, BTM } & $100 \mathrm{~g}$ & CUR & & & & \\
\hline 1509 & 28014 A & Uranium-234, BTM, alpha spectrometry & $\mathrm{pCi} / \mathrm{g}$ & U-234 & & & 1 & 1510 & 4111 & A \\
\hline 1515 & 22612 A & Uranium-235, BTM, alpha spectrometry & $\mathrm{pCi} / \mathrm{g}$ & U-235 & & & & 1516 & 4112 & $\mathbf{A}$ \\
\hline 1511 & 28016 A & Uranium-238, BTM, alpha spectrometry & $\mathrm{pCi} / \mathrm{g}$ & U-238 & & & 1 & 1516 & 75962 & $\mathbf{A}$ \\
\hline
\end{tabular}

\section{Schedule 1130 - Uranium, DIS, field filtered}

136622610 A Uranium-234, DIS, alpha spectrometry

136722620 A Uranium-235, DIS, alpha spectrometry

136822603 A Uranium-238, DIS, alpha spectrometry

Schedule 1137 - Urantum, suspended

147475942 A Uranium-234, SUS, alpha spectrometry

147675975 A Uranium-235, SUS, alpha spectrometry

150775940 A Uranium-238, SUS, alpha spectrometry

Schedules containing various radiochemical determinations

Schedule 473 - Radium-226 and Uranium, field filtered

7949511 B Radium-226, DIS, FF, radon emanation

$138522703 \mathrm{C}$ Uranium, DIS, FF, laser phosphorescence

Schedule 308 - Gross alpha and gross beta plus radium and uranium; DIS, field filtered

80080030 B Gross alpha, DIS, FF, residue procedure

13974126 B Gross alpha, DIS, FF, residue procedure

79380050 B Gross beta, DIS, FF, residue procedure

7983515 B Gross beta, DIS, FF, residue procedure

7949511 B Radium-226, DIS, FF, radon emanation

138622703 E Uranium, DIS, FF, laser phophorescence
$\mathrm{PCi} / \mathrm{L} \quad \mathrm{U}-234$

$\mathrm{PCi} / \mathrm{L} \quad \mathrm{U}-235$

$\mathrm{pCi} / \mathrm{L} \quad \mathrm{U}-238$

$\mathrm{pCi} / \mathrm{g} \quad \mathrm{U}-234$

pCi/g U-235

pCi/g U-238

$\mathrm{pCi} / \mathrm{L} \quad \mathrm{Th}-230$

pCi/L Sr-90/

$\mathrm{pCi} / \mathrm{L} \quad \mathrm{Cs}-137$

$\mathrm{pCi} / \mathrm{L} \quad \mathrm{Ra}-226$

Schedule 1703 - Radhum.226 and Uranium, field filtered (NASQAN)

7949511 B Radium-226, DIS, FF, radon emanation

$\mathrm{pCi} / \mathrm{L} \quad \mathrm{Ra}-226$

138622703 E Uranium, DIS, FF, laser phophorescence

$\mu \mathrm{g} / \mathrm{L} \quad \mathrm{U}$

Schedule 214 - Gross alpha and gross beta, field filtered;

Radiu $m$ and uranium, field filtered

800 80030 B Gross alpha, DIS, FF, residue procedure

13974126 B Gross alpha, DIS, FF, residue procedure

79380050 B Gross beta, DIS, FF, residue procedure

$\mu \mathrm{g} / \mathrm{L} \quad$ Nat. U

$\mathrm{pCi} / \mathrm{L} \quad \mathrm{Th}-230$

pCi/L Sr-90/

7983515 B Gross beta, DIS, FF, residue procedure

$\mathrm{pCi} / \mathrm{L} \quad \mathrm{Cs}-137$

7999510 B Radium-226, DIS, FF, precipation, planchet pCi/L Ra-226 counting

100622703 H Uranium, DIS, FF, fluorometry
PCi/L Ra-226

$\mu \mathrm{g} / \mathrm{L} \quad \mathrm{U}$

$\mu \mathrm{g} / \mathrm{L} \quad$ Nat. $\mathrm{U}$ Y -90

$\mu \mathrm{g} / \mathrm{L} \quad \mathrm{U}$

Y-90

2L FAR

$\begin{array}{llll}.1 & 1381 & 75992 & \text { A } \\ .1 & 1382 & 75994 & \text { A } \\ .1 & 1383 & 75991 & \text { A }\end{array}$

$1 \mathrm{~g}$ SUR

$1 \mathrm{~g}$ SUR

$\begin{array}{lrrr}.02 & 861 & 76001 & \text { A } \\ .4 & 1389 & 75990 & \text { C }\end{array}$

4 L FAR

$\begin{array}{lrrr}3 & 853 & 75986 \mathrm{~B} & \text { B } \\ 3 & 1398 & 75987 \mathrm{~B} & \text { B } \\ 4 & 854 & 75988 \mathrm{~B} & \text { B } \\ & & & \\ 4 & 854 & 75989 \mathrm{~B} & \text { B } \\ .02 & 861 & 76001 \mathrm{~A} & \text { A } \\ .01 & 1390 & 75990 \mathrm{E} & \text { E }\end{array}$

2L FAR

$\begin{array}{rrrr}.02 & 861 & 76001 & \text { A } \\ .01 & 1390 & 75990 & \text { E }\end{array}$

4L FAR

$\mu \mathrm{g} / \mathrm{L}$

75941 A

75947 A

4113 A

$\begin{array}{lll}.1 & 1508 & 4113\end{array}$

A


Table 15.--Radiochemical determinations listed by laboratory code and schedule --Continued

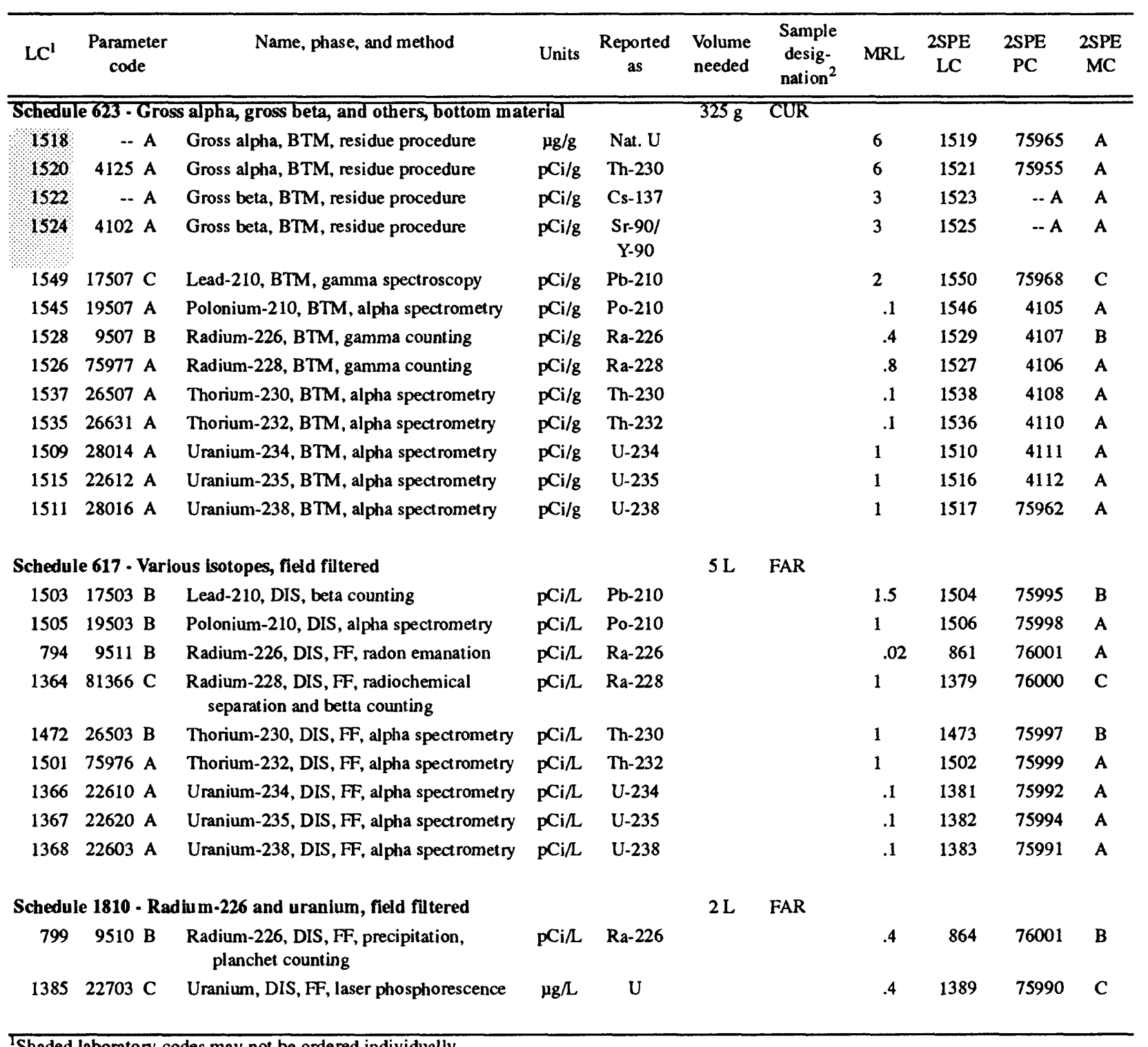

Shaded laboratory codes may not be ondered individually.

${ }^{2}$ See table 4. 
Table 16.--Stable isotope ratios listed by laboratory code and schedule

\begin{tabular}{|c|c|c|c|c|c|c|c|}
\hline $\begin{array}{l}\text { Lab. } \\
\text { code }^{1}\end{array}$ & $\begin{array}{l}\text { Para- } \\
\text { meter } \\
\text { code }\end{array}$ & Compound name & $\begin{array}{l}\text { Unit of } \\
\text { measure- } \\
\text { ment }\end{array}$ & $\begin{array}{l}\text { Preferred sample } \\
\text { requirements }\end{array}$ & $\begin{array}{l}\text { Minimum sample } \\
\text { requirements }\end{array}$ & $\begin{array}{l}\text { Label } \\
\text { sample }\end{array}$ & $\begin{array}{l}\text { Pre- } \\
\text { cision } \\
\pm\end{array}$ \\
\hline \multicolumn{8}{|c|}{ Carbon } \\
\hline 1243 & 99481 & $\begin{array}{l}\text { A Carbon-13/Carbon-12 and } \\
\text { Oxygen-18/Oxygen-16 }\end{array}$ & & $100 \mu \mathrm{mol}$ & $40 \mu \mathrm{mol}$ & LC1243 & 0.2 \\
\hline 1135 & 82339 & A Carbon-13/Carbon-12, carbonate rock & $\begin{array}{l}\text { Per mil } \\
\text { relative to } \\
\text { PDB }\end{array}$ & $\begin{array}{l}1 \mathrm{~g} \text { pure } \\
\text { calcite }\end{array}$ & $\begin{array}{l}4 \mathrm{mg} \text { pure } \\
\text { calcite }\end{array}$ & LC1135 & .3 \\
\hline 1205 & & $\begin{array}{l}\text { A Carbon-13/Carbon-12, organic soil or } \\
\text { rock material }\end{array}$ & $\begin{array}{l}\text { Per mil } \\
\text { relative to } \\
\text { PDB }\end{array}$ & $\begin{array}{l}1 \mathrm{~g} \\
\text { carbon }\end{array}$ & $0.5 \mathrm{mg}$ carbon & LC1205 & .3 \\
\hline 440 & 82081 & $\begin{array}{l}\text { A Carbon-13/Carbon-12, whole water, } \\
\text { 1-L glass bottle }\end{array}$ & $\begin{array}{l}\text { Per mil } \\
\text { relative to } \\
\text { PDB }\end{array}$ & $\begin{array}{l}250 \mathrm{mg} \text { of } \mathrm{SrCO}_{3} \\
\text { precipitate }\end{array}$ & $\begin{array}{l}50 \mathrm{mg} \text { of } \mathrm{SrCO}_{3} \\
\text { precipitate }\end{array}$ & LC440 & .3 \\
\hline 1244 & - & $\begin{array}{l}\text { Carbon-13/Carbon-12, gaseous } \mathrm{CO}_{2} \text {. } \\
\text { Use } 6 \text { or 9-mm O.D. Pyrex tubes }\end{array}$ & $\begin{array}{l}\text { Per mil } \\
\text { relative to } \\
\text { PDB }\end{array}$ & $100 \mu \mathrm{mol}$ & $40 \mu \mathrm{mol}$ & LC1244 & .3 \\
\hline 1198 & 82172 & A Carbon-14, aqueous percent modern & Percent & $\begin{array}{l}220 \text { L RUR } \\
\text { (see table 4) }\end{array}$ & $\begin{array}{l}\text { Sufficient sample } \\
\text { to yield } 2 \mathrm{~g} \text { ele- } \\
\text { mental } \mathrm{C} \text { when } \\
\text { precipitated }\end{array}$ & LC1198 & - \\
\hline 1199 & 82172 & $\begin{array}{l}\text { B Carbon-14 (Field precipitated with } \\
\text { carbon free reagents) }\end{array}$ & Percent & 5 g elemental $\mathrm{C}$ & 2 g elemental $\mathrm{C}$ & LC1199 & -- \\
\hline \multicolumn{8}{|c|}{ Deuterium } \\
\hline 1574 & 82082 & B Deuterium/protium, aqueous & $\begin{array}{l}\text { Per mil } \\
\text { relative to } \\
\text { SMOW }\end{array}$ & $60 \mathrm{~mL}$ & $5 \mathrm{~mL}$ & LC1574 & 2 \\
\hline \multicolumn{8}{|c|}{ Nitrogen } \\
\hline 1717 & 82691 & $\begin{array}{l}\text { A Nitrogen-15/Nitrogen-14 ratio, DIS } \\
\text { as ammonia }\end{array}$ & $\begin{array}{l}\text { Per mil rela- } \\
\text { tive to air }\end{array}$ & $\begin{array}{l}\text { Sufficient sample } \\
\text { to yield } 1 \mathrm{~g} \mathrm{~N}\end{array}$ & $\begin{array}{l}\text { Sufficient sample } \\
\text { to yield } 1 \mathrm{mg} \mathrm{N}\end{array}$ & LC1717 & .2 \\
\hline 1718 & 82690 & $\begin{array}{l}\text { A Nitrogen-15/Nitrogen-14 ratio, DIS } \\
\text { as nitrate }\end{array}$ & $\begin{array}{l}\text { Per mil rela- } \\
\text { tive to air }\end{array}$ & $\begin{array}{l}\text { Sufficient sample } \\
\text { to yield } 1 \mathrm{~g} \mathrm{~N}\end{array}$ & $\begin{array}{l}\text { Sufficient sample } \\
\text { to yield } 1 \mathrm{mg} \mathrm{N}\end{array}$ & LC1718 & \\
\hline 1719 & 82688 & $\begin{array}{l}\text { A Nitrogen-15/Nitrogen-14 ratio, solid } \\
\text { as nitrate }\end{array}$ & $\begin{array}{l}\text { Per mil rela- } \\
\text { tive to air }\end{array}$ & $\begin{array}{l}\text { Sufficient sample } \\
\text { to yield } 1 \mathrm{~g} \mathrm{~N}\end{array}$ & $\begin{array}{l}\text { Sufficient sample } \\
\text { to yield } 1 \mathrm{mg} \mathrm{N}\end{array}$ & LC1719 & \\
\hline 1720 & 82689 & $\begin{array}{l}\text { A Nitrogen-15/Nitrogen-14 ratio, solid } \\
\text { as ammonia }\end{array}$ & $\begin{array}{l}\text { Per mil rela- } \\
\text { tive to air }\end{array}$ & $\begin{array}{l}\text { Sufficient sample } \\
\text { to yield } 1 \mathrm{~g} \mathrm{~N}\end{array}$ & $\begin{array}{l}\text { Sufficient sample } \\
\text { to yield } 1 \mathrm{mg} \mathrm{N}\end{array}$ & LC1720 & \\
\hline 1921 & & $\begin{array}{l}\text { Nitrogen-15/Nitrogen-14 ratio, DIS } \\
\text { as nitrate and ammonia combined }\end{array}$ & $\begin{array}{l}\text { Per mil rela- } \\
\text { tive to air }\end{array}$ & $\begin{array}{l}\text { Sufficient sample } \\
\text { to yield } 1 \mathrm{~g} \mathrm{~N}\end{array}$ & $\begin{array}{l}\text { Sufficient sample } \\
\text { to yield } 1 \mathrm{mg} \mathrm{N}\end{array}$ & LC1921 & \\
\hline \multicolumn{8}{|c|}{ Oxygen } \\
\hline 489 & 82085 & A Oxygen-18/Oxygen-16, aqueous & $\begin{array}{l}\text { Per mil } \\
\text { relative to } \\
\text { SMOW }\end{array}$ & $60 \mathrm{~mL}$ & $5 \mathrm{~mL}$ & LC0489 & .2 \\
\hline 1137 & 82337 & A Oxygen-18/Oxygen-16, rock & $\begin{array}{l}\text { Per mil } \\
\text { relative to } \\
\text { SMOW }\end{array}$ & $1 \mathrm{~g}$ pure calcite & $4 \mathrm{mg}$ pure calcite & LC1137 & .2 \\
\hline
\end{tabular}


Table 16.--Stable isotope ratios listed by laboratory code and schedule -.-Continued

\begin{tabular}{|c|c|c|c|c|c|c|c|}
\hline $\begin{array}{l}\text { Lab. } \\
\text { code }^{i}\end{array}$ & $\begin{array}{l}\text { Para- } \\
\text { meter } \\
\text { code }\end{array}$ & Compound name & $\begin{array}{c}\text { Unit of } \\
\text { measure- } \\
\text { ment }\end{array}$ & $\begin{array}{l}\text { Preferred sample } \\
\text { requirements }\end{array}$ & $\begin{array}{l}\text { Minimum sample } \\
\text { requirements }\end{array}$ & $\begin{array}{l}\text { Label } \\
\text { sample }\end{array}$ & $\begin{array}{l}\text { Pre- } \\
\text { cision } \\
\pm\end{array}$ \\
\hline \multicolumn{8}{|l|}{$\overline{\text { Sulfur }}$} \\
\hline 1951 & - & $\begin{array}{l}\text { Sulfur-34/Sulfur-32, as sulfate, } \\
\text { aqueous (high concentration) }\end{array}$ & Per mil & -- & $30 \mathrm{mg} \mathrm{SO} 4$ & - & 0.5 \\
\hline 1949 & - & $\begin{array}{l}\text { Sulfur-34/Sulfur-32, as sulfate, } \\
\text { aqueous (low concentration) }\end{array}$ & Per mil & -- & $\underset{\text { resin }}{30 \mathrm{mg} \mathrm{SO}_{4} \text { on }}$ & -- & .5 \\
\hline 1948 & - & $\begin{array}{l}\text { Sulfur-34/Sulfur-32, as sulfide, } \\
\text { aqueous }\end{array}$ & Per mil & -- & $30 \mathrm{mg} \mathrm{Ag}_{2} \mathrm{~S}$ & -- & .5 \\
\hline 1950 & - & Sulfur-34/Sulfur-32, as sulfate, rock & Per mil & -- & $30 \mathrm{mg} \mathrm{SO}_{4}$ & -- & .5 \\
\hline 1947 & - & Sulfur-34/Sulfur-32, as sulfide, rock & Per mil & - & $30 \mathrm{mg} \mathrm{Ag} 2 \mathrm{~S}$ & -- & .5 \\
\hline \multicolumn{8}{|c|}{ Schedule 921} \\
\hline 1952 & - & Sulfur-34/Sulfur-32, as sulfate, rock & Per mil & - & $30 \mathrm{mg} \mathrm{SO}_{4}$ & -- & .5 \\
\hline 1953 & - & Sulfur-34/Sulfur-32, as disulfide, rock & Per mil & - & $30 \mathrm{mg} \mathrm{Ag}_{2} \mathrm{~S}$ & -- & .5 \\
\hline 1954 & - & $\begin{array}{l}\text { Sulfur-34/Sulfur-32, as monosulfide, } \\
\text { rock }\end{array}$ & Per mil & - & $30 \mathrm{mg} \mathrm{Ag} \mathrm{S}$ & -- & .5 \\
\hline \multicolumn{3}{|c|}{ Schedule 1142} & & $60 \mathrm{~mL}$ & $5 \mathrm{~mL}$ & SC1142 & \\
\hline 489 & $82085 \mathrm{~A}$ & $\begin{array}{l}\text { Oxygen-18/Oxygen-16 ratio, } \\
\text { aqueous }\end{array}$ & $\begin{array}{l}\text { Per mil rela- } \\
\text { tive to } \\
\text { SMOW }\end{array}$ & & & & .2 \\
\hline 1574 & 82082 B & Deuterium/protium, aqueous & $\begin{array}{l}\text { Per mil rela- } \\
\text { tive to } \\
\text { SMOW }\end{array}$ & & & & 2 \\
\hline
\end{tabular}

Shaded laboratory codes may not be ordered individually. 


\section{SELECTED REFERENCES}

American Society for Testing and Materials, 1993, Annual book of ASTM standards, Section 11, Water and environmental technology: v. 11.01, p. 5.

Barnett, P.R., and Mallory, E.C., Jr., 1971, Determination of minor elements in water by emission spectroscopy: U.S. Geological Survey Techniques of Water-Resources Investigations, book 5, chap. A2, 31 p.

Brenton, R.W., and Arnett, TL., 1993, Methods of analysis by the U.S. Geological Survey National Water Quality Laboratory--Determination of dissolved organic carbon by UV-promoted persulfate oxidation and infrared spectrometry: U.S. Geological Survey Open-File Report 92-480, 12 p.

Britton, L.J., and Greeson, P.E., eds., 1989, Methods for collection and analysis of aquatic biological and microbiological samples: U.S. Geological Survey Techniques of Water-Resources Investigations, book 5, chap. A4, 363 p.

Brown, G.E., and McLain, B.J., 1994, Methods of analysis by the U.S. Geological Survey National Water Quality Laboratory--Determination of antimony by automated-hydride atomic absorption spectrophotometry: U.S. Geological Survey Open-File Report 93-664, 17 p.

Damrau, D.L., 1993, Methods of analysis by the U.S. Geological Survey National Water Quality Laboratory-Determination of low-level silver by graphite furnace atomic absorption spectrophotometry: U.S. Geological Survey Open-File Report 93-416, 14 p.

Faires, L.M., 1993, Methods of analysis by the U.S. Geological Survey National Water Quality Laboratory-Determination of metals in water by inductively coupled plasma-mass spectrometry: U.S. Geological Survey Open-File Report 92-634, 28 p.

Fishman, M.J., and Friedman, L.C., eds., 1989, Methods for determination of inorganic substances in water and fluvial sediments: U.S. Geological Survey Techniques of Water-Resources Investigations, book 5, chap. Al, $545 \mathrm{p}$.

Fishman, M.J., ed., 1993, Methods of analy sis by the U.S. Geological Survey National Water Quality Laboratory-Determination of inorganic and organic constituents in water and fluvial sediments: U.S. Geological Survey Open-File Report 93-125, 217 p.

Friedman, L.C., and Erdmann, D.E., 1982, Quality assurance practices for the chemical and biological analyses of water and fluvial sediments: U.S. Geological Survey Techniques of Water-Resources Investigations, book 5, chap. A6, $181 \mathrm{p}$.

Greeson, P.E., ed., 1979, A supplement to--Methods for collection and analysis of aquatic biological and microbiological samples (U.S. Geological Survey Techniques of Water-Resources Investigations, book 5, chap. A4): U.S. Geological Survey Open-File Report 79-1279, 96 p.

Lindley, C.E., Burkhardt, M.R., and DeRusseau, S.N., 1994, Methods of analysis by the U.S. Geological Survey National Water Qual ity Laboratory--Extraction of nitroaromatic compounds from water by polystyrene divinylbenzene cartridge and determination by high-performance liquid chromatography: U.S. Geological Survey Open-File Report 94-62, 15 p.

Markovchick, D.J., Lewis, J.A., Brenton, R.W., Iverson, J.L., and Wharry, H.L., 1994, Methods of analysis by the U.S. Geological Survey National Water Quality Laboratory--Determination of triazine and other nitrogencontaining compounds by gas chromatography with nitrogen phosphorus detectors: U.S. Geological Survey Open-File Report 94-37, 17 p.

McLain, B.J., 1993, Methods of analysis by the U.S. Geological Survey National Water Quality Laboratory--Determination of chromium in water by graphite furnace atomic absorption spectrophotometry: U.S. Geological Survey Open-File Report 93-449, 16 p. 
Patton, C.J., and Truitt, E.P., 1992, Methods of analysis by the U.S. Geological Survey National Water Quality Laboratory--Determination of total phosphorus by a Kjeldahl digestion method and an automated colorimetric finish that includes dialysis: U.S. Geological Survey Open-File Report 92-146, 39 p.

Sandstrom, M.W., Wydoski, D.S., Schroeder, M.P., Zamboni, J.L., and Foreman, W.T., 1994, Methods of analysis by the U.S. Geological Survey National Water Quality Laboratory--Determination of organonitrogen herbicides in water by solid-phase extraction and capillary-column gas chromatography/mass spectrometry with selected-ion monitoring: U.S. Geological Survey Open-File Report 91-519, 26 p.

Thatcher, L.L., Janzer, V.J., and Edwards, K.W., 1977, Methods for determination of radioactive substances in water and fluvial sediments: U.S. Geol ogical Survey Techniques of Water-Resources Investigations, book 5, chap. A5, 95 p.

U.S. Environmental Protection Agency, 1983, Methods for chemical analysis of water and wastes: Cincinnati, Ohio, Environmental Monitoring and Support Laboratory, EPA-600/4-79-020, March 1983, variable pagination.

1988, Methods for determination of organic compounds in drinking water: Cincinnati, Ohio, Environmental Monitoring Systems Laboratory, EPA-600/4-88-039, December 1988, 125 p.

1992, Protection of environment: Method 608--Organochlorine pesticides and PCBs: U.S. Code of Federal Regulations, Title 40, Part 136, Appendix A, July 1, 1992, p. 409-429.

1993, Maximum contaminant levels (subpart B of part 141, National primary drinking-water regulations): U.S. Code of Federal Regulations, Title 40, Parts 141-143, revised as of June 1993, p. 596.

Warner, S.L., and Johnson, S.M., 1994, Methods of analy sis by the U.S. Geological Survey National Water Quality Laboratory--Determination of selected carbamate pesticides in water by high-performance liquid chromatography: U.S. Geological Survey Open-File Report 93-650, 29 p.

Wershaw, R.L., Fishman, M.J., Grabbe, R.R., and Lowe, L.E., eds., 1987, Methods for the determination of organic substances in water and fluvial sediments: U.S. Geological Survey Techniques of Water-Resources Investigations, book 5, chap. A3, $80 \mathrm{p}$. 
Districts and NAWQA study units are urged to have at least one of their members at each site maintain guest privileges on the NWQL partition in order to have access to the Schedules, Parameters, and Network (SPN) program. Guest privileges can be arranged by request to DENADP by means of EDOC.

SPN is invoked by typing "SPN" at the "OK" prompt on the Prime: OK, SPN

******Welcome to the SPN Menu******
Which year would you like to work in:
1. 1992
2. 1993
3. 1994
4. EXIT the SPN Menu
Please enter your selection:

Previous year's entries (1992 and 1993) offer a computer-accessible, historical record of the final disposition of the catalog's contents for those fiscal years. No addendum to the printed catalog will be offered to supply this information. The current fiscal year's entries are al ways up-to-date as to availability and price. Entering " 3 " brings up this screen:

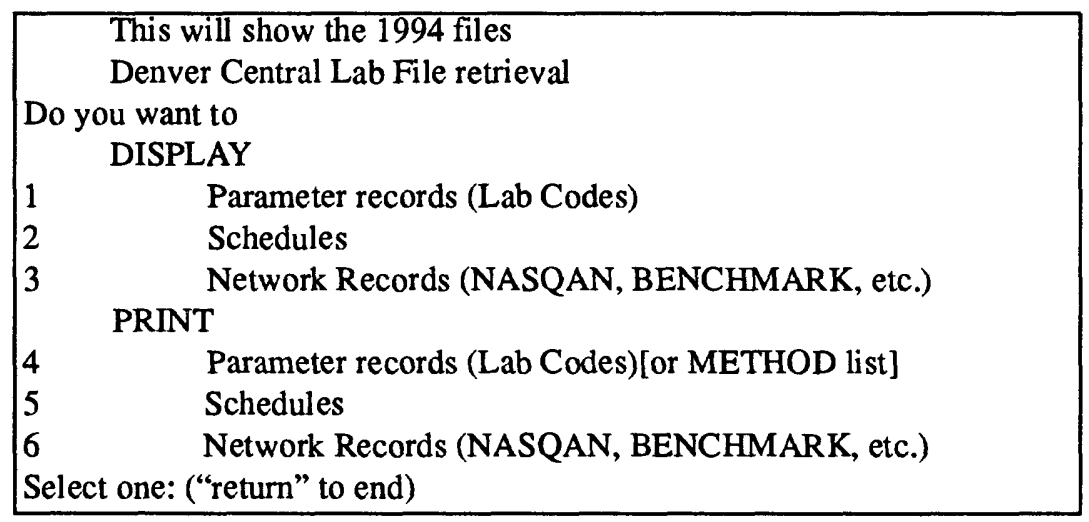

Options 1 and 4, respectively, will display on the screen or print results of the following retrievals based on the options of the following screen:

\begin{tabular}{|ll|}
\hline \multicolumn{2}{|c|}{ Denver Central Lab Parameter Code Dictionary } \\
Do you want to retrieve by: \\
$1 \quad$ Lab Code \\
$2 \quad$ Watstore Code \\
3 & Name Fragment \\
Select one: ("return" to end)
\end{tabular}

These retrievals will list short and long names, present price, WATSTORE (Water Data Storage and Retrieval System) code, reporting units, and minimum reporting limits. Also, method code, container and volume requirements, and other details are reported. Selecting option 4 will print to the Laboratory printer or to a file in a depot. Consult your local system manager for information on the use and retrieval of results from your local Prime depot.

NOTE: Custom analyses or schedules under development will appear as laboratory codes, usually in the 8000 series. Since a schedule is a collection of laboratory codes, most of the information (except price) will be blank or meaningless. 
Options 2 or 5 will present information on laboratory schedules based on the following retrieval criteria:

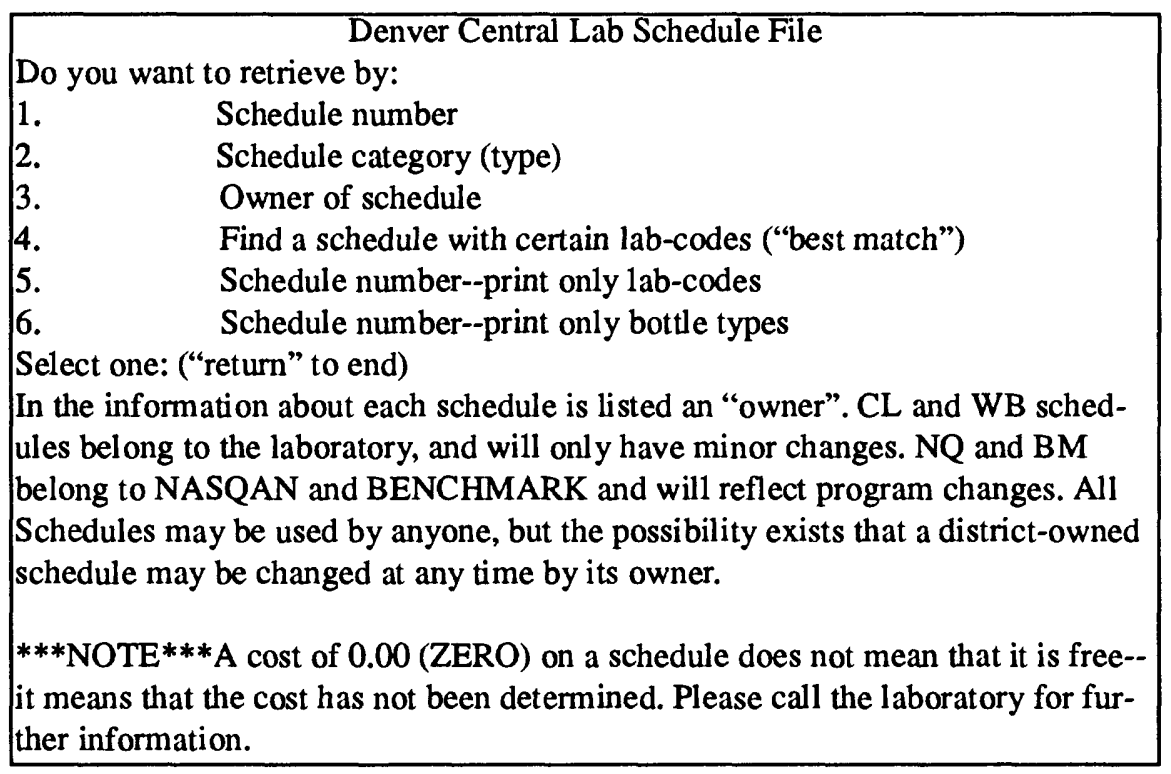

Suboption 1, by following subsequent menus, will retrieve information about a specific schedule such as owner, cost, and the constituent laboratory codes along with WATSTORE codes and the long name of the parameters. A second "page" will list bottle types and sample volumes.

Suboption 2 will list schedule types, useful in shortening searches performed with suboption 3.

Suboption 3 will list all schedules “owned” by any District, using that District's number.

Suboption 4 will search each (or all) schedule types for schedules that best match with the entered laboratory codes. This search is useful in determining if a schedule already exists with the desired parameters. Suboption 4 will list all schedules using a "score" based on the number of parameters matched, minus those not matched, and minus those in the schedule not requested. Many lower scoring schedules should be examined for applicability to the user's hydrologic problems, especially if there are "not requested" parameters. The Laboratory often includes parameters it needs for qual ity-control procedures ( $\mathrm{pH}$, specific conductance), or will include "free" parameters from procedures determining a number of constituents together, as for example, many organic analyses and some inorganic procedures such as metals by inductively coupled plasma.

Options 3 or 6 will allow several retrievals by showing the following screen:

\begin{tabular}{|l|}
\hline \multicolumn{1}{|c|}{ Central Lab Network File } \\
Do you want to retrieve by: \\
1. $\quad$ Unique number \\
2. Station-id \\
3. $\quad$ Unique number--Bottle types only \\
Select one: ("return" to end) \\
\hline
\end{tabular}




\section{GLOSSARY}

2SPE. The two-sigma percision estimate of a radiochemical measurement.

Accuracy. A measure of the degree of conformity of the values generated by a specific method or procedure with the true value. The concept of accuracy includes both bias (systematic error) and precision (random error) (Fishman and Friedman, 1989, p. 5).

Acid extraction. A procedure in which organic compounds are extracted from water samples with a solvent under acidic conditions.

Atomic absorption (AA). A technique of chemical analysis used mainly for determination of metallic elements. A water sample is aspirated into a laminar-flow flame which dries, desolvates, and atomizes the analyte(s). Light from a hollow cathode lamp of the element of interest is simultaneously shone through the flame, and its absorption by the analyte's vapor is measured. A comparison is made to absorption by standards treated in the same manner, and concentration is calculated.

Automated (auto.). Use of mechanical or computer modules to replace manual steps in chemical analysis procedures.

Automated-segmented flow colorimetry (ASF). A type of colorimetric analysis in which sample handling, reagent additions, mixing, and the colorimetric measurement itself all take place in small diameter tubes. The flow of fluids in the tubes is maintained by a peristaltic pump, and the reacting samples' volume is segmented by periodic introduction of air bubbles.

Base/neutral acid extraction (BNA). A procedure in which organic compounds are extracted from water samples with methylene chloride under basic conditions for later analysis by gas chromatography using a mass spectrometer as a detector.

Bias. Systematic error that is manifested as a consistent positive or negative deviation from the known or true value. It differs from random error which shows no such deviation.

Brine. Water that contains dissolved solids at an approximate concentration of $30,000 \mathrm{mg} / \mathrm{L}$ or more (American Society for Testing and Materials, 1993, p. 5).

Chain-of-custody (COC). A system in which the person-to-person custody of samples is documented from the time of collection until the samples are introduced as evidence in legal proceedings. The NWQL offers two levels of $\mathrm{COC}$ (with added charges commensurate with the level of security), covering the time of receipt of the samples at NWQL to the time of their disposal. These two levels are routine (from residence in a secured facility to full COC in which the samples are kept under lock and key) with a completely documented paper trail of all contacts within the NWQL.

Chelation extraction (chel.). A preparation and preconcentration method used prior to determination of a metal by atomic absorption (AA). A chemical complex of the metal to be determined is formed in the sample by reacting it with a chelating agent. The complex then is extracted into a smaller volume of organic solvent which then is determined by AA.

Chemical waste. Unusable by-products from many chemical and metal-processing operations that often contain toxic or hazardous materials that may become environmental contaminants if disposed of improperly.

Cold vapor atomic absorption (CVAA). A variation of atomic absorption in which the vapor of mercury is produced by chemical reduction and introduced into the photoabsorption area by a room-temperature carrier gas. 
Colorimetry. A technique of chemical analysis in which a sample of water is mixed with a reagent known to form a colored complex or reaction product with the analyte of interest. The light absorbed by the sample in a broad band near the wavelength of interest is measured and compared to standards, from which a concentration is calculated. Compared to spectrophotometry, detection limits are usually lower, but interferences are more pronounced.

Custom analysis (CA). An analysis involving constituents, sample matrices, or concentration levels for which the NWQL does not have established procedures or thoroughly tested prospective methods. Custom analyses normally would involve extensive methods adaptation, new method development, or purchase of new equipment of a type not in use at the NWQL. It will require, by its pioneering nature, a more intensive expenditure of personnel than routine service. Such work, if not disruptive of the routine operations of the NWQL, will be scheduled upon request to, and approval from, the NWQL Chief. Charges to the requester will be on a cost basis: $\$ 76$ per hour for FY 1994, plus costs of any purchased equipment and supplies. Analytical results will be reported by formal memorandum from the analyst, documenting the method, the result, and all available quality-assurance data, which characterize the precision and accuracy of the method, plus any available information on interferences.

Direct current plasma (DCP). A technique for chemical analysis of the metallic elements in which a plasma of argon gas is generated by a direct current discharge. A sample is aspirated into the plasma, and a characteristic atomic emission is observed. The emission spectrum is compared to standards treated in the same manner, and concentrations are calculated.

Dissolved (DIS). Pertains to the constituents in a representative water sample that pass through a 0.45 micrometer membrane filter or a 0.7 -micrometer glass fiber filter for organic analysis. This is a convenient operational definition used by Federal agencies that collect water data. Determinations of dissolved constituents are made on subsamples of the filtrate. In many cases, the words "dissolved" and "filtered" are used interchangeably.

Electrometric (elec.). Measurement of a physical or chemical property by the flow of electricity (for example, specific conductance).

Electrometric titration (ET). Potentiometric titration performed on a water sample to a fixed end point or to a stoichiometric equivalence point using a standard solution as a titrant.

Gas chromatography (GC). A method of chemical analysis used for organic compounds. The sample, or an extract of it, is injected into a heated port through which a carrier gas flows. The volatilized sample is swept into a long heated tube coated with a material (the stationary phase) that has varied affinities for the compounds of interest. These compounds are retarded in their passage by characteristic times. The exiting carrier gas then is analyzed by detectors sensitive to various physical/chemical properties (electron capture - ECD, flame ionization - FID, flame photometry-FPD, the presence of nitrogen or phosphorus - NPD), and responses generated by standard materials are compared.

Gas chromatography/mass spectrometry (GC/MS). A combination chemical analysis technique in which a mass spectrometer is used as a detector for a GC.

Graphite furnace atomic absorption (GFAA). A variation on atomic absorption (AA) in which a sample is injected into an electrically heated graphite tube placed in the area of photon absorption. Desolvation and vaporization occur separately under controlled conditions, and many interferences can be differentially vaporized. GFAA usually results in lower detection limits than AA.

Gravimetric analysis (GR). A method of chemical analysis in which the mass of a constituent is measured on a calibrated analytical balance. 
Hazardous analysis (HA). Any material requiring special handling that otherwise could damage the health or well-being of humans or the ecosystem. These materials include corrosives, flammables, and toxic agents such as drugs, chemicals and natural or synthetic products that are harmful, whose effects range from skin irritations to death. Arrangements with the NWQL must be made in advance for special laboratory handling techniques to be used.

High-performance liquid chromatography (HPLC). A technique of chemical analysis for determining organic compounds of too low a volatility or too high a thermal instability to use GC. The mobile phase is a mixture of solvents, sometimes dynamically varying throughout the analysis; the stationary phase resembles that of GC in having varying chemical affinities for the analytes; and detection is accomplished by a variety of techniques, including ultraviolet light absorption and fluorescence.

High solids (HS). A sample having dissolved solids greater than $250 \mathrm{mg} / \mathrm{L}$. Used in distinguishing between radiochemical methods of analysis.

Hydride generation (hyd). A chemical technique that forms gaseous hydrides of an element prior to its determination by AA. By forming a gaseous compound, the constituent can be removed from an aqueous matrix that might otherwise interfere with its determination.

Inductively coupled plasma (ICP). A method of analysis for the metallic elements in which an 8,000 to $10,000^{\circ} \mathrm{K}$ plasma of argon gas is electronically maintained by an induction coil. A sample is aspirated into the plasma, and characteristic atomic emissions are observed. The emission spectrum is compared to standards treated in the same manner, and concentrations are calculated. At the NWQL, up to 20 elements can be determined simultaneously by this method.

Ion-exchange chromatography (IC). A technique for chemical analysis of inorganic ions. With reference to GC, the mobile phase is aqueous, the stationary phase is a special ion-exchange resin of very low-ionic capacity, and the detection system is usually based on specific conductance.

Laboratory code (LC). A 1- to 4-digit code in the Services Catalog, always preceded by the letters "LC," which uniquely represents a parameter determined by a particular method of chemical analysis. For example, iron by AA and iron by ICP have different LCs.

Limited analysis (L). An analysis performed using an approved method but for which the Laboratory has only a limited capacity because of staffing level, space, equipment, or other considerations. As demand increases, the NWQL will try to budget for the necessary expansion, but meanwhile, time delays in reporting results can be expected if demand exceeds capacity. An arrangement for more than a few limited analyses requires considerable advanced planning for removal of the constraints. Project Chiefs requiring unusual numbers of limited analyses need to provide advance notice to the appropriate Program Chief of the NWQL as soon as the need becomes apparent, that is, during the early stages of project planning. In the case of large national programs, this lead time has been in the 2- to 4-year range and has included funding for expansion.

Low-ionic strength (LIS). Sample with a low dissolved-salt content (less than $100 \mu \mathrm{S} / \mathrm{cm}$ specific conductance). This is a criterion for accepting requests for specialized inorganic analyses. The LIS samples undergo specialized determinations developed specifically for these relatively pure samples.

Low-level analysis (LL). A type of inorganic analysis characterized by low levels of dissolved analytes. The criterion for this type of analysis varies for each specific constituent. Samples for LL determinations need not be LIS samples. 
Mass spectrometry (MS). A technique of chemical analysis usually determining trace elements. A sample or its extract is volatilized in a vacuum, and ions are produced. The analyte's ions usually break into smaller ions, and each mass is detected electronically. By the pattern of ions detected, the original (parent) compound's structure can be deduced. Alternately, the ions may come from the plasma of an ICP, and the analytically useful ones are atomic ions, which are determined without structural interpretation.

Method code (MC). A letter code associated with a parameter code that uniquely identifies the analytical method used to determine a constituent.

Methylene blue active substances (MBAS). A method of determining detergent-like compounds using colorimetry to detect their reaction product with methylene blue.

Minimum-reporting level (MRL). The smallest measured concentration of a constituent that may be reliably reported using a given analytical method.

Periphyton. The assemblage of microorganisms attached to and living upon submerged-solid surfaces.

Phytoplankton. The community of suspended or floating plants that drift passively with water currents.

Precision. The degree of similarity among independent measurements of the same quantity, without reference to the known or true value (V.R. Schneider, U.S. Geological Survey, written commun., 1990).

Priority analysis. The priority assigned to an analysis that will result in an expedited analysis. Arrangements for these priorities need to be made with the appropriate Program Chief(s) prior to submitting samples. Requests are submitted in writing, and confirmation from the Chief(s) will be in writing. If the requested turnaround time cannot be established, the Chief, NWQL, should be contacted to reconcile the request. If the priority work cannot be accommodated without disrupting the flow of work, a surcharge may be incurred.

Reanalysis. Reanalyzing a sample for the analyte in question. Reanalysis is requested when there is a discrepancy between the value in question and historical data, or when there is knowledge, such as site characteristics, that leads the project chief to believe the value is out of an expected range.

Recoverable bottom material. The amount of a given constituent that is in solution after a bottom-material sample has been extracted or digested by a method that results in dissolution of readily soluble substances. For inorganic determinations, digestions are performed in the original sample container to ensure digestion of material absorbed on the container walls. Complete dissolution of all bottom material is not commonly achieved by these treatments, and there is reason to believe that the determination represents less than the total amount (less than 95 percent) of the constituent sought in the sample. To ensure comparability of analytical data, equivalent digestion procedures (identical reagent strengths and volumes, identical digestion or extraction times, and identical temperatures) need to be used by all laboratories performing such analyses.

Regular analysis. An analysis that is performed routinely by the laboratory in which the concentration of the constituent of interest and the analytical interferences are within the limits specified in approved methods for that constituent, or need little additional work beyond simple dilution. Regular samples will be accepted by the NWQL without advance notice. Samples not meeting these criteria are classified as custom or special analyses.

Residue on evaporation (ROE). A gravimetric measure of volatile and nonvolatile substances in a water sample by evaporation and drying to $105^{\circ} \mathrm{C}$, then weighing the remaining substances. ROE also may be performed at $180^{\circ}$ and $550^{\circ} \mathrm{C}$.

Sample designation. Symbols that specify the type of container and pre-treatment of the sample. These symbols must be marked on the sample container. 
Schedule number (SC). A one- to four-digit number which denotes a group of laboratory codes determined as a unit. Schedules may be formulated by the NWQL, by a national program, or by a District . Schedules not formulated by the NWQL need to be submitted to the NWQL first to be checked for chemical rationality, length of parameter list, and price. Schedules must contain parameters of one type only (for example, "WO" organic may not be mixed with "WI" inorganic). Any non-NWQL owner may change or delete their schedule contents at any time.

Special analysis. An analysis not routinely performed in the NWQL. These analyses usually do not involve major efforts of development as do custom analyses, but may involve modifications for special matrix or concentration effects. However, they may involve timing or pricing considerations, such as request for a priority status, or special pricing for unusually large batches of samples. Requests for special analyses follow the form for custom analyses: a written request to, and reply from, the Chief, NWQL, an agreement on price and turnaround time, and an agreement on technical approach to the problem.

Spectrophotometry. A technique of chemical analysis in which a sample of water is mixed with a reagent known to form a colored complex or reaction product with the parameter of interest. The light absorbed by the sample at a specific wavelength is measured and compared to standards. Compared to colorimetry, this technique is less prone to interferences from other reaction products or from colored constituents in the sample.

Spike. An addition of a known quantity of one or more compounds of interest to the sample prior to analysis. This analysis yields data on the results that can be expected from a suite of similar samples (accuracy) when used with a synthetic matrix, and on the influence of matrix effects (recovery data) when used with the sample's matrix.

Surrogate. A compound similar in physical and chemical properties to the analytes of interest, which is added to the sample upon receipt in the laboratory (or ideally, at the time of field sampling). A surrogate is not used as an internal standard for quantitative measurement purposes. Surrogates may be added to every sample to provide quality control by monitoring for matrix effects and gross sample-processing errors. They should not occur naturally or be present in polluted samples. Also called "surrogate spike."

Suspended, recoverable. Pertains to the constituents extracted from the suspended sediment that is retained on a filter. Complete extraction generally is not achieved, and thus the determination represents something less than the "total" amount (that is, less than 95 percent) of the constituent present in the suspended phase of the sample. To achieve comparability of analytical data, laboratories performing such analyses would have to use equivalent-extraction procedures because different extraction procedures are likely to produce different analytical results. Determination of "suspended, recoverable" constituents is made either by analyzing portions of the material collected on the filter or, more commonly, by computing the difference between (1) dissolved and (2) total recoverable concentrations of the constituent.

Suspended, total. Pertains to the constituents of the suspended materials that are retained on a filter. This term is used only when the analytical procedure ensures measurement of at least 95 percent of the constituent determined. Knowledge of the expected form of the constituent in the sample, as well as of the analytical methodology used, is required to determine when the results should be reported as "suspended, total." Determinations of "suspended, total" constituents are made either by analyzing portions of the material collected on the filter or, more commonly, by computing the difference between (1) dissolved and (2) total concentrations of the constituent.

Titrimetry. A technique of chemical analysis in which an accurately measured volume of solution of known concentration reacts with an exact quantity of the substance being determined. 
Total. Pertains to the constituents in a representative water-suspended-sediment sample. This term is used only when the analytical procedure ensures measurement of at least 95 percent of the constituent present in both the dissolved and suspended phases of the sample. Knowledge of the expected form of the constituent in the sample, as well as of the analytical methodology used, is required to judge when the results should be reported as "total." (Note that the word "total" does double duty here, indicating that the sample consists of a water-suspended-sediment mixture and that the analytical method determines all of the constituents in the sample.)

Total in bottom material. Pertains to constituents in a representative sample of bottom material. This term is used only when the analytical procedure ensures measurement of at least 95 percent of the constituent determined. Knowledge of the expected form of the constituent in the sample, as well as of the analytical methodology used, is required to judge when the results should be reported as "total in bottom material."

Verification. Analysts verify analytical results by reviewing paperwork or computer files that reflect the analysis that the sample in question is a part of. Any discrepancies are corrected and the customer notified of results.

Volatile on ignition (VOI). A gravimetric measure of the matter leaving a sample between the temperatures of 105 and $600^{\circ} \mathrm{C}$. Oxidation as well as true evaporation may be involved.

Volatile organic compounds (VOC). A compound having high-vapor pressure and low-water solubility. VOC are typically industrial solvents, constituents in petroleum-fuel products, or by-products produced by chlorination in water treatment.

Volume or weight needed. The volume or weight of sample necessary for analysis. When more than one determination is requested, it may be different from the sum of weights or volumes needed for each constituent because many NWQL techniques yield multiple determinations simultaneously. When sample amount is limited and a schedule is not used in the request for analytical services, the NWQL needs to be consulted about the actual quantity needed.

Whole water, recoverable (WWR). Pertains to the constituents in solution after a representative watersuspended-sediment sample is digested (usually using a dilute acid solution). Complete dissolution of particulate matter often is not achieved by the digestion treatment, and thus the determination represents something less than the "total" amount (that is, less than 95 percent) of the constituent present in the dissolved and suspended phases of the sample. For inorganic determinations, digestions are performed in the original sample container to ensure digestion of material absorbed on the container walls. To achieve comparability of analytical data, equivalent digestion procedures would be required of all laboratories performing such analyses because different digestion procedures are likely to produce different analytical results. 


\section{INDEX}

Schedules in numerical order

Sche-

dule

$\mathrm{No}$

Herbicides: Chlorophenoxy-acid, with dicamba and picloram, WWR, by GC/ECD .......................49

Herbicides: Chlorophenoxy-acid, with dicamba and picloram, BTM, by GC/ECD ........................45

Major cations plus iron and manganese, inductively coupled .........................................................39

plasma, abbreviated list. Specific conductance needs to be less than $6,000 \mu \mathrm{S} / \mathrm{cm}$

Gross alpha and gross beta, suspended.

Gross alpha and gross beta, field filtered; radium and uranium, field filtered ................................6 67

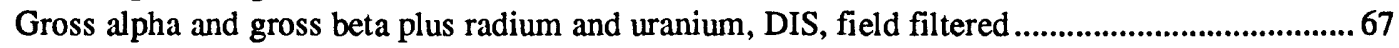

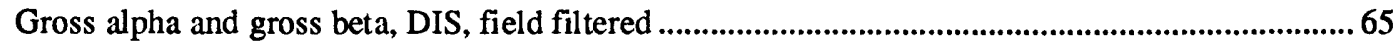

Gross alpha and gross beta, field filtered, to be used if solids are greater than $250 \mathrm{mg} / \mathrm{L}$

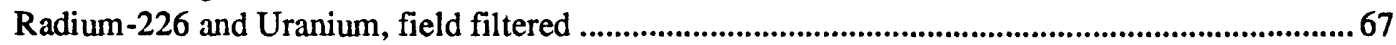

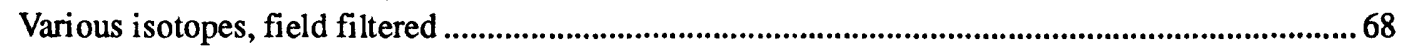

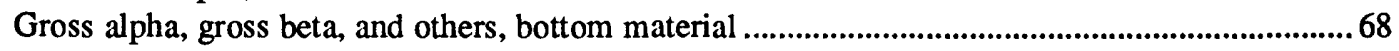

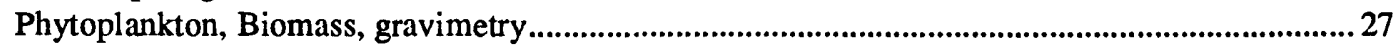

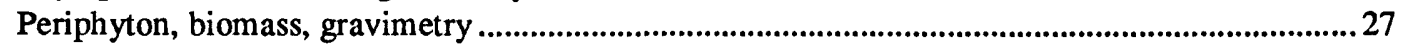

Sulfur-34/Sulfur-3636, rock; sulfate, disulide, monosulfide .....................................................70

Major cations plus trace metals, inductively coupled plasma;

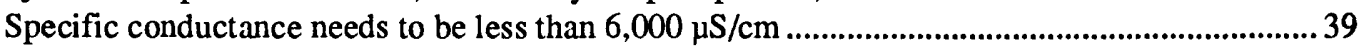

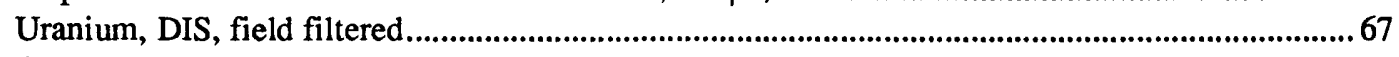

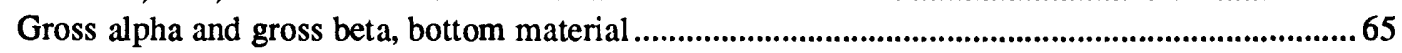

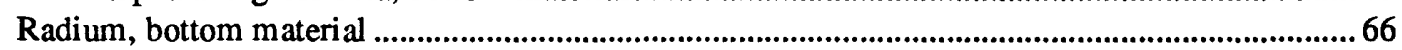

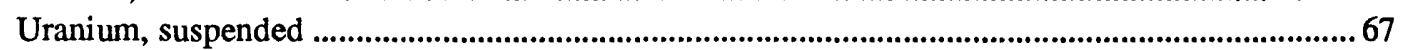

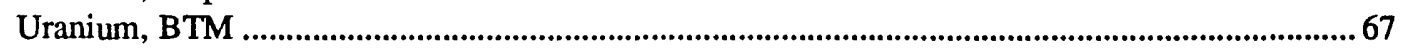

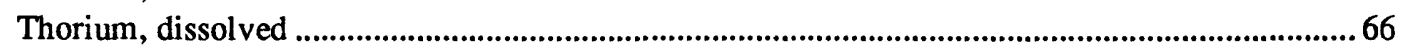

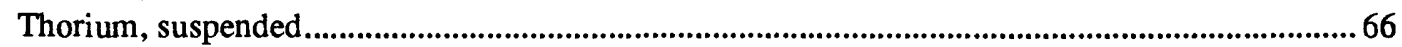

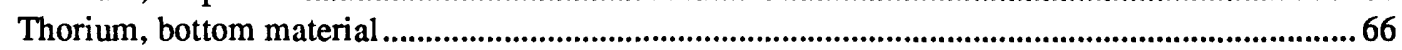

Oxygen-18/Oxygen-16 ratio; deuterium/protium, aqueous .............................................................. 70

Herbicides: Chlorophenoxy-acid, DIS, by GC/ECD ...............................................................49

Herbicides: Chlorophenoxy-acid, WWR, by GC/ECD ..................................................................... 49

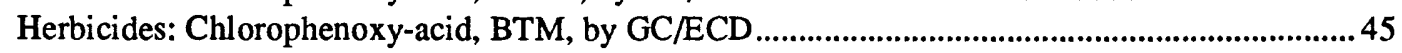

Volatile organic compounds: Soil fumigants, ECB \& DBCP,

WWR, by hexane micro-extraction, GC/ECD ....................................................................... 49

Volatile organic compounds, SDWA regulated

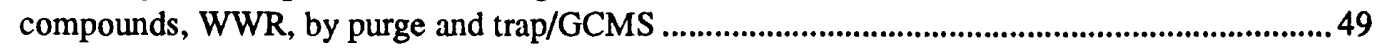

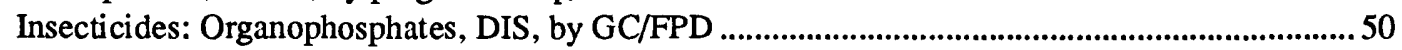

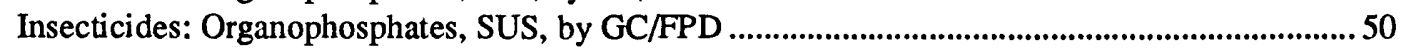

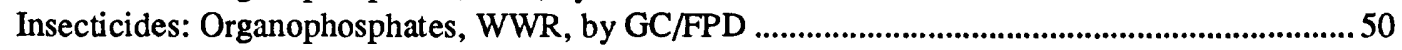

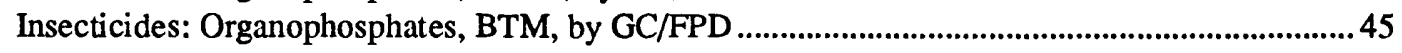

Insecticides: Organochlorine, with gross PCBs and gross PCNs, DIS, GC/ECD ............................50

Insecticides: Organochlorine, with gross PCBs and gross PCNs, WWR, by GC/ECD ...................51

Insecticides: Organochlorine, with gross PCBs and gross PCNs, BTM, by GC/ECD .................... 45

Insecticides: Organochlorine, with gross PCBs and gross PCNs, and

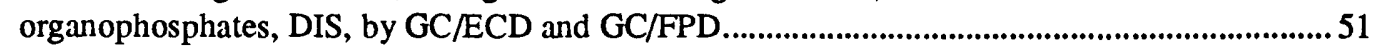

Insecticides: Organochlorine, with gross PCBs and gross PCNs, and organophosphates, WWR, by GC/ECD and GC/FPD. 


\section{INDEX \\ Schedules in numerical order--Continued}

Sche-

dule

No Name phase, and method

1335 Insecticides: Organochlorine, with gross PCBs and gross PCNs, and organophosphates, BTM, GC/ECD and GC/FPD ......................................................................45

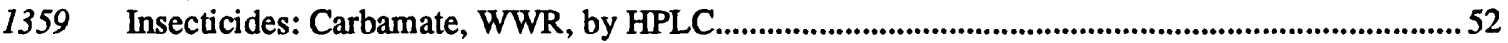

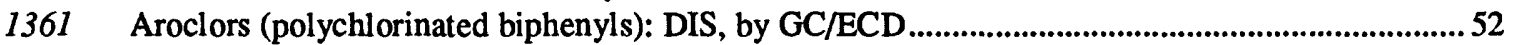

1364 Aroclors (polychlorinated biphenyls): WWR, by GC/ECD .....................................................52

1378 Volatile organic compounds: BTEX (benzene, toluene, and xylenes), WWR, by purge and trap GC/MS .................................................................................52

1379 Herbicides: Triazines, DIS, by GC/MS ..............................................................................................5 53

1380 Volatile organic compounds, SDWA regulated and unregulated compounds. Also covers NPDES. WWR, by purge and trap GC/MS .................................................................... 44

1381 Semivolatiles scan: WWR, methylene chloride extractable .............................................................5 53 by GC/FID. Data and chromatogram are mailed to requester. No specific compounds are identified. ..............................................................................................5

1382 Semivolatiles scan: BTM, methylene chloride extractable compounds, by GC/FID. Data and chromatogram are mailed to requester. No specific compounds are identified. ............46

1383 Semivolatiles: WWR; acidic, basic and neutral methylene chloride extractable

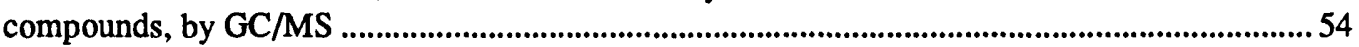

1384 Semivolatiles: BTM, methylene chl oride extractable compounds, by GC/MS.............................. 46

1385 Semivolatiles listed plus a tentative identification of most other compounds possible: WWR; acidic, basic and neutral methylene chloride extractable compounds, by GC/MS.

Letter report sent to requester.

Semivolatiles listed plus a tentative identification of all other compounds possible: BTM; acidic, basic and neutral methylene chloride extractable compounds, by GC/MS. Letter report sent to requester.

Volatile organic compounds: WWR, by purge and trap GC/MS (Same as schedule 1380, except for higher reporting levels.).

Volatiles organic compounds listed plus a tentative identification of all other compounds possible: WWR, by purge and trap GC/MS. Letter report sent to requester..............57

Semivolatiles: WWR; acidic methylene chloride extractable compounds, by GC/MS

Semivolatiles: WWR, basic and neutral methylene chloride extractable compounds, by GCMS

Insecticides: Organochl orine, with gross PCBs and gross PCNs, LL, WWR, by GC/ECD ...........58

Insecticides: Organochlorine, with gross PCBs and gross PCNs, and organophosphates,

LL, WWR, by GC/ECD and GC/FPD.

1401 Acrolein acrylonitrile and 2-chlorethylvinylether total recoverable from whole water using purge and trap and GC/MS

1474 Herbicides and insecticides: Combined schedule of Chlorophenoxy-acid, Organochlorine, with gross PCBs and gross PCNs, and organophosphates, WWR, by GC/ECD and GC/FPD.... 59

Periphyton, chlorophyll, chromatography and fluorometry

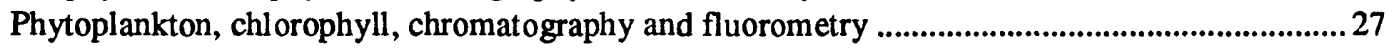

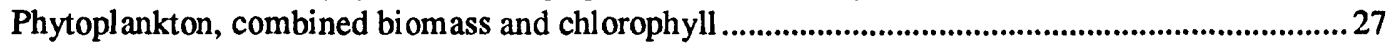

Insecticides: Organochlorine, with specific Aroclors, NPDES, WWR, by GC/ECD ......................59

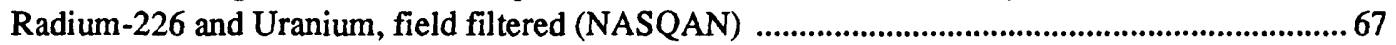

Periphyton, Combined biomass and chlorophyll. 
INDEX

Schedules in numerical order--Continued

Sche-

dule

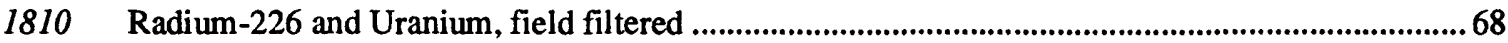

2001 Pesticides in filtered water, NWQL extracted on C-18 SPE cartridge analyzed by GC/MS ..........60

2010 Pesticides in filtered water, field extracted on C-18 SPE cartridge analyzed by GC/MS ................60

2050 Pesticides in filtered water, NWQL extracted on Carbopak B SPE cartridge

analyzed by HPLC ................................................................................................................................ 61

2051 Pesticides in filtered water field extracted on Carbopak B SPE cartridge, analyzed by HPLC ...... 61

2075 Gross organics in surface water for NAWQA ...................................................................................6. 62

2085 Gross organics in ground water for NAWQA.....................................................................................62

2090 VOC's at $0.2 \mu \mathrm{g} / \mathrm{L}$ by purge and trap GC/MS plus library search of nontarget constituents .......... 62

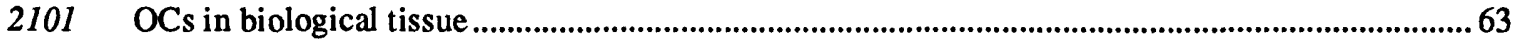

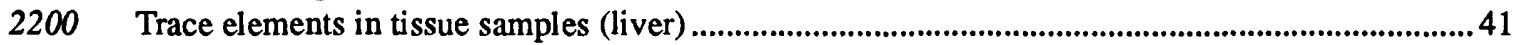

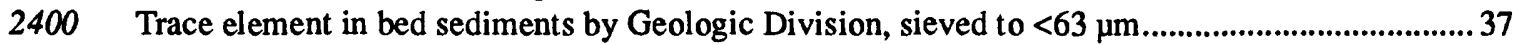

2501 Chlorinated organic compounds in bed sediments analyzed by GC ...............................................4 47

2502 Base-Neutral-Acid (BNA) semivolatile organic compounds in bed sediment by GC/MS ............. 48

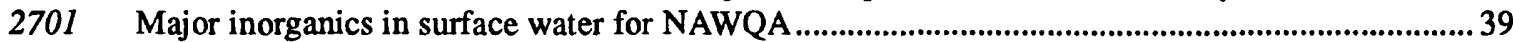

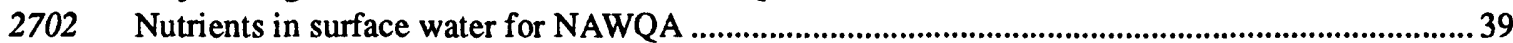

2703 Trace elements is ground water filtered through a 0.45 - $\mu \mathrm{m}$ filter for NAWQA................................40

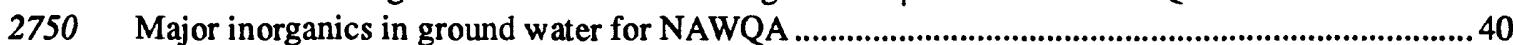

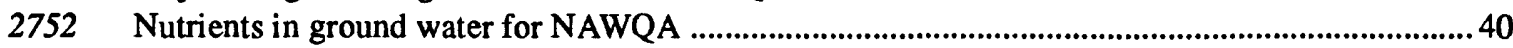


Index

Schedules in alphabetical order

Sche-

dule Page

No Name, phase, and method

Biological Schedules

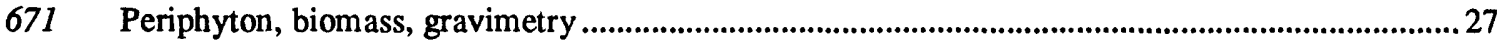

1507 Periphyton, chlorophyll, chromatography and fluorometry ...........................................................22

1708 Periphyton, Combined biomass and chlorophyll .............................................................................2

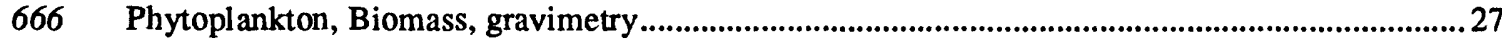

1508 Phytopl ankton, chlorophyll, chromatography and fluorometry ..................................................2 27

1509 Phytoplankton, combined biomass and chlorophyll ..................................................................2 27

Inorganic schedules

146 Major cations plus iron and manganese, inductively coupled plasma, abbreviated list

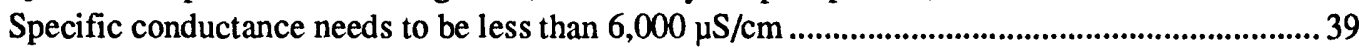

1043 Major cations plus trace metals, inductively coupled plasma.

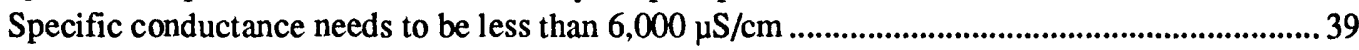

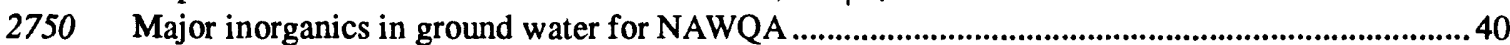

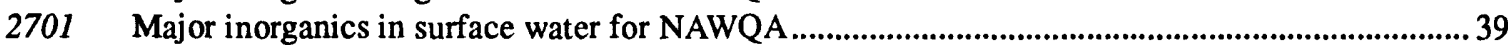

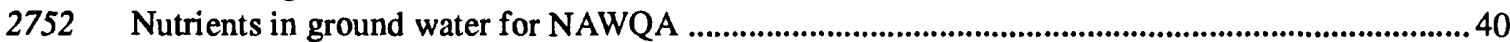

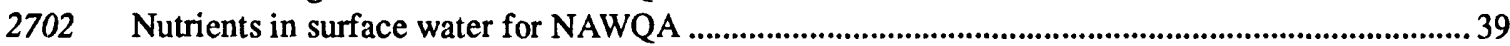

$2400 \quad$ Trace elements in bed sediments by Geologic Division, sieved to $<63 \mu \mathrm{m}$.................................... 37

2703 Trace elements in ground water filtered through a $0.45-\mu \mathrm{m}$ filter for NAWQA .............................40

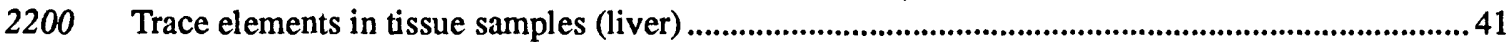

\section{Organic schedules}

1401 Acrolein acrylonitrile and 2-chlorethylvinylether total: WWR by GC/MS ....................................59

1364 Aroclors (polychlorinated biphenyls): WWR, by GC/ECD .........................................................52

1361 Aroclors (polychlorinated biphenyls): DIS, by GC/ECD ........................................................52

2502 Base-Neutral-Acid (BNA) semivolatile organic compounds in bed sediment by GC/MS ............. 48

2501 Chlorinated organic compounds in bed sediments analyzed by GC ...............................................47

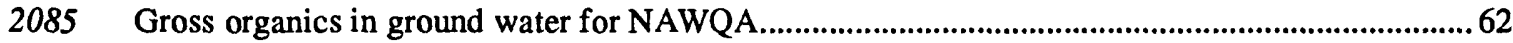

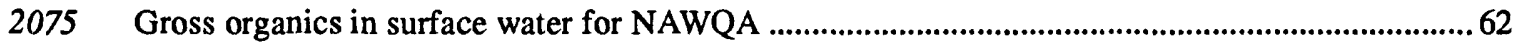

1474 Herbicides and insecticides: Combined schedule of Chlorophenoxy-acid, Organochlorine, with gross PCBs and gross PCNS, and organo phosphates,

WWR, by GC/ECD and GC/FPD

1305 Herbicides: Chlorophenoxy-acid, BTM, by GC/ECD ...................................................................45

1301 Herbicides: Chlorophenoxy-acid, DIS, by GC/ECD ......................................................................49

80 Herbicides: Chl orophenoxy-acid, with dicamba and picloram, BTM, by GC/ECD ......................45

79 Herbicides: Chlorophenoxy-acid, with dicamba and picloram, WWR, by GC/ECD .......................49

1304 Herbicides: Chlorophenoxy-acid, WWR, by GC/ECD ...................................................................49

1379 Herbicides: Triazines, DIS, by GC/MS ........................................................................................ 53

1389 Herbicides: Triazines, WWR, by GC/NPD ....................................................................................56

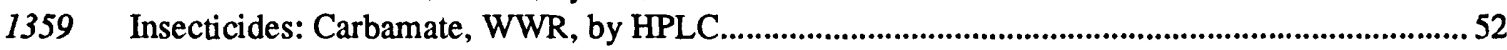

1321 Insecticides: Organochlorine, with gross PCBs and gross PCNs, DIS, GC/ECD .........................50

1335 Insecticides: Organochlorine, with gross PCBs and gross PCNs, and

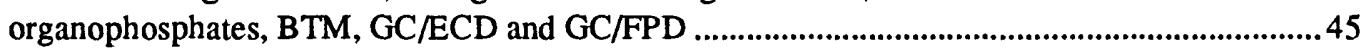

1331 Insecticides: Organochlorine, with gross PCBs and gross PCNs, and

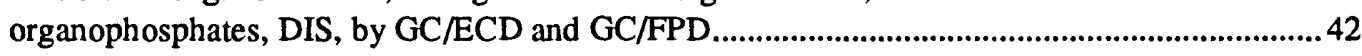

1399 Insecticides: Organochlorine, with gross PCBs and gross PCNs, and organophosphates, LL, WWR, by GC/ECD and GC/FPD ...................................................58 


\section{INDEX \\ Schedules in alphabetical order--Continued}

Sche-

dule

Page

No Name phase and method

No.

Organic schedules--continued

1334 Insecticides: Organochlorine, with gross PCBs and gross PCNs, and

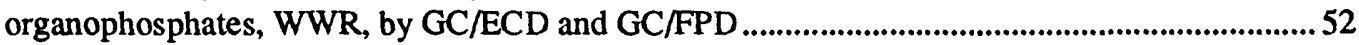

1325 Insecticides: Organochlorine, with gross PCBs and gross PCNs, BTM, by GC/ECD................... 45

1398 Insecticides: Organochlorine, with gross PCBs and gross PCNs LL, WWR, by GC/ECD ............ 58

1324 Insecticides: Organochlorine, with gross PCBs and gross PCNs, WWR, by GC/ECD .................. 51

1608 Insecticides: Organochlorine, with specific Aroclors, NPDES, WWR, by GC/ECD .....................55

1320 Insecticides: Organophosphates, BTM, by GC/FPD .......................................................................45

1316 Insecticides: Organophosphates, DIS, by GC/FPD .......................................................................50

1317 Insecticides: Organophosphates, SUS, by GC/FPD ….................................................................. 50

1319 Insecticides: Organophosphates, WWR, by GC/FPD ....................................................................5 50

2101 Organochlorine compounds (OCs) in biological tissue ..................................................................63 63

2010 Pesticides in filtered water field extraction C-18 SPE cartridge, analyzed by GC/MS...................60 60

2051 Pesticides in filtered water field extracted on Carbopak B SPE cartridge, analyzed by HPLC ...... 61

2001 Pesticides in filtered water NWQL extracted on C-18 SPE cartridge, analyzed by GC/MS ......... 60

2050 Pesticides in filtered water, NWQL extracted on Carbopak B SPE cartridge analyzed by HPLC.

1386 Semivolatiles listed plus a tentative identification of all other compounds possible:

BTM; acidic, basic and neutral methylene chloride extractable compounds, by GC/MS.

Letter report sent to requester.

1385 Semivolatiles listed plus a tentative identification of most other compounds possible:

WWR; acidic, basic and neutral methylene chloride extractable compounds, by GC/MS.

Letter report sent to requester.

1382 Semivolatiles scan: BTM, methylene chloride extractable compounds, by GC/FID.

Data and chromatogram are mailed to requester. No specific compounds are identified.

1381 Semivolatiles scan: WWR, methylene chloride extractable compounds, by GC/FID.

Data and chromatogram are mailed to requester. No specific compounds are identified. ............53

1384 Semivolatiles: BTM, methylene chloride extractable compounds, by GC/MS...............................46

1394 Semivolatiles: WWR, basic and neutral methylene chloride extractable

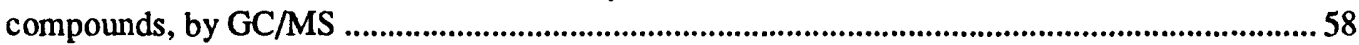

1393 Semivolatiles: WWR; acidic methylene chloride extractable compounds, by GC/MS .................. 57

1383 Semivolatiles: WWR; acidic, basic and neutral methylene chloride extractable 40 compounds, by GC/MS

1307 Volatile organic compounds, SDWA regulated compounds, WWR, by purge and trap GC/MS

1380 Volatile organic compounds, SDWA regulated and unregulated compounds.

Also covers NPDES. WWR, by purge and trap GC/MS .................................................................... 53

2090 Volatile organic compounds at $0.2 \mu \mathrm{g} / \mathrm{L}$ by purge and trap (GC/MS) plus

library search of non-target constituents

1306 Volatile organic compounds: Soil fumigants, EDB \& DBCP, WWR, by hexane microextraction, GC/ECD.

1378 Volatile organic compounds: BTX (benzene, toluene, and xylenes), WWR,

by purge and trap GC/MS

1387 Volatile organic compounds: Limited, by purge and trap (GC/MS) ............................................55

1390 Volatile organic compounds: WWR, by purge and trap GC/MS (Same as schedule 1380, except for higher reporting levels.)

1392 Volatiles organic compounds listed plus a tentative identification of all other compounds possible: WWR, by purge and trap GC/MS. Letter report sent to requester. 
INDEX

Schedules in alphabetical order--Continued

Sche-

dule

No. Name, phase and method

Radiochemical schedules

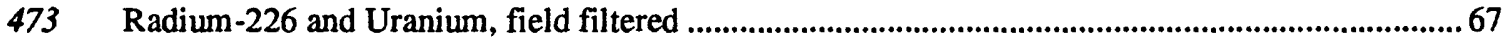

1703 Radium-226 and Uranium, field filtered (NASQAN) ...................................................................67

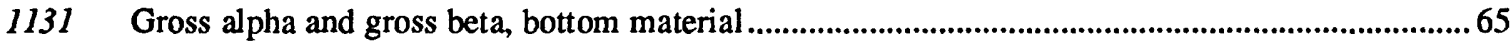

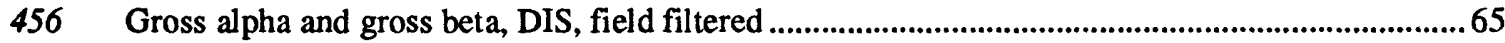

458 Gross al pha and gross beta, field filtered, to be used if solids are greater than $250 \mathrm{mg} / \mathrm{L} \ldots \ldots \ldots \ldots \ldots . . . .65$

214 Gross alpha and gross beta, field filtered; radium and uranium, field filtered ...............................6.67

308 Gross alpha and gross beta plus radium and uranium, DIS, field filtered .......................................67

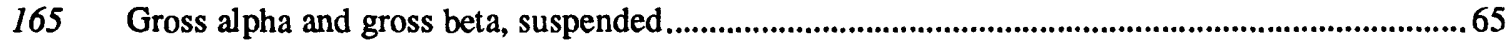

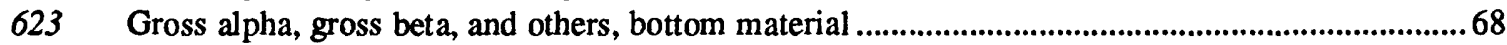

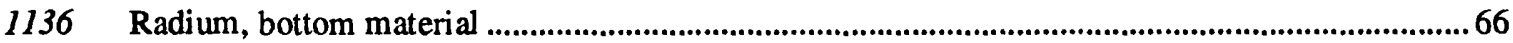

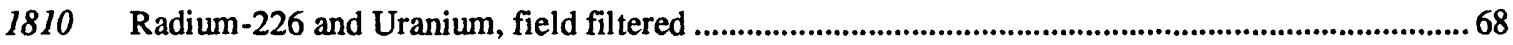

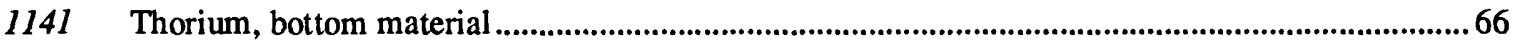

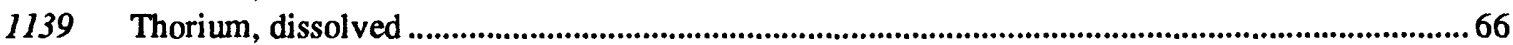

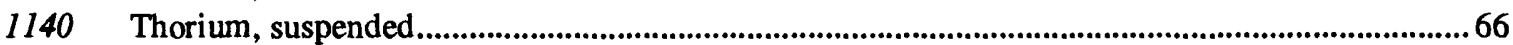

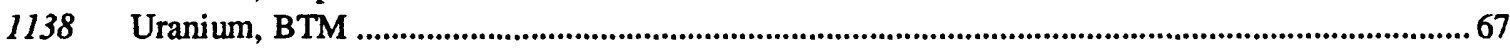

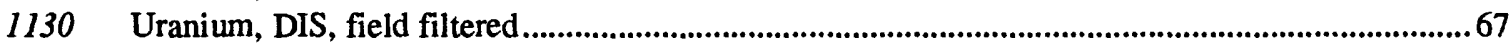

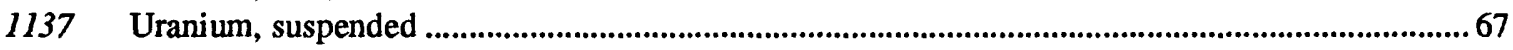

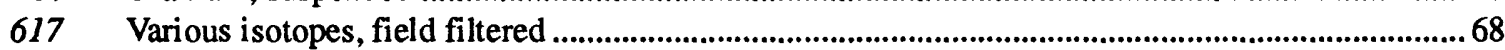

Stable isotopes

1142 Oxygen-18/Oxygen-16 ratio; deuterium/protium, aqueous............................................................ 70

921 Sulfur-34/Sulfur-32, rock; sulfate, disulfide, monosulfide ...........................................................70 


\begin{tabular}{|c|c|c|c|c|c|}
\hline Biological parameters & $\begin{array}{l}\text { Lab. } \\
\text { code }\end{array}$ & $\begin{array}{l}\text { Para- } \\
\text { meter } \\
\text { code }\end{array}$ & & $\begin{array}{l}\text { Sche- } \\
\text { dule }\end{array}$ & $\mathrm{Pg}$. \\
\hline Periphyton, biomass, ash weight & 611 & 572 & $\bar{A}$ & $\begin{array}{r}671 \\
1708\end{array}$ & $\begin{array}{l}27 \\
27\end{array}$ \\
\hline Periphyton, biomass, dry weight & 603 & 573 & $\mathbf{A}$ & $\begin{array}{r}671 \\
1708\end{array}$ & $\begin{array}{l}27 \\
27\end{array}$ \\
\hline Periphyton, chlorophyll a & 588 & 70957 & A & $\begin{array}{l}1507 \\
1708\end{array}$ & $\begin{array}{l}27 \\
27\end{array}$ \\
\hline Periphyton, chlorophyll b & 589 & 70958 & $\mathbf{A}$ & $\begin{array}{l}1507 \\
1708\end{array}$ & $\begin{array}{l}27 \\
27\end{array}$ \\
\hline Phytoplankton, biomass, ash weight & 621 & 81353 & $\mathbf{A}$ & $\begin{array}{r}666 \\
1509\end{array}$ & $\begin{array}{l}27 \\
27\end{array}$ \\
\hline Phytoplankton, biomass, dry weight & 620 & 81354 & A & $\begin{array}{r}666 \\
1509\end{array}$ & $\begin{array}{l}27 \\
27\end{array}$ \\
\hline Phytoplankton, chlorophyll a & 586 & 70953 & A & $\begin{array}{l}1508 \\
1509\end{array}$ & $\begin{array}{l}27 \\
27\end{array}$ \\
\hline Phytoplankton, chlorophyll b & 587 & 70954 & $\mathbf{A}$ & $\begin{array}{l}1508 \\
1509\end{array}$ & $\begin{array}{l}27 \\
27\end{array}$ \\
\hline Inorganic parameters & $\begin{array}{l}\text { Lab. } \\
\text { code }\end{array}$ & $\begin{array}{c}\text { Para- } \\
\text { meter } \\
\text { code }\end{array}$ & & $\begin{array}{l}\text { Sche- } \\
\text { dule }\end{array}$ & $\mathrm{Pg}$. \\
\hline Acidity as H, lab., LIS, W" & 1266 & $7 \overline{7825}$ & $\overline{\bar{B}}$ & $\overline{---}$ & $\overline{31}$ \\
\hline Acidity as $\mathrm{H}$, lab., WWR & 1 & 71825 & A & -- & 31 \\
\hline Alkalinity as $\mathrm{CaCO}_{3}$, lab. & 69 & 90095 & $\mathbf{A}$ & 2702 & 39 \\
\hline Alkalinity as $\mathrm{CaCO}_{3}$, lab., W" & 70 & 90410 & $\mathbf{A}$ & 2703 & $\begin{array}{l}31 \\
40\end{array}$ \\
\hline Alkalinity as $\mathrm{CaCO}_{3}$, lab., LIS, WWR & 1270 & 90410 & B & -- & 31 \\
\hline Alkalinity as $\mathrm{CaCO}_{3}$, lab., WWR, ET & 70 & 90410 & $\mathbf{A}$ & -- & 31 \\
\hline Aluminum, BTM, DCP & 1282 & 01108 & C & -- & 29 \\
\hline Aluminum, DIS, DCP & 1284 & 01106 & $\mathrm{E}$ & - & 31 \\
\hline Aluminum, DIS, ICP/MS & 1784 & 01106 & G & 2703 & 40 \\
\hline Aluminum, ICP & $\begin{array}{l}1736 \\
6000\end{array}$ & $\begin{array}{l}34790 \\
00000\end{array}$ & $\begin{array}{l}\mathbf{A} \\
\mathbf{A}\end{array}$ & $\begin{array}{l}2400 \\
2200\end{array}$ & $\begin{array}{l}37 \\
41\end{array}$ \\
\hline Aluminum, WWR, DCP & 1283 & 01105 & C & -- & 31 \\
\hline Antimony, BTM, AA, hydride & 534 & 01098 & $\mathbf{A}$ & - & 29 \\
\hline Antimony, DIS, AA, hydride & 77 & 01095 & $\mathbf{A}$ & -. & 31 \\
\hline Antimony, DIS, ICP/MS & 1785 & 01095 & $\mathbf{G}$ & 2703 & 40 \\
\hline Antimony, ICP/MS & 6018 & 00000 & B & 2200 & 41 \\
\hline Antimony, HA & 1776 & 34795 & $\mathbf{D}$ & 2400 & 37 \\
\hline Antimony, WWR, AA, hydride & 80 & 1097 & $\mathbf{A}$ & - & 31 \\
\hline Arsenic, BTM, AA, hydride & 597 & 1003 & $\mathbf{C}$ & & 29 \\
\hline Arsenic, DIS & 112 & 01000 & B & 2703 & 40 \\
\hline Arsenic, DIS, AA, hydride & 112 & 01000 & B & -- & 31 \\
\hline Arsenic, HA & 1775 & 34800 & D & 2400 & 37 \\
\hline Arsenic, ICP/MS & 6019 & 00000 & B & 2200 & 41 \\
\hline Arsenic, WWR, AA, hydride & 118 & 01002 & B & -- & 31 \\
\hline Arsenic, WWR, GFAA, USEPA & 1584 & 01002 & C & -- & 31 \\
\hline Barium, BTM, AA & 521 & 01008 & A & - & 29 \\
\hline Barium, DIS, AA & 7 & 01005 & B & -- & 31 \\
\hline Barium, DIS, ICP & 641 & 01005 & C & $\begin{array}{r}-- \\
1043\end{array}$ & $\begin{array}{l}31 \\
39\end{array}$ \\
\hline Barium, DIS, ICP/MS & 1786 & 01005 & G & 2703 & 40 \\
\hline
\end{tabular}

\begin{tabular}{|c|c|c|c|c|c|}
\hline Inorganic parameters--Continued & $\begin{array}{l}\text { Lab. } \\
\text { code }\end{array}$ & $\begin{array}{l}\text { Para- } \\
\text { meter } \\
\text { code }\end{array}$ & & $\begin{array}{l}\text { Sche- } \\
\text { dule }\end{array}$ & Pg. \\
\hline Barium, ICP & $\begin{array}{l}1745 \\
6001\end{array}$ & $\begin{array}{l}34850 \\
00000\end{array}$ & $\begin{array}{l}\bar{A} \\
A\end{array}$ & $\begin{array}{l}2400 \\
2200\end{array}$ & $\begin{array}{l}37 \\
41\end{array}$ \\
\hline Barium, WWR, AA & 234 & 01007 & $\mathbf{A}$ & -- & 31 \\
\hline Beryllium, BTM, AA & 522 & 01013 & $\mathbf{A}$ & -- & 29 \\
\hline Beryllium, DIS, AA & 170 & 01010 & $\mathbf{A}$ & $-\cdot$ & 31 \\
\hline Beryllium, DIS, ICP & 655 & 01010 & B & $\begin{array}{r}-- \\
1043\end{array}$ & $\begin{array}{l}31 \\
39\end{array}$ \\
\hline Beryllium, DIS, ICP/MS & 1787 & 01010 & G & 2703 & 40 \\
\hline Beryllium, ICP & 1746 & 34810 & $\mathbf{A}$ & 2400 & 37 \\
\hline Beryllium, ICP/MS & 6021 & 00000 & B & 2200 & 41 \\
\hline Beryllium, WWR, AA & 236 & 01012 & A & -- & 31 \\
\hline Bismuth, ICP & 1747 & 34816 & $\mathbf{A}$ & 2400 & 37 \\
\hline Boron, BTM, DCP & 1285 & 1023 & C & & 29 \\
\hline Boron, DIS, DCP & 1183 & 01020 & B & -- & 31 \\
\hline Boron, ICP & 6003 & 00000 & A & 2200 & 41 \\
\hline Boron, WWR, DCP & 1286 & 01022 & B & -- & 31 \\
\hline Bromide, DIS & 1246 & 71870 & E & 2750 & 40 \\
\hline Bromide, DIS, fluorescein, ASF & 1246 & 71870 & E & -- & 31 \\
\hline Bromide, LIS, DIS, IC & 1258 & 71870 & $\mathbf{F}$ & - & 31 \\
\hline Bromide,LIS,DIS,IC & 1258 & 1870 & $\mathbf{F}$ & & 31 \\
\hline Cadmium,BTM,AA & 502 & 01028 & B & -- & 29 \\
\hline Cadmium,DIS,AA & 126 & 01025 & A & -- & 31 \\
\hline Cadmium,DIS,GFAA & 1554 & 01025 & $\mathbf{F}$ & -- & 31 \\
\hline Cadmium,DIS,ICP & 673 & 01025 & $\begin{array}{l}\mathrm{D} \\
\mathrm{D}\end{array}$ & 1043 & $\begin{array}{l}31 \\
39\end{array}$ \\
\hline Cadmium, DIS, ICP/MS & 1788 & 01025 & G & 2703 & 40 \\
\hline Cadmium, DIS, GFAA & 1773 & 34825 & B & 2400 & 37 \\
\hline Cadmium, ICPCAMS & 6023 & 00000 & B & 2200 & 41 \\
\hline Cadmium,LL,DIS,GFAA & 1250 & 01025 & $\mathrm{E}$ & -- & 31 \\
\hline Cadmium,WWR,AA & 131 & 01027 & A & -- & 31 \\
\hline Cadmium,WWR,GFAA & 1555 & 01027 & $\mathbf{F}$ & -- & 31 \\
\hline Calcium,BTM,AA & 696 & 917 & A & - & 29 \\
\hline Calcium, DIS & 659 & 00915 & D & $\begin{array}{l}2701 \\
2750\end{array}$ & $\begin{array}{l}39 \\
40\end{array}$ \\
\hline Calcium,DIS,AA & 12 & 00915 & C & $-\cdot$ & 31 \\
\hline Calcium,DIS,ICP & 659 & 00915 & $\mathrm{D}$ & $\begin{array}{r}-- \\
146 \\
1043\end{array}$ & $\begin{array}{l}31 \\
39 \\
39\end{array}$ \\
\hline Calcium, ICP & 1737 & 43830 & A & 2400 & 37 \\
\hline Calcium,LIS,DIS,AA & 831 & 00915 & B & -- & 31 \\
\hline Calcium,LIS,DIS,ICP & 1273 & 00915 & $\mathrm{E}$ & -- & 31 \\
\hline Calcium,WWR,AA,USEPA & 324 & 00916 & A & -- & 31 \\
\hline Calcium,WWR,AA,USGS & 224 & 00916 & B & $\cdots$ & 31 \\
\hline Carbon, total & 1781 & 00000 & $\mathrm{~F}$ & 2400 & 37 \\
\hline Carbon, carbonate (inorganic) & 1782 & 00000 & $\mathbf{G}$ & 2400 & 37 \\
\hline Carbon, organic & 1783 & 00000 & $\mathbf{G}$ & 2400 & 37 \\
\hline Cerium, ICP & 1748 & 34835 & A & 2400 & 37 \\
\hline Chloride, DIS & 15 & 00940 & $\mathrm{E}$ & $\begin{array}{l}2701 \\
2750\end{array}$ & $\begin{array}{l}39 \\
40\end{array}$ \\
\hline Chloride,DIS,IC & 1571 & 00940 & $\mathbf{J}$ & -- & 31 \\
\hline Chloride,LIS,DIS,IC & 1259 & 00940 & I & -- & 31 \\
\hline Chromium,BTM,AA & 505 & 1029 & B & - & 29 \\
\hline
\end{tabular}




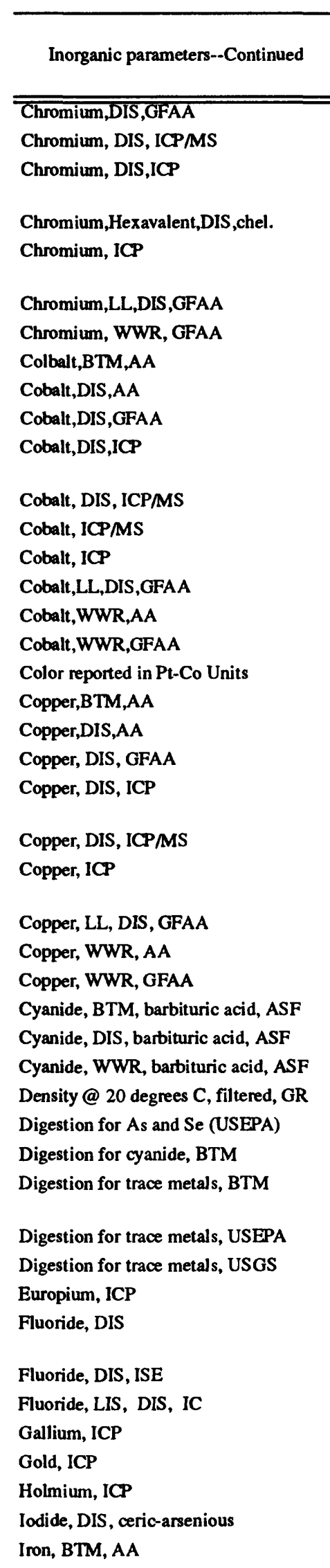

\begin{tabular}{lcc} 
Lab. & $\begin{array}{c}\text { Para- } \\
\text { meter } \\
\text { code }\end{array}$ & $\begin{array}{c}\text { Sche- } \\
\text { dule }\end{array}$ \\
\hline
\end{tabular}

$\begin{array}{llllll}1936 & 01030 & 1 & - & 32\end{array}$

$\begin{array}{lllll}1789 & 01030 & G & 2703 & 40\end{array}$

$\begin{array}{lllll}722 & 01030 & \text { E } & - & 32\end{array}$

$1043 \quad 39$

$\begin{array}{lllll}16 & 01032 & \text { A } & \text {-. } & 32\end{array}$

$\begin{array}{lllll}1750 & 34840 & \text { A } & 2400 & 37\end{array}$

600500000 A $2200 \quad 41$

$\begin{array}{lllll}1251 & 01030 & D & \ldots & 32\end{array}$

$\begin{array}{lllll}1937 & 01034 & \mathrm{E} & - & 32\end{array}$

$\begin{array}{lllll}506 & 01038 & \text { B } & \ldots & 29\end{array}$

$\begin{array}{lllll}148 & 01035 & \text { A } & - & 32\end{array}$

$\begin{array}{lllll}1556 & 01035 & \mathrm{~F} & \text {. } & 32\end{array}$

$64401035 \mathrm{C} \quad 32$

$\begin{array}{llll}1043 \quad 39 & \end{array}$

$\begin{array}{lllll}1790 & 1035 & G & 2703 & 40\end{array}$

$\begin{array}{lllll}6025 & 00000 & \text { B } & 2200 & 41\end{array}$

$\begin{array}{lllll}1749 & 34845 & \text { A } & 2400 & 37\end{array}$

$\begin{array}{lllll}1252 & 01035 & \mathrm{E} & -- & 32\end{array}$

$\begin{array}{lllll}149 & 01037 & \text { A } & \text {-. } & 32\end{array}$

$\begin{array}{lllll}1557 & 01037 & F & - & 32\end{array}$

$\begin{array}{lllll}20 & 00080 & \text { A } & - & 32\end{array}$

$\begin{array}{lllll}507 & 01043 & \text { B } & -- & 29\end{array}$

$\begin{array}{lllll}151 & 01040 & \text { A } & \text {.. } & 32\end{array}$

$\begin{array}{lllll}1558 & 01040 & F & \text { - } & 32\end{array}$

$\begin{array}{lllll}657 & 01040 & \mathrm{C} & \ldots & 32\end{array}$

$\begin{array}{lllll}1791 & 1040 & G & 2703 & 40\end{array}$

$\begin{array}{llllll}1751 & 34850 & \text { B } & 2400 & 37\end{array}$

$\begin{array}{lllll}6007 & 00000 & \text { A } & 2200 & 41\end{array}$

$\begin{array}{lllll}1253 & 01040 & \mathrm{E} & - & 32\end{array}$

$\begin{array}{lllll}156 & 01042 & \text { A } & - & 32\end{array}$

$\begin{array}{lllll}1559 & 01042 & \text { F } & \text { - } & 32\end{array}$

$\begin{array}{lllll}1235 & 00721 & \text { B } & - & 29\end{array}$

$\begin{array}{llllll}880 & 00723 & \text { A } & - & 32\end{array}$

$\begin{array}{llllll}23 & 00720 & \text { A } & -- & 32\end{array}$

$\begin{array}{lllll}24 & 71820 & \text { A } & - & 32\end{array}$

$\begin{array}{lllll}1586 & 00000 & \text { B } & - & 32\end{array}$

124299480 A 29

$\begin{array}{lll}647 & \text { LC } & \text { A }\end{array}$

$\begin{array}{rrrrr} & 0654 & & & \\ 124 \quad 99447 & \text { A } & - & 32\end{array}$

$\begin{array}{lllll}1735 & 99870 & \text { B } & \text { - } & 32\end{array}$

$\begin{array}{lllll}1752 & 34855 & \text { A } & 2400 & 37\end{array}$

$\begin{array}{lllll}1573 & 00950 & \text { E } & 2701 & 39\end{array}$

$\begin{array}{llll} & 2750 \quad 40\end{array}$

$\begin{array}{lllll}31 & 00950 & \text { B } & -- & 32\end{array}$

$\begin{array}{llllll}1260 & 00950 & \mathrm{D} & - & 32\end{array}$

$\begin{array}{lllll}1753 & 34860 & \text { A } & 2400 & 37\end{array}$

$\begin{array}{llllll}1744 & 34870 & \text { A } & 2400 & 37\end{array}$

$\begin{array}{lllll}1754 & 34875 & \text { A } & 2400 & 37\end{array}$

$\begin{array}{lllll}1202 & 71865 & \mathrm{D} & \ldots & 32\end{array}$

$\begin{array}{llllll}190 & 1170 & \text { B } & -- & 29\end{array}$

\begin{tabular}{|c|c|c|c|c|c|}
\hline Inorganic parameters--Continued & $\begin{array}{l}\text { Lab. } \\
\text { code }\end{array}$ & $\begin{array}{l}\text { Para- } \\
\text { meter } \\
\text { code }\end{array}$ & & $\begin{array}{l}\text { Sche- } \\
\text { dule }\end{array}$ & Pg. \\
\hline Iron, DIS & 645 & 01046 & $\overline{\mathrm{D}}$ & $\begin{array}{l}2701 \\
2750\end{array}$ & $\begin{array}{l}\overline{39} \\
40\end{array}$ \\
\hline Iron, DIS, AA & 172 & 01046 & $\mathbf{C}$ & -- & 32 \\
\hline Iron, DIS, ICP & 645 & 01046 & D & $\begin{array}{r}-- \\
146 \\
1043\end{array}$ & $\begin{array}{l}32 \\
39 \\
39\end{array}$ \\
\hline Iron, ICP & 6008 & $\begin{array}{l}34880 \\
00000\end{array}$ & $\mathbf{A}$ & $\begin{array}{l}2400 \\
2200\end{array}$ & $\begin{array}{l}37 \\
41\end{array}$ \\
\hline Iron, LIS, DIS, ICP & 1271 & 01046 & $\mathrm{E}$ & -- & 32 \\
\hline Iron, WWR, AA & 189 & 01045 & B & -- & 32 \\
\hline Lanthanum, ICP & 1755 & 34855 & B & 2400 & 37 \\
\hline Lead, BTM, AA & 510 & 01052 & B & -- & 29 \\
\hline Lead, DIS, AA & 191 & 01049 & $\mathbf{A}$ & -- & 32 \\
\hline Lead, DIS, GFAA & 1560 & 01049 & $\mathrm{~F}$ & -- & 32 \\
\hline Lead, DIS, ICP & 646 & 01049 & $\mathbf{C}$ & 1043 & $\begin{array}{l}32 \\
39\end{array}$ \\
\hline Lead, DIS, ICP/MS & 1792 & 1049 & $\mathbf{G}$ & 2703 & 40 \\
\hline Lead, ICP & 1762 & 34890 & $\mathbf{A}$ & 2400 & 37 \\
\hline Lead, ICP/MS & 6028 & 00000 & B & 2200 & 41 \\
\hline Lead, LL, DIS, GFAA & 1254 & 01049 & E & -- & 32 \\
\hline Lead, WWR, AA & 192 & 01051 & A & -- & 32 \\
\hline Lead, WWR, GFAA & 1561 & 01051 & $\mathbf{F}$ & -- & 32 \\
\hline Lithium, BTM, AA & 541 & 01133 & A & - & 29 \\
\hline Lithium, DIS, AA & 39 & 01130 & $\mathbf{A}$ & -- & 32 \\
\hline Lithium, DIS, ICP & 664 & 01130 & B & 1043 & $\begin{array}{l}33 \\
39\end{array}$ \\
\hline Lithium, ICP & 1756 & 34895 & $\mathbf{A}$ & 2400 & 37 \\
\hline Lithium, WWR, AA & 277 & 01132 & A & -- & 33 \\
\hline Magnesium, BTM, AA & 697 & 924 & A & - & 29 \\
\hline Magnesium, DIS & 663 & 00925 & C & $\begin{array}{l}2701 \\
2750\end{array}$ & $\begin{array}{l}39 \\
40\end{array}$ \\
\hline Magnesium, DIS, AA & 40 & 00925 & B & - & 33 \\
\hline Magnesium, DIS, ICP & 663 & 00925 & C & $\begin{array}{r}-- \\
146 \\
1043\end{array}$ & $\begin{array}{l}33 \\
39 \\
39\end{array}$ \\
\hline Magnesium, ICP & 1740 & 34900 & A & 2400 & 37 \\
\hline Magnesium, LIS, DIS, AA & 832 & 00925 & A & -- & 33 \\
\hline Magnesium, LIS, DIS, ICP & 1274 & 00925 & D & - & 33 \\
\hline Magnesium, WWR, USEPA, AA & 325 & 00927 & $\mathbf{A}$ & -- & 33 \\
\hline Magnesium, WWR, USGS, AA & 261 & 00927 & B & -- & 33 \\
\hline Manganese, BTM, AA & 512 & 1053 & A & -- & 29 \\
\hline Manganese, DIS & 648 & 01056 & C & $\begin{array}{l}2701 \\
2750\end{array}$ & $\begin{array}{l}39 \\
40\end{array}$ \\
\hline Manganese, DIS, AA & 42 & 01056 & $\mathbf{A}$ & - & 33 \\
\hline Manganese, DIS, ICP & 648 & 01056 & C & $\begin{array}{r}-- \\
146 \\
1043\end{array}$ & $\begin{array}{l}33 \\
39 \\
39\end{array}$ \\
\hline Manganese, DIS, ICP/MS & 1793 & 1056 & $\mathbf{G}$ & 2703 & 40 \\
\hline Manganese, ICP & $\begin{array}{l}1757 \\
6010\end{array}$ & $\begin{array}{l}34905 \\
00000\end{array}$ & $\begin{array}{l}\mathrm{A} \\
\mathrm{A}\end{array}$ & $\begin{array}{l}2400 \\
2200\end{array}$ & $\begin{array}{l}37 \\
41\end{array}$ \\
\hline Manganese, LIS, DIS, ICP & 1272 & 01056 & $\mathbf{E}$ & -- & 33 \\
\hline Manganese, LL, DIS, GFAA & 1255 & 01056 & D & -- & 33 \\
\hline
\end{tabular}


INDEX

Constituents in alphabetical order--Continued

\begin{tabular}{|c|c|c|c|c|c|}
\hline Inorganic parameters- & $\begin{array}{l}\text { Lab. } \\
\text { code }\end{array}$ & $\begin{array}{l}\text { Para- } \\
\text { meter } \\
\text { code }\end{array}$ & & $\begin{array}{l}\text { Sche- } \\
\text { dule }\end{array}$ & Pg. \\
\hline$\overline{\text { Manganese, WWR, } \overline{A A}}$ & $\overline{41}$ & 01055 & $\overline{\bar{A}}$ & $\overline{---}$ & $\overline{33}$ \\
\hline Mercury, BTM, CVAA & 511 & 71921 & $\mathbf{A}$ & -- & 29 \\
\hline Mercury, CVAA & $\begin{array}{l}1774 \\
6046\end{array}$ & $\begin{array}{l}34910 \\
00000\end{array}$ & $\begin{array}{l}\mathrm{C} \\
\mathrm{A}\end{array}$ & $\begin{array}{l}2400 \\
2200\end{array}$ & $\begin{array}{l}37 \\
41\end{array}$ \\
\hline Mercury, DIS, CVAA, auto. & 226 & 71890 & B & -- & 33 \\
\hline Mercury, WWR, CVAA & 227 & 71900 & B & -- & 33 \\
\hline Moisture content by weight & 904 & $\infty 0495$ & B & -- & 33 \\
\hline Molybdenum, BTM, AA & 523 & 01063 & A & -- & 29 \\
\hline Molybdenum, DIS, ICP & 649 & 01060 & $\mathbf{A}$ & - & 33 \\
\hline Molybdenum, DIS, chel., AA & 110 & 01060 & B & -- & 33 \\
\hline Molybdenum, DIS, ICP & 649 & 01060 & $\mathbf{A}$ & 1043 & $\begin{array}{l}33 \\
41\end{array}$ \\
\hline Molybdenum, DIS, ICPMS & 1794 & 01060 & G & 2703 & 40 \\
\hline Molybdenum, ICP & 1758 & 34915 & $\mathbf{A}$ & 2400 & 37 \\
\hline Molybdenum, ICP/MS & 6030 & 00000 & B & 2200 & 41 \\
\hline Molybdenum, WWR, AA & 265 & 01062 & $\mathbf{A}$ & $\because$ & 33 \\
\hline $\mathrm{N}$, Ammonia as $\mathrm{N}, \mathrm{BTM}$ & 524 & 00611 & A & -- & 29 \\
\hline $\mathrm{N}$, Ammonia as $\mathrm{N}$, DIS, colorimetric & 301 & 00608 & B & -- & 29 \\
\hline $\mathrm{N}$, Ammonia as $\mathrm{N}$, DIS, ASF & 301 & 00608 & B & -- & 39 \\
\hline $\mathrm{N}$, Ammonia as $\mathrm{N}, \mathrm{LL}$, DIS & 830 & 00608 & $\mathbf{A}$ & -- & 33 \\
\hline $\mathrm{N}$, Ammonia as N, LL, DIS & 1278 & 00608 & C & -- & 33 \\
\hline $\mathrm{N}$, Ammonia as N, LL, DIS, ASF & 830 & 00608 & $\mathbf{A}$ & & 37 \\
\hline $\mathrm{N}, \mathrm{NH} 3+$ organic $\mathrm{N}$ as $\mathrm{N}, \mathrm{DIS}$ & 1687 & 00623 & C & -- & 33 \\
\hline $\mathrm{N}, \mathrm{NH} 3+$ organic $\mathrm{N}$ as $\mathrm{N}, \mathrm{BTM}$ & 1211 & 00626 & C & -- & 29 \\
\hline $\mathrm{N}, \mathrm{NH} 3$ +organic $\mathrm{N}$ as $\mathrm{N}, \mathrm{WWR}$ & 1688 & 00625 & C & -- & 33 \\
\hline $\mathrm{N}$, Nitrate as $\mathrm{N}, \mathrm{LIS}, \mathrm{DIS}, \mathrm{IC}$ & 1261 & 00618 & D & - & 33 \\
\hline $\mathrm{N}$, Nitrite as $\mathrm{N}, \mathrm{DIS}$, diazotization & 160 & 00613 & B & -- & 33 \\
\hline $\mathrm{N}$, Nitrite as $N, \mathrm{LL}, \mathrm{DIS}, \mathrm{ASF}$ & 827 & 00613 & $\mathbf{A}$ & -- & 33 \\
\hline $\mathrm{N}$, Nitrite plus nitrate as N, BTM & 513 & 00633 & A & -- & 29 \\
\hline $\mathrm{N}$, Nitrite plus Nitrate as N, DIS & 228 & 00631 & B & -- & 33 \\
\hline $\mathrm{N}$, Nitrite plus Nitrate as N, DIS & 1578 & 00631 & $\mathrm{C}$ & -- & 33 \\
\hline $\mathrm{N}$, Nitrite plus Nitrate as $\mathrm{N}, \mathrm{LL}, \mathrm{DIS}$ & 826 & 00631 & A & -- & 33 \\
\hline $\mathrm{N}$, Total nitrogen as N, DIS, Antek & 1570 & 00602 & B & -- & 33 \\
\hline Neodymium, ICP & 1760 & 34920 & A & 2400 & 37 \\
\hline Nickel, BTM, AA & 519 & 01068 & B & -- & 29 \\
\hline Nickel, DIS, AA & 197 & 01065 & $\mathbf{A}$ & -- & 33 \\
\hline Nickel, DIS, GFAA & 1562 & 01065 & $\mathrm{~F}$ & -- & 33 \\
\hline Nickel, DIS, ICP & 721 & 01065 & $\mathrm{E}$ & 1043 & $\begin{array}{l}34 \\
39\end{array}$ \\
\hline Nickel, DIS, ICP/MS & 1795 & 1065 & G & 2703 & 40 \\
\hline Nickel, ICP & 1761 & 34925 & A & 2400 & 37 \\
\hline Nickel, ICPMS & 6031 & 00000 & B & 2200 & 41 \\
\hline Nickel, LL, DIS, GFAA & 1256 & 01065 & D & -- & 34 \\
\hline Nickel, WWR, GFAA & 1563 & 01067 & $\mathrm{~F}$ & -- & 34 \\
\hline Nickel, WWR, AA & 198 & 01067 & $\mathbf{A}$ & -- & 34 \\
\hline Niobium, ICP & 1759 & 34930 & $\mathbf{A}$ & 2400 & 37 \\
\hline Nitrogen, Ammonia, as N, DIS & 301 & 00608 & B & $\begin{array}{l}2702 \\
2752\end{array}$ & $\begin{array}{l}39 \\
40\end{array}$ \\
\hline Nitrogen, Ammonia+Organic, & 1687 & 00623 & C & $\begin{array}{l}2702 \\
2752\end{array}$ & $\begin{array}{l}39 \\
40\end{array}$ \\
\hline
\end{tabular}

\begin{tabular}{|c|c|c|c|c|c|}
\hline Inorganic parameters--Continued & $\begin{array}{l}\text { Lab. } \\
\text { code }\end{array}$ & $\begin{array}{l}\text { Para- } \\
\text { meter } \\
\text { code }\end{array}$ & & $\begin{array}{l}\text { Sche- } \\
\text { dule }\end{array}$ & Pg. \\
\hline Nitrogen,Ammonia+Organic,as N, Total & $\overline{1688}$ & $\overline{00625}$ & $\overline{\mathrm{C}}$ & $\overline{2702}$ & $\overline{\overline{39}}$ \\
\hline Nitrogen, Nitrite, as N, DIS & 160 & 00613 & B & $\begin{array}{l}2702 \\
2752\end{array}$ & $\begin{array}{l}39 \\
40\end{array}$ \\
\hline Nitrogen, Nitrate+Nitrate,as N, DIS & 228 & 00631 & B & $\begin{array}{l}2702 \\
2752\end{array}$ & $\begin{array}{l}39 \\
40\end{array}$ \\
\hline Oxygen demand, chemical, BTM & 532 & 00339 & A & - & 29 \\
\hline Oxygen demand, chemical, water & 76 & 00340 & B & - & 34 \\
\hline $\mathrm{P}$, Phosphate as P, ortho, DIS, PPMB & 162 & 00671 & B & -- & 34 \\
\hline P, Phosphate as P, ortho, LIS, DIS, IC & 1262 & 00671 & 6 & - & 34 \\
\hline P, Phosphate as P, ortho, LL,DIS & 828 & 00671 & A & -- & 34 \\
\hline P, Phosphate as P, ortho, LL, DIS & 1277 & 00671 & D & - & 34 \\
\hline P, Phosphate, ortho plus hydratizable & 282 & 678 & A & -. & 34 \\
\hline P, Phosphate, ortho plus hydratizable & 279 & 00677 & A & -- & 34 \\
\hline P, Phosphorus as P, BTM, PPMB & 515 & 00668 & B & -- & 29 \\
\hline P, Phosphorus as P, DIS, PPMB, ASF & 1685 & 00666 & C & - & 34 \\
\hline P, Phosphorus as P, LL,DIS, PPMB & 829 & 00666 & A & -- & 34 \\
\hline P, Phosphorus as P, LL, WWR & 837 & 00665 & A & -- & 34 \\
\hline $\mathrm{P}, \mathrm{Phosphorus} \mathrm{as} \mathrm{P}, \mathrm{WWR}, \mathrm{PPMB}$ & 1686 & 00665 & $\mathrm{C}$ & -- & 34 \\
\hline $\mathrm{pH}$, lab. & 68 & 00403 & A & $\begin{array}{r}-- \\
2701 \\
2703 \\
2750\end{array}$ & $\begin{array}{l}34 \\
39 \\
40 \\
40\end{array}$ \\
\hline pH, lab., elec. & 68 & 00403 & A & $\begin{array}{r}1043 \\
146\end{array}$ & $\begin{array}{l}34 \\
39 \\
39\end{array}$ \\
\hline pH, lab., LIS, elec. & 1268 & 00403 & B & -- & 34 \\
\hline Phosphorus, as P, DIS & 1685 & 00666 & C & $\begin{array}{l}2702 \\
2752\end{array}$ & $\begin{array}{l}39 \\
40\end{array}$ \\
\hline Phosphorus, as P Total & 1686 & 00665 & $\mathrm{C}$ & 2702 & 39 \\
\hline Phosphorus, ICP & 1742 & 34935 & A & 2400 & 37 \\
\hline Phosphorus, Orthophosphate, as P, DIS & 162 & 00671 & B & $\begin{array}{l}2702 \\
2752\end{array}$ & $\begin{array}{l}39 \\
40\end{array}$ \\
\hline Potassium, BTM, AA & 698 & 00938 & A & -- & 29 \\
\hline Potassium, DIS & 54 & 00935 & A & $\begin{array}{r}-. \\
2701 \\
2750\end{array}$ & $\begin{array}{l}34 \\
39 \\
40\end{array}$ \\
\hline Potassium, DIS, AA & 54 & 00935 & B & -. & 34 \\
\hline Potassium, LIS, DIS, AA & 833 & 00935 & A & -- & 34 \\
\hline Potassium, ICP & 1739 & 34790 & A & 2400 & 37 \\
\hline Potassium, WWR, USEPA, AA & 327 & 00937 & A & - & 34 \\
\hline Potassium, WWR, USGS, AA & 321 & 00937 & B & -- & 34 \\
\hline Preparation for BTM & 1184 & $\begin{array}{r}\text { LC } \\
1184\end{array}$ & A & - & 29 \\
\hline ROE,DIS@180C & 27 & 70300 & A & $\begin{array}{l}2701 \\
2750\end{array}$ & $\begin{array}{l}39 \\
40\end{array}$ \\
\hline Scandium, ICP & 1763 & 34945 & A & 2400 & 37 \\
\hline Selenium, BTM, hydride & 517 & 01148 & A & -. & 30 \\
\hline Selenium, BTM, hydride, auto. & 517 & 01148 & A & - & 34 \\
\hline Selenium, DIS, hydride, auto. & 87 & 01145 & A & $\begin{array}{r}-- \\
2703\end{array}$ & $\begin{array}{l}34 \\
40\end{array}$ \\
\hline Selenium, DIS, hydride, auto. & 87 & 1145 & A & & 34 \\
\hline Selenium, HA & 1777 & 34950 & D & 2400 & 37 \\
\hline Selenium, ICP/MS & 6032 & 00000 & B & 2200 & 41 \\
\hline
\end{tabular}


INDEX

Constituents in alphabetical order--Continued

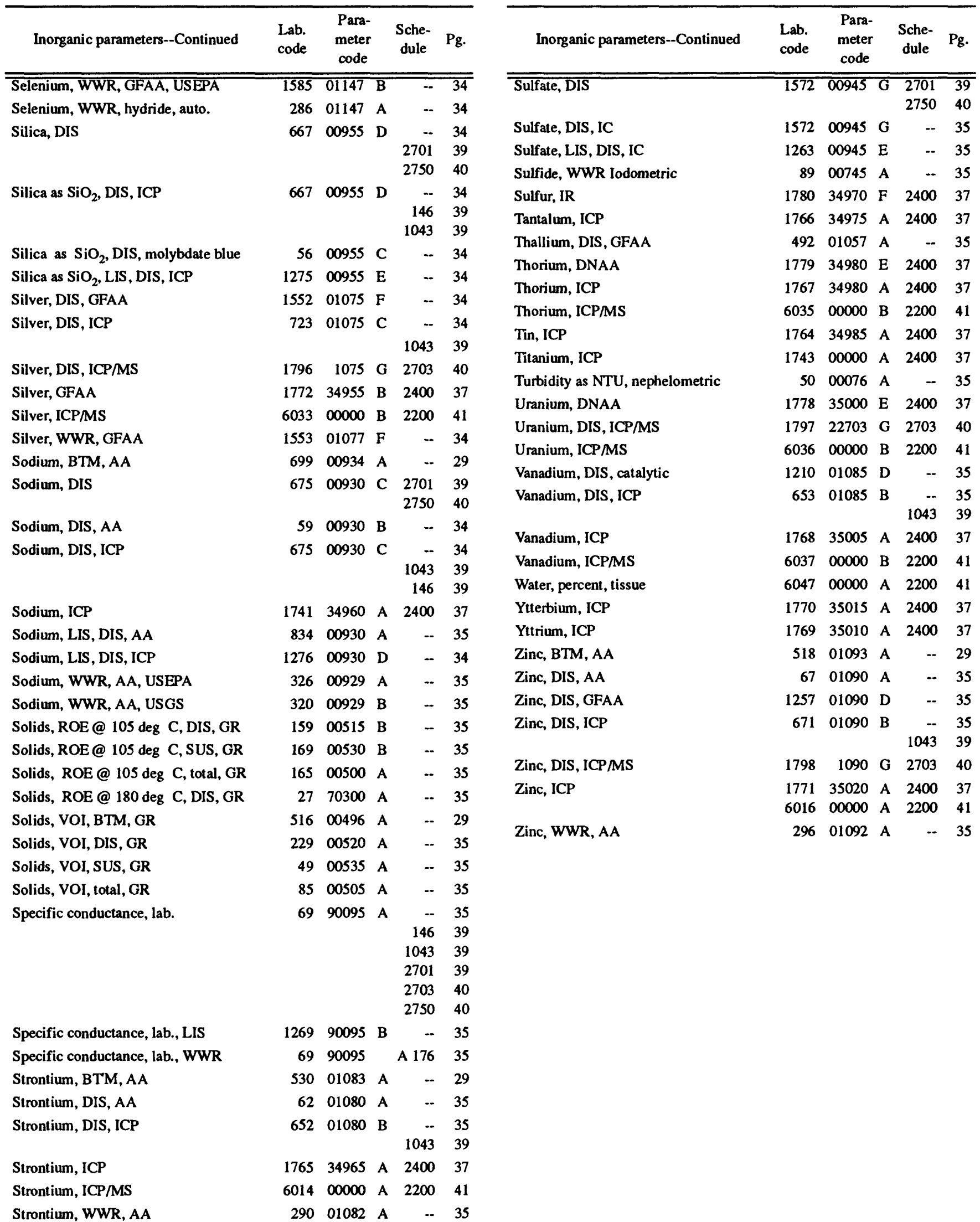




\begin{tabular}{l} 
Organic parameters \\
\hline 1,1,1,2-Tetrachloroethane \\
1,1,1,2-Tetrachloroethane \\
1,1,1-Trichloroethane \\
1,1,1-Trichloroethane \\
1,1,1-Trichloroethane \\
1,1,2,2-Tetrachloroethane \\
1,1,2,2-Tetrachloroethane \\
1,1,2,2-Tetrachloroethane \\
1,1,2-Trichloro-1,2,2-trifluoro
\end{tabular}

1,1,2-Trichloroethane

1,1,2-Trichloroethane

1,1-Dichloroethane

1,1-Dichloroethane

1,1-Dichloroethane

1,1-Dichloroethylene

1,1-Dichloroethylene

1,1-Dichloropropene

1,1-Dichloropropene

1,2,3-Trichlorobenzene

1,2,3-Trichlorobenzene

1,2,3-Trichloropropane

1,2,3-Trichloropropane

1,2,4-Trichlorobenzene

1,2,4-Trichlorobenzene

1,2,4-Trichlorobenzene

1,2,4-Trichlorobenzene

1,2,4-Trichlorobenzene

1,2,4-Trimethylbenzene

1,2,4-Trimethylbenzene

1,2,5,6-Dibenz[a,h]anthracene

1,2,5,6-Dibenz[a,h]anthracene

1,2-Dibromo-3-Chloropropane 1,2-Dibromo-3-Chloropropane

1,2-Dibromo-3-chloropropane (DBCP)

1,2-Dibromoethane

1,2-Dibromoethane

1,2-Dibromoethane (EDB)

1,2-Dichlorobenzene
Phase $\begin{array}{lll}\text { Lab. } & \text { Para- } & \text { Sche- } \text { Pg. } \\ \text { code meter } & \text { dule }\end{array}$

WWR 1693 T/562 A 1390 56 $\begin{array}{llllll}\text { WWR } & 1484 & 77562 & \text { B } & 1380 & 53\end{array}$ 139257

$\begin{array}{lllllll}\text { WWR } & 1309 & 34506 & \text { B } & 1307 & 49\end{array}$ WWR $1033 \quad 34506$ A $1390 \quad 56$ $\begin{array}{llllll}\text { WWR } & 1309 & 34506 & \text { B } & 1380 & 53\end{array}$ 139257

WWR $1030 \quad 34516$ A $1390 \quad 56$ $\begin{array}{llllll}\text { WWR } & 1306 & 34516 & \text { B } & 1380 & 53\end{array}$ $\begin{array}{llllll}\text { WWR } & 1306 & 34516 & \text { B } & 1392 & 57\end{array}$ $\begin{array}{llllll}\text { WWR } & 1681 \quad 77652 \text { A } 1307 \quad 49\end{array}$ $1380 \quad 53$ $1392 \quad 57$ 139056

WWR 103434511 A $1390 \quad 56$ WWR $1310 \quad 34511 \quad$ B $1380 \quad 53$ 139257

$\begin{array}{lllllll}\text { WWR } & 1297 & 34496 & \text { B } & 1307 & 49\end{array}$ $\begin{array}{llllll}\text { WWR } & 1021 & 34496 & \text { A } & 1390 & 56\end{array}$ $\begin{array}{llllll}\text { WWR } & 1297 & 34496 & \text { B } & 1380 & 53\end{array}$ 139257

$\begin{array}{llllll}\text { WWR } & 1023 & 34501 & \text { A } 1390 \quad 56\end{array}$ WWR 129934501 B 138053 139257 $1307 \quad 49$

$\begin{array}{lllllll}\text { WWR } & 1689 & 77168 \text { A } 1390 & 56\end{array}$ $\begin{array}{lllllll}\text { WWR } & 1478 \quad 77168 & \text { B } & 1380 & 53\end{array}$ 139257

$\begin{array}{llllll}\text { WWR } & 1680 & 77613 & \text { B } & 1390 & 56\end{array}$ WWR $1679 \quad 77613$ A 138053 139257

WWR 169577443 A 139056 $\begin{array}{llllll}\text { WWR } & 1483 \quad 77443 \text { B } 1380 & 53\end{array}$ 139257

$\begin{array}{lllllll}\text { BTM } 1166 \quad 34554 & \text { A } 1386 \quad 47\end{array}$ 138446

WWR $1111 \quad 34551$ A $1394 \quad 58$ WWR $1674 \quad 34551$ B $1390 \quad 56$ $\begin{array}{llllll}\text { WWR } & 1673 \quad 34551 & C & 1380 & 53\end{array}$ 139257

WWR $1111 \quad 34551$ A $1385 \quad 55$ $1383 \quad 54$

WWR 166677222 B 139056 $\begin{array}{llllll}\text { WWR } & 1665 & 77222 & \text { A } & 1380 & 53\end{array}$ \begin{tabular}{ll}
$1392 \quad 57$ \\
\hline
\end{tabular}

BTM 112934559 A $1386 \quad 47$ 138446

WWR 108334556 A $1394 \quad 58$ 138555 $1383 \quad 54$

$\begin{array}{llllll}\text { WWR } & 1349 \quad 82625 \text { A } 1390 & 56\end{array}$ $\begin{array}{lllllll}\text { WWR } & 1354 & 82625 & \text { B } & 1380 & 53\end{array}$ 139257

$\begin{array}{llllll}\text { WWR } & 1576 \quad 82625 \text { D } & 1306 & 49\end{array}$

$\begin{array}{llllll}\text { WWR } & 1319 & 77651 \mathrm{C} & 1390 & 56\end{array}$ $1380 \quad 53$

$\begin{array}{llllll}\text { WWR } & 1317 & 77651 & \text { B } & 1392 & 57\end{array}$ $\begin{array}{llllll}\text { WWR } & 1577 & 77651 & \text { D } & 1306 & 49\end{array}$ $\begin{array}{llllll}\text { BTM } \quad 1140 \quad 34539 & \text { A } & 1386 & 47\end{array}$

\begin{tabular}{|c|c|c|c|c|c|c|}
\hline Organic parameters--Continued & Phase & $\begin{array}{l}\text { Lab. } \\
\text { code }\end{array}$ & $\begin{array}{l}\text { Para- } \\
\text { meter }\end{array}$ & & $\begin{array}{c}\text { Sche- } \\
\text { dule }\end{array}$ & Pg. \\
\hline \multirow[t]{3}{*}{ 1,2-Dichorobenzene } & WWR & 7085 & 34536 & $\overline{\bar{A}}$ & 7385 & $\overline{55}$ \\
\hline & & & & & 1383 & 54 \\
\hline & & & & & 1394 & 58 \\
\hline \multirow[t]{2}{*}{ 1,2-Dichlorobenzene } & WWR & 1314 & 34536 & B & 1307 & 49 \\
\hline & & & & & 1380 & 53 \\
\hline \multirow[t]{2}{*}{ 1,2-Dichlorobenzene } & WWR & 1320 & 34536 & C & 1390 & 56 \\
\hline & & & & & 1392 & 57 \\
\hline 1,2-Dichloroethane & WWR & 1022 & 32103 & $\mathbf{A}$ & 1390 & 56 \\
\hline \multirow[t]{3}{*}{ 1,2-Dichloroethane } & WWR & 1298 & 32103 & B & 1307 & 49 \\
\hline & & & & & 1380 & 53 \\
\hline & & & & & 1392 & 57 \\
\hline 1,2-Dichloropropane & WWR & 1025 & 34541 & A & 1309 & 56 \\
\hline \multirow[t]{3}{*}{ 1,2-Dichloropropane } & WWR & 1301 & 34541 & B & 1307 & 49 \\
\hline & & & & & 1380 & 53 \\
\hline & & & & & 1392 & 57 \\
\hline 1,2-Diphenylhydrazine & WWR & 1697 & 82626 & $\mathbf{A}$ & 1383 & 54 \\
\hline \multirow[t]{2}{*}{ 1,3,5-Trimethylbenzene } & WWR & 1683 & 77226 & A & 1380 & 53 \\
\hline & & & & & 1392 & 57 \\
\hline 1,3,5-Trimethylbenzene & WWR & 1684 & 77226 & B & 1390 & 56 \\
\hline \multirow[t]{2}{*}{ 1,3-Dichlorobenzene } & BTM & 1141 & 34569 & $\mathbf{A}$ & 1386 & 47 \\
\hline & & & & & 1384 & 46 \\
\hline \multirow[t]{3}{*}{ 1,3-Dichlorobenzene } & WWR & 1086 & 34566 & A & 1394 & 58 \\
\hline & & & & & 1385 & 55 \\
\hline & & & & & 1383 & 54 \\
\hline \multirow[t]{3}{*}{ 1,3-Dichlorobenzene } & WWR & 1315 & 34566 & B & 1307 & 49 \\
\hline & & & & & 1380 & 53 \\
\hline & & & & & 1392 & 57 \\
\hline 1,3-Dichlorobenzene & WWR & 1321 & 34566 & C & 1390 & 56 \\
\hline 1,3-Dichloropropane & WWR & 1692 & 77173 & A & 1390 & 56 \\
\hline \multirow[t]{2}{*}{ 1,3-Dichloropropane } & WWR & 1480 & 77173 & B & 1380 & 53 \\
\hline & & & & & 1392 & 57 \\
\hline \multirow[t]{2}{*}{ 1,4-Dichlorobenzene } & BTM & 1142 & 34574 & A & 1386 & 47 \\
\hline & & & & & 1384 & 46 \\
\hline \multirow[t]{3}{*}{ 1,4-Dichlorobenzene } & WWR & 1087 & 34571 & A & 1385 & 55 \\
\hline & & & & & 1383 & 54 \\
\hline & & & & & 1394 & 57 \\
\hline \multirow[t]{3}{*}{ 1,4-Dichlorobenzene } & WWR & 1316 & 34571 & B & 1307 & 49 \\
\hline & & & & & 1380 & 53 \\
\hline & & & & & 1392 & 57 \\
\hline 1,4-Dichlorobenzene & WWR & 1322 & 34571 & C & 1390 & 56 \\
\hline 1,6-Dinitro-2-methylphenol & WWR & 1060 & 34657 & A & 1393 & 57 \\
\hline 1-Naphthol & WWR & 1351 & 77441 & A & 1359 & 52 \\
\hline \multirow[t]{2}{*}{ 1-Naphthol } & WWR & 5438 & -- & A & 2050 & 61 \\
\hline & & 5638 & - & A & 2051 & 61 \\
\hline 2,2'-biquinoline & BTM & 5285 & - & B & 2502 & 48 \\
\hline 2,2-Dichloropropane & WWR & 1691 & 77170 & A & 1390 & 56 \\
\hline \multirow[t]{2}{*}{ 2,2-Dichloropropane } & WWR & 1479 & 77170 & B & 1380 & 53 \\
\hline & & & & & 1392 & 57 \\
\hline $2,4,5-\mathrm{T}$ & DIS & 478 & 39742 & A & 1301 & 49 \\
\hline $2,4,5-\mathrm{T}$ & BTM & 3 & 39741 & A & 1305 & 45 \\
\hline & & & & & 80 & 45 \\
\hline $2,4,5-\mathrm{T}$ & WWR & 373 & 39740 & B & 1474 & 59 \\
\hline & & & & & 79 & 49 \\
\hline & & & & & 1304 & 49 \\
\hline $2,4,5-\mathrm{T}$ & WWR & 5409 & 39742 & B & 2050 & 61 \\
\hline & & 5609 & 39742 & B & 2051 & 61 \\
\hline 2,4,6-Trichlorophenol & BTM & 1054 & 34624 & A & 1386 & 47 \\
\hline & & & & & 1384 & 46 \\
\hline 2,4,6-Trichlorophenol & WWR & 1058 & 34621 & A & 1385 & 55 \\
\hline & & & & & 1393 & 57 \\
\hline & & & & & 1383 & 54 \\
\hline
\end{tabular}




\begin{tabular}{|c|c|c|c|c|c|c|c|c|c|c|c|c|c|}
\hline Organic parameters--Continued & Phase & $\begin{array}{l}\text { Lab. } \\
\text { code }\end{array}$ & $\begin{array}{l}\text { Para- } \\
\text { meter }\end{array}$ & & $\begin{array}{l}\text { Sche- } \\
\text { dule }\end{array}$ & Pg. & Organic parameters--Continued & Phase & $\begin{array}{l}\text { Lab. } \\
\text { code }\end{array}$ & $\begin{array}{l}\text { Para- } \\
\text { meter }\end{array}$ & & $\begin{array}{l}\text { Sche- } \\
\text { dule }\end{array}$ & Pg. \\
\hline$\overline{2,4-1}$ & DIS & $\overline{47 T}$ & 39732 & $\bar{A}$ & T301 & $\overline{49}$ & & & & & & 1393 & $\overline{5 T}$ \\
\hline \multirow[t]{2}{*}{$2,4-D$} & BTM & 375 & 39731 & $\mathbf{A}$ & 1305 & 45 & & & & & & 1383 & 54 \\
\hline & & & & & 80 & 45 & 2-Nitrophenol & BTM & 1050 & 34594 & A & 1386 & 47 \\
\hline \multirow[t]{3}{*}{ 2,4-D } & WWR & 372 & 39730 & B & 1474 & 59 & & & & & & 1384 & 46 \\
\hline & & & & & 79 & 49 & 2-Nitrophenol & WWR & 1062 & 34591 & A & 1385 & 55 \\
\hline & & & & & 1304 & 49 & & & & & & 1393 & 57 \\
\hline \multirow[t]{2}{*}{ 2,4-D } & WWR & 5408 & 39732 & B & 2050 & 61 & & & & & & 1383 & 54 \\
\hline & & 5608 & 39732 & B & 2051 & 61 & 3,3'-Dichlorobenzidine & WWR & 1088 & 34631 & A & 1385 & 55 \\
\hline \multirow[t]{2}{*}{ 2,4-DB } & WWR & 5407 & 38746 & $\mathbf{A}$ & 2050 & 61 & & & & & & 1383 & 54 \\
\hline & & 5607 & 38746 & $\mathbf{A}$ & 2051 & 61 & 3-Chloro-3-methylphenol & BTM & 1044 & 34455 & A & 1386 & 47 \\
\hline \multirow[t]{2}{*}{ 2,4-Dichlorophenol } & BTM & 1046 & 34604 & A & 1386 & 47 & 3-Hydrocarbofuran & WWR & 1353 & 82584 & A & 1359 & 52 \\
\hline & & & & & 1384 & 46 & 4,4'DDE & WWR & 1630 & 393201 & D & 1608 & 59 \\
\hline \multirow[t]{3}{*}{ 2,4-Dichlorophenol } & WWR & 1057 & 34601 & A & 1385 & 55 & 4,6-Dinitro-2-methylphenol & WWR & 1060 & 34657 & A & 1383 & 54 \\
\hline & & & & & 1393 & 57 & 4-Bromophenylphenyether & BTM & 1125 & 34639 & A & 1386 & 47 \\
\hline & & & & & 1383 & 54 & & & & & A & 1384 & 46 \\
\hline \multirow[t]{2}{*}{ 2,4-Dimethylphenol } & BTM & 1047 & 34609 & B & 1386 & 47 & 4-Bromophenylphenyether & WWR & 1079 & 34636 & A & 1394 & 58 \\
\hline & & & & & 1384 & 46 & & & & & A & 1385 & 55 \\
\hline \multirow[t]{3}{*}{ 2,4-Dimethylphenol } & WWR & 1059 & 34606 & $\mathbf{A}$ & 1385 & 55 & & & & & A & 1383 & 54 \\
\hline & & & & & 1393 & 57 & 4-Chloro-3-methylphenol & BTM & 1044 & 34455 & A & 1384 & 46 \\
\hline & & & & & 1383 & 54 & 4-Chloro-3-methylphenol & WWR & 1055 & 34452 & $\mathbf{A}$ & 1393 & 57 \\
\hline \multirow[t]{2}{*}{ 2,4-Dinitrophenol } & BTM & 1049 & 34619 & A & 1386 & 47 & & & & & & 1383 & 54 \\
\hline & & & & & 1384 & 46 & 4-Chlorophenylphenyether & BTM & 1127 & 34644 & A & 1386 & 47 \\
\hline \multirow[t]{3}{*}{ 2,4-Dinitrophenol } & WWR & 1061 & 34616 & $\mathbf{A}$ & 1385 & 55 & & & & & & 1384 & 46 \\
\hline & & & & & 1393 & 57 & 4-Chlorophenylphenyether & WWR & 1081 & 34641 & B & 1394 & 58 \\
\hline & & & & & 1383 & 54 & & & & & & 1385 & 55 \\
\hline \multirow[t]{2}{*}{ 2,4-Dinitrotoluene } & BTM & 1146 & 34614 & A & 1386 & 47 & & & & & & 1383 & 54 \\
\hline & & & & & 1384 & 46 & 4-Chlorotoluene & WWR & 1696 & 77277 & A & 1390 & 56 \\
\hline 2,4-Dinitrotoluene & WWR & 1091 & 34611 & A & 1394 & 58 & 4-Chlorotoluene & WWR & 1482 & 77277 & B & 1380 & 53 \\
\hline & & & & & 1385 & 55 & & & & & & 1392 & 57 \\
\hline & & & & & 1383 & 54 & 4-Nitrophenol & BTM & 1051 & 34649 & A & 1386 & 47 \\
\hline 2,4-DP & BTM & 403 & -- & A & 1305 & 45 & & & & & & 1384 & 46 \\
\hline & & & & & 80 & 45 & 4-Nitrophenol & WWR & 1063 & 34646 & $\mathbf{A}$ & 1385 & 55 \\
\hline 2,4-DP & DIS & 487 & 82356 & A & 1301 & 49 & & & & & & 1393 & 57 \\
\hline 2,4-DP & WWR & 402 & 82183 & $\mathbf{A}$ & 1474 & 59 & & & & & & 1383 & 54 \\
\hline & & & & & 79 & 49 & 9H-carbazole & BTM & 528 & & B & 2502 & 48 \\
\hline & & & & & 1304 & 49 & $9 \mathrm{H}$-fluorene & BTM & 5210 & --1 & B & 2502 & 48 \\
\hline 2,6-Dinitrotoluene & BTM & 114 & 34629 & $A$ & 1386 & 47 & 9H-fluorene, 1-methyl & BTM & 5273 & --1 & B & 2502 & 48 \\
\hline & & & & & 1384 & 46 & Acenaphthene & BTM & 1112 & 34208 & A & 1386 & 47 \\
\hline 2,6-Dinitrotoluene & WWR & 1092 & 34626 & A & 1394 & 58 & & & & & & 1384 & 46 \\
\hline & & & & & 1385 & 55 & Acenaphthene & BTM & 5211 & & B & 2502 & 48 \\
\hline & & & & & 1383 & 54 & Acenaphthene & WWR & 1066 & 34205 & $\mathbf{A}$ & 1394 & 58 \\
\hline 2-Chloroethylvinylether & WWR & 1017 & 34576 & $A$ & 1390 & 56 & & & & & & 1385 & 55 \\
\hline 2-Chloroethylvinylether & WWR & 1658 & 34576 & C & 1380 & 53 & & & & & & 1383 & 54 \\
\hline & & & & & 1392 & 57 & Acenaphthylene & BTM & 113 & 34203 & A & 1386 & 47 \\
\hline & & & & & 1401 & 59 & & & & & & 1384 & 46 \\
\hline 2-Chloronaphthalene & BTM & 1126 & 34584 & A & 1386 & 47 & Acenaphthylene & BTM & 5212 & & B & 2502 & 48 \\
\hline & & & & & 1384 & 46 & Acenaphthylene & WWR & 1067 & 34200 & A & 1394 & 58 \\
\hline 2-Chloronaphthalene & WWR & 1080 & 34581 & A & 1394 & 58 & & & & & & 1385 & 55 \\
\hline & & & & & 1385 & 55 & & & & & & 1383 & 54 \\
\hline & & & & & 1383 & 54 & Acifluorfen (Scepter) & WWR & 5410 & & A & 2050 & 61 \\
\hline 2-Chlorophenol & BTM & 1045 & 34589 & A & 1386 & 47 & & & 5610 & -. & A & 2051 & 61 \\
\hline 2-Chlorophenol & BTM & 1045 & 34590 & $\mathbf{A}$ & 1384 & 46 & Acrolein & WWR & 1650 & 34210 & A & 1390 & 56 \\
\hline 2-Chlorophenol & WWR & 1056 & 34586 & A & 1385 & 59 & & & & & & 1380 & 53 \\
\hline & & & & & 1393 & 57 & & & & & & 1392 & 57 \\
\hline & & & & & 1383 & 54 & & & & & & 1401 & 59 \\
\hline 2-Chlorotoluene & WWR & 1694 & 77275 & & 1390 & 56 & Acridine & BTM & 5276 & & B & 2502 & 48 \\
\hline 2-Chlorotoluene & WWR & 1481 & 77275 & & 1380 & 53 & Acrylonitrile & WWR & 1651 & 34215 & A & 1390 & 56 \\
\hline & & & & & 1392 & 57 & & & & & & 1380 & 53 \\
\hline 2-Methyl-4,6-dinitropheonol & BTM & 1048 & 34660 & A & 1386 & 47 & & & & & & 1392 & 57 \\
\hline & & & & & 1384 & 46 & & & & & & 1401 & 59 \\
\hline 2-Methyle-4,6-dinitropheonol & & 1060 & 34657 & $\mathbf{A}$ & 1385 & 46 & Alachlor & DIS & 1587 & 46342 & A & 1379 & 53 \\
\hline
\end{tabular}




\begin{tabular}{l} 
Organic parameters--Continued \\
\hline Alachlor \\
Alachlor \\
Aldicarb \\
Aldicarb \\
Aldicarb sulfone \\
Aldicarbsulfone \\
Aldicarb sulfoxide \\
Aldicarb solfoxide \\
Aldrin \\
Aldrin \\
Aldrin \\
Aldrin \\
Aldrin \\
Aldrin \\
Alpha BHC \\
Ametryn \\
Ametryn \\
Amine, $n$-Nitroso-Di-n-Propyl \\
Anthracene \\
Anthacene
\end{tabular}

Anthracene, 2-methyl

Anthraquinone

Aroclor 1016

Aroclor 1016

Aroclor 1016

Aroclor 1221

Aroclor 1221

Aroclor 1221

Aroclor 1232

Arocior 1232

Aroclor 1232

Aroclor 1242

Aroclor 1242

Aroclor 1242

Aroclor 1248

Aroclor 1248

Aroclor 1248

Aroclor 1254

Aroclor 1254

Aroclor 1254

Aroclor 1260

Aroclor 1260

Aroclor 1260

Atrazine

Atrazine

Atrazine

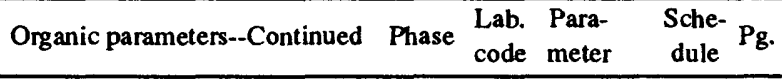

4203 39632 E 201060

Atrazine, desethyl-

WWR 400204040 D 200160 420204040 E 201060

Azinphos, methyl-

$\begin{array}{llllll}\text { WWR } & 1338 & 82619 & C & 1359 & 52\end{array}$

WWR $5411 \quad$ - A 205061 $\begin{array}{lllll}5611 & - \text { A } 205161\end{array}$

$\begin{array}{llllll}\text { WWR } & 1344 & 82587 & C & 1359 & 52\end{array}$

WWR $5413 \quad$ - A 205061 $\begin{array}{llll}5613 & - \text { A } & 2051 \quad 61\end{array}$

$\begin{array}{llllll}\text { WWR } & 1343 \quad 82586 & C & 1359 & 52\end{array}$

WWR $5412 \quad$-. A 205061

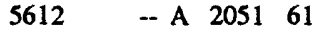

BTM 36139333 A $1335 \quad 45$ $1325 \quad 45$ $\begin{array}{lllll}5001 & \text { - } & \text { B } & 2501 & 47\end{array}$ DIS $\quad \begin{array}{llllll}463 & 39331 & \text { A } & 1331 & 51\end{array}$ 132150

$\begin{array}{lllll}\text { TIS } & 7030 & \text {-. A } & 2101 \quad 63\end{array}$ $\begin{array}{lllllll}\text { WWR } & 738 & 39330 & \text { B } & 1399 & 58\end{array}$ $1398 \quad 58$

$\begin{array}{llllll}\text { WWR } & 350 & 39330 & \mathrm{C} & 1474 & 59\end{array}$ 133452 132450

$\begin{array}{llllll}\text { WWR } & 1624 & 39330 & \mathrm{D} & 1608 & 59\end{array}$ $\begin{array}{llllll}\text { WWR } & 1619 & 39337 & \text { D } & 1608 & 59\end{array}$ $\begin{array}{llllll}\text { DIS } & 1588 & 38401 & \text { A } & 1379 & 53\end{array}$ $\begin{array}{lllllll}\text { WWR } & 848 & 82184 & \text { A } & 1389 & 56\end{array}$ $\begin{array}{llllll}\text { BTM } & 5245 & \text {-- B } & 2502 & 48\end{array}$ $\begin{array}{llllll}\text { BTM } 5244 & \text {-- B } 2502 \quad 48\end{array}$ BTM $1114 \quad 34223$ A $1386 \quad 47$ 138446 $\begin{array}{lllll}\text { BTM } & 5213 & \text { - B } & 2502 & 48\end{array}$ $\begin{array}{llllll}\text { WWR } & 1068 & 34220 & \text { A } & 1385 & 55\end{array}$ $1383 \quad 54$ 139458

$\begin{array}{llllll}\text { BTM } & 5279 & -- & \text { B } & 2502 & 48\end{array}$ $\begin{array}{lllll}\text { BTM } & 5283 & \text { - } & \text { B } 2502 & 48\end{array}$ $\begin{array}{lllllll}\text { DIS } & 787 & 34672 & \text { A } & 1361 & 52\end{array}$ $\begin{array}{lllllll}\text { WWR } & 809 & 34671 & \text { B } & 1364 & 52\end{array}$ $\begin{array}{lllllll}\text { WWR } & 1641 & 34671 & \mathrm{D} & 1608 & 59\end{array}$ $\begin{array}{lllllll}\text { DIS } & 783 & 34662 & \text { A } & 1361 & 52\end{array}$ $\begin{array}{lllllll}\text { WWR } & 810 & 39488 & \text { B } & 1364 & 52\end{array}$ $\begin{array}{lllllll}\text { WWR } & 1639 & 39488 & \mathrm{D} & 1608 & 59\end{array}$ $\begin{array}{lllllll}\text { DIS } & 779 & 34665 & \text { A } & 1361 & 52\end{array}$ $\begin{array}{llllll}\text { WWR } & 811 & 39492 & \text { B } & 1364 & 52\end{array}$ $\begin{array}{llllll}\text { WWR } & 1640 & 39492 & \mathrm{D} & 1608 & 59\end{array}$ $\begin{array}{llllll}\text { WWR } & 812 & 39496 & \text { B } & 1364 & 52\end{array}$ $\begin{array}{llllll}\text { WWR } & 1642 & 39496 & \text { D } & 1608 & 59\end{array}$ $\begin{array}{llllll}\text { DIS } & 775 & 34457 & \text { A } & 1361 & 52\end{array}$ $\begin{array}{lllllll}\text { DIS } & 771 & 39501 & \text { A } & 1361 \quad 52\end{array}$ $\begin{array}{llllll}\text { WWR } & 813 & 39500 & \text { B } & 1364 & 52\end{array}$ $\begin{array}{llllll}\text { WWR } & 1643 & 39500 & \mathrm{D} & 1608 & 59\end{array}$ $\begin{array}{llllll}\text { DIS } & 767 & 39505 \text { A } & 1361 & 52\end{array}$ $\begin{array}{llllll}\text { WWR } & 814 & 39504 & \text { B } & 1364 & 52\end{array}$ $\begin{array}{llllll}\text { WWR } & 1644 & 39504 & \mathrm{D} & 1608 & 59\end{array}$ $\begin{array}{lllllll}\text { DIS } & 763 & 39509 & \text { A } & 1361 & 52\end{array}$ $\begin{array}{llllll}\text { WWR } & 815 & 39508 & \text { B } & 1364 & 52\end{array}$ $\begin{array}{llllll}\text { WWR } & 1645 & 39508 & \mathrm{D} & 1608 & 59\end{array}$ DIS $\quad \begin{array}{llllll}1589 & 39632 & \text { A } & 1379 \quad 53\end{array}$ $\begin{array}{llllll}\text { WWR } & 717 & 39630 & \text { A } & 1389 & 56\end{array}$ WWR 400339632 D 200160
Azobenzene

Benfluralin

Bentazon

Benzene, 1-chloro-2-methylBenzene, 1-chloro-4-methylBenzene, 1-methyl-4-isopropylBenzene, 1,2-dichloro Benzene, 1,2-dichloroBenzene, 1,2,3-TrichloroBenzene, 1,2,4-dichloro Benzene, 1,2,4-TrichloroBenzene, 1,2,4-TrimethylBenzene, 1,3-dichloro Benzene, 1,3-trimethylBenzene, 1,3,5-trimethylBenzene, 1,4-dichloro Benzene, 1,4-dichloroBenzene Benzene

Benzene

Benzene, bromo-

Benzene, chloro-

Benzene, dimethyl- (Xylene)

Benzene, ethyl-

Benzene, isopropyl-

Benzene, methyl (Toluene)

Benzene, n-butyl-

Benzene, n-propyl

Benzene, nitro

Benzene, nitro, d5,-surrogate \%

Benzene, pentachloro-

Benzene, pentachloronitro-

Benzene, sec-butyl

Benzidine

Benzo[a]anthracene

Benzo[a]pyrene

Benzo[a]pyrene

Benzo[a]pyrene

Benzo[b]fluoranthene

Benzo[b]fluoranthene

Benzo[b]fluoranthene

Benzo[c]quinoline
WWR 400482686 D 200160 420482686 E 201060

$\begin{array}{lllll}\text { BTM } 5272 & - \text { - B } 250248\end{array}$

WWR 400582673 D 200160 420582673 E 201060

WWR 541438711 A 205061 $5614 \quad 38711$ A $2051 \quad 61$

WWR $5844 \quad 77275$ C $2090 \quad 62$ WWR $5858 \quad 77277$ C $2090 \quad 62$ $\begin{array}{llllll}\text { WWR } & 5848 & 7356 & \text { B } & 2090 & 62\end{array}$ $\begin{array}{llllll}\text { BTM } 5234 & \text {-- B } 250248\end{array}$ WWR $5852 \quad 34536$ D 209062 WWR $5857 \quad 77613$ C $2090 \quad 62$ $\begin{array}{llllll}\text { BTM } & 5201 & \text { - B } & 2502 \quad 48\end{array}$ WWR $5854 \quad 34551$ D $2090 \quad 62$ WWR $5846 \quad 77222$ C $2090 \quad 62$ $\begin{array}{lllll}\text { BTM } 5222 & \text {-- B } & 2502 & 48\end{array}$ $\begin{array}{llllll}\text { WWR } & 5849 & 34586 & C & 2090 & 62\end{array}$ WWR $5860 \quad 77226$ C $2090 \quad 62$ $\begin{array}{lllll}\text { BTM } 5233 & \text {-- B } 250248\end{array}$ $\begin{array}{llllll}\text { WWR } & 5850 & 34571 & \text { D } & 2090 & 62\end{array}$ $\begin{array}{lllllll}W^{\prime \prime} & 1011 & 34030 & \text { A } & 1390 & 56\end{array}$ $\begin{array}{lllllll}\text { WWR } & 1287 & 34030 & \text { B } & 1307 \quad 49\end{array}$ $1378 \quad 54$ $1380 \quad 53$ $1387 \quad 55$ 139257

$\begin{array}{llllll}\text { WWR } & 5819 & 34030 & C & 2090 \quad 62\end{array}$ WWR $5841 \quad 81555$ C 209062 WWR $5833 \quad 34301$ C $2090 \quad 62$ $\begin{array}{lllllll}\text { WWR } & 5836 & 81551 & C & 2090 & 62\end{array}$ $\begin{array}{llllll}\text { WWR } & 5835 & 34371 & C & 2090 & 62\end{array}$ $\begin{array}{llllll}\text { WWR } & 5839 & 7223 & C & 2090 & 62\end{array}$ WWR $5826 \quad 34010$ C $2090 \quad 62$ WWR $5851 \quad 77342$ C $2090 \quad 62$ WWR $5843 \quad 77224$ C 209062 $\begin{array}{llllll}\text { BTM } & 5247 & \text { - } & \text { B } & 2502 & 48\end{array}$ $\begin{array}{llllll}\text { BTM } & 5287 & \text {-- B } & 2502 & 48\end{array}$ $\begin{array}{llllll}\text { BTM } 5228 & \text { - } & \text { B } & 2502 & 48\end{array}$ $\begin{array}{lllll}\text { BTM } 5226 & \text { - } & \text { B } 2502 & 48\end{array}$ WWR $5847 \quad 77350$ C $2090 \quad 62$ 584577353 C 209062 WWR 106939120 A $1385 \quad 55$ 138354

$\begin{array}{llllll}\text { BTM } & 5217 & \text { - } & \text { B } & 2502 & 48\end{array}$ $\begin{array}{llllll}\text { BTM } & 5221 & \text { - } & \text { B } & 2502 & 48\end{array}$ $\begin{array}{llllll}\text { BTM } & 1119 & 34250 & \text { A } & 1386 & 47\end{array}$ $1384 \quad 46$ 138555 $1383 \quad 54$

$\begin{array}{llllll}\text { BTM } & 5218 & \text { - } & \text { B } & 2502 & 48\end{array}$ BTM $1117 \quad 34233$ A $1386 \quad 47$ $1384 \quad 46$ $\begin{array}{lllll}\text { WWR } & 1071 \quad 34230 \text { A } 1385 \quad 55\end{array}$ $1383 \quad 54$ 139458 $\begin{array}{llllll}\text { BTM } & 5280 & \text {-- B } & 2502 & 48\end{array}$ $\begin{array}{llllll}\text { WWR } & 1073 \quad 34247 & \text { A } & 1394 \quad 58\end{array}$ 


\begin{tabular}{|c|c|c|c|c|c|c|c|c|c|c|c|c|}
\hline Organic parameters--Continued & Phase & $\begin{array}{l}\text { Lab. } \\
\text { code }\end{array}$ & $\begin{array}{l}\text { Para- } \\
\text { meter }\end{array}$ & $\begin{array}{l}\text { Sche- } \\
\text { dule }\end{array}$ & Pg. & Organic parameters--Continued & Phase & $\begin{array}{l}\text { Lab. } \\
\text { code }\end{array}$ & $\begin{array}{l}\text { Para- } \\
\text { meter }\end{array}$ & & $\begin{array}{l}\text { Sche- } \\
\text { dule }\end{array}$ & Pg. \\
\hline Benzolg,h,1]perylene- & BTIM & 5219 & $\because \mathrm{B}$ & 2502 & 48 & Butyl benzyl phthalate & BTM & 1121 & 34295 & A & 1386 & $\overline{4 T}$ \\
\hline Benzo[k]fluoranthene & BTM & 5220 & $--\mathrm{B}$ & 2502 & 48 & & & & & & 1384 & 46 \\
\hline \multirow[t]{2}{*}{ Benzo[k]fluoranthene } & BTM & 1118 & 34245 A & 1386 & 47 & Butyl benzyl phthalate & WWR & 1075 & 34292 & $\mathbf{A}$ & 1394 & 58 \\
\hline & & & & 1384 & 46 & & & & & & 1385 & 55 \\
\hline \multirow[t]{3}{*}{ Benzo[k]fluoranthene } & WWR & 1072 & 34242 A & 1394 & 58 & & & & & & 1383 & 54 \\
\hline & & & & 1385 & 55 & Butylate & WWR & 1470 & 30236 & $A$ & 1389 & 56 \\
\hline & & & & 1383 & 54 & & & 4006 & 04028 & D & 2001 & 60 \\
\hline \multirow[t]{2}{*}{ Benzo[a]anthmcene } & BTM & 1116 & 34529 A & 1386 & 47 & & & 4206 & 04028 & $\mathbf{E}$ & 2010 & 60 \\
\hline & & & & 1384 & 46 & Carbaryl (Sevin) & WWR & 636 & 39750 & $\mathrm{~A}$ & 1359 & 52 \\
\hline \multirow[t]{3}{*}{ Benzo[a]anthracene } & WWR & 1070 & $34526 \mathrm{~A}$ & 1385 & 55 & & & 4007 & 82680 & $D$ & 2001 & 60 \\
\hline & & & & 1383 & 54 & & & 4207 & 82680 & $\mathbf{E}$ & 2010 & 60 \\
\hline & & & & 1394 & 58 & Carbaryl (Sevin) & WWR & 5417 & & A & 2050 & 61 \\
\hline \multirow[t]{2}{*}{ Benzo[g,h,i]perylene } & BTM & 1120 & 34524 A & 1386 & 47 & & & 5617 & & $\mathbf{A}$ & 2051 & 61 \\
\hline & & & & 1384 & 46 & Carbofuran & WWR & 1337 & 82615 & $\mathrm{C}$ & 1359 & 52 \\
\hline \multirow{3}{*}{ Benzo[g,h,i]perylene } & WWR & 1074 & $34521 \mathrm{~A}$ & 1385 & 55 & & & 4008 & 82674 & D & 2001 & 60 \\
\hline & & & & 1383 & 54 & & & 4208 & 82674 & E & 2010 & 60 \\
\hline & & & & 1394 & 58 & Carbofuran & WWR & 5418 & & $A$ & 2050 & 61 \\
\hline Beta BHC & WWR & 1620 & $39338 \mathrm{D}$ & 1608 & 59 & & & 5618 & & $A$ & 2051 & 61 \\
\hline Biphenyl,, 2-chloro & BTM & 5206 & $--\mathrm{B}$ & 2502 & 48 & Carbofuran, 3-hydroxy- & WWR & 5449 & & A & 2050 & 61 \\
\hline Biphenyl, 2-fluoro-surrogate \% & BTM & 5288 & $--\mathrm{B}$ & 2502 & 48 & & & 5649 & -- & $A$ & 2051 & 61 \\
\hline Biphenyl,3,5,-dichloro & BTM & 5034 & $--\mathbf{B}$ & 2501 & 47 & Carbon, inorganic & BTM & 503 & 686 & $\mathrm{C}$ & - & 43 \\
\hline \multirow[t]{2}{*}{ Bis (2-chloroethoxy) } & BTM & 1122 & $34281 \mathrm{~A}$ & 1386 & 47 & Carbon, inorganic & DIS & 306 & 691 & $A$ & - & 43 \\
\hline & & & & 1384 & 46 & Carbon, inorganic & Total & 19 & 685 & A & - & 43 \\
\hline \multirow[t]{3}{*}{ Bis (2-chloroethoxy)methane } & WWR & 1076 & 34278 A & 1385 & 55 & Carbon, organic & DIS & 113 & 681 & $\mathbf{A}$ & -- & 43 \\
\hline & & & & 1383 & 54 & & & & & & 2085 & 62 \\
\hline & & & & 1394 & 58 & & & & & & 2075 & 62 \\
\hline \multirow[t]{2}{*}{ Bis (2-chloroethyl) ether } & BTM & 1123 & 34276 A & 1386 & 47 & Carbon, organic & SUS & 305 & 689 & $A$ & - & 43 \\
\hline & & & & 1384 & 46 & & & & & & 2075 & 62 \\
\hline \multirow[t]{3}{*}{ Bis (2-chloroethyl) ether } & WWR & 1077 & 34273 A & 1385 & 55 & Carbon, organic & Total & 114 & 680 & $A$ & -- & 43 \\
\hline & & & & 1383 & 54 & Carbon, total & BTM & 133 & 693 & $\mathbf{A}$ & -- & 43 \\
\hline & & & & 1394 & 58 & Carbontetrachloride & WWR & 1013 & 32102 & $\mathbf{A}$ & 1390 & 56 \\
\hline \multirow[t]{2}{*}{ Bis (2-chloroisopropyl) ether } & BTM & 1124 & 34286 A & 1386 & 47 & Carbontetrachloride & WWR & 1289 & 32102 & B & 1307 & 49 \\
\hline & & & & 1384 & 46 & & & & & & 1380 & 58 \\
\hline \multirow[t]{3}{*}{ Bis (2-chlomisopropyl) ether } & WWR & 1078 & $34283 \mathrm{~A}$ & 1385 & 55 & & & & & & 1392 & 57 \\
\hline & & & & 1383 & 54 & Carboxin & WWR & 1464 & 30245 & $\mathbf{A}$ & 1389 & 56 \\
\hline & & & & 1394 & 58 & Chloramben (Amiben) & WWR & 5419 & -- & $\mathbf{A}$ & 2050 & 61 \\
\hline Bis (2-ethylhexyl) phthalate & BTM & 1149 & $39102 \mathrm{~A}$ & 1386 & 47 & & & 5619 & -- & $\mathbf{A}$ & 2051 & 61 \\
\hline & & & & 1384 & 46 & Chlordane & BTM & 362 & 39351 & $\mathbf{A}$ & 1335 & 45 \\
\hline Bis (2-ethylhexyl) phthalate & WWR & 1094 & $39100 \mathrm{~A}$ & 1385 & 55 & & & & & & 1325 & 45 \\
\hline & & & & 1383 & 54 & Chlordane & DIS & 464 & 39352 & $\mathbf{A}$ & 1331 & 51 \\
\hline & & & & 1394 & 58 & & & & & & 1321 & 50 \\
\hline Bromacil & WWR & 1463 & 30234 A & 1389 & 56 & Chlordane & WWR & 351 & 39350 & B & 1474 & 59 \\
\hline Bromacil & WWR & 5415 & 04029 A & 2050 & 61 & & & & & & 1399 & 58 \\
\hline & & 5615 & $04029 \mathrm{~A}$ & 2051 & 61 & & & & & & 1334 & 52 \\
\hline Bromobenzene & WWR & 1698 & $81555 \mathrm{~A}$ & 1380 & 53 & & & & & & 1398 & 58 \\
\hline & & & & 1392 & 57 & & & & & & 1324 & 51 \\
\hline Bromobenzene & WWR & 1485 & 81555 B & 1390 & 56 & Chlordane, cis & BTM & 5002 & - & B & 2501 & 47 \\
\hline Bromochloromethane & WWR & 1654 & 77297 A & 1380 & 53 & Clhordane-cis & TIS & 7001 & -- & $\mathbf{A}$ & 2101 & 63 \\
\hline & & & & 1392 & 57 & Chlordane, cis & WWR & 1628 & 39065 & D & 1608 & 59 \\
\hline Bromochloromethane & WWR & 1655 & 77297 B & 1390 & 56 & Chlordane, trans & BTM & 5003 & -- & B & 2501 & 47 \\
\hline Bromoform & WWR & 1012 & $32104 \mathrm{~A}$ & 1390 & 56 & Chlordane-trans & TIS & 7002 & - & $\mathbf{A}$ & 2101 & 63 \\
\hline Bromoform & WWR & 1288 & $32104 \mathrm{~B}$ & 1307 & 49 & Chlordane, trans & WWR & 1626 & 39062 & $\mathrm{D}$ & 1608 & 59 \\
\hline & & & & 1380 & 53 & Chlorobenzene & WWR & 1014 & 34301 & A & 1390 & 56 \\
\hline & & & & 1387 & 55 & Chlorobenzene & WWR & 1290 & 34301 & B & 1307 & 49 \\
\hline & & & & 1392 & 57 & & & & & & 1380 & 53 \\
\hline Bromomethane & WWR & 1028 & 34413 A & 1390 & 56 & & & & & & 1392 & 57 \\
\hline Bromomethane & WWR & 1304 & $34413 \mathrm{~B}$ & 1380 & 53 & Chloroethane & WWR & 1016 & 34311 & $\mathbf{A}$ & 1390 & 56 \\
\hline & & & & 1392 & 57 & Chloroethane & WWR & 1292 & 34311 & B & 1380 & 53 \\
\hline Bromozynil & WWR & 5416 & $-A$ & 2050 & 61 & & & & & & 1392 & 57 \\
\hline & & 5616 & $-A$ & 2051 & 61 & Chloroform & WWR & 1018 & 32106 & $\mathbf{A}$ & 1390 & 56 \\
\hline Butachlor & WWR & 1468 & $30235 \mathrm{~A}$ & 1389 & 56 & Chloroform & WWR & 1294 & 32106 & $\mathrm{~B}$ & 1307 & 49 \\
\hline
\end{tabular}




\begin{tabular}{|c|c|c|c|c|c|c|c|c|c|c|c|c|}
\hline Organic parameters--Continued & Phase & $\begin{array}{l}\text { Lab. } \\
\text { code }\end{array}$ & $\begin{array}{l}\text { Para- } \\
\text { meter }\end{array}$ & & $\begin{array}{l}\text { Sche- } \\
\text { dule }\end{array}$ & Pg. & Organic parameters--Continued & Phase & $\begin{array}{l}\text { Lab. } \\
\text { code }\end{array}$ & $\begin{array}{l}\text { Para- } \\
\text { meter }\end{array}$ & $\begin{array}{l}\text { Sche- } \\
\text { dule }\end{array}$ & Pg. \\
\hline & & & & & 1380 & $\overline{53}$ & DDE,p,p'- & $\overline{\text { BTM }}$ & 5णा & $\ldots$ & 250 & $\overline{4 T}$ \\
\hline & & & & & 1387 & 55 & DDE,p,p'- & WWR & 4012 & 34653 I & 2001 & 60 \\
\hline & & & & & 1392 & 57 & & & 4212 & 34653 & 2010 & 60 \\
\hline Chloromethane & WWR & 1281 & 34418 & $\mathbf{A}$ & 1390 & 56 & DDT & BTM & 365 & $39373 A$ & 1325 & 45 \\
\hline \multirow[t]{2}{*}{ Chloromethane } & WWR & 1318 & 34418 & B & 1380 & 53 & & & & & 1335 & 45 \\
\hline & & & & & 1392 & 57 & DDT & DIS & 467 & $39371 A$ & 1331 & 51 \\
\hline Chloroneb & BTM & 5054 & -- & B & 2501 & 47 & & & & & 1321 & 50 \\
\hline \multirow[t]{6}{*}{ Chloropyrifos } & WWR & 753 & 38932 & $\mathbf{A}$ & 1474 & 59 & DDT & WWR & 741 & $39370 \mathrm{E}$ & 31399 & 58 \\
\hline & & & & & 1334 & 52 & & & & & 1398 & 58 \\
\hline & & & & & 1319 & 50 & DDT & WWR & 354 & 39370 & 1474 & 59 \\
\hline & & & & & 1386 & 58 & & & & & 1334 & 52 \\
\hline & & 4009 & 38933 & D & 2001 & 60 & & & & & 1324 & 51 \\
\hline & & 4209 & 38933 & $\mathbf{E}$ & 2010 & 60 & DDT,o,p' & BTM & 5012 & $--\mathrm{E}$ & 2501 & 47 \\
\hline \multirow[t]{2}{*}{ Chlorothalonil } & WWR & 5421 & -. & $\mathbf{A}$ & 2050 & 61 & DDT,o,p' & TIS & 7004 & $-A$ & 2101 & 63 \\
\hline & & 5621 & -- & $\mathbf{A}$ & 2051 & 61 & DDT,p,p' & BTM & 5013 & -- & A 2501 & 47 \\
\hline Chrysene & BTM & 5225 & -- & B & 2502 & 48 & DDT,p,p' & TIS & 7005 & $\cdots$ & A 2101 & 63 \\
\hline Chrysene & BTM & 1128 & 34323 & $\mathbf{A}$ & 1384 & 46 & DNOC & WWR & 5402 & -- & 2050 & 61 \\
\hline \multirow[t]{3}{*}{ Chrysene } & WWR & 1082 & 34320 & $\mathbf{A}$ & 1394 & 58 & & & 5602 & - & 2051 & 61 \\
\hline & & & & & 1385 & 55 & Dacthal & TIS & 7003 & - & 2101 & 63 \\
\hline & & & & & 1383 & 54 & Dacthal, mono-acid- & WWR & 5447 & $\ldots$ & 2050 & 61 \\
\hline \multirow[t]{3}{*}{ Cis-1,2-dichloroethene } & WWR & 1656 & 77093 & A & 1307 & 49 & & & 5647 & $\ldots$ & 2051 & 61 \\
\hline & & & & & 1380 & 53 & De-ethylatrazine & DIS & 1519 & 04040 & 1379 & 53 \\
\hline & & & & & 1392 & 57 & De-ethylatrazine & WWR & 1612 & 75981 & A 1389 & 56 \\
\hline Cis-1,2-dichloroethene & WWR & 1657 & 77093 & B & 1390 & 56 & De-isopropylatrazine & DIS & 1592 & 04038 & A 1379 & 53 \\
\hline Cis-1,3-\&chloropropene & WWR & 1323 & 34704 & A & 1390 & 56 & De-isopropylatrazine & WWR & 1613 & 75980 & 1389 & 56 \\
\hline Cis-1,3-dichloropropene & WWR & 1326 & 34704 & B & 1380 & 53 & DEF & WWR & 802 & 39040 & 1474 & 59 \\
\hline Cis-1,3-dichloropropene & WWR & 1326 & 34704 & B & 1392 & 57 & & & & & 1334 & 52 \\
\hline \multirow[t]{2}{*}{ Clopyralid } & WWR & 5423 & -- & $\mathbf{A}$ & 2050 & 61 & & & & & 1319 & 50 \\
\hline & & 5623 & -- & A & 2051 & 61 & Delta-BHC & WWR & 1622 & 34259 I & 1608 & 59 \\
\hline Cyanazine & DIS & 1590 & 04041 & $\mathbf{A}$ & 1379 & 53 & Di-n-butyl phthalate & BTM & 1130 & 39112 & 1386 & 47 \\
\hline \multirow[t]{3}{*}{ Cyanazine } & WWR & 846 & 81757 & $\mathbf{A}$ & 1389 & 56 & & & & & 1384 & 46 \\
\hline & & 4010 & 04041 & D & 2001 & 60 & Di-n-butyl phthalate & WWR & 1084 & 39110 & 1385 & 55 \\
\hline & & 4210 & 04041 & $\mathrm{E}$ & 2010 & 60 & & & & & 1383 & 54 \\
\hline Cycloate & WWR & 1469 & 30254 & A & 1389 & 56 & & & & & 1394 & 58 \\
\hline DCPA (Dacthal) & BTM & 5036 & -. & B & 2501 & 47 & Di-n-octyl phthalate & BTM & 1149 & 34599 & 1386 & 47 \\
\hline \multirow[t]{2}{*}{ DCPA (Dacthal) } & WWR & 4011 & 82682 & D & 2001 & 60 & & & & & 1384 & 46 \\
\hline & & 4211 & 82682 & $\mathbf{E}$ & 2010 & 60 & Di-n-octyl phthalate & WWR & 1093 & 34596 & 1385 & 55 \\
\hline \multirow[t]{2}{*}{ DDD } & BTM & 363 & 39363 & $\mathbf{A}$ & 1325 & 45 & & & & & 1383 & 54 \\
\hline & & & & & 1335 & 45 & & & & & 1394 & 58 \\
\hline \multirow[t]{2}{*}{ DDD } & DIS & 465 & 39361 & $\mathbf{A}$ & 1331 & 51 & Di-Syston (disulfoton) & WWR & 592 & 39011 & 1319 & 50 \\
\hline & & & & & 1321 & 50 & & & & & 1334 & 52 \\
\hline \multirow[t]{2}{*}{ DDD } & WWR & 739 & 39361 & B & 1398 & 58 & & & & & 1474 & 59 \\
\hline & & & & & 1399 & 58 & Diazinon & BTM & 385 & 39571 & 1335 & 45 \\
\hline \multirow[t]{3}{*}{ DDD } & WWR & 352 & 39360 & $\mathbf{C}$ & 1474 & 59 & & & & & 1320 & 45 \\
\hline & & & & & 1334 & 52 & Diazinon & DIS & 423 & 39572 & 1331 & 51 \\
\hline & & & & & 1324 & 51 & & & & & 1321 & 50 \\
\hline DDD,o,p'- & BTM & 5008 & -- & B & 2501 & 47 & Diazinon & SUS & 417 & 39573 & A 1317 & 50 \\
\hline DDD,o,p' & TIS & 7007 & -- & $\mathbf{A}$ & 2101 & 63 & Diazinon & WWR & 378 & 39570 & B 1474 & 59 \\
\hline DDD,p,p'- & BTM & 5009 & -- & A & 2501 & 47 & & & & & 1399 & 58 \\
\hline DDD,p,p' & TIS & 7006 & - & A & 2101 & 63 & & & & & 1334 & 52 \\
\hline DDE & BTM & 364 & 39368 & $\mathbf{A}$ & 1325 & 45 & Diazinon & WWR & 378 & 39570 & B 1319 & 50 \\
\hline & & & & & 1335 & 45 & Diazinon & WWR & 4013 & 39572 & 2001 & 60 \\
\hline DDE & DIS & 466 & 39366 & A & 1331 & 51 & & & 4213 & 39572 & E 2010 & 60 \\
\hline & & & & & 1321 & 50 & Diazinon, d10-surrogate\% & WWR & 4014 & 910631 & 2001 & 60 \\
\hline DDE & WWR & 740 & 39365 & B & 1399 & 58 & & & 4214 & 91063 & E 2010 & 60 \\
\hline & & & & & 1398 & 58 & Dibenzo $[a, h]$ anthracene & BTM & 5232 & --1 & B 2502 & 48 \\
\hline DDE & WWR & 353 & 39365 & $\mathrm{C}$ & 1474 & 59 & Dibenzothiophene & BTM & 5275 & -1 & B 2502 & 48 \\
\hline & & & & & 1334 & 52 & Dibromochloromethane & WWR & 1015 & 32105 & A 1390 & 56 \\
\hline & & & & & 1324 & 51 & Dibromochloromethane & WWR & 1291 & 32105 & B 1307 & 49 \\
\hline DDE,o,p'- & BTM & 5010 & -- & B & 2501 & 47 & & & & & 1380 & 53 \\
\hline DDE,o,p' & TIS & 7008 & -- & A & 2101 & 63 & & & & & 1387 & 55 \\
\hline
\end{tabular}




\begin{tabular}{|c|c|c|c|c|c|c|c|c|c|c|c|c|c|}
\hline Organic parameters--Continued & Phase & $\begin{array}{l}\text { Lab. } \\
\text { code }\end{array}$ & $\begin{array}{l}\text { Para- } \\
\text { meter }\end{array}$ & & $\begin{array}{l}\text { Sche- } \\
\text { dule }\end{array}$ & Pg. & Organic parameters--Continued & Phase & $\begin{array}{l}\text { Lab. } \\
\text { code }\end{array}$ & $\begin{array}{l}\text { Para- } \\
\text { meter }\end{array}$ & & $\begin{array}{l}\text { Sche- } \\
\text { dule }\end{array}$ & Pg. \\
\hline & & & & & 1392 & $\overline{5 T}$ & $\overline{\text { Endosulfan }}$ & WWR & $73 T$ & 39388 & & T399 & $\overrightarrow{38}$ \\
\hline Dibromomethane & WWR & 1690 & 30217 & & 1390 & 56 & & & & & & 1398 & 58 \\
\hline \multirow[t]{2}{*}{ Dibromomethane } & WWR & 1009 & 30217 & B & 1380 & 53 & Endosulfan 1 & WWR & 349 & 39388 & C & 1474 & 59 \\
\hline & & & & & 1392 & 57 & & & & & & 1334 & 52 \\
\hline Dicamba & BTM & 751 & 38931 & $\mathbf{A}$ & 80 & 45 & & & & & & 1324 & 51 \\
\hline Dicamba & WWR & 749 & 82052 & $\mathbf{A}$ & 79 & 49 & Endosulfan (I) & BTM & 5015 & -- & B & 2501 & 47 \\
\hline \multirow[t]{2}{*}{ Dicamba } & WWR & 5426 & 38442 & $\mathbf{A}$ & 2050 & 61 & Endosulfan alpha (I) & WWR & 1627 & 34361 & D & 1608 & 59 \\
\hline & & 5626 & 38442 & $\mathbf{A}$ & 2051 & 61 & Endrin & BTM & 5018 & & B & 2501 & 47 \\
\hline \multirow[t]{2}{*}{ Dichlobenil } & WWR & 5404 & - & A & 2050 & 61 & Endrin & BTM & 367 & 39393 & A & 1335 & 45 \\
\hline & & 5604 & -- & $\mathbf{A}$ & 2051 & 61 & & & & & & 1325 & 45 \\
\hline Dichlorobromomethane & WWR & 1019 & 32101 & $\mathbf{A}$ & 1390 & 56 & Endrin & DIS & 469 & 39391 & A & 1331 & 51 \\
\hline \multirow[t]{3}{*}{ Dichlorobromomethane } & WWR & 1295 & 32101 & B & 1307 & 49 & & & & & & 1321 & 50 \\
\hline & & & & & 1387 & 55 & Endrin & TIS & 7011 & & A & 2101 & 63 \\
\hline & & & & & 1392 & 57 & Endrin & WWR & 743 & 39390 & B & 1399 & 58 \\
\hline Dichlorodifluoromethane & WWR & 1020 & 34668 & $\mathbf{A}$ & 1390 & 56 & & & & & & 1398 & 58 \\
\hline \multirow[t]{3}{*}{ Dichlorodifluoromethane } & WWR & 1296 & 34668 & B & 1307 & 49 & Endrin & WWR & 356 & 29290 & C & 1474 & 59 \\
\hline & & & & & 1380 & 53 & & & & & & 1334 & 52 \\
\hline & & & & & 1392 & 57 & & & & & & 1324 & 51 \\
\hline \multirow[t]{2}{*}{ Dichlorprop (2,4-DP) } & WWR & 5401 & & $\mathbf{A}$ & 2050 & 61 & Enthion & BTM & 386 & 39399 & A & 1335 & 45 \\
\hline & & 5601 & -- & $\mathbf{A}$ & 2051 & 61 & & & & & & 1320 & 45 \\
\hline \multirow[t]{2}{*}{ Dieldrin } & BTM & 366 & 39383 & A & 1335 & 45 & Enthion & DIS & 424 & 82346 & A & 1331 & 49 \\
\hline & & & & & 1325 & 45 & & & & & & 1316 & 50 \\
\hline Dieldrin & BTM & 5014 & -- & B & 2501 & 47 & Enthion & SUS & 418 & 82347 & A & 1317 & 50 \\
\hline \multirow[t]{2}{*}{ Dieldrin } & DIS & 468 & 39381 & $\mathbf{A}$ & 1331 & 51 & Enthion & WWR & 379 & 39398 & B & 1474 & 59 \\
\hline & & & & & 1321 & 50 & & & & & & 1399 & 58 \\
\hline Dieldrin & TIS & 7010 & & $\mathbf{A}$ & 2101 & 63 & & & & & & 1334 & 52 \\
\hline \multirow[t]{2}{*}{ Dieldrin } & WWR & 742 & 39380 & B & 1399 & 58 & & & & & & 1319 & 50 \\
\hline & & & & & 1398 & 58 & Ethalfluralin & WWR & 4020 & 82663 & $\mathrm{D}$ & 2001 & 60 \\
\hline \multirow[t]{3}{*}{ Dieldrin } & WWR & 355 & 39380 & $\mathbf{C}$ & 1474 & 59 & & & 4220 & 82663 & $\mathrm{E}$ & 2010 & 60 \\
\hline & & & & $\mathbf{C}$ & 1334 & 52 & Ethane, 1,1-dichloro- & WWR & 5811 & 34496 & C & 2090 & 62 \\
\hline & & & & C & 1324 & 51 & Ethane, 1,1,1-trichloro- & WWR & 5816 & 34506 & C & 2090 & 62 \\
\hline Dieldrin & WWR & 1629 & 39380 & $\mathrm{D}$ & 1608 & 59 & Ethane, 1,1,1,2-tetrachloro- & WWR & 5834 & 77562 & C & 2090 & 62 \\
\hline \multirow[t]{2}{*}{ Dieldrin } & WWR & 4015 & 39381 & $\mathrm{D}$ & 2001 & 60 & Ethane, 1,1,2-trichloro- & WWR & 5828 & 34511 & C & 2090 & 62 \\
\hline & & 4215 & 39381 & $\mathrm{D}$ & 2010 & 60 & Ethane, 1,1,2,2-tetrachloro- & WWR & 5840 & 34516 & C & 2090 & 62 \\
\hline Diethylphthalate & BTM & 1144 & 34339 & A & 1386 & 47 & Ethane, 1,2-dibromo-(EDB) & WWR & 5832 & 77651 & E & 2090 & 62 \\
\hline & & & & & 1384 & 46 & Ethane, 1,2-dichloro & WWR & 5820 & 32103 & C & 2090 & 62 \\
\hline Diethylphthalate & WWR & 1089 & 34336 & A & 1394 & 58 & Ethane, chloro & WWR & 5805 & 34311 & C & 2090 & 62 \\
\hline Diethylphthalate & WWR & 1089 & 34336 & A & 1385 & 55 & Ethane, hexachloro- & BTM & 5231 & -- & B & 2502 & 48 \\
\hline & & & & & 1383 & 54 & Ethane, trichlorotrifluoro- & WWR & 5859 & 77652 & C & 2090 & 62 \\
\hline Diethylaniline & WWR & 4016 & 82660 & D & 2001 & 60 & Ether, 4-bromophenylphenyl- & BTM & 5208 & -- & B & 2502 & 48 \\
\hline & & 4216 & 82660 & $\mathrm{E}$ & 2010 & 60 & Ether, 4-chlorophenylphenyl- & BTM & 5209 & -- & B & 2502 & 48 \\
\hline Dimethoate & WWR & 4017 & 82662 & $\mathrm{D}$ & 2001 & 60 & Ether, bis (2-chloroethyl)- & BTM & 5215 & -. & B & 2502 & 48 \\
\hline & & 4217 & 82662 & $\mathrm{E}$ & 2010 & 60 & Ether, bis (2-chloroisopropyl)- & BTM & 5216 & -- & B & 2502 & 48 \\
\hline Dimethyl phthalate & BTM & 1145 & 34344 & $\mathbf{A}$ & 1386 & 47 & Ether, Methyl tert-butyl (MTBE) & WWR & 5810 & 78032 & C & 2090 & 62 \\
\hline & & & & & 1384 & 46 & Ethoprop & WWR & 4021 & 82672 & D & 2001 & 60 \\
\hline Dimethyl phthalate & WWR & 1090 & 34341 & A & 1394 & 58 & & & 4221 & 82672 & $\mathrm{E}$ & 2010 & 60 \\
\hline & & & & & 1385 & 55 & Ethylene, 1,1-dichloro & WWR & 5807 & 34501 & C & 2090 & 62 \\
\hline & & & & & 1383 & 54 & Ethylene, chloro-(vinyl) & WWR & 5803 & 39175 & C & 2090 & 62 \\
\hline Dinoseb (DNBP) & WWR & 5400 & & $A$ & 2050 & 61 & & & & & & & \\
\hline & & 5600 & & $\mathbf{A}$ & 2051 & 61 & Ethylbenzene & WWR & 1027 & 34371 & A & 1390 & 56 \\
\hline Diphenamide & WWR & 1476 & 30255 & $A$ & 1389 & 56 & Ethylbenzene & WWR & 1303 & 34371 & B & 1307 & 49 \\
\hline Disulfoton & WWR & 4018 & 82677 & $\mathrm{D}$ & 2001 & 60 & & & & & & 1378 & 49 \\
\hline & & 4218 & 82677 & $E$ & 2010 & 60 & & & & & & 1380 & 53 \\
\hline Diuron & WWR & 5427 & -- & $A$ & 2050 & 61 & & & & & & 1387 & 55 \\
\hline & & 5627 & -- & $A$ & 2051 & 61 & & & & & & 1392 & 57 \\
\hline EPTC (Eptam) & WWR & 4019 & 82668 & D & 2001 & 60 & Ethylene, cis-1,2-dichloro & WWR & 5814 & 77093 & C & 2090 & 62 \\
\hline & & 4219 & 82668 & $\mathrm{E}$ & 2010 & 60 & Ethylene, tetrachloro & WWR & 5829 & 34475 & $\mathrm{C}$ & 2090 & 62 \\
\hline Endosulfan & BTM & 346 & 39389 & $A$ & 1335 & 45 & Ethylene, trans-1,2-dichloro & WWR & 5809 & 34546 & C & 2090 & 62 \\
\hline & & & & & 1325 & 45 & Ethylene, trichloro & WWR & 5821 & 39180 & C & 2090 & 62 \\
\hline Endosulfan & DIS & 345 & 82354 & A & 1331 & 51 & Esfenvalerate (Asana XL) & WWR & 5429 & & A & 2050 & 61 \\
\hline & & & & & 1321 & 50 & & & 5629 & -- & A & 2051 & 61 \\
\hline
\end{tabular}




\begin{tabular}{|c|c|c|c|c|c|c|c|c|c|c|c|c|}
\hline Organic parameters--Continued & Phase & $\begin{array}{l}\text { Lab. } \\
\text { code }\end{array}$ & $\begin{array}{l}\text { Para- } \\
\text { meter }\end{array}$ & $\begin{array}{l}\text { Sche- } \\
\text { dule }\end{array}$ & Pg. & Organic parameters--Continued & Phase & $\begin{array}{l}\text { Lab. } \\
\text { code }\end{array}$ & $\begin{array}{l}\text { Para- } \\
\text { meter }\end{array}$ & & $\begin{array}{l}\text { Sche- } \\
\text { dule }\end{array}$ & Pg. \\
\hline \multirow[t]{2}{*}{ Fenuron } & WWR & 5406 & $--A$ & 2050 & 61 & & & & & & 1394 & $\overline{46}$ \\
\hline & & 5606 & $--\mathbf{A}$ & 2051 & 61 & Hexachlorobenzene & BTM & 5006 & -- & B & 2501 & 47 \\
\hline \multirow[t]{2}{*}{ Flurmeturon } & WWR & 5430 & $38811 \mathrm{~A}$ & 2050 & 61 & Hexachlorobenzene & TIS & 7014 & -. & $\mathbf{A}$ & 2101 & 63 \\
\hline & & 5630 & $38811 \mathrm{~A}$ & 2051 & 61 & Hexachlorobenzene & WWR & 1097 & 39700 & $\mathbf{A}$ & 1385 & 55 \\
\hline Fluorenthene & BTM & 5240 & - B & 2502 & 48 & & & & & & 1383 & 54 \\
\hline \multirow{2}{*}{ Fluoranthene } & BTM & 1151 & 34379 A & 1386 & 47 & & & & & & 1394 & 58 \\
\hline & & & & 1384 & 46 & Hexachlorobutadiene & BTM & 1153 & 39705 & A & 1386 & 47 \\
\hline \multirow[t]{3}{*}{ Fluoranthene } & WWR & 1096 & 34376 A & 1394 & 58 & & & & & & 1384 & 46 \\
\hline & & & & 1385 & 55 & Hexachlorobutadiene & TIS & 7213 & & $\mathbf{A}$ & 2101 & 63 \\
\hline & & & & 1383 & 54 & Hexachlorobutadiene & WWR & 1098 & 39702 & $\mathbf{A}$ & 1394 & 58 \\
\hline \multirow[t]{2}{*}{ Fluorene } & BTM & 1150 & $34384 \mathrm{~A}$ & 1386 & 47 & & & & & & 1385 & 55 \\
\hline & & & & 1384 & 46 & & & & & & 1383 & 54 \\
\hline \multirow[t]{3}{*}{ Fluorene } & WWR & 1095 & 34381 A & 1394 & 58 & Hexachlorobutadiene & WWR & 1675 & 39702 & C & 1380 & 53 \\
\hline & & & & 1385 & S5 & & & & & & 1392 & 57 \\
\hline & & & & 1383 & 54 & Hexachlorobutadiene & WWR & 1676 & 39702 & B & 1390 & 56 \\
\hline \multirow[t]{3}{*}{ Fonofos } & WWR & 1336 & $82614 \mathrm{C}$ & 1474 & 59 & Hexachlorobutadiene & WWR & 5855 & 39702 & D & 2090 & 62 \\
\hline & & & & 1334 & 52 & Hexachlorocyclopentadiene & BTM & 1154 & 34389 & $\mathbf{A}$ & 1386 & 47 \\
\hline & & & & 1319 & 50 & & & & & & 1384 & 46 \\
\hline \multirow{2}{*}{ Fonofos } & WWR & 4022 & $04095 \mathrm{D}$ & 2001 & 60 & Hexachlorocyclopentadiene & TIS & 7015 & & $\mathbf{A}$ & 2101 & 63 \\
\hline & & 4222 & $04095 \mathrm{E}$ & 2010 & 60 & Hexachlorocyclopentadiene & WWR & 1099 & 34386 & $\mathbf{A}$ & 1385 & 55 \\
\hline GC/FID & BTM & 1236 & $99475 \mathrm{~A}$ & 1382 & 46 & & & & & & 1383 & 54 \\
\hline GC/FID & WWR & 1240 & 99478 A & 1381 & 53 & & & & & & 1394 & 58 \\
\hline $\mathrm{HCH}$ alpha & BTM & 5026 & -- B & 2501 & 47 & Hexachloroethane & BTM & 1155 & 34399 & $\mathbf{A}$ & 1386 & 47 \\
\hline $\mathrm{HCH}$ alpha & TIS & 7016 & $\cdots \mathrm{A}$ & 2101 & 63 & & & & & & 1384 & 46 \\
\hline \multirow[t]{2}{*}{$\mathrm{HCH}$ alpha } & WWR & 4023 & $34253 \mathrm{D}$ & 2001 & 60 & Hexachloroethane & WWR & 1100 & 34396 & A & 1394 & 58 \\
\hline & & 4223 & $34253 \mathrm{E}$ & 2010 & 60 & & & & & & 1385 & 55 \\
\hline $\mathrm{HCH}$ alpha d6-surrogate\% & BTM & 5032 & - B & 2501 & 47 & & & & & & 1383 & 54 \\
\hline \multirow[t]{2}{*}{ HCH alpha d6-surrogate\% } & WWR & 4024 & $91065 \mathrm{D}$ & 2001 & 60 & Hexazinone & WWR & 1466 & 30264 & $\mathbf{A}$ & 1389 & 56 \\
\hline & & 4224 & $91065 \mathrm{E}$ & 2010 & 60 & Indeno[1,2,3-cd]pyrene & BTM & 5241 & -- & B & 2502 & 48 \\
\hline $\mathrm{HCH}$ beta & BTM & 5027 & $-\mathbf{B}$ & 2501 & 47 & Indeno[1,2,3-cd]pyrene & BTM & 1156 & 34406 & $\mathbf{A}$ & 1386 & 47 \\
\hline $\mathrm{HCH}$ beta & TIS & 7017 & $-\mathrm{A}$ & 2101 & 63 & & & & & & 1384 & 46 \\
\hline $\mathrm{HCH}$ delta & TIS & 7018 & $-\mathbf{A}$ & 2101 & 63 & Indeno[ $[1,2,3-c d] p y r e n e$ & WWR & 1101 & 34403 & $\mathbf{A}$ & 1394 & 58 \\
\hline HCH gamma (Lindane) & BTM & 5022 & $--\mathbf{B}$ & 2501 & 47 & & & & & & 1385 & 55 \\
\hline $\mathrm{HCH}$ gamma & TIS & 7019 & $-\mathbf{A}$ & 2101 & 63 & & & & & & 1383 & 54 \\
\hline \multirow[t]{2}{*}{ HCH gamma (Lindane) } & WWR & 4025 & $39341 \mathrm{D}$ & 2001 & 60 & Isodrin & BTM & 5037 & & B & 2501 & 47 \\
\hline & & 4225 & $39341 \mathrm{E}$ & 2010 & 60 & Isophorone & BTM & 5242 & -. & B & 2502 & 48 \\
\hline \multirow[t]{2}{*}{ Heptachlor } & BTM & 368 & 39413 A & 1335 & 45 & Isophorone & BTM & 1157 & 34411 & $\mathbf{A}$ & 1386 & 47 \\
\hline & & & & 1325 & 45 & & & & & & 1384 & 46 \\
\hline Heptachlor & BTM & 5020 & $--\mathbf{B}$ & 2501 & 47 & Isophorone & WWR & 1102 & 34408 & $\mathbf{A}$ & 1394 & 58 \\
\hline Heptachlor & DIS & 470 & 39411 A & 1331 & 51 & & & & & & 1385 & 55 \\
\hline & & & & 1321 & 50 & & & & & & 1383 & 54 \\
\hline Heptachlor & TIS & 7012 & $--A$ & 2101 & 63 & Isopropylbenzene & WWR & 1659 & 77223 & $\mathbf{A}$ & 1380 & 53 \\
\hline Heptachlor & WWR & 357 & $39410 \mathrm{C}$ & 1474 & 59 & & & & & & 1392 & 57 \\
\hline & & & & 1334 & 52 & Isopropylbenzene & WWR & 1660 & 77223 & B & 1390 & 56 \\
\hline & & & & 1324 & 51 & Isoquinoline & BTM & 5261 & -. & B & 2502 & 48 \\
\hline Heptachlor & WWR & 744 & $39410 \mathrm{~B}$ & 1399 & 58 & Lindane & BTM & 370 & 39343 & $\mathbf{A}$ & 1335 & 45 \\
\hline & & & & 1398 & 58 & & & & & & 1325 & 45 \\
\hline Heptachlor & WWR & 1623 & $39410 \mathrm{D}$ & 1608 & 59 & Lindane & DIS & 472 & 39341 & $\mathbf{A}$ & 1331 & 51 \\
\hline Heptachlor epoxide & BTM & 369 & $39423 \mathrm{~A}$ & 1335 & 45 & & & & & & 1321 & 50 \\
\hline & & & & 1325 & 45 & Lindane & WWR & 359 & 39340 & C & 1474 & 59 \\
\hline Heptachlor epoxide & BTM & 5021 & $--\mathbf{B}$ & 2501 & 47 & & & & & & 1334 & 52 \\
\hline Heptachlor epoxide & DIS & 471 & $39421 \mathrm{~A}$ & 1331 & 51 & & & & & & 1324 & 51 \\
\hline & & & & 1321 & 50 & Lindane & WWR & 746 & 39340 & B & 1399 & 58 \\
\hline Heptachlor epoxide & TIS & 7013 & $-\mathbf{A}$ & 2101 & 63 & & & & & & 1398 & 58 \\
\hline Heptachlor epoxide & WWR & 358 & $39420 \mathrm{C}$ & 1474 & 59 & Lindane & WWR & 1621 & 39340 & D & 1608 & 59 \\
\hline & & & & 1334 & 52 & Linuron & WWR & 4026 & 82666 & D & 2001 & 60 \\
\hline & & & & 1324 & 51 & & & 4226 & 82666 & E & 2010 & 60 \\
\hline Heptachlor epoxide & WWR & 745 & $39420 \mathrm{~B}$ & 1399 & 58 & Linuron & WWR & 5432 & 38478 & $\mathbf{A}$ & 2050 & 61 \\
\hline & & & & 1398 & 58 & & & 5632 & 38478 & $\mathbf{A}$ & 2051 & 61 \\
\hline Heptachlor epoxide & WWR & 1625 & $39420 \mathrm{D}$ & 1608 & 59 & Lipids, percent & TIS & 7032 & -- & A & 2101 & 63 \\
\hline Hexachlorobenzene & BTM & 1152 & $39701 \mathrm{~A}$ & 1386 & 47 & MBAS & WWR & 96 & 38260 & $\mathbf{A}$ & -- & 43 \\
\hline
\end{tabular}




\begin{tabular}{|c|c|c|c|c|c|c|c|c|c|c|c|c|}
\hline Organic parameters--Continued & Phase & $\begin{array}{l}\text { Lab. } \\
\text { code }\end{array}$ & $\begin{array}{l}\text { Para- } \\
\text { meter }\end{array}$ & $\begin{array}{l}\text { Sche- } \\
\text { dule }\end{array}$ & Pg. & Organic parameters--Continued & Phase & $\begin{array}{l}\text { Lab. } \\
\text { code }\end{array}$ & $\begin{array}{l}\text { Para- } \\
\text { meter }\end{array}$ & & $\begin{array}{l}\text { Sche- } \\
\text { dule }\end{array}$ & Pg. \\
\hline \multirow[t]{2}{*}{$\overline{\mathrm{MCPA}}$} & WWR & $\overline{5433}$ & 38482 & 22050 & $\overline{61}$ & Methyllertbutylether & WWR & T652 & 78032 & $\overline{\mathrm{A}}$ & 1380 & $\overline{53}$ \\
\hline & & 5633 & 38482 & A 2051 & 61 & & & & & & 1392 & 57 \\
\hline \multirow[t]{2}{*}{ MCPB } & WWR & 5434 & 38487 & A 2050 & 61 & Methyltertbutylether & WWR & 1653 & 78032 & B & 1390 & 53 \\
\hline & & 5634 & 38487 & A 2051 & 61 & Metolachlor & DIS & 1593 & 39415 & $A$ & 1379 & 53 \\
\hline \multirow[t]{2}{*}{ Malathion } & BTM & 387 & 39531 & A 1335 & 45 & Metolachlor & WWR & 1334 & 82612 & C & 1389 & 56 \\
\hline & & & & 1320 & 45 & Metolachlor & WWR & 4029 & 39415 & D & 2001 & 60 \\
\hline \multirow[t]{2}{*}{ Malathion } & DIS & 425 & 39532 & A 1331 & 51 & & & 4229 & 39415 & $\mathrm{E}$ & 2010 & 60 \\
\hline & & & & 1316 & 50 & Metribuzin & DIS & 1594 & 82630 & A & 1379 & 53 \\
\hline Malathion & sUS & 419 & 39533 & A 1317 & 50 & Metribuzin & WWR & 1333 & 82611 & C & 1389 & 56 \\
\hline \multirow[t]{4}{*}{ Malathion } & WWR & 380 & 39530 & B 1474 & 59 & Metribuzin & WWR & 4030 & 82630 & D & 2001 & 60 \\
\hline & & & & 1399 & 58 & & & 4230 & 82630 & $\mathrm{E}$ & 2010 & 60 \\
\hline & & & & 1334 & 52 & Mirex & BTM & 545 & 39758 & A & 1335 & 45 \\
\hline & & & & 1319 & 50 & & & & & & 1325 & 45 \\
\hline \multirow[t]{2}{*}{ Malathion } & WWR & 4027 & 39532 & D 2001 & 60 & Mirex & BTM & 5023 & & B & 2501 & 47 \\
\hline & & 4227 & 39532 & E 2010 & 60 & Mirex & DIS & 542 & 39756 & A & 1331 & 51 \\
\hline Methane, bis(2-chloroethoxy)- & BTM & 5214 & -. & B 2502 & 48 & & & & & & 1321 & 50 \\
\hline Methane, bromo & WWR & 5804 & 34413 & C 2090 & 62 & Mirex & TIS & 7022 & & $A$ & 2101 & 63 \\
\hline Methane, bromochloro- & WWR & 5801 & 37297 & C 2090 & 62 & Mirex & WWR & 544 & 39755 & B & 1474 & 59 \\
\hline Methane, chloro & WWR & 5802 & 34418 & C 2090 & 62 & & & & & & 1399 & 58 \\
\hline Methane, dibromo- & WWR & 5824 & 30217 & C 2090 & 62 & & & & & & 1334 & 52 \\
\hline Methane, dibromochloro- & WWR & 5831 & 32105 & C 2090 & 62 & & & & & & 1398 & 58 \\
\hline Methane, dichloro & WWR & 5808 & 34423 & C 2090 & 62 & & & & & & 1324 & 51 \\
\hline Methane, dichlorobromo- & WWR & 5823 & 32101 & C 2090 & 62 & Moisture, percent & TIS & 7032 & & A & 2101 & 63 \\
\hline Methane, dichlorodifluoro- & WWR & 5801 & 34668 & C 2090 & 62 & Molinate & WWR & 4031 & 82671 & $\mathrm{D}$ & 2001 & 60 \\
\hline Methane, tetrachloro & WWR & 5817 & 32102 & C 2090 & 62 & & & 4231 & 82671 & $\mathrm{E}$ & 2010 & 60 \\
\hline Methane, tribromo (bromo) & WWR & 5838 & 32104 & C 2090 & 62 & n-Butylbenzene & WWR & 1671 & 77342 & $\mathbf{A}$ & 1380 & 53 \\
\hline Methane, trichloro (chloro) & WWR & 5815 & 32106 & C 2090 & 62 & & & & & & 1392 & 57 \\
\hline Methane, trichlorofluoro- & WWR & 5806 & 34488 & C 2090 & 62 & n-Butylbenzene & WWR & 1672 & 77342 & B & 1390 & 56 \\
\hline Methiocarb & WWR & 1449 & 30282 & A 1359 & 52 & n-Nitrosodi-n-propylamine & BTM & 1162 & 34431 & A & 1386 & 47 \\
\hline \multirow[t]{2}{*}{ Methiocarb } & WWR & 5436 & 38501 & A 2050 & 61 & & & & & & 1384 & 46 \\
\hline & & 5636 & 38501 & A 2051 & 61 & n-Nitrosodi-n-propylamine & WWR & 1107 & 34428 & A & 1394 & 58 \\
\hline \multirow[t]{2}{*}{ Methomyl } & WWR & 5437 & - & A 2050 & 61 & & & & & & 1385 & 55 \\
\hline & & 5637 & -- & A 2051 & 61 & & & & & & 1383 & 54 \\
\hline Methomyl & WWR & 638 & 39051 & A 1359 & 52 & n-Nitrosodimethylatnine & BTM & 1160 & 34441 & $\mathbf{A}$ & 1386 & 47 \\
\hline \multirow[t]{2}{*}{ Methoxychlor } & BTM & 401 & 39481 & A 1335 & 45 & & & & & & 1384 & 48 \\
\hline & & & & 1325 & 45 & n-Nitrosodimethylamine & WWR & 1105 & 34438 & $\mathbf{A}$ & 1394 & 58 \\
\hline \multirow[t]{2}{*}{ Methoxychlor } & DIS & 476 & 82350 & A 1331 & 51 & & & & & & 1385 & 55 \\
\hline & & & & 1321 & 50 & & & & & & 1383 & 54 \\
\hline \multirow[t]{5}{*}{ Methoxychlor } & WWR & 400 & 39480 & B 1474 & 59 & n-Nitrosodiphenylamine & BTM & 1161 & 34436 & A & 1386 & 47 \\
\hline & & & & 1399 & 58 & & & & & & 1384 & 46 \\
\hline & & & & 1334 & 52 & n-Nitrosodiphenylamine & WWR & 1106 & 34433 & A & 1394 & 58 \\
\hline & & & & 1398 & 58 & & & & & & 1385 & 55 \\
\hline & & & & 1324 & 51 & & & & & & 1383 & 54 \\
\hline Methoxychlor, o,p'- & BTM & 5042 & -- & B 2501 & 47 & n-propylbenzene & WWR & 1661 & 77224 & A & 1380 & 53 \\
\hline Methoxychlor, o,p & TIS & 7020 & -- & A 2101 & 63 & & & & & & 1392 & 57 \\
\hline Methoxychlor, p,p'- & BTM & 5044 & -- & B 2501 & 47 & n-Propylbenzene & WWR & 1662 & 77224 & B & 1390 & 56 \\
\hline Methoxychlor, p,p' & TIS & 7021 & - & A 2101 & 63 & Naphthalene & BTM & 5246 & -- & B & 2502 & 48 \\
\hline \multirow[t]{2}{*}{ Methyl parathion } & BTM & 388 & 39601 & A 1335 & 45 & Naphthalene & BTM & 1158 & 34445 & A & 1386 & 47 \\
\hline & & & & 1320 & 45 & & & & & & 1384 & 46 \\
\hline Methyl parathion & DIS & 426 & 39602 & A 1331 & 51 & Naphthalene & WWR & 1103 & 34696 & A & 1385 & 55 \\
\hline & & & & 1316 & 50 & & & & 34696 & A & 1383 & 54 \\
\hline Methyl parathion & sus & 420 & 39603 & A 1317 & 50 & & & & 34696 & $\mathbf{A}$ & 1394 & 58 \\
\hline Methyl parathion & WWR & 381 & 39600 & B 1474 & 59 & Naphthalene & WWR & 1677 & 34696 & $\mathrm{C}$ & 1380 & 53 \\
\hline & & & & 1399 & 58 & & & & & & 1392 & 57 \\
\hline & & & & 1334 & 52 & Naphthalene & WWR & 1678 & 34696 & B & 1390 & 56 \\
\hline & & & & 1319 & 50 & Naphthalene & WWR & 5856 & 34696 & $\mathrm{D}$ & 2090 & 62 \\
\hline Methylene chloride & WWR & 1029 & 34423 & A 1390 & 56 & Naphthalene, 1,2-dimethyl- & BTM & 5267 & -- & B & 2502 & 48 \\
\hline Methylene chloride & WWR & 1305 & 34423 & B 1307 & 49 & Naphthalene, 1,6-dimethyl & BTM & 5266 & -- & B & 2502 & 48 \\
\hline & & & & 1380 & 53 & Naphthalene, 2,3,6-trimethyl- & BTM & 5270 & -- & B & 2502 & 48 \\
\hline & & & & 1387 & 55 & Naphthalene, 2,6-dimethyl- & BTM & 5265 & - & B & 2502 & 48 \\
\hline & & & & 1392 & 57 & Naphthalene, 2-chloro & BTM & 5207 & -- & B & 2502 & 48 \\
\hline
\end{tabular}




\begin{tabular}{|c|c|c|c|c|c|c|c|c|c|c|c|}
\hline Organic parameters--Continued & Phase & $\begin{array}{l}\text { Lab. } \\
\text { code }\end{array}$ & $\begin{array}{l}\text { Para- } \\
\text { meter }\end{array}$ & $\begin{array}{l}\text { Sche- } \\
\text { dule }\end{array}$ & Pg. & Organic parameters--Continued & Phase & $\begin{array}{l}\text { Lab. } \\
\text { code }\end{array}$ & $\begin{array}{l}\text { Para- } \\
\text { meter }\end{array}$ & $\begin{array}{l}\text { Sche- } \\
\text { dule }\end{array}$ & Pg. \\
\hline Naphthalene, 2-ethyl- & BTM & 5264 & $-\mathrm{B}$ & 2502 & $\overline{48}$ & Parathion, methyl- & WWR & 4028 & 826710 & 2001 & $\overline{60}$ \\
\hline \multirow[t]{2}{*}{ Napropamide } & WWR & 4033 & $82667 \mathrm{D}$ & 2001 & 60 & & & 4228 & $82667 \mathrm{E}$ & 2010 & 60 \\
\hline & & 4233 & $82667 \mathrm{E}$ & 2010 & 60 & Pebulate & WWR & 4034 & 82669 D & 2001 & 60 \\
\hline \multirow[t]{2}{*}{ Neburon } & WWR & 5403 & $-A$ & 2050 & 61 & & & 4234 & 82669 E & 2010 & 60 \\
\hline & & 5603 & $\cdots \mathbf{A}$ & 2051 & 61 & Pendimethal in & WWR & 4035 & $82683 \mathrm{D}$ & 2001 & 60 \\
\hline \multirow[t]{2}{*}{ Nitrobenzene } & BTM & 1159 & $34450 \mathrm{~A}$ & 1386 & 47 & & & 4235 & $82683 \mathrm{E}$ & 2010 & 60 \\
\hline & & & & 1384 & 46 & Pentachloroanisole & BTM & 5274 & $--\mathrm{B}$ & 2502 & 48 \\
\hline \multirow[t]{3}{*}{ Nitrobenzene } & WWR & 1104 & 34447 A & 1385 & 55 & Pentachloroanisole & BTM & 5033 & $--\mathbf{B}$ & 2501 & 47 \\
\hline & & & & 1383 & 54 & Pentachloroanisole & TIS & 7027 & $--A$ & 2101 & 63 \\
\hline & & & & 1394 & 58 & Pentachlorophenol & BTM & 1052 & $39061 \mathrm{~A}$ & 1386 & 47 \\
\hline Nonachlor, cis & BTM & 5041 & $-\mathbf{B}$ & 2501 & 47 & & & & & 1384 & 46 \\
\hline Nonachlor, cis & TIS & 7023 & $-A$ & 2101 & 63 & Pentachlorophenol & WWR & 1064 & 39032 A & 1385 & 55 \\
\hline Nonachlor, trans & BTM & 5034 & -- B & 2501 & 47 & & & & & 1393 & 57 \\
\hline Nonachlor, trans & TIS & 7024 & - A & 2101 & 63 & & & & & 1383 & 54 \\
\hline \multirow[t]{2}{*}{ Norflurazon } & WWR & 5439 & $-\mathbf{A}$ & 2050 & 61 & Permethrin cis isomer & BTM & 5055 & - B & 2501 & 47 \\
\hline & & 5639 & $-A$ & 2051 & 61 & Permethrin, cis-isomer & WWR & 4036 & 82687 D & 2001 & 60 \\
\hline Octachlorobiphenyl surrogate \% & BTM & 5048 & -- $\mathbf{B}$ & 2501 & 47 & & & 4236 & 82687 E & 2010 & 60 \\
\hline Oil and grease & BTM & 531 & $557 \mathrm{~A}$ & -- & 43 & Permethrin trans isomer & BTM & 5056 & $-\mathrm{B}$ & 2501 & 47 \\
\hline Oil and grease & Total & 127 & $556 \mathrm{~A}$ & -- & 43 & Perthane & BTM & 342 & 81886 A & 1335 & 45 \\
\hline \multirow[t]{2}{*}{ Oryzalin (Surflan } & WWR & 5440 & $-\mathbf{A}$ & 2050 & 61 & & & & & 1325 & 45 \\
\hline & & 5640 & $-\mathbf{A}$ & 2051 & 61 & Perthane & DIS & 344 & 82348 A & 1331 & 51 \\
\hline \multirow[t]{2}{*}{ Oxamyl } & WWR & 5441 & $38866 \mathrm{~A}$ & 2050 & 61 & & & & & 1321 & 59 \\
\hline & & 5641 & $38866 \mathrm{~A}$ & 2051 & 61 & Perthane & WWR & 348 & 39034 A & 1474 & 62 \\
\hline Oxamyl & WWR & 1335 & $82613 \mathrm{C}$ & 1359 & 52 & & & & & 1399 & 58 \\
\hline Oxychlordane & BTM & 5038 & $--\mathbf{B}$ & 2501 & 47 & & & & & 1334 & 52 \\
\hline Oxychlordane & TIS & 7025 & $-\mathrm{A}$ & 2101 & 63 & & & & & 1398 & 58 \\
\hline \multirow[t]{2}{*}{ PCB's, gross } & BTM & 394 & 39519 A & 1335 & 45 & & & & & 1324 & 51 \\
\hline & & & & 1325 & 45 & Phenanthrene & BTM & 5248 & $-\mathbf{B}$ & 2502 & 48 \\
\hline \multirow[t]{2}{*}{ PCB's, gross } & DIS & 474 & 39517 A & 1331 & 51 & Phenanthrene & BTM & 1163 & 34464 A & 1386 & 47 \\
\hline & & & & 1321 & 50 & & & & & 1384 & 46 \\
\hline \multirow[t]{5}{*}{ PCB's, gross } & WWR & 392 & $39516 \mathrm{~B}$ & 1474 & 59 & Phenanthrene & WWR & 1108 & 34461 A & 1383 & 54 \\
\hline & & & & 1399 & 58 & & & & & 1394 & 58 \\
\hline & & & & 1334 & 52 & & & & & 1385 & 55 \\
\hline & & & & 1398 & 58 & Phenanthrene, 1-methyl & BTM & 5282 & $-\mathrm{B}$ & 2502 & 48 \\
\hline & & & & 1324 & 51 & Phenanthrene, 4,5-methylene- & BTM & 5281 & - B & 2502 & 48 \\
\hline \multirow[t]{2}{*}{ PCN's, gross } & BTM & 395 & 39251 A & 1335 & 45 & Phenanthridine & BTM & 5277 & - B & 2502 & 48 \\
\hline & & & & 1325 & 45 & Phenol & BTM & 5249 & $-\mathbf{B}$ & 2502 & 48 \\
\hline \multirow[t]{2}{*}{ PCN's, gross } & DIS & 475 & $82360 \mathrm{~A}$ & 1331 & 51 & Phenol & BTM & 1053 & 34695 A & 1386 & 47 \\
\hline & & & & 1321 & 50 & & & & & 1384 & 40 \\
\hline PCN's, gross & WWR & 393 & $39250 \mathrm{~B}$ & 1474 & 59 & Phenol & WWR & 1065 & 34694 A & 1383 & 54 \\
\hline & & & & 1399 & 58 & & & & & 1385 & 55 \\
\hline & & & & 1334 & 52 & & & & & 1393 & 57 \\
\hline & & & & 1398 & 58 & Phenol, 2,3,5,6-tetramethyl- & BTM & 5263 & $-\mathrm{B}$ & 2502 & 48 \\
\hline & & & & 1324 & 51 & Phenol, 2,3,6-trichloro- & BTM & 5204 & $-\mathbf{B}$ & 2502 & 48 \\
\hline PCBs, total & BTM & 5024 & $-\mathbf{B}$ & 2501 & 47 & Phenol, 2,4,6-trimethyl- & BTM & 5259 & $--\mathbf{B}$ & 2502 & 48 \\
\hline PCBs, total & TIS & 7029 & $-\mathbf{A}$ & 2101 & 63 & Phenol, 2,4-dichloro- & BTM & 5257 & - B & 2502 & 48 \\
\hline p-Cresol & BTM & 5254 & $-\mathrm{B}$ & 2502 & 48 & Phenol, 2,4-dinitro- & BTM & 5268 & - B & 2502 & 48 \\
\hline p-isopropyltoluene & WWR & 1669 & 77356 A & 1380 & 53 & Phenol, 2-methyl-4,6-dinitro- & BTM & 5271 & $-\mathrm{B}$ & 2502 & 48 \\
\hline & & & & 1392 & 57 & Phenol, 2-nitro- & BTM & 5255 & $-\mathbf{B}$ & 2502 & 48 \\
\hline p-isopropyltoluene & WWR & 1670 & $77356 \mathrm{~B}$ & 1390 & 56 & Phenol, 3,5-dimethyl- & BTM & 5258 & $-\mathbf{B}$ & 2502 & 48 \\
\hline Parathion & BTM & 390 & $39541 \mathrm{~A}$ & 1335 & 45 & Phenol, 4-chloro-3-methyl- & BTM & 5262 & $-\mathbf{B}$ & 2502 & 48 \\
\hline & & & & 1320 & 45 & Phenol, 4-nitro- & BTM & 5269 & $-\mathbf{B}$ & 2502 & 48 \\
\hline Parathion & DIS & 427 & 39542 A & 1331 & 51 & Phenol, C8-alkyl- & BTM & 5256 & - B & 2502 & 48 \\
\hline & & & & 1316 & 50 & Phenol, pentachloro & BTM & 5227 & - B & 2502 & 48 \\
\hline Parathion & SUS & 421 & $39543 \mathrm{~A}$ & 1317 & 50 & Phenols, total, colorimetric & Total & 52 & $32730 \mathrm{~A}$ & -- & 43 \\
\hline Parathion & WWR & 383 & $39540 \mathrm{~B}$ & 1474 & 59 & Phorate & WWR & 593 & $39023 \mathrm{~A}$ & 1474 & 59 \\
\hline & & & & 1399 & 58 & & & & & 1334 & 52 \\
\hline & & & & 1334 & 52 & & & & & 1319 & 50 \\
\hline & & & & 1319 & 50 & Phorate & WWR & 4037 & 82664 D & 2001 & 60 \\
\hline Parathion, ethyl- & WWR & 4033 & $39542 \mathrm{D}$ & 2001 & 60 & & & 4237 & $82664 \mathrm{E}$ & 2010 & 60 \\
\hline & & 4233 & $39542 \mathrm{D}$ & 2010 & 60 & Phthalate, bis(2-ethylhexyl)- & BTM & 5223 & $-\mathbf{B}$ & 2502 & 48 \\
\hline
\end{tabular}


INDEX

Constituents in alphabetical order--Continued

\begin{tabular}{|c|c|c|c|c|c|c|c|c|c|c|c|}
\hline Organic parameters--Continued & Phase & $\begin{array}{l}\text { Lab. } \\
\text { code }\end{array}$ & $\begin{array}{l}\text { Para- } \\
\text { meter }\end{array}$ & $\begin{array}{l}\text { Sche- } \\
\text { dule }\end{array}$ & Pg. & Organic parameters--Continued & Phase & $\begin{array}{l}\text { Lab. } \\
\text { code }\end{array}$ & $\begin{array}{l}\text { Para- } \\
\text { meter }\end{array}$ & $\begin{array}{l}\text { Sche- } \\
\text { dule }\end{array}$ & Pg. \\
\hline Phthalae, butybenzyl- & $\overline{\text { BTM }}$ & 5224 & $-\bar{B}$ & 2502 & $\overline{48}$ & Simazine & $\overline{\text { WWR }}$ & 4043 & $04035 \mathrm{D}$ & 2001 & $\overline{60}$ \\
\hline Phthalate, di-n-butyl & BTM & 5235 & $--\mathbf{B}$ & 2502 & 48 & & & 4243 & $04035 \mathrm{E}$ & 2010 & 60 \\
\hline Phthalate, di-n-octyl- & BTM & 5239 & -- B & 2502 & 48 & Simetryn & WWR & 720 & $39054 \mathrm{~A}$ & 1389 & 56 \\
\hline Phthalate, diethyl & BTM & 5237 & $--\mathbf{B}$ & 2502 & 48 & Styrene & WWR & 1325 & $77128 \mathrm{~A}$ & 1390 & 56 \\
\hline Phthalate, dimethyl- & BTM & 5238 & $\ldots \mathbf{B}$ & 2502 & 48 & Styrene & WWR & 1328 & $77128 \mathrm{~B}$ & 1307 & 49 \\
\hline Picloram & BIM & 750 & $38930 \mathrm{~A}$ & 80 & 45 & & & & & 1380 & 53 \\
\hline Picloram & WWR & 748 & $39720 \mathrm{~A}$ & 79 & 49 & & & & & 1392 & 57 \\
\hline \multirow[t]{2}{*}{ Picloram } & WWR & 5442 & $-A$ & 2050 & 61 & Styrene & WWR & 5837 & $77128 \mathrm{C}$ & 2090 & 62 \\
\hline & & 5642 & $-A$ & 2051 & 61 & Tannin and lignin & WWR & 138 & $32240 \mathrm{~A}$ & -- & 43 \\
\hline Prometon & DIS & 1597 & $04037 \mathrm{~A}$ & 1379 & 53 & Tebuthiuron & WWR & 4045 & $82670 \mathrm{D}$ & 2001 & 60 \\
\hline Prometon & WWR & 718 & $39056 \mathrm{~A}$ & 1389 & 56 & & & 4245 & $82670 \mathrm{E}$ & 2010 & 60 \\
\hline \multirow[t]{2}{*}{ Prometon } & WWR & 4038 & $82676 \mathrm{D}$ & 2001 & 60 & Terbacil & WWR & 1462 & $30311 \mathrm{~A}$ & 1389 & 56 \\
\hline & & 4239 & $82676 \mathrm{E}$ & 2010 & 60 & Terbacil & WWR & 4046 & $82665 \mathrm{D}$ & 2001 & 60 \\
\hline Prometryn & DIS & 1598 & $04036 \mathrm{~A}$ & 1379 & 53 & & & 4246 & $82665 \mathrm{E}$ & 2010 & 60 \\
\hline Prometryn & WWR & 631 & 39057 A & 1389 & 56 & Terbufos & WWR & 4047 & $82675 \mathrm{D}$ & 2001 & 60 \\
\hline \multirow[t]{2}{*}{ Pronamide } & WWR & 4038 & $82676 \mathrm{D}$ & 2001 & 60 & & & 4247 & $82675 \mathrm{E}$ & 2010 & 60 \\
\hline & & 4238 & $82676 \mathrm{E}$ & 2010 & 60 & Terphenyl, d14-surrogate \% & BTM & 5286 & $--\mathbf{B}$ & 2502 & 48 \\
\hline Propachlor & WWR & 1471 & $30295 \mathrm{~A}$ & 1389 & 56 & Tert-butylbenzene & WWR & 4047 & $91064 \mathrm{D}$ & 2001 & 60 \\
\hline \multirow{2}{*}{ Propachlor } & WWR & 4040 & $04024 \mathrm{D}$ & 2001 & 60 & & & 4247 & $91064 \mathrm{E}$ & 2010 & 60 \\
\hline & & 4240 & $04024 \mathrm{E}$ & 2010 & 60 & Tert-butylbenzene & WWR & 1663 & $77353 \mathrm{~A}$ & 1380 & 53 \\
\hline Propane, 1,2-dibromo-3-chloro & WWR & 5842 & $77443 \mathrm{C}$ & 2090 & 62 & & & & & 1392 & 57 \\
\hline Propane, 1,2-dichloro- & WWR & 5822 & $34541 \mathrm{C}$ & 2090 & 62 & Tert-butylbenzene & WWR & 1664 & $77353 \mathrm{~B}$ & 1390 & 56 \\
\hline Propane, 1,2,3-trichloro & WWR & 5842 & $77443 \mathrm{C}$ & 2090 & 62 & Tetrachloroethene & WWR & 1307 & $34475 \mathrm{~B}$ & 1307 & 49 \\
\hline Propane, 1,3-dichloro- & WWR & 5830 & $77173 \mathrm{C}$ & 2090 & 62 & & & & & 1380 & 53 \\
\hline Propane, 2,2-dichloro & WWR & 5812 & $77170 \mathrm{C}$ & 2090 & 62 & & & & & 1392 & 57 \\
\hline \multirow[t]{2}{*}{ Propanil } & WWR & 4041 & $82679 \mathrm{D}$ & 2001 & 60 & Tetrachloroethylene & WWR & 1031 & $34475 \mathrm{~A}$ & 1390 & 56 \\
\hline & & 4241 & $82679 \mathrm{E}$ & 2010 & 60 & Thiobencarb & WWR & 4044 & $82681 \mathrm{D}$ & 2001 & 60 \\
\hline \multirow{2}{*}{ Propargite } & WWR & 4042 & $82685 \mathrm{D}$ & 2001 & 60 & & & 4244 & $82681 \mathrm{E}$ & 2010 & 60 \\
\hline & & 4242 & $82685 \mathrm{E}$ & 2010 & 60 & Toluene & WWR & 1032 & $34010 \mathrm{~A}$ & 1390 & 56 \\
\hline Propazine & DIS & 1595 & $38535 \mathrm{~A}$ & 1379 & 53 & Toluene & WWR & 1308 & $34010 \mathrm{~B}$ & 1307 & 49 \\
\hline Propazine & WWR & 844 & 39024 A & 1389 & 56 & & & & & 1378 & 52 \\
\hline Propene, 1,1-dichloro & WWR & 5818 & $77168 \mathrm{C}$ & 2090 & 62 & Toluene & WWR & 1308 & $34010 \mathrm{~B}$ & 1380 & 53 \\
\hline Propene, cis-1,3-dichloro & WWR & 5825 & $34704 \mathrm{C}$ & 2090 & 62 & & & & & 1387 & 55 \\
\hline Propene, trans-1,3-dichloro & WWR & 5827 & $34699 \mathrm{C}$ & 2090 & 62 & & & & & 1392 & 57 \\
\hline Propham & WWR & 637 & $39052 \mathrm{~A}$ & 1359 & 52 & Toluene, 2,4-dinitro- & BTM & 5203 & - B & 2502 & 48 \\
\hline \multirow[t]{2}{*}{ Propham (IPC) } & WWR & 5443 & $--\mathrm{A}$ & 2050 & 61 & Toluene, 2,6-dinitro- & BTM & 5205 & - B & 2502 & 48 \\
\hline & & 5643 & $-A$ & 2051 & 61 & Toxaphene & BTM & 371 & $39403 \mathrm{~A}$ & 1335 & 45 \\
\hline \multirow[t]{2}{*}{ Propoxur } & WWR & 5450 & 38538 A & 2050 & 61 & & & & & 1325 & 45 \\
\hline & & 5650 & $38538 \mathrm{~A}$ & 2051 & 61 & Toxaphene & BTM & 5025 & -- B & 2501 & 47 \\
\hline Propoxur & WWR & 1448 & $30296 \mathrm{~A}$ & 1359 & 52 & Toxaphene & DIS & 473 & $39401 \mathrm{~A}$ & 1331 & 51 \\
\hline Pyrene & BTM & 5252 & $--\mathrm{B}$ & 2502 & 48 & & & & & 1321 & 50 \\
\hline \multirow[t]{2}{*}{ Pyrene } & BTM & 1164 & 34472 A & 1386 & 47 & Toxaphene & TIS & 7028 & $-\mathrm{A}$ & 2101 & 63 \\
\hline & & & & 1384 & 46 & Toxaphene & WWR & 360 & $39400 \mathrm{~B}$ & 1474 & 59 \\
\hline \multirow[t]{3}{*}{ Pyrene } & WWR & 1109 & 34469 A & 1385 & 55 & & & & & 1399 & 58 \\
\hline & & & & 1383 & 54 & & & & & 1334 & 52 \\
\hline & & & & 1394 & 58 & & & & & 1398 & 58 \\
\hline Pyrene, 1-methyl- & BTM & 5284 & $-\mathrm{B}$ & 2502 & 48 & & & & & 1324 & 51 \\
\hline Quinoline & BTM & 5260 & -- B & 2502 & 48 & Trans 1,2-dichloroethene & WWR & 1024 & $34546 \mathrm{~A}$ & 1390 & 56 \\
\hline \multirow[t]{2}{*}{ Sec-butylbenzene } & WWR & 1667 & $77350 \mathrm{~A}$ & 1380 & 53 & Trans 1,2-dichloroethene & WWR & 1300 & $34546 \mathrm{~B}$ & 1380 & 53 \\
\hline & & & & 1392 & 57 & & & & & 1392 & 57 \\
\hline Sec-butylbenzene & WWR & 668 & $77350 \mathrm{~B}$ & 1390 & 56 & & & & & 1307 & 49 \\
\hline \multirow[t]{2}{*}{ Silvex } & BTM & 377 & $39761 \mathrm{~A}$ & 1305 & 45 & Trans-1,3-dichloropropene & WWR & 1324 & 34699 A & 1390 & 56 \\
\hline & & & & 80 & 45 & Trans-1,3-dichloropropene & WWR & & $34699 \mathrm{~B}$ & 1380 & 53 \\
\hline Silvex & DIS & 479 & $39762 \mathrm{~A}$ & 1301 & 49 & & & 1327 & & & \\
\hline Silvex & WWR & 374 & $39760 \mathrm{~B}$ & 1474 & 59 & & & & & 1392 & 57 \\
\hline & & & & 79 & 49 & Triallate & WWR & 4049 & $82678 \mathrm{D}$ & 2001 & 60 \\
\hline & & & & 1304 & 49 & & & 4249 & $82678 \mathrm{E}$ & 2010 & 60 \\
\hline Silvex $(2,4,5-T P)$ & WWR & 5444 & $39762 \mathrm{~B}$ & 2050 & 61 & Trichloroethene & WWR & 1311 & $39180 \mathrm{~B}$ & 1307 & 49 \\
\hline & & 5644 & $39762 \mathrm{~B}$ & 2051 & 61 & & & & & 1380 & 53 \\
\hline Simazine & DIS & 1596 & $04035 \mathrm{~A}$ & 1379 & 53 & & & & & 1387 & 55 \\
\hline Simazine & WWR & 719 & $39055 \mathrm{~A}$ & 1389 & 56 & & & & & 1392 & 57 \\
\hline
\end{tabular}


INDEX

Constituents in alphabetical order--Continued

\begin{tabular}{|c|c|c|c|c|c|}
\hline Organic parameters--Continued & Phase & $\begin{array}{l}\text { Lab. } \\
\text { code }\end{array}$ & $\begin{array}{l}\text { Para- } \\
\text { meter }\end{array}$ & $\begin{array}{l}\text { Sche- } \\
\text { dule }\end{array}$ & Pg. \\
\hline Trichoroethylene & WWR & 1035 & 39180 & 1390 & 56 \\
\hline Trichlorofluoromethane & WWR & 1036 & 34488 & 1390 & 56 \\
\hline \multirow[t]{2}{*}{ Trichlorofluoromethane } & WWR & 1312 & 34488 & 1307 & 49 \\
\hline & & & & 1380 & 53 \\
\hline Trichlorofluoromethane & WWR & 1312 & 34488 & 1392 & 57 \\
\hline \multirow[t]{2}{*}{ Triclopyr } & WWR & 5446 & - & 2050 & 61 \\
\hline & & 5646 & & 2051 & 61 \\
\hline Trifluralin & WWR & 1332 & 39030 & 1389 & 56 \\
\hline \multirow{2}{*}{ Trifluralin } & WWR & 4050 & 82661 & 2001 & 60 \\
\hline & & 4250 & 82661 & 2010 & 60 \\
\hline \multirow[t]{2}{*}{ Trithion } & BTM & 391 & 39787 & 1335 & 45 \\
\hline & & & & 1320 & 45 \\
\hline \multirow[t]{2}{*}{ Trithion } & DIS & 428 & 82342 & 1331 & 51 \\
\hline & & & & 1316 & 50 \\
\hline Trithion & sUS & 422 & 82343 & 1317 & 50 \\
\hline \multirow[t]{4}{*}{ Trithion } & WWR & 394 & 39786 & 1474 & 59 \\
\hline & & & & 1399 & 58 \\
\hline & & & & 1334 & 52 \\
\hline & & & & 1319 & so \\
\hline Vemolate & WWR & 1467 & 30324 & 1389 & 56 \\
\hline Vinyl chloride & WWR & 1037 & 39175 & 1390 & 56 \\
\hline \multirow[t]{3}{*}{ Vinyl chloride } & WWR & 1313 & 39175 & 1307 & 49 \\
\hline & & & & 1380 & 53 \\
\hline & & & & 1392 & 57 \\
\hline \multirow[t]{2}{*}{ Xylenes, meta and para } & WWR & 1712 & 85795 & 1307 & 49 \\
\hline & & & & 1378 & 53 \\
\hline \multirow[t]{2}{*}{ Xylenes, ortho } & WWR & 1709 & 77135 & 1307 & 49 \\
\hline & & & & 1378 & 53 \\
\hline Xylenes, total $(o, m, p)$ & WWR & 1329 & 81551 & 1390 & 56 \\
\hline \multirow[t]{5}{*}{ Xylenes, total $(o, m, p)$} & WWR & 1330 & 81551 & 1307 & 49 \\
\hline & & & & 1378 & 53 \\
\hline & & & & 1380 & 53 \\
\hline & & & & 1387 & 55 \\
\hline & & & & 1392 & 57 \\
\hline
\end{tabular}




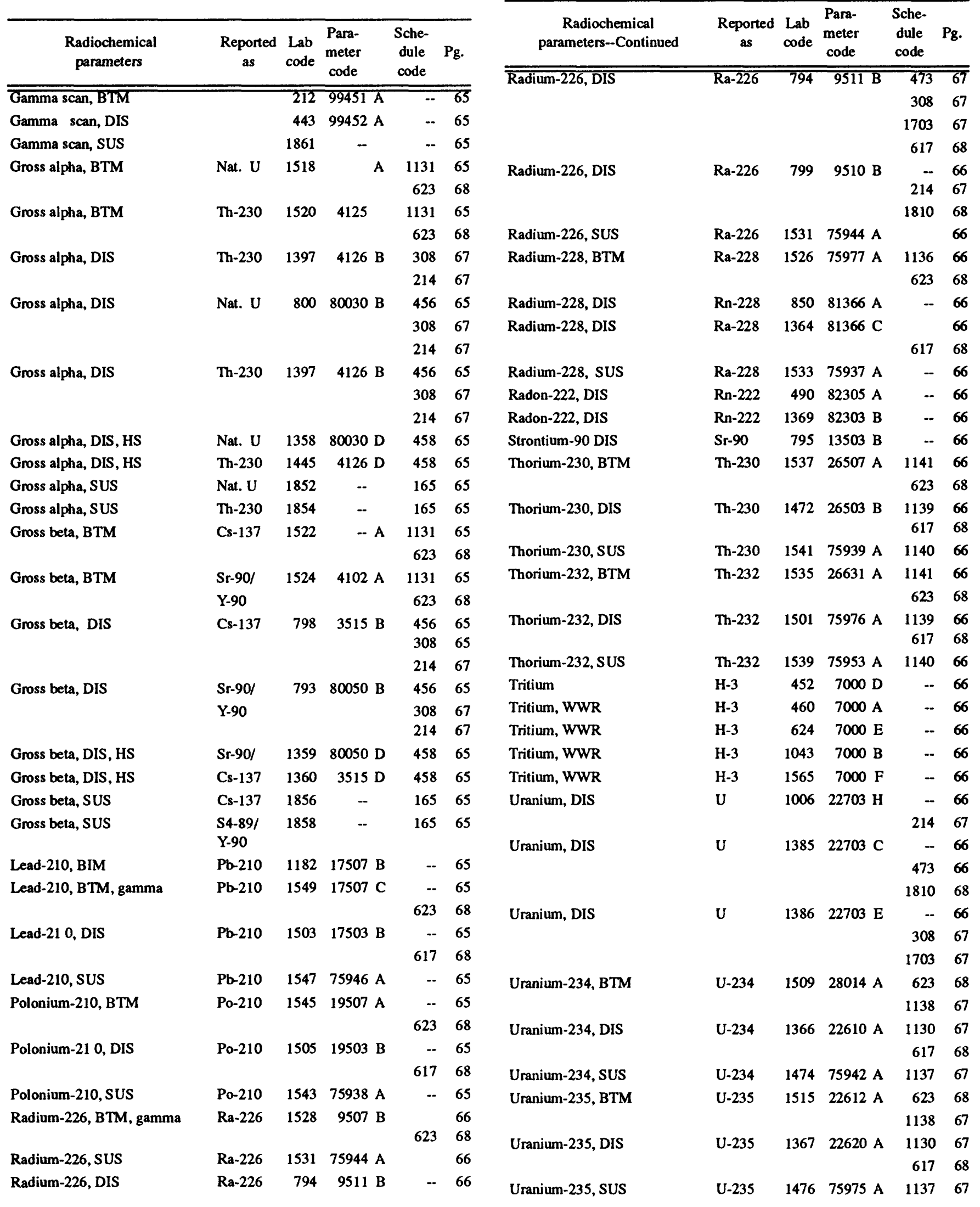




\begin{tabular}{|c|c|c|c|c|c|}
\hline $\begin{array}{c}\text { Radiochemical } \\
\text { parameters--Continued }\end{array}$ & $\begin{array}{l}\text { Reported } \\
\text { as }\end{array}$ & $\begin{array}{l}\text { Lab } \\
\text { code }\end{array}$ & $\begin{array}{l}\text { Para- } \\
\text { meter } \\
\text { code }\end{array}$ & $\begin{array}{l}\text { Sche- } \\
\text { dule } \\
\text { code }\end{array}$ & Pg. \\
\hline Uranium-238, BTM & $\overline{U-238}$ & 1511 & $\overline{28016 \mathrm{~A}}$ & $\begin{array}{r}1138 \\
623\end{array}$ & $\begin{array}{l}67 \\
68\end{array}$ \\
\hline Uranium-238, DIS & U-238 & 1368 & 22603 A & $\begin{array}{r}1130 \\
617\end{array}$ & $\begin{array}{l}67 \\
68\end{array}$ \\
\hline Uranium-238, SUS & $\mathrm{U}-238$ & 1507 & $75940 \mathrm{~A}$ & 1137 & 67 \\
\hline Stable Isotopes & & & & & \\
\hline $\begin{array}{l}\text { Carbon-13/Carbon-12, } \\
\text { whole water }\end{array}$ & & 440 & 82081 A & -- & 69 \\
\hline $\mathrm{C}-13 / \mathrm{C}-12$ and $0-18 / 0-16$ & & 1243 & $99481 \mathrm{~A}$ & & 69 \\
\hline $\begin{array}{l}\text { Carbon-13/Carbon-12, } \\
\text { carbonate rock }\end{array}$ & & 1135 & 82339 A & & 69 \\
\hline $\begin{array}{l}\text { Carbon-13/Carbon-12, } \\
\text { gaseous } \mathrm{CO}_{2}\end{array}$ & & 1244. & -..- &.- & 69 \\
\hline $\begin{array}{l}\text { Carbon-13/Carbon-12, } \\
\text { organic soil or rock material }\end{array}$ & & 1205 & -- -- & -- & 69 \\
\hline Carbon-14 (field precipitated) & & 1199 & 82172 B & & 69 \\
\hline $\begin{array}{l}\text { Carbon-14, aqueous } \\
\text { percent modem }\end{array}$ & & 1198 & $82172 \mathrm{~A}$ & & 69 \\
\hline Carbon-14, percent error & & 640 & $99454 \mathrm{~A}$ & -- & 69 \\
\hline Deuterium/protium, aq. & & 1574 & 82082 B & -- & 69 \\
\hline $\begin{array}{l}\text { Nitrogen-15/Nitrogen-14 ratio, } \\
\text { solid as ammonia }\end{array}$ & & 1719 & $-\mathrm{A}$ & - & 69 \\
\hline $\begin{array}{l}\text { Nitrogen-15/Nitrogen-14 ratio, } \\
\text { DIS as ammonia }\end{array}$ & & 1717 & $-\mathrm{A}$ & - & 69 \\
\hline $\begin{array}{l}\text { Nitrogen-15/Nitrogen-14 ration, } \\
\text { DIS as nitrate \& ammonia }\end{array}$ & & 1921 & $-A$ & -- & 69 \\
\hline $\begin{array}{l}\text { Nitrogen- } 15 / \text { Nitrogen-14 ratio, } \\
\text { DIS as nitrate }\end{array}$ & & 1718 & $-\mathbf{A}$ & -- & 69 \\
\hline $\begin{array}{l}\text { Nitrogen- } 15 / \text { Nitrogen-14 ratio, } \\
\text { solid as nitrate }\end{array}$ & & 1720 & $-\mathrm{A}$ &.- & 69 \\
\hline Oxygen-18/Oxygen-16, rock & & 1137 & $82337 \mathrm{~A}$ & -- & 69 \\
\hline Oxygen-18/Oxygen-16 & & 489 & 82085 A & & 69 \\
\hline ratio, aqueous & & & & 1142 & 70 \\
\hline $\begin{array}{l}\text { Sulfur-34/Sulfur-32, as sulfate } \\
\text { (high concentration), aqueous }\end{array}$ & & 1951 & -- & -- & 70 \\
\hline $\begin{array}{l}\text { Sulfur-34/Sulfur-32, as sulfate } \\
\text { (low concentration) aqueous }\end{array}$ & & 1949 & -- & -- & 70 \\
\hline $\begin{array}{l}\text { Sulfur-34/Sulfur-32, as sulfide, } \\
\text { aqueous }\end{array}$ & & 1948 & -. & - & 70 \\
\hline $\begin{array}{l}\text { Sulfur-34/Sulfur-32, as sulfate, } \\
\text { rock }\end{array}$ & & 1950 & -- & -- & 70 \\
\hline $\begin{array}{l}\text { Sulfur-34/Sulfur-32, as sulfide, } \\
\text { rock }\end{array}$ & & 1947 & -- & -- & 70 \\
\hline Sulfur-34/Sulfur-32, as sulfate & & 1952 & -- & 921 & 70 \\
\hline Sulfur-34/Sulfur-32, as disulfide & & 1953 & -- & 921 & 70 \\
\hline $\begin{array}{l}\text { Sulfur-34/Sulfur-32, } \\
\text { as monosulfide }\end{array}$ & & 1954 & -- & 921 & 70 \\
\hline
\end{tabular}

